Supporting Information

For

\title{
Hantzsch Ester Mediated Benzannulation of Diazo Compounds under Visible Light Irradiation
}

Savita B. Nagode, ${ }^{\text {a, b }}$ Ruchir Kant, ${ }^{\mathrm{c}}$ Namrata Rastogi*, a, b

${ }^{a}$ Medicinal \& Process Chemistry Division and ${ }^{c}$ Molecular \& Structural Biology Division, CSIRCentral Drug Research Institute, Sector 10, Jankipuram Extension, Sitapur Road, P.O. Box 173, Lucknow 226031, India

${ }^{b}$ Academy of Scientific and Innovative Research, New Delhi 110001, India

namrataiit@gmail.com;namrata.rastogi@cdri.res.in

\section{Table of Contents}

General Information. .2

General Procedures 2-4

Details of CV Experiment. . .4

Characterization Data.

Crystallographic Data for 3zb'. $17-18$

References. .18

Copies of ${ }^{1} \mathrm{H},{ }^{13} \mathrm{C} \&{ }^{31} \mathrm{P}$ NMR Spectra. 19-89 


\section{General Information}

All reactions were monitored by TLC, visualization was effected with UV and/or by developing in iodine. Melting points were recorded on a Precision melting point apparatus and are uncorrected. NMR spectra were recorded on a Brucker Avance spectrometer at $300 / 400 / 500 \mathrm{MHz}\left({ }^{1} \mathrm{H}\right), 75 / 100 / 125 \mathrm{MHz}\left({ }^{13} \mathrm{C}\right)$ and $121.5 / 162 \mathrm{MHz}\left({ }^{31} \mathrm{P}\right)$. Chemical shifts are reported in $\delta(\mathrm{ppm})$ relative to TMS as the internal standard for ${ }^{1} \mathrm{H}$ and ${ }^{13} \mathrm{C}$ and phosphoric acid as the external standard for ${ }^{31} \mathrm{P}$. To describe spin multiplicity, standard abbreviations such as s, d, t, q, m, dd referring to singlet, doublet, triplet, quartet, multiplet and doublet of doublet respectively, are used. The ESI-HRMS spectra were recorded on Agilent 6520- QTofLC/MS system. UV-vis absorption spectroscopy was performed using a Jasco V-750 spectrophotometer, equipped with a peltier unit. Absorption spectra of the samples were recorded in $1 \mathrm{~cm}$ path quartz cuvettes.

The diazo substrates including $\alpha$-diazophosphonates, $\alpha$-diazosulfones, $\alpha$-diazocarboxylates and o-aryl- $\alpha$-diazophosphonates were synthesized from corresponding acylbenotriazoles following reported procedure. ${ }^{1}$ The characterisation data for all the new starting diazo compounds (1f, 1h, 1l-1n and 4a-4i) has been provided in the supporting information. All other chemicals and catalysts were purchased from commercial sources and used as received.

\section{General Procedures}

\section{General procedure for the VLPC benzannulation of $\alpha$-diazo phosphonates/sulfones/carboxylates 1 with alkynes 2}

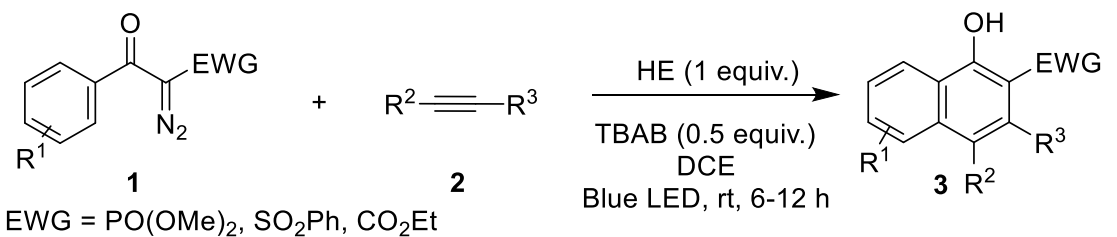

In an oven dried $5 \mathrm{~mL}$ snap vial equipped with a magnetic stirring bar, the $\alpha$-diazo starting compound $1(0.1 \mathrm{mmol})$, alkyne $2(0.2 \mathrm{mmol})$, Hantzsch ester $(25 \mathrm{mg}, 0.1 \mathrm{mmol})$ and TBAB $(16 \mathrm{mg}, 0.05 \mathrm{mmol})$ were dissolved in anhydrous DCE $(1.0 \mathrm{~mL})$. The resulting reaction mixture was degassed by three "pump-freeze-thaw" cycles via a syringe needle. The vial was irradiated using $450 \mathrm{~nm}$ blue LED with a cooling device maintaining the temperature around $25^{\circ} \mathrm{C}$. After $6-12 \mathrm{~h}$ of irradiation (TLC monitoring), the reaction mixture was concentrated under reduced pressure. The residue was purified by column chromatography on silica gel using hexane/ethyl acetate as eluent to afford the pure product $\mathbf{3}$.

\section{Representative example for the synthesis of compound 3a using $1 \mathrm{mmol}$ of compound 1a} In an oven dried $10 \mathrm{~mL}$ snap vial equipped with a magnetic stirring bar, the dimethyl (1diazo-2-oxo-2-phenylethyl)phosphonate 1a $(254 \mathrm{mg}, 1 \mathrm{mmol})$, phenyl acetylene 2a (204 mg, $2 \mathrm{mmol}$ ), Hantzsch ester (253 mg, $1 \mathrm{mmol})$ and TBAB (161 mg, $0.5 \mathrm{mmol}$ ) were dissolved in anhydrous DCE $(6 \mathrm{~mL})$. The resulting reaction mixture was degassed by three "pump-freezethaw" cycles via a syringe needle. The vial was irradiated using $450 \mathrm{~nm}$ blue LED with a cooling device maintaining the temperature around $25^{\circ} \mathrm{C}$. After $6 \mathrm{~h}$ of irradiation (TLC monitoring), the reaction mixture was concentrated under reduced pressure. The residue was 
purified by column chromatography on silica gel using hexane/ethyl acetate as eluent to afford the pure product 3a $(276 \mathrm{mg}, 84 \%)$.

General procedure for the VLPC intramolecular benzannulation of o-aryl $\alpha$-diazo phosphonates 4

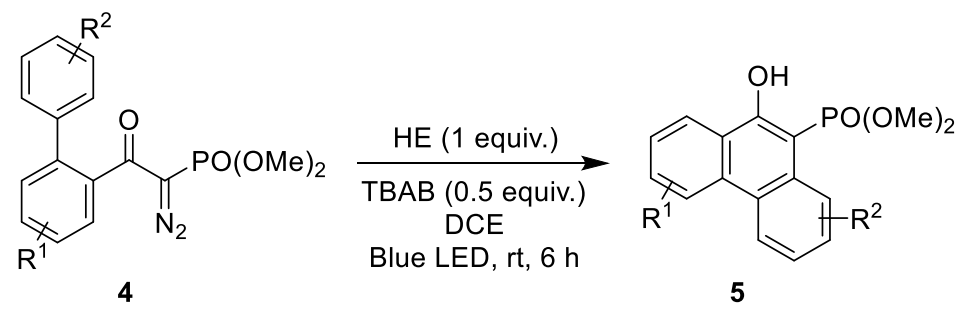

In an oven dried $5 \mathrm{~mL}$ snap vial equipped with a magnetic stirring bar, the o-aryl- $\alpha$ diazophosphonate $4(0.1 \mathrm{mmol})$, Hantzsch ester $(25 \mathrm{mg}, 0.1 \mathrm{mmol})$ and TBAB (16 mg, 0.05 $\mathrm{mmol})$ were dissolved in anhydrous DCE $(1.0 \mathrm{~mL})$. The resulting reaction mixture was degassed by three "pump-freeze-thaw" cycles via a syringe needle. The vial was irradiated using $450 \mathrm{~nm}$ blue LED with a cooling device maintaining the temperature around $25{ }^{\circ} \mathrm{C}$. After $6 \mathrm{~h}$ of irradiation (TLC monitoring), the reaction mixture was concentrated under reduced pressure. The residue was purified by column chromatography on silica gel using hexane/ethyl acetate as eluent to afford the pure product 5 .

\section{Representative example for the synthesis of compound 5 a using $1 \mathrm{mmol}$ of compound 4a}

In an oven dried $10 \mathrm{~mL}$ snap vial equipped with a magnetic stirring bar, the dimethyl (2([1,1'-biphenyl]-2-yl)-1-diazo-2-oxoethyl)phosphonate 4a (330 mg, $1 \mathrm{mmol})$, Hantzsch ester (253 mg, $1 \mathrm{mmol})$ and TBAB (161 mg, $0.5 \mathrm{mmol})$ were dissolved in anhydrous DCE (6 mL). The resulting reaction mixture was degassed by three "pump-freeze-thaw" cycles via a syringe needle. The vial was irradiated using $450 \mathrm{~nm}$ blue LEDs with a cooling device maintaining the temperature around $25{ }^{\circ} \mathrm{C}$. After $6 \mathrm{~h}$ of irradiation (TLC monitoring), the reaction mixture was concentrated under reduced pressure. The residue was purified by column chromatography on silica gel using hexane/ethyl acetate as eluent to afford the pure product 5a (230 mg, 76\%).

\section{Details of radical trapping experiment}

In an oven dried $5 \mathrm{~mL}$ snap vial equipped with a magnetic stirring bar, the dimethyl (1-diazo2-oxo-2-phenylethyl)phosphonate $1 \mathrm{a}(25 \mathrm{mg}, 0.1 \mathrm{mmol})$, was dissolved in anhydrous DCE $(1.0 \mathrm{~mL})$. Further, Hantzsch ester $(25 \mathrm{mg}, 0.1 \mathrm{mmol})$, TBAB (16 mg, $0.05 \mathrm{mmol})$ and allyltributyltin (66 mg, $0.2 \mathrm{mmol}$ ) were added and the resulting reaction mixture was degassed by three "pump-freeze-thaw" cycles via a syringe needle. The vial was irradiated using $450 \mathrm{~nm}$ blue LED with a cooling device maintaining the temperature around $25{ }^{\circ} \mathrm{C}$. After $8 \mathrm{~h}$ of irradiation (TLC monitoring), the reaction mixture was concentrated under reduced pressure. The crude reaction mixture was analyzed by High Resolution Mass Spectrometry (Figure 1). 


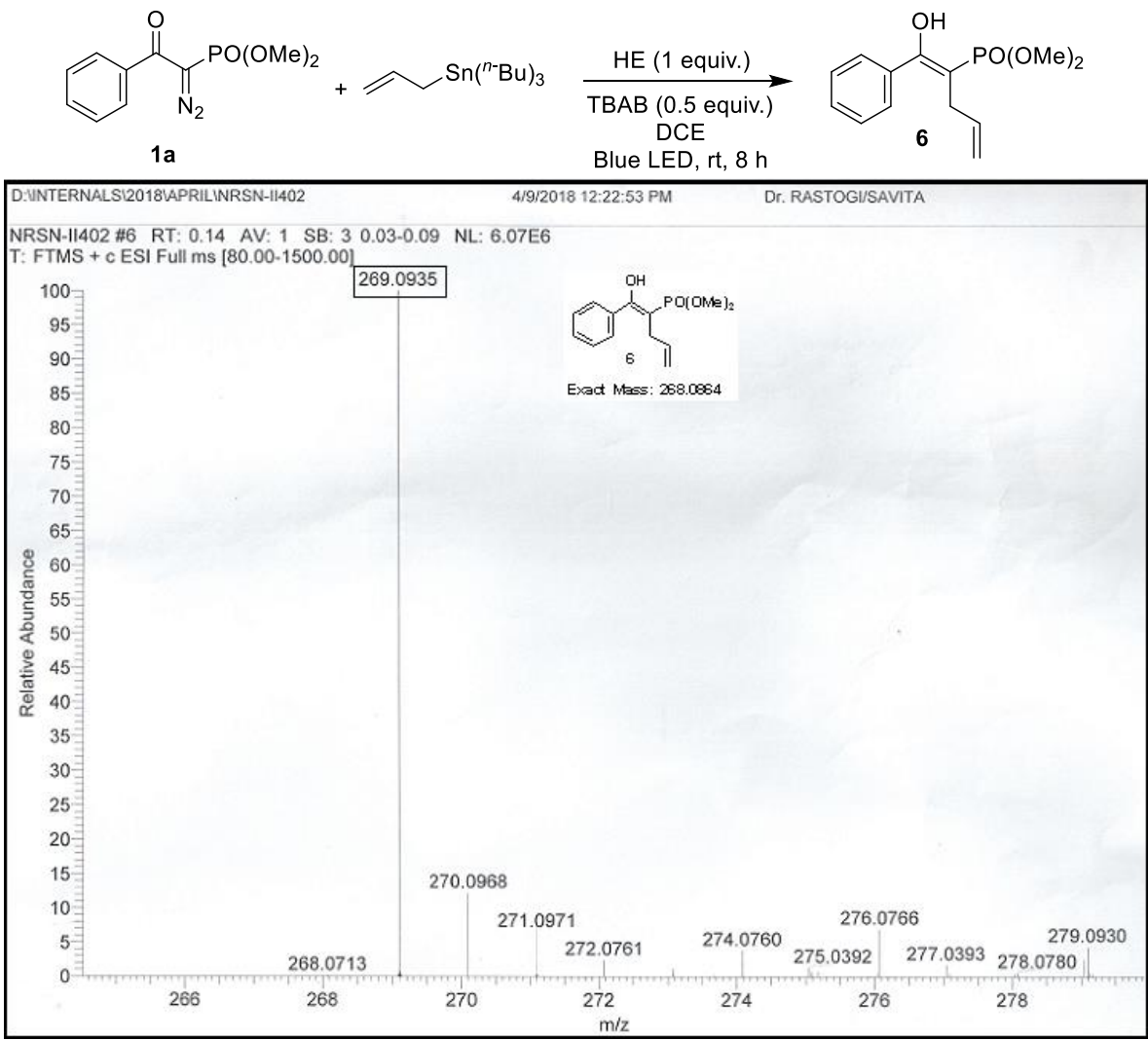

Figure 1. High resolution mass spectrum of 6

\section{Details of Cyclic Voltammentry (CV) Experiment}

Cyclic voltammetry (CV) studies were carried out with an electrochemical system [CH1660E, CH instruments, USA] utilizing the three electrode configuration consisting of glassy carbon (working electrode), platinum wire (auxiliary electrode) and saturated calomel (reference electrode) electrodes. The experiments were carried out under nitrogen atmosphere in dry MeCN using 0.1 M tetrabutylammonium hexafluorophosphonate as the supporting electrolyte. All potentials were calibrated vs. the saturated calomel electrode by the addition of ferrocene as an internal standard, taking $\mathrm{E}_{1 / 2}\left(\mathrm{Fc} / \mathrm{Fc}^{+}\right)=0.38 \mathrm{~V}$ vs. SCE.

The irreversible reduction potential of $\alpha$-diazophosphonate 1a calculated by differential pulse voltammetry (DPV) experiment was Ered $=-1.25 \mathrm{~V}$ vs. SCE in MeCN (Figure 2).

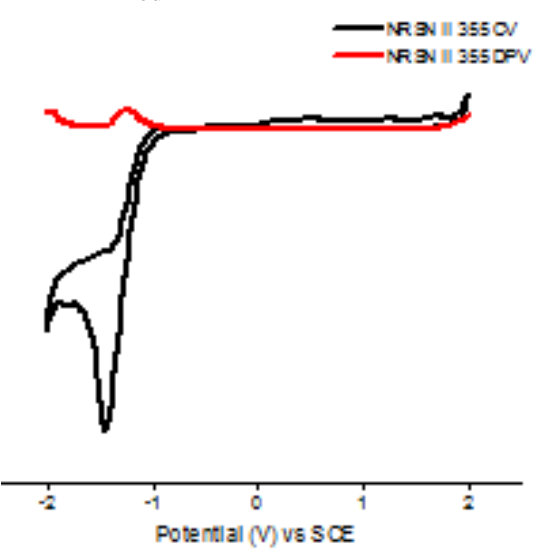

Figure 2. Cyclic voltammograms and differential pulse voltammograms of NRSN II 355 (1a) recorded in $\mathrm{CH}_{3} \mathrm{CN}$ with $0.1 \mathrm{M} \mathrm{n}-\mathrm{Bu}_{4} \mathrm{NPF}_{6}$ as the supporting electrolyte and scan rate is 0.05 $\mathrm{Vs}^{-1}$. 


\section{Characterization Data}

\subsection{Characterization data for starting materials}

Dimethyl (1-diazo-2-(naphthalen-2-yl)-2-oxoethyl)phosphonate (1f)

Yellow viscous liquid; isolated yield $84 \%(255 \mathrm{mg}) . R_{f} 0.50\left(40 \%\right.$ EtOAc/hexane); ${ }^{1} \mathrm{H}$ NMR

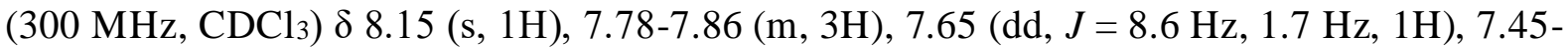
$7.55(\mathrm{~m}, 2 \mathrm{H}), 3.76\left(\mathrm{~d},{ }^{3} J_{\mathrm{H}-\mathrm{P}}=11.9 \mathrm{~Hz}, 6 \mathrm{H}\right) ;{ }^{13} \mathrm{C} \mathrm{NMR}\left(75 \mathrm{MHz}, \mathrm{CDCl}_{3}\right) \delta 187.36\left(\mathrm{~d},{ }^{2} J_{\mathrm{C}-\mathrm{P}}=\right.$ $9.3 \mathrm{~Hz}), 135.26,134.15\left(\mathrm{~d}, J_{\mathrm{C}-\mathrm{P}}=2.7 \mathrm{~Hz}\right), 132.39,129.32,128.85,128.55,128.53,128.03$, $127.25,123.76,63.36\left(\mathrm{~d},{ }^{1} J_{\mathrm{C}-\mathrm{P}}=217.4 \mathrm{~Hz}\right), 54.22\left(\mathrm{~d},{ }^{2} J_{\mathrm{C}-\mathrm{P}}=6.0 \mathrm{~Hz}\right) ;{ }^{31} \mathrm{P}$ NMR $(121.5 \mathrm{MHz}$, $\left.\mathrm{CDCl}_{3}\right) \delta$ 13.91; HRMS for $\mathrm{C}_{14} \mathrm{H}_{13} \mathrm{~N}_{2} \mathrm{O} 4 \mathrm{P}$ : calcd. $\left(\mathrm{MH}^{+}\right)$: 305.0686, found: 305.0687

\section{Dimethyl (2-(benzofuran-2-yl)-1-diazo-2-oxoethyl)phosphonate (1h)}

Yellow viscous liquid; isolated yield $82 \%(241 \mathrm{mg}) . R_{f} 0.50\left(40 \%\right.$ EtOAc/hexane); ${ }^{1} \mathrm{H}$ NMR $\left(300 \mathrm{MHz}, \mathrm{CDCl}_{3}\right) \delta 7.63(\mathrm{~d}, J=7.8 \mathrm{~Hz}, 1 \mathrm{H}), 7.50(\mathrm{~s}, 1 \mathrm{H}), 7.38-7.47(\mathrm{~m}, 2 \mathrm{H}), 7.23-7.28(\mathrm{~m}$, $1 \mathrm{H}), 3.83\left(\mathrm{~d},{ }^{3} J_{\mathrm{H}-\mathrm{P}}=12.1 \mathrm{~Hz}, 6 \mathrm{H}\right) ;{ }^{13} \mathrm{C}$ NMR $\left(100 \mathrm{MHz}, \mathrm{CDCl}_{3}\right) \delta 173.75\left(\mathrm{~d},{ }^{2} J_{\mathrm{C}-\mathrm{P}}=8.2 \mathrm{~Hz}\right)$, $154.79,151.47\left(\mathrm{~d}, J_{\mathrm{C}-\mathrm{P}}=5.0 \mathrm{~Hz}\right), 128.22,126.54,124.17,123.15,112.84,112.15,61.99(\mathrm{~d}$, $\left.{ }^{1} J_{\mathrm{C}-\mathrm{P}}=218.0 \mathrm{~Hz}\right), 54.15\left(\mathrm{~d},{ }^{2} J_{\mathrm{C}-\mathrm{P}}=5.8 \mathrm{~Hz}\right) ;{ }^{31} \mathrm{P} \mathrm{NMR}\left(121.5 \mathrm{MHz}, \mathrm{CDCl}_{3}\right) \delta 13.73$; HRMS for $\mathrm{C}_{12} \mathrm{H}_{11} \mathrm{~N}_{2} \mathrm{O} 5 \mathrm{P}$ : calcd. $\left(\mathrm{MH}^{+}\right)$: 295.0478, found: 295.0477

\section{Dimethyl (1-diazo-2-(5-methylthiophen-2-yl)-2-oxoethyl)phosphonate (11)}

Yellow viscous liquid; isolated yield $68 \%(186 \mathrm{mg}) . R_{f} 0.50$ (40\% EtOAc/hexane); ${ }^{1} \mathrm{H}$ NMR $\left(300 \mathrm{MHz} \mathrm{CDCl}_{3}\right) \delta 7.50(\mathrm{~d}, J=3.8 \mathrm{~Hz}, 1 \mathrm{H}), 6.71(\mathrm{dd}, J=3.9 \mathrm{~Hz}, 1.0 \mathrm{~Hz}, 1 \mathrm{H}), 3.78\left(\mathrm{~d},{ }^{3} J_{\mathrm{H}-\mathrm{P}}\right.$ $=12.0 \mathrm{~Hz}, 6 \mathrm{H}), 2.45(\mathrm{~s}, 1 \mathrm{H}) ;{ }^{13} \mathrm{C} \mathrm{NMR}\left(75 \mathrm{MHz}, \mathrm{CDCl}_{3}\right) \delta 176.47\left(\mathrm{~d},{ }^{2} J_{\mathrm{C}-\mathrm{P}}=9.7 \mathrm{~Hz}\right)$, $149.39,139.02\left(\mathrm{~d}, J_{\mathrm{C}-\mathrm{P}}=2.7 \mathrm{~Hz}\right), 131.66,126.58,61.69\left(\mathrm{~d},{ }^{1} J_{\mathrm{C}-\mathrm{P}}=215.9 \mathrm{~Hz}\right), 53.83\left(\mathrm{~d},{ }^{2} J_{\mathrm{C}-\mathrm{P}}\right.$ $=5.7 \mathrm{~Hz}), 15.58 ;{ }^{31} \mathrm{P}$ NMR $\left(121.5 \mathrm{MHz}, \mathrm{CDCl}_{3}\right) \delta$ 13.98; HRMS for $\mathrm{C}_{9} \mathrm{H}_{11} \mathrm{~N}_{2} \mathrm{O}_{4} \mathrm{PS}$ : calcd. $\left(\mathrm{MH}^{+}\right): 275.0250$, found: 275.0251

\section{Dimethyl (1-diazo-2-(furan-3-yl)-2-oxoethyl)phosphonate (1m)}

Yellow viscous liquid; isolated yield $81 \%(198 \mathrm{mg}) . R_{f} 0.50$ (40\% EtOAc/hexane); ${ }^{1} \mathrm{H}$ NMR $\left(300 \mathrm{MHz}, \mathrm{CDCl}_{3}\right) \delta 8.04(\mathrm{~s}, 1 \mathrm{H}), 7.38(\mathrm{~s}, 1 \mathrm{H}), 6.74(\mathrm{~s}, 1 \mathrm{H}), 3.78\left(\mathrm{~d},{ }^{3} J_{\mathrm{H}-\mathrm{P}}=11.9 \mathrm{~Hz}, 6 \mathrm{H}\right)$; ${ }^{13} \mathrm{C}$ NMR $\left(75 \mathrm{MHz}, \mathrm{CDCl}_{3}\right) \delta 178.69\left(\mathrm{~d},{ }^{2} J_{\mathrm{C}-\mathrm{P}}=10.5 \mathrm{~Hz}\right), 146.63,143.78,125.19,109.49$, $63.92\left(\mathrm{~d},{ }^{1} J_{\mathrm{C}-\mathrm{P}}=215.8 \mathrm{~Hz}\right), 54.10\left(\mathrm{~d},{ }^{2} J_{\mathrm{C}-\mathrm{P}}=5.6 \mathrm{~Hz}\right) ;{ }^{31} \mathrm{P}$ NMR $\left(121.5 \mathrm{MHz}, \mathrm{CDCl}_{3}\right) \delta 13.69$; HRMS for $\mathrm{C}_{8} \mathrm{H}_{9} \mathrm{~N}_{2} \mathrm{O} \mathrm{O}_{5} \mathrm{P}$ : calcd. $\left(\mathrm{MH}^{+}\right)$: 245.0322, found: 245.0319

\section{Dimethyl (1-diazo-2-oxo-2-(thiophen-3-yl)ethyl)phosphonate (1n)}

Yellow viscous liquid; isolated yield $61 \%(159 \mathrm{mg}) . R_{f} 0.50$ (40\% EtOAc/hexane); ${ }^{1} \mathrm{H}$ NMR $\left(300 \mathrm{MHz}, \mathrm{CDCl}_{3}\right) \delta 8.00(\mathrm{dd}, J=2.9 \mathrm{~Hz}, 1.3 \mathrm{~Hz}, 1 \mathrm{H}), 7.41(\mathrm{dd}, J=5.1 \mathrm{~Hz}, 1.3 \mathrm{~Hz}, 1 \mathrm{H})$, $7.28(\mathrm{dd}, J=5.1 \mathrm{~Hz}, 2.9 \mathrm{~Hz}, 1 \mathrm{H}), 3.78\left(\mathrm{~d},{ }^{3} J_{\mathrm{H}-\mathrm{P}}=12.0 \mathrm{~Hz}, 6 \mathrm{H}\right) ;{ }^{13} \mathrm{C} \mathrm{NMR}\left(75 \mathrm{MHz}, \mathrm{CDCl}_{3}\right)$ $\delta 179.93\left(\mathrm{~d},{ }^{2} J_{\mathrm{C}-\mathrm{P}}=9.9 \mathrm{~Hz}\right), 139.17\left(\mathrm{~d}, J_{\mathrm{C}-\mathrm{P}}=1.8 \mathrm{~Hz}\right), 130.87,127.08,126.33,63.31\left(\mathrm{~d},{ }^{1} J_{\mathrm{C}-\mathrm{P}}\right.$ $=219.0 \mathrm{~Hz}), 54.01\left(\mathrm{~d},{ }^{2} J_{\mathrm{C}-\mathrm{P}}=5.9 \mathrm{~Hz}\right) ;{ }^{31} \mathrm{P} \mathrm{NMR}\left(121.5 \mathrm{MHz}, \mathrm{CDCl}_{3}\right) \delta 13.94$; HRMS for $\mathrm{C}_{8} \mathrm{H}_{9} \mathrm{~N}_{2} \mathrm{O}_{4} \mathrm{PS}$ : calcd. $\left(\mathrm{MH}^{+}\right)$: 261.0093, found: 261.0095

\section{Dimethyl (2-([1,1'-biphenyl]-2-yl)-1-diazo-2-oxoethyl)phosphonate (4a)}

Yellow viscous liquid; isolated yield $72 \%(238 \mathrm{mg}) . R_{f} 0.50\left(40 \%\right.$ EtOAc/hexane); ${ }^{1} \mathrm{H}$ NMR $\left(300 \mathrm{MHz}, \mathrm{CDCl}_{3}\right) \delta$ 7.44-7.49 (m, $\left.1 \mathrm{H}\right), 7.32-7.39(\mathrm{~m}, 8 \mathrm{H}), 3.58\left(\mathrm{~d},{ }^{3} \mathrm{~J}_{\mathrm{H}-\mathrm{P}}=12.0 \mathrm{~Hz}, 6 \mathrm{H}\right) ;{ }^{13} \mathrm{C}$ NMR $\left(75 \mathrm{MHz}, \mathrm{CDCl}_{3}\right) \delta 190.01\left(\mathrm{~d},{ }^{2} J_{\mathrm{C}-\mathrm{P}}=7.4 \mathrm{~Hz}\right), 139.35,139.00,136.79\left(\mathrm{~d}, J_{\mathrm{C}-\mathrm{P}}=5.1\right.$ $\mathrm{Hz}), 131.02,130.02,128.99,128.64,128.27,127.93,127.44,53.90\left(\mathrm{~d},{ }^{2} J_{\mathrm{C}-\mathrm{P}}=5.8 \mathrm{~Hz}\right) ;{ }^{31} \mathrm{P}$ NMR (121.5 MHz, $\left.\mathrm{CDCl}_{3}\right) \delta$ 12.77; HRMS for $\mathrm{C}_{16} \mathrm{H}_{15} \mathrm{~N}_{2} \mathrm{O} 4 \mathrm{P}$ : calcd. $\left(\mathrm{MH}^{+}\right)$: 331.0842, found: 331.0843 
Dimethyl (1-diazo-2-(4'-methyl-[1,1'-biphenyl]-2-yl)-2-oxoethyl)phosphonate (4b)

Yellow viscous liquid; isolated yield $78 \%(268 \mathrm{mg}) . R_{f} 0.50$ (40\% EtOAc/hexane); ${ }^{1} \mathrm{H}$ NMR $\left(400 \mathrm{MHz}, \mathrm{CDCl}_{3}\right) \delta$ 7.42-7.46 (m, 1H), 7.30-7.39 (m, 3H), 7.25-7.28 (m, 2H), 7.13-7.15 (m appearing as d, 2H), $3.60\left(\mathrm{~d},{ }^{3} J_{\mathrm{H}-\mathrm{P}}=12.0 \mathrm{~Hz}, 6 \mathrm{H}\right), 2.30(\mathrm{~s}, 3 \mathrm{H}) ;{ }^{13} \mathrm{C} \mathrm{NMR}\left(125 \mathrm{MHz}, \mathrm{CDCl}_{3}\right)$ $\delta 190.15\left(\mathrm{~d},{ }^{2} J_{\mathrm{C}-\mathrm{P}}=7.2 \mathrm{~Hz}\right), 139.38,138.19,136.71\left(\mathrm{~d}, J_{\mathrm{C}-\mathrm{P}}=4.8 \mathrm{~Hz}\right), 136.06,131.00$, 129.96, 129.34, 128.87, 127.66, 127.50, $64.56\left(\mathrm{~d},{ }^{1} J_{\mathrm{C}-\mathrm{P}}=214.7 \mathrm{~Hz}\right), 53.90\left(\mathrm{~d},{ }^{2} J_{\mathrm{C}-\mathrm{P}}=5.6 \mathrm{~Hz}\right)$, 21.15; ${ }^{31} \mathrm{P}$ NMR $\left(121.5 \mathrm{MHz}, \mathrm{CDCl}_{3}\right) \delta$ 13.05; HRMS for $\mathrm{C}_{17} \mathrm{H}_{17} \mathrm{~N}_{2} \mathrm{O}_{4} \mathrm{P}$ : calcd. $\left(\mathrm{MH}^{+}\right)$: 345.0999, found: 345.1001

\section{Dimethyl (1-diazo-2-(4'-methoxy-[1,1'-biphenyl]-2-yl)-2-oxoethyl)phosphonate (4c)}

Yellow viscous liquid; isolated yield $80 \%(288 \mathrm{mg}) . R_{f} 0.50$ (40\% EtOAc/hexane); ${ }^{1} \mathrm{H}$ NMR $\left(300 \mathrm{MHz}, \mathrm{CDCl}_{3}\right) \delta$ 7.40-7.44 (m, 1H), 7.26-7.39 (m, 5H), 6.82-6.87 (m, 2H), $3.73(\mathrm{~s}, 3 \mathrm{H})$, $3.60\left(\mathrm{~d},{ }^{3} J_{\mathrm{H}-\mathrm{P}}=12.0 \mathrm{~Hz}, 6 \mathrm{H}\right) ;{ }^{13} \mathrm{C} \mathrm{NMR}\left(75 \mathrm{MHz}, \mathrm{CDCl}_{3}\right) \delta 190.36\left(\mathrm{~d},{ }^{2} J_{\mathrm{C}-\mathrm{P}}=7.5 \mathrm{~Hz}\right)$, $159.91,139.12,136.73\left(\mathrm{~d}, J_{\mathrm{C}-\mathrm{P}}=5.2 \mathrm{~Hz}\right), 131.36,131.12,130.26,129.98,127.64,127.57$, $114.21,64.61\left(\mathrm{~d},{ }^{1} J_{\mathrm{C}-\mathrm{P}}=215.9 \mathrm{~Hz}\right), 55.43,54.03\left(\mathrm{~d},{ }^{2} J_{\mathrm{C}-\mathrm{P}}=5.8 \mathrm{~Hz}\right) ;{ }^{31} \mathrm{P}$ NMR $(121.5 \mathrm{MHz}$, $\left.\mathrm{CDCl}_{3}\right) \delta$ 13.02; HRMS for $\mathrm{C}_{17} \mathrm{H}_{17} \mathrm{~N}_{2} \mathrm{O} 5 \mathrm{P}$ : calcd. $\left(\mathrm{MH}^{+}\right)$: 361.0948 , found: 361.0947

\section{Dimethyl (2-(4' -chloro-[1,1'-biphenyl]-2-yl)-1-diazo-2-oxoethyl)phosphonate (4d)}

Yellow viscous liquid; isolated yield $76 \%(277 \mathrm{mg}) . R_{f} 0.50$ (40\% EtOAc/hexane); ${ }^{1} \mathrm{H}$ NMR $\left(400 \mathrm{MHz}, \mathrm{CDCl}_{3}\right) \delta 7.45-7.49(\mathrm{~m}, 1 \mathrm{H}), 7.39(\mathrm{~m}$ appearing as $\mathrm{d}, 2 \mathrm{H}), 3.62\left(\mathrm{~d},{ }^{3} J_{\mathrm{H}-\mathrm{P}}=12.0\right.$ $\mathrm{Hz}, 6 \mathrm{H}) ;{ }^{13} \mathrm{C} \mathrm{NMR}\left(100 \mathrm{MHz}, \mathrm{CDCl}_{3}\right) \delta 189.73\left(\mathrm{~d},{ }^{2} J_{\mathrm{C}-\mathrm{P}}=7.9 \mathrm{~Hz}\right), 138.14,137.41,136.68$ $\left(\mathrm{d}, J_{\mathrm{C}-\mathrm{P}}=4.6 \mathrm{~Hz}\right), 134.56,131.15,130.25,129.92,128.82,128.20,127.50,53.97\left(\mathrm{~d},{ }^{2} J_{\mathrm{C}-\mathrm{P}}=\right.$ $5.7 \mathrm{~Hz}) ;{ }^{31} \mathrm{P}$ NMR $\left(121.5 \mathrm{MHz}, \mathrm{CDCl}_{3}\right) \delta$ 12.53; HRMS for $\mathrm{C}_{16} \mathrm{H}_{14} \mathrm{ClN}_{2} \mathrm{O} 4 \mathrm{P}$ : calcd. $\left(\mathrm{MH}^{+}\right)$: 365.0452, found: 365.0444

\section{Dimethyl (1-diazo-2-(4'-fluoro-[1,1'-biphenyl]-2-yl)-2-oxoethyl)phosphonate (4e)}

Yellow viscous liquid; isolated yield $70 \%$ (244 mg). $R_{f} 0.50$ (40\% EtOAc/hexane); ${ }^{1} \mathrm{H}$ NMR $\left(300 \mathrm{MHz} \mathrm{CDCl}_{3}\right) \delta$ 7.44-7.49 (m, 1H), 7.29-7.40 (m, 5H), 7.00-7.08 (m, 2H), $3.62\left(\mathrm{~d},{ }^{3} J_{\mathrm{H}-\mathrm{P}}\right.$ $=12.0 \mathrm{~Hz}, 6 \mathrm{H}) ;{ }^{13} \mathrm{C} \mathrm{NMR}\left(75 \mathrm{MHz}, \mathrm{CDCl}_{3}\right) \delta 189.84\left(\mathrm{~d},{ }^{2} J_{\mathrm{C}-\mathrm{P}}=7.9 \mathrm{~Hz}\right), 162.86\left(\mathrm{~d},{ }^{1} J_{\mathrm{C}-\mathrm{F}}=\right.$ $246.6 \mathrm{~Hz}), 138.29,136.77\left(\mathrm{~d}, J_{\mathrm{C}-\mathrm{P}}=4.8 \mathrm{~Hz}\right), 135.01\left(\mathrm{~d}, J_{\mathrm{C}-\mathrm{F}}=3.2 \mathrm{~Hz}\right), 131.07,130.68\left(\mathrm{~d}, J_{\mathrm{C}-\mathrm{F}}\right.$ $=8.1 \mathrm{~Hz}), 130.01,127.98,127.45,115.62\left(\mathrm{~d}, J_{\mathrm{C}-\mathrm{F}}=21.5 \mathrm{~Hz}\right), 53.92\left(\mathrm{~d},{ }^{2} J_{\mathrm{C}-\mathrm{P}}=6.0 \mathrm{~Hz}\right) ;{ }^{31} \mathrm{P}$ NMR $\left(121.5 \mathrm{MHz}, \mathrm{CDCl}_{3}\right) \delta$ 12.56; HRMS for $\mathrm{C}_{16} \mathrm{H}_{14} \mathrm{FN}_{2} \mathrm{O}_{4} \mathrm{P}$ : calcd. $\left(\mathrm{MH}^{+}\right)$: 349.0748, found: 349.0745

\section{Dimethyl (1-diazo-2-oxo-2-(4'-(trifluoromethyl)-[1,1'-biphenyl]-2-yl)ethyl)phosphonate (4f)}

Yellow viscous liquid; isolated yield $68 \%(271 \mathrm{mg}) . R_{f} 0.50$ (40\% EtOAc/hexane); ${ }^{1} \mathrm{H}$ NMR $\left(300 \mathrm{MHz}, \mathrm{CDCl}_{3}\right) \delta$ 7.60-7.63 (m, 2H), 7.46-7.53 (m, 3H), 7.41-7.44 (m, 2H), 7.32-7.35 (m, $1 \mathrm{H}), 3.57\left(\mathrm{~d},{ }^{3} J_{\mathrm{H}-\mathrm{P}}=11.9 \mathrm{~Hz}, 6 \mathrm{H}\right) ;{ }^{13} \mathrm{C} \mathrm{NMR}\left(75 \mathrm{MHz}, \mathrm{CDCl}_{3}\right) \delta 189.53\left(\mathrm{~d},{ }^{2} J_{\mathrm{C}-\mathrm{P}}=8.2 \mathrm{~Hz}\right)$, $142.90,138.18,137.07\left(\mathrm{~d}, J_{\mathrm{C}-\mathrm{P}}=4.2 \mathrm{~Hz}\right), 131.34,130.52\left(\mathrm{q}, J_{\mathrm{C}-\mathrm{F}}=32.4 \mathrm{~Hz}\right), 130.19,129.55$, 128.76, 127.60, $125.67\left(\mathrm{q}, J_{\mathrm{C}-\mathrm{F}}=3.7 \mathrm{~Hz}\right), 124.17\left(\mathrm{q},{ }^{1} J_{\mathrm{C}-\mathrm{F}}=270.4 \mathrm{~Hz}\right), 65.15\left(\mathrm{~d},{ }^{1} J_{\mathrm{C}-\mathrm{P}}=215.3\right.$ $\mathrm{Hz}), 54.03\left(\mathrm{~d},{ }^{2} J_{\mathrm{C}-\mathrm{P}}=5.9 \mathrm{~Hz}\right) ;{ }^{31} \mathrm{P} \mathrm{NMR}\left(121.5 \mathrm{MHz}, \mathrm{CDCl}_{3}\right) \delta 12.19$; HRMS for $\mathrm{C}_{17} \mathrm{H}_{14} \mathrm{~F}_{3} \mathrm{~N}_{2} \mathrm{O} 4 \mathrm{P}$ : calcd. $\left(\mathrm{MH}^{+}\right)$: 399.0716, found: 399.0717

\section{Dimethyl (1-diazo-2-(2-(naphthalen-2-yl)phenyl)-2-oxoethyl)phosphonate (4g)}

Yellow viscous liquid; isolated yield $74 \%(281 \mathrm{mg}) . R_{f} 0.50\left(40 \%\right.$ EtOAc/hexane); ${ }^{1} \mathrm{H}$ NMR $\left(300 \mathrm{MHz}, \mathrm{CDCl}_{3}\right) \delta$ 7.77-7.84 (m, 4H), 7.49-7.54 (m, 2H), 7.41-7.46 (m, 5H), $3.43\left(\mathrm{~d},{ }^{3} J_{\mathrm{H}-\mathrm{P}}\right.$ $=12.0 \mathrm{~Hz}, 6 \mathrm{H}) ;{ }^{13} \mathrm{C} \mathrm{NMR}\left(75 \mathrm{MHz}, \mathrm{CDCl}_{3}\right) \delta 189.99\left(\mathrm{~d},{ }^{2} J_{\mathrm{C}-\mathrm{P}}=7.4 \mathrm{~Hz}\right), 139.30,136.98(\mathrm{~d}$, $\left.J_{\mathrm{C}-\mathrm{P}}=5.1 \mathrm{~Hz}\right), 136.24,133.12,132.81,131.04,130.24,128.40,128.28,128.15,127.95$, 
$127.67,127.56,126.65,126.62,53.71\left(\mathrm{~d},{ }^{2} J_{\mathrm{C}-\mathrm{P}}=5.8 \mathrm{~Hz}\right) ;{ }^{31} \mathrm{P} \mathrm{NMR}\left(162 \mathrm{MHz}, \mathrm{CDCl}_{3}\right) \delta$ 12.77; HRMS for $\mathrm{C}_{20} \mathrm{H}_{17} \mathrm{~N}_{2} \mathrm{O}_{4} \mathrm{P}$ : calcd. $\left(\mathrm{MH}^{+}\right)$: 381.0999 , found: 381.0997

Dimethyl (1-diazo-2-(3',4'-dimethoxy-[1,1'-biphenyl]-2-yl)-2-oxoethyl)phosphonate (4h) Yellow viscous liquid; isolated yield $74 \%(289 \mathrm{mg}) . R_{f} 0.50\left(40 \%\right.$ EtOAc/hexane); ${ }^{1} \mathrm{H}$ NMR $\left(400 \mathrm{MHz}, \mathrm{CDCl}_{3}\right) \delta$ 7.43-7.48 (m, 1H), 7.33-7.37 (m, 3H), 6.93-6.97 (m, 1H), 6.84-6.88 (m, $2 \mathrm{H}), 3.83(\mathrm{~s}, 3 \mathrm{H}), 3.81(\mathrm{~s}, 3 \mathrm{H}), 3.61\left(\mathrm{~d},{ }^{3} J_{\mathrm{H}-\mathrm{P}}=12.0 \mathrm{~Hz}, 6 \mathrm{H}\right) ;{ }^{13} \mathrm{C} \mathrm{NMR}\left(125 \mathrm{MHz}, \mathrm{CDCl}_{3}\right) \delta$ $190.36\left(\mathrm{~d},{ }^{2} J_{\mathrm{C}-\mathrm{P}}=7.3 \mathrm{~Hz}\right), 149.24,148.81,139.20,136.74\left(\mathrm{~d}, J_{\mathrm{C}-\mathrm{P}}=4.9 \mathrm{~Hz}\right), 131.59,130.98$, $129.77,127.59,127.42,121.19,112.48,111.31,64.53\left(\mathrm{~d},{ }^{1} J_{\mathrm{C}-\mathrm{P}}=216.8 \mathrm{~Hz}\right), 55.96,55.87$, $53.79\left(\mathrm{~d},{ }^{2} J_{\mathrm{C}-\mathrm{P}}=5.6 \mathrm{~Hz}\right)$; ${ }^{31} \mathrm{P}$ NMR $\left(162 \mathrm{MHz}, \mathrm{CDCl}_{3}\right) \delta 13.17$; HRMS for $\mathrm{C}_{18} \mathrm{H}_{19} \mathrm{~N}_{2} \mathrm{O} 6 \mathrm{P}$ : calcd. $\left(\mathrm{MH}^{+}\right)$: 391.1053, found: 391.1052

\section{Dimethyl (1-diazo-2-oxo-2-(5-(trifluoromethyl)-[1,1'-biphenyl]-2-yl)ethyl)phosphonate} (4i)

Yellow viscous liquid; isolated yield $66 \%$ (263 mg). $R_{f} 0.50$ (40\% EtOAc/hexane); ${ }^{1} \mathrm{H}$ NMR $\left(300 \mathrm{MHz}, \mathrm{CDCl}_{3}\right) \delta$ 7.58-7.61 (m, 2H), 7.44-7.51 (m, 3H), $7.41(\mathrm{~d}, J=3.8 \mathrm{~Hz}, 2 \mathrm{H}), 7.32(\mathrm{~d}$, $J=7.4 \mathrm{~Hz}, 1 \mathrm{H}), 3.55\left(\mathrm{~d},{ }^{3} J_{\mathrm{H}-\mathrm{P}}=11.9 \mathrm{~Hz}, 6 \mathrm{H}\right) ;{ }^{13} \mathrm{C} \mathrm{NMR}\left(75 \mathrm{MHz}, \mathrm{CDCl}_{3}\right) \delta 189.51\left(\mathrm{~d},{ }^{2} J_{\mathrm{C}-\mathrm{P}}\right.$ $=8.0 \mathrm{~Hz}), 142.87,138.15,137.01\left(\mathrm{~d}, J_{\mathrm{C}-\mathrm{P}}=4.2 \mathrm{~Hz}\right), 131.34,130.48\left(\mathrm{q}, J_{\mathrm{C}-\mathrm{F}}=32.6 \mathrm{~Hz}\right)$, $130.17,129.53,128.74,127.58,125.64\left(\mathrm{q}, J_{\mathrm{C}-\mathrm{F}}=3.6 \mathrm{~Hz}\right), 124.15\left(\mathrm{q},{ }^{1} J_{\mathrm{C}-\mathrm{F}}=270.5 \mathrm{~Hz}\right), 65.09$ $\left(\mathrm{d},{ }^{1} J_{\mathrm{C}-\mathrm{P}}=216.9 \mathrm{~Hz}\right), 54.04\left(\mathrm{~d},{ }^{2} J_{\mathrm{C}-\mathrm{P}}=5.9 \mathrm{~Hz}\right) ;{ }^{31} \mathrm{P} \mathrm{NMR}\left(121.5 \mathrm{MHz}, \mathrm{CDCl}_{3}\right) \delta 12.21$; HRMS for $\mathrm{C}_{17} \mathrm{H}_{14} \mathrm{~F}_{3} \mathrm{~N}_{2} \mathrm{O}_{4} \mathrm{P}$ : calcd. $\left(\mathrm{MH}^{+}\right)$: 399.0716 , found: 399.0719

\subsection{Characterization data for products}

Dimethyl (1-hydroxy-4-phenylnaphthalen-2-yl)phosphonate (3a) ${ }^{2}$

White solid; isolated yield 84\% (28 mg). $R_{f} 0.50$ (10\% EtOAc/hexane); Mp $102-103{ }^{\circ} \mathrm{C} ;{ }^{1} \mathrm{H}$ NMR $\left(400 \mathrm{MHz}, \mathrm{CDCl}_{3}\right) \delta 11.07(\mathrm{~s}, 1 \mathrm{H}), 8.39-8.42(\mathrm{~m}, 1 \mathrm{H}), 7.76-7.78(\mathrm{~m}, 1 \mathrm{H}), 7.47-7.50$ $(\mathrm{m}, 2 \mathrm{H}), 7.32-7.42(\mathrm{~m}, 5 \mathrm{H}), 7.14\left(\mathrm{~d},{ }^{3} J_{\mathrm{H}-\mathrm{P}}=12.9 \mathrm{~Hz}, 1 \mathrm{H}\right), 3.72\left(\mathrm{~d},{ }^{3} J_{\mathrm{H}-\mathrm{P}}=11.5 \mathrm{~Hz}, 6 \mathrm{H}\right) ;{ }^{13} \mathrm{C}$ NMR $\left(100 \mathrm{MHz}, \mathrm{CDCl}_{3}\right) \delta 160.93\left(\mathrm{~d},{ }^{2} J_{\mathrm{C}-\mathrm{P}}=7.0 \mathrm{~Hz}, \mathrm{C}-\mathrm{OH}\right), 139.88,135.40,132.14\left(\mathrm{~d}, J_{\mathrm{C}-\mathrm{P}}\right.$ $=13.3 \mathrm{~Hz}), 130.17,129.34,128.39,127.33,125.92\left(\mathrm{~d}, J_{\mathrm{C}-\mathrm{P}}=6.0 \mathrm{~Hz}\right), 125.79\left(\mathrm{~d}, J_{\mathrm{C}-\mathrm{P}}=6.6\right.$ $\mathrm{Hz}), 125.01\left(\mathrm{~d}, J_{\mathrm{C}-\mathrm{P}}=13.7 \mathrm{~Hz}\right), 123.91,98.70\left(\mathrm{~d},{ }^{1} J_{\mathrm{C}-\mathrm{P}}=181.9 \mathrm{~Hz}\right), 53.03\left(\mathrm{~d},{ }^{2} J_{\mathrm{C}-\mathrm{P}}=4.5 \mathrm{~Hz}\right.$, $\left.\{\mathrm{PO}\} \mathrm{OCH}_{3} \times 2\right) ;{ }^{31} \mathrm{P} \mathrm{NMR}\left(162 \mathrm{MHz}, \mathrm{CDCl}_{3}\right) \delta 26.79$; HRMS for $\mathrm{C}_{18} \mathrm{H}_{17} \mathrm{O}_{4} \mathrm{P}:$ calcd. $\left(\mathrm{MH}^{+}\right)$: 329.0937, found: 329.0942

\section{Dimethyl (1-hydroxy-6-methoxy-4-phenylnaphthalen-2-yl)phosphonate (3b)}

White solid; isolated yield $81 \%$ (29 mg). $R_{f} 0.50$ (10\% EtOAc/hexane); Mp 114-116 ${ }^{\circ} \mathrm{C} ;{ }^{1} \mathrm{H}$ NMR $\left(500 \mathrm{MHz}, \mathrm{CDCl}_{3}\right) \delta 11.09(\mathrm{~s}, 1 \mathrm{H}), 8.37(\mathrm{~d}, J=9.9 \mathrm{~Hz}, 1 \mathrm{H}), 7.46-7.48(\mathrm{~m}, 4 \mathrm{H}), 7.37-$ $7.42(\mathrm{~m}, 1 \mathrm{H}), 7.16-7.19(\mathrm{~m}, 3 \mathrm{H}), 3.77\left(\mathrm{~d},{ }^{3} \mathrm{~J}_{\mathrm{H}-\mathrm{P}}=11.5 \mathrm{~Hz}, 6 \mathrm{H}\right), 3.75(\mathrm{~s}, 3 \mathrm{H}) ;{ }^{13} \mathrm{C}$ NMR $(100$ $\left.\left.\mathrm{MHz}_{\mathrm{CDCl}}\right)\right) \delta 160.84\left(\mathrm{~d},{ }^{2} J_{\mathrm{C}-\mathrm{P}}=7.4 \mathrm{~Hz}, \mathrm{C}-\mathrm{OH}\right), 160.39,140.00,137.21\left(\mathrm{~d}, J_{\mathrm{C}-\mathrm{P}}=2.0 \mathrm{~Hz}\right)$, $130.99\left(\mathrm{~d}, J_{\mathrm{C}-\mathrm{P}}=13.4 \mathrm{~Hz}\right), 129.84,128.37,127.18,126.64\left(\mathrm{~d}, J_{\mathrm{C}-\mathrm{P}}=6.9 \mathrm{~Hz}\right), 125.67\left(\mathrm{~d}, J_{\mathrm{C}-\mathrm{P}}\right.$ $=1.4 \mathrm{~Hz}), 119.75\left(\mathrm{~d}, J_{\mathrm{C}-\mathrm{P}}=13.9 \mathrm{~Hz}\right), 117.32,105.13,96.57\left(\mathrm{~d},{ }^{1} J_{\mathrm{C}-\mathrm{P}}=183.9 \mathrm{~Hz}\right), 55.12$, $52.83\left(\mathrm{~d},{ }^{2} J_{\mathrm{C}-\mathrm{P}}=4.7 \mathrm{~Hz},\{\mathrm{PO}\} \mathrm{OCH}_{3} \mathrm{x} 2\right) ;{ }^{31} \mathrm{P} \mathrm{NMR}\left(162 \mathrm{MHz}, \mathrm{CDCl}_{3}\right) \delta 27.28 ; \mathrm{HRMS}$ for $\mathrm{C}_{19} \mathrm{H}_{19} \mathrm{O} 5 \mathrm{P}$ : calcd. $\left(\mathrm{MH}^{+}\right)$: 359.1043, found: 359.1035

\section{Dimethyl (6-chloro-1-hydroxy-4-phenylnaphthalen-2-yl)phosphonate (3c)}

Brown viscous liquid; isolated yield $78 \%(28 \mathrm{mg}) . R_{f} 0.50$ (10\% EtOAc/hexane); ${ }^{1} \mathrm{H}$ NMR $\left(400 \mathrm{MHz}, \mathrm{CDCl}_{3}\right) \delta 11.10(\mathrm{~s}, 1 \mathrm{H}), 8.33(\mathrm{~d}, J=8.9 \mathrm{~Hz}, 1 \mathrm{H}), 7.72(\mathrm{~d}, J=2.0 \mathrm{~Hz}, 1 \mathrm{H}), 7.40-$ $7.44(\mathrm{~m}, 3 \mathrm{H}), 7.34-7.38(\mathrm{~m}, 3 \mathrm{H}), 7.16\left(\mathrm{~d},{ }^{3} J_{\mathrm{H}-\mathrm{P}}=13.3 \mathrm{~Hz}, 1 \mathrm{H}\right), 3.72\left(\mathrm{~d},{ }^{3} J_{\mathrm{H}-\mathrm{P}}=11.5 \mathrm{~Hz}, 6 \mathrm{H}\right)$; ${ }^{13} \mathrm{C} \mathrm{NMR}\left(100 \mathrm{MHz}, \mathrm{CDCl}_{3}\right) \delta 160.74\left(\mathrm{~d},{ }^{2} J_{\mathrm{C}-\mathrm{P}}=7.4 \mathrm{~Hz}, \mathrm{C}-\mathrm{OH}\right), 139.18,136.29\left(\mathrm{~d}, J_{\mathrm{C}-\mathrm{P}}=\right.$ $2.4 \mathrm{~Hz}), 135.88,131.34\left(\mathrm{~d}, J_{\mathrm{C}-\mathrm{P}}=13.3 \mathrm{~Hz}\right), 130.04,128.59,127.61,127.18\left(\mathrm{~d}, J_{\mathrm{C}-\mathrm{P}}=6.6 \mathrm{~Hz}\right)$, 
$126.77,125.79\left(\mathrm{~d}, J_{\mathrm{C}-\mathrm{P}}=1.4 \mathrm{~Hz}\right), 124.88,123.29\left(\mathrm{~d}, J_{\mathrm{C}-\mathrm{P}}=13.9 \mathrm{~Hz}\right), 99.29\left(\mathrm{~d},{ }^{1} J_{\mathrm{C}-\mathrm{P}}=182.8\right.$ $\mathrm{Hz}), 53.09\left(\mathrm{~d},{ }^{2} J_{\mathrm{C}-\mathrm{P}}=4.9 \mathrm{~Hz},\{\mathrm{PO}\} \mathrm{OCH}_{3} \times 2\right) ;{ }^{31} \mathrm{P} \mathrm{NMR}\left(162 \mathrm{MHz}, \mathrm{CDCl}_{3}\right) \delta 26.18 ; \mathrm{HRMS}$ for $\mathrm{C}_{18} \mathrm{H}_{16} \mathrm{ClO}_{4} \mathrm{P}$ : calcd. $\left(\mathrm{MH}^{+}\right)$: 363.0547 , found: 363.0543

\section{Dimethyl (6-fluoro-1-hydroxy-4-phenylnaphthalen-2-yl)phosphonate (3d)}

White solid; isolated yield 76\% (27 mg). $R_{f} 0.50$ (10\% EtOAc/hexane); Mp 101-103 ${ }^{\circ} \mathrm{C} ;{ }^{1} \mathrm{H}$ $\operatorname{NMR}\left(500 \mathrm{MHz}, \mathrm{CDCl}_{3}\right) \delta 11.10(\mathrm{~s}, 1 \mathrm{H}), 8.39(\mathrm{dd}, J=9.2 \mathrm{~Hz}, 6.0 \mathrm{~Hz}, 1 \mathrm{H}), 7.31-7.40(\mathrm{~m}$, $6 \mathrm{H}), 7.17-7.21(\mathrm{~m}, 1 \mathrm{H}), 7.15\left(\mathrm{~d},{ }^{3} J_{\mathrm{H}-\mathrm{P}}=13.0 \mathrm{~Hz}, 1 \mathrm{H}\right), 3.70\left(\mathrm{~d},{ }^{3} J_{\mathrm{H}-\mathrm{P}}=11.5 \mathrm{~Hz}, 6 \mathrm{H}\right) ;{ }^{13} \mathrm{C}$ $\operatorname{NMR}\left(100 \mathrm{MHz}, \mathrm{CDCl}_{3}\right) \delta 163.24\left(\mathrm{~d},{ }^{1} J_{\mathrm{C}-\mathrm{F}}=248.1 \mathrm{~Hz}\right), 160.86\left(\mathrm{~d},{ }^{2} J_{\mathrm{C}-\mathrm{P}}=7.4 \mathrm{~Hz}, \mathrm{C}-\mathrm{OH}\right)$, $139.38,137.17\left(\mathrm{dd}, J_{\mathrm{C}-\mathrm{P}}=2.1 \mathrm{~Hz}, J_{\mathrm{C}-\mathrm{F}}=9.4 \mathrm{~Hz}\right), 131.58\left(\mathrm{dd}, J_{\mathrm{C}-\mathrm{P}}=13.5 \mathrm{~Hz}, J_{\mathrm{C}-\mathrm{F}}=4.8 \mathrm{~Hz}\right)$, $129.96,128.58,127.57,127.14\left(J_{\mathrm{C}-\mathrm{P}}=6.6 \mathrm{~Hz}\right), 127.01\left(\mathrm{~d}, J_{\mathrm{C}-\mathrm{F}}=9.6 \mathrm{~Hz}\right), 121.94\left(\mathrm{~d}, J_{\mathrm{C}-\mathrm{P}}=\right.$ $13.9 \mathrm{~Hz}), 115.81\left(\mathrm{~d}, J_{\mathrm{C}-\mathrm{F}}=24.6 \mathrm{~Hz}\right), 109.88\left(\mathrm{~d}, J_{\mathrm{C}-\mathrm{F}}=22.3 \mathrm{~Hz}\right), 98.38\left(\mathrm{~d},{ }^{1} J_{\mathrm{C}-\mathrm{P}}=183.3 \mathrm{~Hz}\right)$, $53.06\left(\mathrm{~d},{ }^{2} J_{\mathrm{C}-\mathrm{P}}=4.9 \mathrm{~Hz},\{\mathrm{PO}\} \mathrm{OCH}_{3} \times 2\right) ;{ }^{31} \mathrm{P} \mathrm{NMR}\left(162 \mathrm{MHz}, \mathrm{CDCl}_{3}\right) \delta 26.53$; HRMS for $\mathrm{C}_{18} \mathrm{H}_{16} \mathrm{FO}_{4} \mathrm{P}$ : calcd. $\left(\mathrm{MH}^{+}\right)$: 347.0843 , found: 347.0840

\section{Dimethyl (1-hydroxy-4-phenyl-6-(trifluoromethyl)naphthalen-2-yl)phosphonate (3e)}

Colorless viscous liquid; isolated yield $70 \%(28 \mathrm{mg}) . R_{f} 0.50\left(10 \%\right.$ EtOAc/hexane); ${ }^{1} \mathrm{H}$ NMR $\left(400 \mathrm{MHz}, \mathrm{CDCl}_{3}\right) \delta 11.20(\mathrm{~s}, 1 \mathrm{H}), 8.53(\mathrm{~d}, J=8.8 \mathrm{~Hz}, 1 \mathrm{H}), 8.08(\mathrm{~s}, 1 \mathrm{H}), 7.66(\mathrm{dd}, J=8.8$ $\mathrm{Hz}, 1.3 \mathrm{~Hz}, 1 \mathrm{H}), 7.37-7.47(\mathrm{~m}, 5 \mathrm{H}), 7.27\left(\mathrm{dd},{ }^{3} J_{\mathrm{H}-\mathrm{P}}=13.0 \mathrm{~Hz}, 1.2 \mathrm{~Hz}, 1 \mathrm{H}\right), 3.75\left(\mathrm{~d},{ }^{3} J_{\mathrm{H}-\mathrm{P}}=\right.$ $11.5 \mathrm{~Hz}, 6 \mathrm{H}) ;{ }^{13} \mathrm{C} \mathrm{NMR}\left(125 \mathrm{MHz}, \mathrm{CDCl}_{3}\right) \delta 160.42\left(\mathrm{~d},{ }^{2} J_{\mathrm{C}-\mathrm{P}}=7.2 \mathrm{~Hz}, \mathrm{C}-\mathrm{OH}\right), 138.88$, $134.51\left(\mathrm{~d}, J_{\mathrm{C}-\mathrm{P}}=2.0 \mathrm{~Hz}\right), 132.75\left(\mathrm{~d}, J_{\mathrm{C}-\mathrm{P}}=13.2 \mathrm{~Hz}\right), 130.89\left(\mathrm{q}, J_{\mathrm{C}-\mathrm{F}}=32.0 \mathrm{~Hz}\right), 130.03$, 128.70, 127.81, $127.32\left(\mathrm{~d}, J_{\mathrm{C}-\mathrm{P}}=6.6 \mathrm{~Hz}\right), 126.47\left(\mathrm{~d}, J_{\mathrm{C}-\mathrm{P}}=14.0 \mathrm{~Hz}\right), 125.30\left(\mathrm{~d}, J_{\mathrm{C}-\mathrm{P}}=1.5\right.$ $\mathrm{Hz}), 124.10\left(\mathrm{q},{ }^{1} J_{\mathrm{C}-\mathrm{F}}=270.5 \mathrm{~Hz}\right), 123.45\left(\mathrm{q}, J_{\mathrm{C}-\mathrm{F}}=4.4 \mathrm{~Hz}\right), 121.58\left(\mathrm{q}\right.$ appearing as d, $J_{\mathrm{C}-\mathrm{F}}=$ $2.0 \mathrm{~Hz}), 101.17\left(\mathrm{~d},{ }^{1} J_{\mathrm{C}-\mathrm{P}}=181.3 \mathrm{~Hz}\right), 53.17\left(\mathrm{~d},{ }^{2} J_{\mathrm{C}-\mathrm{P}}=5.1 \mathrm{~Hz},\{\mathrm{PO}\} \mathrm{OCH}_{3} \times 2\right) ;{ }^{31} \mathrm{P} \mathrm{NMR}$ $\left(162 \mathrm{MHz}, \mathrm{CDCl}_{3}\right) \delta$ 25.64; HRMS for $\mathrm{C}_{19} \mathrm{H}_{16} \mathrm{~F}_{3} \mathrm{O}_{4} \mathrm{P}$ : calcd. $\left(\mathrm{MH}^{+}\right)$: 397.0811, found: 397.0804

\section{Dimethyl (1-hydroxy-4-phenylanthracen-2-yl)phosphonate (3f)}

White solid; isolated yield 70\% (27 mg). $R_{f} 0.50$ (10\% EtOAc/hexane); Mp 110-111 ${ }^{\circ} \mathrm{C} ;{ }^{1} \mathrm{H}$ NMR $\left(300 \mathrm{MHz}, \mathrm{CDCl}_{3}\right) \delta 11.00(\mathrm{~s}, 1 \mathrm{H}), 7.81-7.87(\mathrm{~m}, 2 \mathrm{H}), 7.73(\mathrm{~d}, J=8.7 \mathrm{~Hz}, 1 \mathrm{H}), 7.42-$ $7.50(\mathrm{~m}, 4 \mathrm{H}), 7.36-7.39(\mathrm{~m}, 2 \mathrm{H}), 7.31\left(\mathrm{~d},{ }^{3} J_{\mathrm{H}-\mathrm{P}}=13.5 \mathrm{~Hz}, 1 \mathrm{H}\right), 7.08-7.13(\mathrm{~m}, 1 \mathrm{H}), 3.80(\mathrm{~d}$, $\left.{ }^{3} J_{\mathrm{H}-\mathrm{P}}=11.5 \mathrm{~Hz}, 6 \mathrm{H}\right) ;{ }^{13} \mathrm{C} \mathrm{NMR}\left(100 \mathrm{MHz}, \mathrm{CDCl}_{3}\right) \delta 160.01\left(\mathrm{~d},{ }^{2} J_{\mathrm{C}-\mathrm{P}}=7.3 \mathrm{~Hz}, \mathrm{C}-\mathrm{OH}\right)$, $144.60,134.74,133.56\left(\mathrm{~d}, J_{\mathrm{C}-\mathrm{P}}=2.6 \mathrm{~Hz}\right), 132.16\left(\mathrm{~d}, J_{\mathrm{C}-\mathrm{P}}=13.2 \mathrm{~Hz}\right), 130.33\left(\mathrm{~d}, J_{\mathrm{C}-\mathrm{P}}=6.5\right.$ Hz), 129.70, 129.29, 129.05, 128.71, 128.48, 127.68, 127.20, 127.16, 124.94, 123.90 (d, $J_{\mathrm{C}-\mathrm{P}}$ $=13.0 \mathrm{~Hz}), 120.84\left(\mathrm{~d}, J_{\mathrm{C}-\mathrm{P}}=0.9 \mathrm{~Hz}\right), 100.85\left(\mathrm{~d},{ }^{1} J_{\mathrm{C}-\mathrm{P}}=182.9 \mathrm{~Hz}\right), 53.14\left(\mathrm{~d},{ }^{2} J_{\mathrm{C}-\mathrm{P}}=4.8 \mathrm{~Hz}\right.$, $\left.\{\mathrm{PO}\} \mathrm{OCH}_{3} \times 2\right) ;{ }^{31} \mathrm{P} \mathrm{NMR}\left(162 \mathrm{MHz}, \mathrm{CDCl}_{3}\right) \delta 25.98$; HRMS for $\mathrm{C}_{22} \mathrm{H}_{19} \mathrm{O}_{4} \mathrm{P}:$ calcd. $\left(\mathrm{MH}^{+}\right)$: 379.1094, found: 379.1090

\section{Dimethyl (1-hydroxy-6,7-dimethoxy-4-phenylnaphthalen-2-yl)phosphonate (3g)}

White solid; isolated yield 47\% (18 mg). $R_{f} 0.50\left(10 \%\right.$ EtOAc/hexane); Mp $154-156{ }^{\circ} \mathrm{C} ;{ }^{1} \mathrm{H}$ NMR $\left(300 \mathrm{MHz}, \mathrm{CDCl}_{3}\right) \delta 10.96(\mathrm{~d}, J=0.9 \mathrm{~Hz}, 1 \mathrm{H}), 7.66(\mathrm{~s}, 1 \mathrm{H}), 7.31-7.40(\mathrm{~m}, 5 \mathrm{H}), 7.10$ $(\mathrm{s}, 1 \mathrm{H}), 7.01\left(\mathrm{~d},{ }^{3} J_{\mathrm{H}-\mathrm{P}}=13.1 \mathrm{~Hz}, 1 \mathrm{H}\right), 3.98(\mathrm{~s}, 3 \mathrm{H}), 3.75(\mathrm{~s}, 3 \mathrm{H}), 3.70\left(\mathrm{~d},{ }^{3} J_{\mathrm{H}-\mathrm{P}}=11.5 \mathrm{~Hz}, 6 \mathrm{H}\right)$; ${ }^{13} \mathrm{C}$ NMR $\left(75 \mathrm{MHz}, \mathrm{CDCl}_{3}\right) \delta 159.42\left(\mathrm{~d},{ }^{2} J_{\mathrm{C}-\mathrm{P}}=7.5 \mathrm{~Hz}, \mathrm{C}-\mathrm{OH}\right), 151.88,149.25,140.31$, $131.65\left(\mathrm{~d}, J_{\mathrm{C}-\mathrm{P}}=2.3 \mathrm{~Hz}\right), 130.94\left(\mathrm{~d}, J_{\mathrm{C}-\mathrm{P}}=13.7 \mathrm{~Hz}\right), 129.92,128.49,127.28,124.67\left(\mathrm{~d}, J_{\mathrm{C}-\mathrm{P}}\right.$ $=6.6 \mathrm{~Hz}), 120.02\left(\mathrm{~d}, J_{\mathrm{C}-\mathrm{P}}=14.3 \mathrm{~Hz}\right), 105.11,102.71\left(\mathrm{~d}, J_{\mathrm{C}-\mathrm{P}}=2.1 \mathrm{~Hz}\right), 97.54\left(\mathrm{~d},{ }^{1} J_{\mathrm{C}-\mathrm{P}}=\right.$ $182.9 \mathrm{~Hz}), 56.09,55.80,52.97\left(\mathrm{~d},{ }^{2} J_{\mathrm{C}-\mathrm{P}}=4.7 \mathrm{~Hz},\{\mathrm{PO}\} \mathrm{OCH}_{3} \times 2\right) ;{ }^{31} \mathrm{P}$ NMR $(121.5 \mathrm{MHz}$, $\left.\mathrm{CDCl}_{3}\right) \delta 27.39$; HRMS for $\mathrm{C}_{20} \mathrm{H}_{21} \mathrm{O} 6 \mathrm{P}$ : calcd. $\left(\mathrm{MH}^{+}\right)$: 389.1149 , found: 389.1155 


\section{Dimethyl (1-hydroxy-5,6-dimethoxy-4-phenylnaphthalen-2-yl)phosphonate (3g')}

White solid; isolated yield 33\% (13 mg). $R_{f} 0.50$ (10\% EtOAc/hexane); Mp $141-143{ }^{\circ} \mathrm{C} ;{ }^{1} \mathrm{H}$ NMR $\left(300 \mathrm{MHz}, \mathrm{CDCl}_{3}\right) \delta 11.03(\mathrm{~d}, J=1.0 \mathrm{~Hz}, 1 \mathrm{H}), 8.23(\mathrm{~d}, J=9.2 \mathrm{~Hz}, 1 \mathrm{H}), 7.23-7.31(\mathrm{~m}$, $6 \mathrm{H}), 6.96\left(\mathrm{~d},{ }^{3} J_{\mathrm{H}-\mathrm{P}}=12.9 \mathrm{~Hz}, 1 \mathrm{H}\right), 3.90(\mathrm{~s}, 3 \mathrm{H}), 3.70\left(\mathrm{~d},{ }^{3} J_{\mathrm{H}-\mathrm{P}}=11.5 \mathrm{~Hz}, 6 \mathrm{H}\right), 3.05(\mathrm{~s}, 3 \mathrm{H})$; ${ }^{13} \mathrm{C}$ NMR $\left(75 \mathrm{MHz}, \mathrm{CDCl}_{3}\right) \delta 161.10\left(\mathrm{~d},{ }^{2} \mathrm{~J}_{\mathrm{C}-\mathrm{P}}=7.4 \mathrm{~Hz}, \mathrm{C}-\mathrm{OH}\right), 153.11,143.90,143.42$, $130.85\left(\mathrm{~d}, J_{\mathrm{C}-\mathrm{P}}=2.3 \mathrm{~Hz}\right), 129.47,129.42\left(\mathrm{~d}, J_{\mathrm{C}-\mathrm{P}}=13.2 \mathrm{~Hz}\right), 128.65\left(\mathrm{~d}, J_{\mathrm{C}-\mathrm{P}}=6.9 \mathrm{~Hz}\right)$, 126.80, 126.05, $120.98\left(\mathrm{~d}, J_{\mathrm{C}-\mathrm{P}}=13.7 \mathrm{~Hz}\right), 120.95\left(\mathrm{~d}, J_{\mathrm{C}-\mathrm{P}}=1.6 \mathrm{~Hz}\right), 113.36,96.34\left(\mathrm{~d},{ }^{1} J_{\mathrm{C}-\mathrm{P}}=\right.$ $184.6 \mathrm{~Hz}), 60.38,56.33,52.99\left(\mathrm{~d},{ }^{2} J_{\mathrm{C}-\mathrm{P}}=5.0 \mathrm{~Hz},\{\mathrm{PO}\} \mathrm{OCH}_{3} \times 2\right) ;{ }^{31} \mathrm{P}$ NMR $(121.5 \mathrm{MHz}$, $\left.\mathrm{CDCl}_{3}\right) \delta 26.83$; HRMS for $\mathrm{C}_{20} \mathrm{H}_{21} \mathrm{O} 6 \mathrm{P}$ : calcd. $\left(\mathrm{MH}^{+}\right)$: 389.1149 , found: 389.1153

\section{Dimethyl (4-hydroxy-1-phenyldibenzo[b,d]furan-3-yl)phosphonate (3h)}

White solid; isolated yield 59\% (22 mg). $R_{f} 0.50$ (20\% EtOAc/hexane); Mp 151-153 ${ }^{\circ} \mathrm{C} ;{ }^{1} \mathrm{H}$ NMR (400 MHz, $\left.\mathrm{CDCl}_{3}\right) \delta 10.61(\mathrm{~s}, 1 \mathrm{H}), 7.61(\mathrm{~d}, J=8.3 \mathrm{~Hz}, 1 \mathrm{H}), 7.50-7.60(\mathrm{~m}, 2 \mathrm{H}), 7.39-$ $7.48(\mathrm{~m}, 5 \mathrm{H}), 7.12\left(\mathrm{~d},{ }^{3} J_{\mathrm{H}-\mathrm{P}}=13.9 \mathrm{~Hz}, 1 \mathrm{H}\right), 7.07-7.11(\mathrm{~m}, 1 \mathrm{H}), 3.75\left(\mathrm{~d},{ }^{3} J_{\mathrm{H}-\mathrm{P}}=11.5 \mathrm{~Hz}, 6 \mathrm{H}\right)$; ${ }^{13} \mathrm{C}$ NMR $\left(75 \mathrm{MHz}, \mathrm{CDCl}_{3}\right) \delta 157.12,148.44\left(\mathrm{~d},{ }^{2} J_{\mathrm{C}-\mathrm{P}}=8.4 \mathrm{~Hz}, \mathrm{C}-\mathrm{OH}\right), 138.78,129.54$, 129.33, 129.10, 128.64, 128.45, 128.03, 127.96, 125.49 (d, $\left.J_{\mathrm{C}-\mathrm{P}}=6.8 \mathrm{~Hz}\right), 123.42,122.88(\mathrm{~d}$, $\left.J_{\mathrm{C}-\mathrm{P}}=12.0 \mathrm{~Hz}\right), 112.34,104.65\left(\mathrm{~d},{ }^{1} J_{\mathrm{C}-\mathrm{P}}=181.3 \mathrm{~Hz}\right), 53.22\left(\mathrm{~d},{ }^{2} J_{\mathrm{C}-\mathrm{P}}=4.8 \mathrm{~Hz},\left\{\mathrm{PO}_{0} \mathrm{OCH}_{3} \mathrm{X}\right.\right.$ 2); ${ }^{31} \mathrm{P}$ NMR (162 $\left.\mathrm{MHz}, \mathrm{CDCl}_{3}\right) \delta 25.41$; HRMS for $\mathrm{C}_{20} \mathrm{H}_{17} \mathrm{O}_{5} \mathrm{P}$ : calcd. $\left(\mathrm{MH}^{+}\right)$: 369.0886, found: 369.0887

\section{Dimethyl (1-hydroxy-4-phenyl-9H-carbazol-2-yl)phosphonate (3i)}

Colorless viscous liquid; isolated yield $52 \%$ (19 mg). $R_{f} 0.50$ (20\% EtOAc/hexane); ${ }^{1} \mathrm{H}$ NMR $\left(400 \mathrm{MHz}, \mathrm{CDCl}_{3}\right) \delta 10.57(\mathrm{~s}, 1 \mathrm{H}), 8.70(\mathrm{~s}, 1 \mathrm{H}), 7.54(\mathrm{~d}, J=6.8 \mathrm{~Hz}, 2 \mathrm{H}), 7.39-7.46(\mathrm{~m}, 5 \mathrm{H})$, $7.34(\mathrm{t}, J=7.4 \mathrm{~Hz}, 1 \mathrm{H}), 6.92-6.95\left(\mathrm{~d},{ }^{3} J_{\mathrm{H}-\mathrm{P}}=12.2 \mathrm{~Hz}, 1 \mathrm{H}\right.$ merged with $\left.\mathrm{s}, 1 \mathrm{H}\right), 3.73\left(\mathrm{~d},{ }^{3} J_{\mathrm{H}-\mathrm{P}}=\right.$ $11.5 \mathrm{~Hz}, 6 \mathrm{H}) ;{ }^{13} \mathrm{C}$ NMR $\left(75 \mathrm{MHz}, \mathrm{CDCl}_{3}\right) \delta 148.80\left(\mathrm{~d},{ }^{2} J_{\mathrm{C}-\mathrm{P}}=8.3 \mathrm{~Hz}, \mathrm{C}-\mathrm{OH}\right), 140.12\left(\mathrm{~d}, J_{\mathrm{C}-\mathrm{P}}\right.$ $=9.8 \mathrm{~Hz}), 129.88\left(\mathrm{~d}, J_{\mathrm{C}-\mathrm{P}}=15.1 \mathrm{~Hz}\right), 129.37,128.78\left(\mathrm{~d}, J_{\mathrm{C}-\mathrm{P}}=18.5 \mathrm{~Hz}\right), 128.43,127.54$, $126.89,126.05,122.91,122.75,121.49\left(\mathrm{~d}, J_{\mathrm{C}-\mathrm{P}}=7.0 \mathrm{~Hz}\right), 119.49,114.93,111.32,100.73(\mathrm{~d}$, $\left.{ }^{1} J_{\mathrm{C}-\mathrm{P}}=182.1 \mathrm{~Hz}\right), 53.13\left(\mathrm{~d},{ }^{2} J_{\mathrm{C}-\mathrm{P}}=4.8 \mathrm{~Hz},\{\mathrm{PO}\} \mathrm{OCH}_{3} \mathrm{x} 2\right) ;{ }^{31} \mathrm{P} \mathrm{NMR}\left(162 \mathrm{MHz}, \mathrm{CDCl}_{3}\right) \delta$ 26.95; HRMS for $\mathrm{C}_{20} \mathrm{H}_{18} \mathrm{NO}_{4} \mathrm{P}$ : calcd. $\left(\mathrm{MH}^{+}\right)$: 368.1046 , found: 368.1044

\section{Dimethyl (7-hydroxy-4-phenylbenzofuran-6-yl)phosphonate (3j)}

White solid; isolated yield 57\% (18 mg). $R_{f} 0.50$ (10\% EtOAc/hexane); Mp $129-131{ }^{\circ} \mathrm{C} ;{ }^{1} \mathrm{H}$ NMR $\left(500 \mathrm{MHz}, \mathrm{CDCl}_{3}\right) \delta 10.70(\mathrm{~s}, 1 \mathrm{H}), 7.71(\mathrm{~d}, J=7.5 \mathrm{~Hz}, 2 \mathrm{H}), 7.59(\mathrm{~s}, 1 \mathrm{H}), 7.41(\mathrm{t}, J=$ $7.2 \mathrm{~Hz}, 2 \mathrm{H}), 7.30-7.32(\mathrm{~m}, 2 \mathrm{H}), 6.96(\mathrm{~s}, 1 \mathrm{H}), 3.72\left(\mathrm{~d},{ }^{3} J_{\mathrm{H}-\mathrm{P}}=11.5 \mathrm{~Hz}, 6 \mathrm{H}\right) ;{ }^{13} \mathrm{C}$ NMR $(100$ $\left.\mathrm{MHz} \mathrm{CDCl}_{3}\right) \delta 157.59\left(\mathrm{~d},{ }^{2} J_{\mathrm{C}-\mathrm{P}}=9.1 \mathrm{~Hz}, \mathrm{C}-\mathrm{OH}\right), 156.62,144.38,135.43,128.69,128.15$, $127.60,126.02\left(\mathrm{~d}, J_{\mathrm{C}-\mathrm{P}}=8.2 \mathrm{~Hz}\right), 119.46\left(\mathrm{~d}, J_{\mathrm{C}-\mathrm{P}}=14.8 \mathrm{~Hz}\right), 118.05\left(\mathrm{~d}, J_{\mathrm{C}-\mathrm{P}}=16.0 \mathrm{~Hz}\right)$, $104.93,99.93\left(\mathrm{~d},{ }^{1} J_{\mathrm{C}-\mathrm{P}}=182.8 \mathrm{~Hz}\right), 53.01\left(\mathrm{~d},{ }^{2} J_{\mathrm{C}-\mathrm{P}}=4.5 \mathrm{~Hz},\{\mathrm{PO}\} \mathrm{OCH}_{3} \times 2\right) ;{ }^{31} \mathrm{P} \mathrm{NMR}(162$ $\left.\mathrm{MHz}_{\mathrm{CDCl}}\right) \delta 26.83$; HRMS for $\mathrm{C}_{16} \mathrm{H}_{15} \mathrm{O} 5 \mathrm{P}$ : calcd. $\left(\mathrm{MH}^{+}\right): 319.0730$, found: 319.0727

\section{Dimethyl (7-hydroxy-4-phenylbenzo[b]thiophen-6-yl)phosphonate (3k)}

White solid; isolated yield 62\% (21 mg). $R_{f} 0.50\left(10 \%\right.$ EtOAc/hexane); Mp $171-173{ }^{\circ} \mathrm{C} ;{ }^{1} \mathrm{H}$ NMR $\left(300 \mathrm{MHz} \mathrm{CDCl}_{3}\right) \delta 10.83(\mathrm{~s}, 1 \mathrm{H}), 7.55(\mathrm{dd}, J=5.4 \mathrm{~Hz}, 0.8 \mathrm{~Hz}, 1 \mathrm{H}), 7.35-7.45(\mathrm{~m}$, $5 \mathrm{H}), 7.29-7.33(\mathrm{~m}, 1 \mathrm{H}), 7.18\left(\mathrm{~d},{ }^{3} J_{\mathrm{H}-\mathrm{P}}=13.4 \mathrm{~Hz}, 1 \mathrm{H}\right), 3.71\left(\mathrm{~d},{ }^{3} J_{\mathrm{H}-\mathrm{P}}=11.5 \mathrm{~Hz}, 6 \mathrm{H}\right) ;{ }^{13} \mathrm{C}$ NMR $\left(100 \mathrm{MHz}, \mathrm{CDCl}_{3}\right) \delta 157.71\left(\mathrm{~d},{ }^{2} J_{\mathrm{C}-\mathrm{P}}=8.5 \mathrm{~Hz}, \mathrm{C}-\mathrm{OH}\right), 143.79\left(\mathrm{~d}, J_{\mathrm{C}-\mathrm{P}}=2.1 \mathrm{~Hz}\right)$, $139.88,130.68,130.31\left(\mathrm{~d}, J_{\mathrm{C}-\mathrm{P}}=14.4 \mathrm{~Hz}\right), 128.99,128.81\left(\mathrm{~d}, J_{\mathrm{C}-\mathrm{P}}=17.2 \mathrm{~Hz}\right), 128.65$, $127.41,126.38\left(\mathrm{~d}, J_{\mathrm{C}-\mathrm{P}}=7.3 \mathrm{~Hz}\right), 124.04,99.86\left(\mathrm{~d},{ }^{1} J_{\mathrm{C}-\mathrm{P}}=182.1 \mathrm{~Hz}\right), 53.12\left(\mathrm{~d},{ }^{2} J_{\mathrm{C}-\mathrm{P}}=4.7\right.$ $\left.\mathrm{Hz},\{\mathrm{PO}\} \mathrm{OCH}_{3} \times 2\right) ;{ }^{31} \mathrm{P} \mathrm{NMR}\left(162 \mathrm{MHz}, \mathrm{CDCl}_{3}\right) \delta 26.51$; HRMS for $\mathrm{C}_{16} \mathrm{H}_{15} \mathrm{O}_{4} \mathrm{PS}$ : calcd. $\left(\mathrm{MH}^{+}\right): 335.0501$, found: 335.0501 
Dimethyl (7-hydroxy-2-methyl-4-phenylbenzo[b]thiophen-6-yl)phosphonate (31)

White solid; isolated yield 59\% (21 mg). $R_{f} 0.50\left(10 \%\right.$ EtOAc/hexane); Mp $177-178{ }^{\circ} \mathrm{C} ;{ }^{1} \mathrm{H}$ NMR $\left(400 \mathrm{MHz}, \mathrm{CDCl}_{3}\right) \delta 10.71(\mathrm{~d}, J=1.0 \mathrm{~Hz}, 1 \mathrm{H}), 7.36-7.44(\mathrm{~m}, 4 \mathrm{H}), 7.28-7.32(\mathrm{~m}, 1 \mathrm{H})$, $7.12\left(\mathrm{~d},{ }^{3} J_{\mathrm{H}-\mathrm{P}}=13.5 \mathrm{~Hz}, 1 \mathrm{H}\right), 7.03(\mathrm{~d}, J=1.1 \mathrm{~Hz}, 1 \mathrm{H}), 3.70\left(\mathrm{~d},{ }^{3} J_{\mathrm{H}-\mathrm{P}}=11.5 \mathrm{~Hz}, 6 \mathrm{H}\right), 2.53(\mathrm{~d}$, $J=1.0 \mathrm{~Hz}, 3 \mathrm{H}) ;{ }^{13} \mathrm{C}$ NMR $\left(100 \mathrm{MHz}, \mathrm{CDCl}_{3}\right) \delta 157.02\left(\mathrm{~d},{ }^{2} J_{\mathrm{C}-\mathrm{P}}=8.7 \mathrm{~Hz}, \mathrm{C}-\mathrm{OH}\right), 145.87$, $144.57,140.08,129.41\left(\mathrm{~d}, J_{\mathrm{C}-\mathrm{P}}=14.5 \mathrm{~Hz}\right), 128.95,128.56,127.98\left(\mathrm{~d}, J_{\mathrm{C}-\mathrm{P}}=17.3 \mathrm{~Hz}\right)$, $127.25,126.41\left(\mathrm{~d}, J_{\mathrm{C}-\mathrm{P}}=7.3 \mathrm{~Hz}\right), 121.96,99.14\left(\mathrm{~d},{ }^{1} J_{\mathrm{C}-\mathrm{P}}=182.5 \mathrm{~Hz}\right), 53.04\left(\mathrm{~d},{ }^{2} J_{\mathrm{C}-\mathrm{P}}=4.6\right.$ $\left.\mathrm{Hz},\{\mathrm{PO}\} \mathrm{OCH}_{3} \times 2\right), 16.45 ;{ }^{31} \mathrm{P}$ NMR $\left(162 \mathrm{MHz}, \mathrm{CDCl}_{3}\right) \delta 26.75$; HRMS for $\mathrm{C}_{17} \mathrm{H}_{17} \mathrm{O}_{4} \mathrm{PS}$ : calcd. $\left(\mathrm{MH}^{+}\right)$: 349.0658, found: 349.0661

\section{Dimethyl (4-hydroxy-7-phenylbenzofuran-5-yl)phosphonate (3m)}

White solid; isolated yield 54\% (17 mg). $R_{f} 0.50$ (10\% EtOAc/hexane); Mp 126-127 ${ }^{\circ} \mathrm{C} ;{ }^{1} \mathrm{H}$ NMR (400 MHz, $\left.\mathrm{CDCl}_{3}\right) \delta 10.71(\mathrm{~s}, 1 \mathrm{H}), 7.70-7.72(\mathrm{~m}, 2 \mathrm{H}), 7.59(\mathrm{~d}, J=2.2 \mathrm{~Hz}, 1 \mathrm{H}), 7.39-$ $7.43(\mathrm{~m}, 2 \mathrm{H}), 7.32\left(\mathrm{~d},{ }^{3} J_{\mathrm{H}-\mathrm{P}}=13.9 \mathrm{~Hz}, 1 \mathrm{H}\right), 7.28-7.33(\mathrm{~m}, 1 \mathrm{H}), 6.97(\mathrm{~d}, J=2.2 \mathrm{~Hz}, 1 \mathrm{H}), 3.72$ $\left(\mathrm{d},{ }^{3} J_{\mathrm{H}-\mathrm{P}}=11.5 \mathrm{~Hz}, 6 \mathrm{H}\right) ;{ }^{13} \mathrm{C} \mathrm{NMR}\left(125 \mathrm{MHz}, \mathrm{CDCl}_{3}\right) \delta 157.59\left(\mathrm{~d},{ }^{2} J_{\mathrm{C}-\mathrm{P}}=8.8 \mathrm{~Hz}, \mathrm{C}-\mathrm{OH}\right)$, $156.65,144.40,135.44,128.70,128.15,127.61,126.03\left(\mathrm{~d}, J_{\mathrm{C}-\mathrm{P}}=8.1 \mathrm{~Hz}\right), 119.47$ (d, $J_{\mathrm{C}-\mathrm{P}}=$ $14.6 \mathrm{~Hz}), 118.07\left(\mathrm{~d}, J_{\mathrm{C}-\mathrm{P}}=16.1 \mathrm{~Hz}\right), 104.93,99.96\left(\mathrm{~d},{ }^{1} J_{\mathrm{C}-\mathrm{P}}=182.4 \mathrm{~Hz}\right), 53.01\left(\mathrm{~d},{ }^{2} J_{\mathrm{C}-\mathrm{P}}=4.5\right.$ $\left.\mathrm{Hz},\{\mathrm{PO}\} \mathrm{OCH}_{3} \times 2\right) ;{ }^{31} \mathrm{P} \mathrm{NMR}\left(162 \mathrm{MHz}, \mathrm{CDCl}_{3}\right) \delta 26.85$; HRMS for $\mathrm{C}_{16} \mathrm{H}_{15} \mathrm{O}_{5} \mathrm{P}$ : calcd. $\left(\mathrm{MH}^{+}\right)$: 319.0730, found: 319.0726

\section{Dimethyl (4-hydroxy-7-phenylbenzo[b]thiophen-5-yl)phosphonate (3n)}

White solid; isolated yield 58\% (20 mg). $R_{f} 0.50\left(10 \%\right.$ EtOAc/hexane); Mp $141-143{ }^{\circ} \mathrm{C} ;{ }^{1} \mathrm{H}$ NMR (400 MHz, $\left.\mathrm{CDCl}_{3}\right) \delta 10.76(\mathrm{~d}, J=1.0 \mathrm{~Hz}, 1 \mathrm{H}), 7.58-7.62(\mathrm{~m}, 3 \mathrm{H}), 7.39-7.43(\mathrm{~m}, 2 \mathrm{H})$, 7.31-7.36 (m, 2H), $7.17\left(\mathrm{~d},{ }^{3} J_{\mathrm{H}-\mathrm{P}}=13.4 \mathrm{~Hz}, 1 \mathrm{H}\right), 3.72\left(\mathrm{~d},{ }^{3} J_{\mathrm{H}-\mathrm{P}}=11.5 \mathrm{~Hz}, 6 \mathrm{H}\right) ;{ }^{13} \mathrm{C}$ NMR $\left(100 \mathrm{MHz} \mathrm{CDCl}_{3}\right) \delta 158.34\left(\mathrm{~d},{ }^{2} J_{\mathrm{C}-\mathrm{P}}=8.1 \mathrm{~Hz}, \mathrm{C}-\mathrm{OH}\right), 145.90,139.55,130.48\left(\mathrm{~d}, J_{\mathrm{C}-\mathrm{P}}=14.6\right.$ $\mathrm{Hz}), 129.04,128.87,128.06,127.93,125.81,125.32\left(\mathrm{~d}, J_{\mathrm{C}-\mathrm{P}}=7.6 \mathrm{~Hz}\right), 121.97,100.64(\mathrm{~d}$, $\left.{ }^{1} J_{\mathrm{C}-\mathrm{P}}=182.0 \mathrm{~Hz}\right), 53.04\left(\mathrm{~d},{ }^{2} J_{\mathrm{C}-\mathrm{P}}=4.5 \mathrm{~Hz},\{\mathrm{PO}\} \mathrm{OCH}_{3} \times 2\right) ;{ }^{31} \mathrm{P} \mathrm{NMR}\left(162 \mathrm{MHz}, \mathrm{CDCl}_{3}\right) \delta$ 26.79; HRMS for $\mathrm{C}_{16} \mathrm{H}_{15} \mathrm{O}_{4} \mathrm{PS}$ : calcd. $\left(\mathrm{MH}^{+}\right)$: 335.0501 , found: 335.0497

\section{Dimethyl (1-hydroxy-4-(p-tolyl)naphthalen-2-yl)phosphonate (3o)}

White solid; isolated yield 74\% (26 mg). $R_{f} 0.50$ (10\% EtOAc/hexane); Mp 107-109 ${ }^{\circ} \mathrm{C} ;{ }^{1} \mathrm{H}$ NMR $\left(500 \mathrm{MHz}, \mathrm{CDCl}_{3}\right) \delta 11.03(\mathrm{~s}, 1 \mathrm{H}), 8.35-8.37(\mathrm{~m}, 1 \mathrm{H}), 7.74-7.76(\mathrm{~m}, 1 \mathrm{H}), 7.42-7.44$ $(\mathrm{m}, 2 \mathrm{H}), 7.24(\mathrm{~d}, J=7.9 \mathrm{~Hz}, 2 \mathrm{H}), 7.17(\mathrm{~d}, J=7.7 \mathrm{~Hz}, 2 \mathrm{H}), 7.10\left(\mathrm{~d},{ }^{3} J_{\mathrm{H}-\mathrm{P}}=13.1 \mathrm{~Hz}, 1 \mathrm{H}\right), 3.67$ $\left(\mathrm{d},{ }^{3} J_{\mathrm{H}-\mathrm{P}}=11.5 \mathrm{~Hz}, 6 \mathrm{H}\right), 2.33(\mathrm{~s}, 3 \mathrm{H}) ;{ }^{13} \mathrm{C} \mathrm{NMR}\left(100 \mathrm{MHz}, \mathrm{CDCl}_{3}\right) \delta 160.71\left(\mathrm{~d},{ }^{2} J_{\mathrm{C}-\mathrm{P}}=7.1\right.$ $\mathrm{Hz}, \mathrm{C}-\mathrm{OH}), 136.95,136.84,135.42\left(\mathrm{~d}, J_{\mathrm{C}-\mathrm{P}}=2.0 \mathrm{~Hz}\right), 132.03\left(\mathrm{~d}, J_{\mathrm{C}-\mathrm{P}}=13.4 \mathrm{~Hz}\right), 129.94$, $129.18,129.02,125.88,125.82,125.60\left(\mathrm{~d}, J_{\mathrm{C}-\mathrm{P}}=6.5 \mathrm{~Hz}\right), 124.94\left(\mathrm{~d}, J_{\mathrm{C}-\mathrm{P}}=13.8 \mathrm{~Hz}\right), 123.80$ $\left(\mathrm{d}, J_{\mathrm{C}-\mathrm{P}}=1.0 \mathrm{~Hz}\right), 98.61\left(\mathrm{~d},{ }^{1} J_{\mathrm{C}-\mathrm{P}}=181.6 \mathrm{~Hz}\right), 52.92\left(\mathrm{~d},{ }^{2} J_{\mathrm{C}-\mathrm{P}}=4.6 \mathrm{~Hz},\{\mathrm{PO}\} \mathrm{OCH}_{3} \mathrm{x} 2\right)$, 21.14; ${ }^{31} \mathrm{P}$ NMR $\left(162 \mathrm{MHz}, \mathrm{CDCl}_{3}\right) \delta 26.88$; HRMS for $\mathrm{C}_{19} \mathrm{H}_{19} \mathrm{O}_{4} \mathrm{P}$ : calcd. $\left(\mathrm{MH}^{+}\right): 343.1094$, found: 343.1097

\section{Dimethyl (4-(4-ethylphenyl)-1-hydroxynaphthalen-2-yl)phosphonate (3p)}

White solid; isolated yield 78\% (28 mg). $R_{f} 0.50$ (10\% EtOAc/hexane); Mp 65-67 ${ }^{\circ} \mathrm{C} ;{ }^{1} \mathrm{H}$ NMR (500 MHz, CDCl 3 ) $\delta 11.03(\mathrm{~s}, 1 \mathrm{H}), 8.39(\mathrm{~d}, J=7.1 \mathrm{~Hz}, 1 \mathrm{H}), 7.79(\mathrm{~d}, J=7.1 \mathrm{~Hz}, 1 \mathrm{H})$, $7.47(\mathrm{br} \mathrm{s}, 2 \mathrm{H}), 7.29(\mathrm{~d}, J=6.8 \mathrm{~Hz}, 2 \mathrm{H}), 7.22(\mathrm{~d}, J=6.8 \mathrm{~Hz}, 2 \mathrm{H}), 7.12\left(\mathrm{~d},{ }^{3} J_{\mathrm{H}-\mathrm{P}}=12.9 \mathrm{~Hz}\right.$, $1 \mathrm{H}), 3.70\left(\mathrm{~d},{ }^{3} J_{\mathrm{H}-\mathrm{P}}=11.5 \mathrm{~Hz}, 6 \mathrm{H}\right), 2.66(\mathrm{q}, J=7.3 \mathrm{~Hz}, 2 \mathrm{H}), 1.24(\mathrm{t}, J=7.3 \mathrm{~Hz}, 3 \mathrm{H}) ;{ }^{13} \mathrm{C}$ NMR $\left(100 \mathrm{MHz} \mathrm{CDCl}_{3}\right) \delta 160.77\left(\mathrm{~d},{ }^{2} J_{\mathrm{C}-\mathrm{P}}=7.3 \mathrm{~Hz}, \mathrm{C}-\mathrm{OH}\right), 143.39,137.15,135.49,132.13$ $\left(\mathrm{d}, J_{\mathrm{C}-\mathrm{P}}=13.7 \mathrm{~Hz}\right), 130.08,129.24,127.88,126.00,125.88,125.72\left(\mathrm{~d}, J_{\mathrm{C}-\mathrm{P}}=6.6 \mathrm{~Hz}\right), 125.00$ $\left(\mathrm{d}, J_{\mathrm{C}-\mathrm{P}}=13.6 \mathrm{~Hz}\right), 123.86\left(\mathrm{~d}, J_{\mathrm{C}-\mathrm{P}}=1.6 \mathrm{~Hz}\right), 98.66\left(\mathrm{~d},{ }^{1} J_{\mathrm{C}-\mathrm{P}}=181.8 \mathrm{~Hz}\right), 53.01\left(\mathrm{~d},{ }^{2} J_{\mathrm{C}-\mathrm{P}}=4.7\right.$ $\mathrm{Hz},\left\{\mathrm{PO}_{\mathrm{OCH}_{3} \times} \times 2\right), 28.64,15.63 ;{ }^{31} \mathrm{P} \mathrm{NMR}\left(162 \mathrm{MHz}, \mathrm{CDCl}_{3}\right) \delta 26.86 ; \mathrm{HRMS}$ for $\mathrm{C}_{20} \mathrm{H}_{21} \mathrm{O}_{4} \mathrm{P}$ : calcd. $\left(\mathrm{MH}^{+}\right)$: 357.1250, found: 357.1249 


\section{Dimethyl (4-(4-(tert-butyl)phenyl)-1-hydroxynaphthalen-2-yl)phosphonate (3q)}

White solid; isolated yield 74\% (29 mg). $R_{f} 0.50\left(10 \%\right.$ EtOAc/hexane); Mp $144-146{ }^{\circ} \mathrm{C} ;{ }^{1} \mathrm{H}$ $\operatorname{NMR}\left(500 \mathrm{MHz}, \mathrm{CDCl}_{3}\right) \delta 11.03(\mathrm{~s}, 1 \mathrm{H}), 8.40(\mathrm{~d}, J=6.6 \mathrm{~Hz}, 1 \mathrm{H}), 7.82(\mathrm{~d}, J=7.0 \mathrm{~Hz}, 1 \mathrm{H})$, $7.48(\mathrm{br} \mathrm{s}, 2 \mathrm{H}), 7.42(\mathrm{~d}, J=6.8 \mathrm{~Hz}, 2 \mathrm{H}), 7.32(\mathrm{~d}, J=6.8 \mathrm{~Hz}, 2 \mathrm{H}), 7.13\left(\mathrm{~d},{ }^{3} J_{\mathrm{H}-\mathrm{P}}=12.9 \mathrm{~Hz}\right.$, $1 \mathrm{H}), 3.70\left(\mathrm{~d},{ }^{3} J_{\mathrm{H}-\mathrm{P}}=11.4 \mathrm{~Hz}, 6 \mathrm{H}\right), 1.32(\mathrm{~s}, 9 \mathrm{H}) ;{ }^{13} \mathrm{C} \mathrm{NMR}\left(100 \mathrm{MHz}, \mathrm{CDCl}_{3}\right) \delta 160.76(\mathrm{~d}$, $\left.{ }^{2} J_{\mathrm{C}-\mathrm{P}}=7.3 \mathrm{~Hz}, \mathrm{C}-\mathrm{OH}\right), 150.25,136.88,135.48\left(\mathrm{~d}, J_{\mathrm{C}-\mathrm{P}}=1.8 \mathrm{~Hz}\right), 132.05\left(\mathrm{~d}, J_{\mathrm{C}-\mathrm{P}}=13.2 \mathrm{~Hz}\right)$, $129.79,129.22,126.05,125.88,125.77\left(\mathrm{~d}, J_{\mathrm{C}-\mathrm{P}}=6.6 \mathrm{~Hz}\right), 125.29,125.00\left(\mathrm{~d}, J_{\mathrm{C}-\mathrm{P}}=13.8 \mathrm{~Hz}\right)$, $123.85\left(\mathrm{~d}, J_{\mathrm{C}-\mathrm{P}}=1.7 \mathrm{~Hz}\right), 98.67\left(\mathrm{~d},{ }^{1} J_{\mathrm{C}-\mathrm{P}}=181.7 \mathrm{~Hz}\right), 53.00\left(\mathrm{~d},{ }^{2} J_{\mathrm{C}-\mathrm{P}}=4.6 \mathrm{~Hz},\{\mathrm{PO}\} \mathrm{OCH}_{3} \mathrm{X}\right.$ 2), 34.63, 31.44; ${ }^{31} \mathrm{P}$ NMR (162 $\left.\mathrm{MHz}, \mathrm{CDCl}_{3}\right) \delta 26.86$; HRMS for $\mathrm{C}_{22} \mathrm{H}_{25} \mathrm{O} 4 \mathrm{P}$ : calcd. $\left(\mathrm{MH}^{+}\right)$: 385.1563 , found: 385.1562

\section{Dimethyl (1-hydroxy-4-(4-methoxyphenyl)naphthalen-2-yl)phosphonate (3r)}

White solid; isolated yield 80\% (29 mg). $R_{f} 0.50$ (10\% EtOAc/hexane); Mp $112-113{ }^{\circ} \mathrm{C} ;{ }^{1} \mathrm{H}$ NMR $\left(500 \mathrm{MHz} \mathrm{CDCl}_{3}\right) \delta 11.02(\mathrm{~s}, 1 \mathrm{H}), 8.39(\mathrm{~d}, J=7.2 \mathrm{~Hz}, 1 \mathrm{H}), 7.77(\mathrm{~d}, J=7.2 \mathrm{~Hz}, 1 \mathrm{H})$, 7.48-7.49 (m, 2H), $7.29(\mathrm{~d}, J=7.4 \mathrm{~Hz}, 2 \mathrm{H}), 7.11\left(\mathrm{~d},{ }^{3} J_{\mathrm{H}-\mathrm{P}}=12.8 \mathrm{~Hz}, 1 \mathrm{H}\right), 6.94(\mathrm{~d}, J=7.4 \mathrm{~Hz}$, $2 \mathrm{H}), 3.81(\mathrm{~s}, 3 \mathrm{H}), 3.71\left(\mathrm{~d},{ }^{3} J_{\mathrm{H}-\mathrm{P}}=11.5 \mathrm{~Hz}, 6 \mathrm{H}\right) ;{ }^{13} \mathrm{C} \mathrm{NMR}\left(100 \mathrm{MHz}, \mathrm{CDCl}_{3}\right) \delta 160.69(\mathrm{~d}$, $\left.{ }^{2} J_{\mathrm{C}-\mathrm{P}}=7.2 \mathrm{~Hz}, \mathrm{C}-\mathrm{OH}\right), 159.01,135.61,132.21,131.77$ (d, $\left.J_{\mathrm{C}-\mathrm{P}}=13.3 \mathrm{~Hz}\right), 131.18,129.24$, $125.90\left(\mathrm{~d}, J_{\mathrm{C}-\mathrm{P}}=6.1 \mathrm{~Hz}\right), 125.63\left(\mathrm{~d}, J_{\mathrm{C}-\mathrm{P}}=6.6 \mathrm{~Hz}\right), 125.01\left(\mathrm{~d}, J_{\mathrm{C}-\mathrm{P}}=13.8 \mathrm{~Hz}\right), 123.88$, $113.83,98.65\left(\mathrm{~d},{ }^{1} J_{\mathrm{C}-\mathrm{P}}=181.6 \mathrm{~Hz}\right), 55.39,53.00\left(\mathrm{~d},{ }^{2} J_{\mathrm{C}-\mathrm{P}}=4.8 \mathrm{~Hz},\{\mathrm{PO}\} \mathrm{OCH} \mathrm{C}_{3} 2\right) ;{ }^{31} \mathrm{P}$ NMR $\left(162 \mathrm{MHz}, \mathrm{CDCl}_{3}\right) \delta$ 26.86; HRMS for $\mathrm{C}_{19} \mathrm{H}_{19} \mathrm{O} 5 \mathrm{P}$ : calcd. $\left(\mathrm{MH}^{+}\right)$: 359.1043, found: 359.1040

\section{Dimethyl (4-(4-bromophenyl)-1-hydroxynaphthalen-2-yl)phosphonate (3s)}

White solid; isolated yield 73\% (30 mg). $R_{f} 0.50$ (10\% EtOAc/hexane); Mp 97-99 ${ }^{\circ} \mathrm{C} ;{ }^{1} \mathrm{H}$ NMR $\left(400 \mathrm{MHz}, \mathrm{CDCl}_{3}\right) \delta 11.11(\mathrm{~s}, 1 \mathrm{H}), 8.39-8.42(\mathrm{~m}, 1 \mathrm{H}), 7.69-7.72(\mathrm{~m}, 1 \mathrm{H}), 7.48-7.53$ $(\mathrm{m}, 4 \mathrm{H}), 7.25(\mathrm{~d}, J=8.4 \mathrm{~Hz}, 2 \mathrm{H}), 7.11\left(\mathrm{~d},{ }^{3} J_{\mathrm{H}-\mathrm{P}}=12.9 \mathrm{~Hz}, 1 \mathrm{H}\right), 3.72\left(\mathrm{~d},{ }^{3} J_{\mathrm{H}-\mathrm{P}}=11.5 \mathrm{~Hz}, 6 \mathrm{H}\right)$; ${ }^{13} \mathrm{C}$ NMR $\left(100 \mathrm{MHz}, \mathrm{CDCl}_{3}\right) \delta 161.16\left(\mathrm{~d},{ }^{2} J_{\mathrm{C}-\mathrm{P}}=7.2 \mathrm{~Hz}, \mathrm{C}-\mathrm{OH}\right), 138.78,135.10\left(\mathrm{~d}, J_{\mathrm{C}-\mathrm{P}}=\right.$ $2.2 \mathrm{~Hz}), 131.79,131.56,130.79\left(\mathrm{~d}, J_{\mathrm{C}-\mathrm{P}}=13.6 \mathrm{~Hz}\right), 129.56,126.12,125.82\left(\mathrm{~d}, J_{\mathrm{C}-\mathrm{P}}=6.6 \mathrm{~Hz}\right)$, $125.53,125.04\left(\mathrm{~d}, J_{\mathrm{C}-\mathrm{P}}=13.8 \mathrm{~Hz}\right), 124.03\left(\mathrm{~d}, J_{\mathrm{C}-\mathrm{P}}=1.4 \mathrm{~Hz}\right), 121.51,98.81\left(\mathrm{~d},{ }^{1} J_{\mathrm{C}-\mathrm{P}}=182.4\right.$ $\mathrm{Hz}), 53.06\left(\mathrm{~d},{ }^{2} J_{\mathrm{C}-\mathrm{P}}=4.7 \mathrm{~Hz},\{\mathrm{PO}\} \mathrm{OCH}_{3} \times 2\right) ;{ }^{31} \mathrm{P} \mathrm{NMR}\left(162 \mathrm{MHz}, \mathrm{CDCl}_{3}\right) \delta 26.52 ; \mathrm{HRMS}$ for $\mathrm{C}_{18} \mathrm{H}_{16} \mathrm{BrO}_{4} \mathrm{P}$ : calcd. $\left(\mathrm{MH}^{+}\right)$: 407.0042, found: 407.0043

\section{Dimethyl (4-(4-fluorophenyl)-1-hydroxynaphthalen-2-yl)phosphonate (3t)}

White solid; isolated yield 75\% (26 mg). $R_{f} 0.50$ (10\% EtOAc/hexane); Mp 121-123 ${ }^{\circ} \mathrm{C} ;{ }^{1} \mathrm{H}$ NMR $\left(500 \mathrm{MHz}, \mathrm{CDCl}_{3}\right) \delta 11.07(\mathrm{~s}, 1 \mathrm{H}), 8.39-8.40(\mathrm{~m}, 1 \mathrm{H}), 7.68-7.69(\mathrm{~m}, 1 \mathrm{H}), 7.49(\mathrm{t}, J=$ $3.9 \mathrm{~Hz}, 2 \mathrm{H}), 7.31-7.33(\mathrm{~m}, 2 \mathrm{H}), 7.06-7.10(\mathrm{~m}, 3 \mathrm{H}), 3.71\left(\mathrm{~d},{ }^{3} \mathrm{~J}_{\mathrm{H}-\mathrm{P}}=11.5 \mathrm{~Hz}, 6 \mathrm{H}\right) ;{ }^{13} \mathrm{C} \mathrm{NMR}$ $\left(100 \mathrm{MHz}, \mathrm{CDCl}_{3}\right) \delta 162.24\left(\mathrm{~d},{ }^{1} J_{\mathrm{C}-\mathrm{F}}=253.2 \mathrm{~Hz}\right), 161.05,135.78\left(\mathrm{~d}, J_{\mathrm{C}-\mathrm{F}}=3.2 \mathrm{~Hz}\right), 135.40$ $\left(\mathrm{d}, J_{\mathrm{C}-\mathrm{P}}=2.1 \mathrm{~Hz}\right), 131.69\left(J_{\mathrm{C}-\mathrm{F}}=7.9 \mathrm{~Hz}\right), 131.01\left(\mathrm{~d}, J_{\mathrm{C}-\mathrm{P}}=13.4 \mathrm{~Hz}\right), 129.45,126.03,125.84$ $\left(\mathrm{d}, J_{\mathrm{C}-\mathrm{P}}=6.5 \mathrm{~Hz}\right), 125.64,125.01\left(\mathrm{~d}, J_{\mathrm{C}-\mathrm{P}}=13.8 \mathrm{~Hz}\right), 123.99,115.30\left(\mathrm{~d}, J_{\mathrm{C}-\mathrm{F}}=21.2 \mathrm{~Hz}\right)$, $98.71\left(\mathrm{~d},{ }^{1} J_{\mathrm{C}-\mathrm{P}}=182.3 \mathrm{~Hz}\right), 53.02\left(\mathrm{~d},{ }^{2} J_{\mathrm{C}-\mathrm{P}}=4.7 \mathrm{~Hz},\{\mathrm{PO}\} \mathrm{OCH}_{3} \times 2\right) ;{ }^{31} \mathrm{P}$ NMR $(162 \mathrm{MHz}$, $\left.\mathrm{CDCl}_{3}\right) \delta$ 26.64; HRMS for $\mathrm{C}_{18} \mathrm{H}_{16} \mathrm{FO}_{4} \mathrm{P}$ : calcd. $\left(\mathrm{MH}^{+}\right)$: 347.0843 , found: 347.0840

\section{Dimethyl (1-hydroxy-4-(2-(trifluoromethyl)phenyl)naphthalen-2-yl)phosphonate (3u)}

White solid; isolated yield 68\% (27 mg). $R_{f} 0.50$ (10\% EtOAc/hexane); Mp 102-104 ${ }^{\circ} \mathrm{C} ;{ }^{1} \mathrm{H}$ NMR $\left(300 \mathrm{MHz}, \mathrm{CDCl}_{3}\right) \delta 11.14(\mathrm{~d}, J=0.9 \mathrm{~Hz}, 1 \mathrm{H}), 8.38-8.41(\mathrm{~m}, 1 \mathrm{H}), 7.76(\mathrm{~d}, J=7.5 \mathrm{~Hz}$, $1 \mathrm{H}), 7.41-7.57(\mathrm{~m}, 4 \mathrm{H}), 7.31(\mathrm{~d}, J=7.3 \mathrm{~Hz}, 1 \mathrm{H}), 7.18-7.22(\mathrm{~m}, 1 \mathrm{H}), 7.08\left(\mathrm{~d},{ }^{3} J_{\mathrm{H}-\mathrm{P}}=12.8 \mathrm{~Hz}\right.$, $1 \mathrm{H}), 3.72\left(\mathrm{~d},{ }^{3} J_{\mathrm{H}-\mathrm{P}}=11.4 \mathrm{~Hz}, 3 \mathrm{H}\right), 3.66\left(\mathrm{~d},{ }^{3} J_{\mathrm{H}-\mathrm{P}}=11.6 \mathrm{~Hz}, 3 \mathrm{H}\right) ;{ }^{13} \mathrm{C} \mathrm{NMR}\left(75 \mathrm{MHz}, \mathrm{CDCl}_{3}\right)$ $\delta 161.55\left(\mathrm{~d},{ }^{2} J_{\mathrm{C}-\mathrm{P}}=7.2 \mathrm{~Hz}, \mathrm{C}-\mathrm{OH}\right), 138.44,136.19,133.08,131.24,130.08\left(\mathrm{q},{ }^{2} J_{\mathrm{C}-\mathrm{F}}=29.0\right.$ $\mathrm{Hz}), 129.98,129.33,128.16\left(\mathrm{~d}, J_{\mathrm{C}-\mathrm{P}}=13.8 \mathrm{~Hz}\right), 127.88,126.27\left(\mathrm{q}, J_{\mathrm{C}-\mathrm{F}}=5.2 \mathrm{~Hz}\right), 125.95$, $125.89\left(\mathrm{~d}, J_{\mathrm{C}-\mathrm{P}}=1.3 \mathrm{~Hz}\right), 124.67\left(\mathrm{~d}, J_{\mathrm{C}-\mathrm{P}}=13.5 \mathrm{~Hz}\right), 124.02\left(\mathrm{q},{ }^{1} J_{\mathrm{C}-\mathrm{F}}=272.4 \mathrm{~Hz}\right), 123.71(\mathrm{~d}$, 
$\left.J_{\mathrm{C}-\mathrm{P}}=1.7 \mathrm{~Hz}\right), 97.86\left(\mathrm{~d},{ }^{1} J_{\mathrm{C}-\mathrm{P}}=180.7 \mathrm{~Hz}\right), 53.00\left(\mathrm{~d},{ }^{2} J_{\mathrm{C}-\mathrm{P}}=4.3 \mathrm{~Hz},\{\mathrm{PO}\} \mathrm{OCH}_{3}\right), 52.92(\mathrm{~d}$, $\left.{ }^{2} J_{\mathrm{C}-\mathrm{P}}=5.0 \mathrm{~Hz},\{\mathrm{PO}\} \mathrm{OCH}_{3}\right) ;{ }^{31} \mathrm{P}$ NMR $\left(162 \mathrm{MHz}, \mathrm{CDCl}_{3}\right) \delta 26.63$; HRMS for $\mathrm{C}_{19} \mathrm{H}_{16} \mathrm{~F}_{3} \mathrm{O}_{4} \mathrm{P}$ : calcd. $\left(\mathrm{MH}^{+}\right)$: 397.0811, found: 397.0805

\section{Dimethyl (1-hydroxy-4-(thiophen-3-yl)naphthalen-2-yl)phosphonate (3v)}

Colorless viscous liquid; isolated yield $72 \%(24 \mathrm{mg}) . R_{f} 0.50$ (10\% EtOAc/hexane); ${ }^{1} \mathrm{H}$ NMR $\left(300 \mathrm{MHz}, \mathrm{CDCl}_{3}\right) \delta 11.07(\mathrm{~s}, 1 \mathrm{H}), 8.38-8.41(\mathrm{~m}, 1 \mathrm{H}), 7.85-7.89(\mathrm{~m}, 1 \mathrm{H}), 7.46-7.55(\mathrm{~m}, 2 \mathrm{H})$, $7.37(\mathrm{dd}, J=4.9 \mathrm{~Hz}, 3.0 \mathrm{~Hz}, 1 \mathrm{H}), 7.27(\mathrm{dd}, J=3.0 \mathrm{~Hz}, 1.3 \mathrm{~Hz}, 1 \mathrm{H}), 7.17-7.21(\mathrm{~m}, 2 \mathrm{H}), 3.71$ $\left(\mathrm{d},{ }^{3} J_{\mathrm{H}-\mathrm{P}}=11.5 \mathrm{~Hz}, 6 \mathrm{H}\right) ;{ }^{13} \mathrm{C} \mathrm{NMR}\left(75 \mathrm{MHz}, \mathrm{CDCl}_{3}\right) \delta 160.96\left(\mathrm{~d},{ }^{2} J_{\mathrm{C}-\mathrm{P}}=7.1 \mathrm{~Hz}, \mathrm{C}-\mathrm{OH}\right)$, $140.21,135.54\left(\mathrm{~d}, J_{\mathrm{C}-\mathrm{P}}=2.1 \mathrm{~Hz}\right), 129.53,129.42,126.80\left(\mathrm{~d}, J_{\mathrm{C}-\mathrm{P}}=13.6 \mathrm{~Hz}\right), 125.98,125.81$, $125.70,125.41,124.99\left(\mathrm{~d}, J_{\mathrm{C}-\mathrm{P}}=13.8 \mathrm{~Hz}\right), 123.89\left(\mathrm{~d}, J_{\mathrm{C}-\mathrm{P}}=1.6 \mathrm{~Hz}\right), 123.40,98.69\left(\mathrm{~d},{ }^{1} J_{\mathrm{C}-\mathrm{P}}=\right.$ $182.0 \mathrm{~Hz}), 52.99$ (d, $\left.{ }^{2} J_{\mathrm{C}-\mathrm{P}}=4.7 \mathrm{~Hz},\{\mathrm{PO}\} \mathrm{OCH}_{3} \times 2\right) ;{ }^{31} \mathrm{P} \mathrm{NMR}\left(121.5 \mathrm{MHz}, \mathrm{CDCl}_{3}\right) \delta 26.70$; HRMS for $\mathrm{C}_{16} \mathrm{H}_{15} \mathrm{O}_{4} \mathrm{PS}$ : calcd. $\left(\mathrm{MH}^{+}\right)$: 335.0501 , found: 335.0496

\section{Dimethyl (4-cyclohexyl-1-hydroxynaphthalen-2-yl)phosphonate (3w)}

White solid; isolated yield 49\% (17 mg). $R_{f} 0.50$ (10\% EtOAc/hexane); Mp 90-92 ${ }^{\circ} \mathrm{C} ;{ }^{1} \mathrm{H}$ NMR (400 MHz, $\left.\mathrm{CDCl}_{3}\right) \delta 10.96(\mathrm{~s}, 1 \mathrm{H}), 8.44(\mathrm{~d}, J=8.3 \mathrm{~Hz}, 1 \mathrm{H}), 8.03(\mathrm{~d}, J=8.5 \mathrm{~Hz}, 1 \mathrm{H})$, 7.61-7.65 (m, 1H), $7.51(\mathrm{t}, J=7.9 \mathrm{~Hz}, 1 \mathrm{H}), 7.10\left(\mathrm{~d},{ }^{3} J_{\mathrm{H}-\mathrm{P}}=13.4 \mathrm{~Hz}, 1 \mathrm{H}\right), 3.76\left(\mathrm{~d},{ }^{3} J_{\mathrm{H}-\mathrm{P}}=11.5\right.$ $\mathrm{Hz}, 6 \mathrm{H}), 3.14-3.19(\mathrm{~m}, 1 \mathrm{H}), 1.99-2.02(\mathrm{~m}, 4 \mathrm{H}), 1.81-1.84(\mathrm{~m}, 1 \mathrm{H}), 1.44-1.59(\mathrm{~m}, 4 \mathrm{H}), 1.29-$ $1.37(\mathrm{~m}, 1 \mathrm{H}) ;{ }^{13} \mathrm{C}$ NMR $\left(100 \mathrm{MHz}, \mathrm{CDCl}_{3}\right) \delta 159.84\left(\mathrm{~d},{ }^{2} J_{\mathrm{C}-\mathrm{P}}=7.3 \mathrm{~Hz}, \mathrm{C}-\mathrm{OH}\right), 135.27\left(\mathrm{~d}, J_{\mathrm{C}-}\right.$ $\mathrm{P}=2.2 \mathrm{~Hz}), 135.25\left(\mathrm{~d}, J_{\mathrm{C}-\mathrm{P}}=12.4 \mathrm{~Hz}\right), 129.03,125.44,125.15\left(\mathrm{~d}, J_{\mathrm{C}-\mathrm{P}}=13.9 \mathrm{~Hz}\right), 124.33(\mathrm{~d}$, $\left.J_{\mathrm{C}-\mathrm{P}}=1.5 \mathrm{~Hz}\right), 123.11,120.88\left(\mathrm{~d}, J_{\mathrm{C}-\mathrm{P}}=6.9 \mathrm{~Hz}\right), 98.29\left(\mathrm{~d},{ }^{1} J_{\mathrm{C}-\mathrm{P}}=181.4 \mathrm{~Hz}\right), 52.87\left(\mathrm{~d},{ }^{2} J_{\mathrm{C}-\mathrm{P}}=\right.$ $\left.4.5 \mathrm{~Hz},\{\mathrm{PO}\} \mathrm{OCH}_{3} \times 2\right), 38.75,34.25,27.22,26.48 ;{ }^{31} \mathrm{P} \mathrm{NMR}\left(162 \mathrm{MHz}, \mathrm{CDCl}_{3}\right) \delta$ 27.57; HRMS for $\mathrm{C}_{18} \mathrm{H}_{23} \mathrm{O}_{4} \mathrm{P}$ : calcd. $\left(\mathrm{MH}^{+}\right)$: 335.1407, found: 335.1397

\section{Dimethyl (4-cyclopropyl-1-hydroxynaphthalen-2-yl)phosphonate (3x)}

Colorless viscous liquid; isolated yield 47\% (14 mg). $R_{f} 0.50$ (10\% EtOAc/hexane); ${ }^{1} \mathrm{H}$ NMR $\left(400 \mathrm{MHz} \mathrm{CDCl}_{3}\right) \delta 10.91(\mathrm{~s}, 1 \mathrm{H}), 8.33(\mathrm{~d}, J=8.3 \mathrm{~Hz}, 1 \mathrm{H}), 8.26(\mathrm{~d}, J=8.4 \mathrm{~Hz}, 1 \mathrm{H}), 7.57-$ $7.61(\mathrm{~m}, 1 \mathrm{H}), 7.45-7.49(\mathrm{~m}, 1 \mathrm{H}), 6.96\left(\mathrm{dd},{ }^{3} J_{\mathrm{H}-\mathrm{P}}=13.0 \mathrm{~Hz}, 1.1 \mathrm{~Hz}, 1 \mathrm{H}\right), 3.68\left(\mathrm{~d},{ }^{3} J_{\mathrm{H}-\mathrm{P}}=11.5\right.$ $\mathrm{Hz}, 6 \mathrm{H}), 2.04-2.09(\mathrm{~m}, 1 \mathrm{H}), 0.90-0.95(\mathrm{~m}, 2 \mathrm{H}), 0.58-0.61(\mathrm{~m}, 2 \mathrm{H}) ;{ }^{13} \mathrm{C}$ NMR $(100 \mathrm{MHz}$, $\left.\mathrm{CDCl}_{3}\right) \delta 160.41\left(\mathrm{~d},{ }^{2} J_{\mathrm{C}-\mathrm{P}}=7.2 \mathrm{~Hz}, \mathrm{C}-\mathrm{OH}\right), 137.22\left(\mathrm{~d}, J_{\mathrm{C}-\mathrm{P}}=2.1 \mathrm{~Hz}\right), 130.57\left(\mathrm{~d}, J_{\mathrm{C}-\mathrm{P}}=13.1\right.$ $\mathrm{Hz}), 129.16,125.80,124.88\left(\mathrm{~d}, J_{\mathrm{C}-\mathrm{P}}=14.1^{`} \mathrm{~Hz}\right), 124.38,124.03$ (d, $\left.J_{\mathrm{C}-\mathrm{P}}=1.4 \mathrm{~Hz}\right), 123.08$ (d, $\left.J_{\mathrm{C}-\mathrm{P}}=6.6 \mathrm{~Hz}\right), 98.01\left(\mathrm{~d},{ }^{1} J_{\mathrm{C}-\mathrm{P}}=181.5 \mathrm{~Hz}\right), 52.89\left(\mathrm{~d},{ }^{2} J_{\mathrm{C}-\mathrm{P}}=4.5 \mathrm{~Hz},\{\mathrm{PO}\} \mathrm{OCH}_{3} \times 2\right), 12.89$, 6.01; ${ }^{31} \mathrm{P}$ NMR $\left(162 \mathrm{MHz}, \mathrm{CDCl}_{3}\right) \delta 27.32$; HRMS for $\mathrm{C}_{15} \mathrm{H}_{17} \mathrm{O}_{4} \mathrm{P}$ : calcd. $\left(\mathrm{MH}^{+}\right): 293.0937$, found: 293.0934

\section{Dimethyl (1-hydroxy-4-(trimethylsilyl)naphthalen-2-yl)phosphonate (3y)}

Colorless viscous liquid; isolated yield $42 \%$ (14 mg). $R_{f} 0.50$ (10\% EtOAc/hexane); ${ }^{1} \mathrm{H}$ NMR $\left(400 \mathrm{MHz}, \mathrm{CDCl}_{3}\right) \delta 11.19(\mathrm{~s}, 1 \mathrm{H}), 8.46(\mathrm{dd}, J=8.4 \mathrm{~Hz}, 1.2 \mathrm{~Hz}, 1 \mathrm{H}), 8.01(\mathrm{~d}, J=8.3 \mathrm{~Hz}$, $1 \mathrm{H}), 7.61-7.65(\mathrm{~m}, 1 \mathrm{H}), 7.51-7.55(\mathrm{~m}, 1 \mathrm{H}), 7.41\left(\mathrm{~d},{ }^{3} J_{\mathrm{H}-\mathrm{P}}=12.3 \mathrm{~Hz}, 1 \mathrm{H}\right), 3.78\left(\mathrm{~d},{ }^{3} J_{\mathrm{H}-\mathrm{P}}=11.5\right.$ $\mathrm{Hz}, 6 \mathrm{H}), 0.43(\mathrm{~s}, 9 \mathrm{H}) ;{ }^{13} \mathrm{C} \mathrm{NMR}\left(100 \mathrm{MHz}^{\mathrm{CDCl}} 3\right) \delta 162.77\left(\mathrm{~d},{ }^{2} J_{\mathrm{C}-\mathrm{P}}=7.9 \mathrm{~Hz}, \mathrm{C}-\mathrm{OH}\right)$, $140.44\left(\mathrm{~d}, J_{\mathrm{C}-\mathrm{P}}=1.5 \mathrm{~Hz}\right), 132.56\left(\mathrm{~d}, J_{\mathrm{C}-\mathrm{P}}=6.1 \mathrm{~Hz}\right), 128.99,128.60\left(\mathrm{~d}, J_{\mathrm{C}-\mathrm{P}}=10.0 \mathrm{~Hz}\right)$, $127.89,125.53\left(\mathrm{~d}, J_{\mathrm{C}-\mathrm{P}}=1.1 \mathrm{~Hz}\right), 124.86\left(\mathrm{~d}, J_{\mathrm{C}-\mathrm{P}}=13.0 \mathrm{~Hz}\right), 124.41\left(\mathrm{~d}, J_{\mathrm{C}-\mathrm{P}}=1.2 \mathrm{~Hz}\right), 98.65$ $\left(\mathrm{d},{ }^{1} J_{\mathrm{C}-\mathrm{P}}=180.2 \mathrm{~Hz}\right), 52.96\left(\mathrm{~d},{ }^{2} J_{\mathrm{C}-\mathrm{P}}=4.5 \mathrm{~Hz},\{\mathrm{PO}\} \mathrm{OCH}_{3} \times 2\right), 0.21 ;{ }^{31} \mathrm{P}$ NMR $(162 \mathrm{MHz}$, $\left.\mathrm{CDCl}_{3}\right) \delta 27.40$; HRMS for $\mathrm{C}_{15} \mathrm{H}_{21} \mathrm{O}_{4} \mathrm{PSi}$ : calcd. $\left(\mathrm{MH}^{+}\right)$: 325.1019 , found: 325.1024

\section{Ethyl 3-(dimethoxyphosphoryl)-4-hydroxy-1-naphthoate (3z)}

Colorless viscous liquid; isolated yield $40 \%$ (13 mg). $R_{f} 0.50$ (10\% EtOAc/hexane); ${ }^{1} \mathrm{H}$ NMR $\left(400 \mathrm{MHz}, \mathrm{CDCl}_{3}\right) \delta 11.58(\mathrm{br} \mathrm{s}, 1 \mathrm{H}), 8.93(\mathrm{~d}, J=8.6 \mathrm{~Hz}, 1 \mathrm{H}), 8.39(\mathrm{~d}, J=8.4 \mathrm{~Hz}, 1 \mathrm{H}), 8.05$ $\left(\mathrm{d},{ }^{3} J_{\mathrm{H}-\mathrm{P}}=13.7 \mathrm{~Hz}, 1 \mathrm{H}\right), 7.65-7.69(\mathrm{~m}, 1 \mathrm{H}), 7.50-7.54(\mathrm{~m}, 1 \mathrm{H}), 4.38(\mathrm{q}, J=7.1 \mathrm{~Hz}, 2 \mathrm{H}), 3.73$ $\left(\mathrm{d},{ }^{3} J_{\mathrm{H}-\mathrm{P}}=11.6 \mathrm{~Hz}, 6 \mathrm{H}\right), 1.38(\mathrm{t}, J=7.1 \mathrm{~Hz}, 3 \mathrm{H}) ;{ }^{13} \mathrm{C} \mathrm{NMR}\left(100 \mathrm{MHz}, \mathrm{CDCl}_{3}\right) \delta 166.60$, 
$164.93\left(\mathrm{~d},{ }^{2} J_{\mathrm{C}-\mathrm{P}}=7.6 \mathrm{~Hz}, \mathrm{C}-\mathrm{OH}\right), 135.14,131.62\left(\mathrm{~d}, J_{\mathrm{C}-\mathrm{P}}=7.3 \mathrm{~Hz}\right), 130.86,126.30,125.96$, $125.01\left(\mathrm{~d}, J_{\mathrm{C}-\mathrm{P}}=12.9 \mathrm{~Hz}\right), 123.93,119.01\left(\mathrm{~d}, J_{\mathrm{C}-\mathrm{P}}=13.0 \mathrm{~Hz}\right), 98.36\left(\mathrm{~d},{ }^{1} J_{\mathrm{C}-\mathrm{P}}=184.1 \mathrm{~Hz}\right)$, $60.97,53.19\left(\mathrm{~d},{ }^{2} J_{\mathrm{C}-\mathrm{P}}=4.5 \mathrm{~Hz},\{\mathrm{PO}\} \mathrm{OCH}_{3} \times 2\right), 12.45 ;{ }^{31} \mathrm{P} \mathrm{NMR}\left(162 \mathrm{MHz}, \mathrm{CDCl}_{3}\right) \delta 25.75$; HRMS for $\mathrm{C}_{15} \mathrm{H}_{17} \mathrm{O}_{6} \mathrm{P}$ : calcd. $\left(\mathrm{MH}^{+}\right)$: 325.0836, found: 325.0841

\section{Dimethyl (1-hydroxy-3,4-diphenylnaphthalen-2-yl)phosphonate (3za)}

White solid; isolated yield 44\% (18 mg). $R_{f} 0.50$ (10\% EtOAc/hexane); Mp 88-90 ${ }^{\circ} \mathrm{C} ;{ }^{1} \mathrm{H}$ $\operatorname{NMR}\left(400 \mathrm{MHz}, \mathrm{CDCl}_{3}\right) \delta 12.38(\mathrm{~d}, J=1.3 \mathrm{~Hz}, 1 \mathrm{H}), 8.44-8.47(\mathrm{~m}, 1 \mathrm{H}), 7.40-7.48(\mathrm{~m}, 2 \mathrm{H})$, 7.27-7.29 (m, 1H), 7.00-7.10 (m, 6H), 6.93-6.97 (m, 4H), $3.42\left(\mathrm{~d},{ }^{3} J_{\mathrm{H}-\mathrm{P}}=11.6 \mathrm{~Hz}, 6 \mathrm{H}\right) ;{ }^{13} \mathrm{C}$ $\operatorname{NMR}\left(100 \mathrm{MHz}, \mathrm{CDCl}_{3}\right) \delta 162.14\left(\mathrm{~d},{ }^{2} J_{\mathrm{C}-\mathrm{P}}=7.6 \mathrm{~Hz}, \mathrm{C}-\mathrm{OH}\right), 139.43\left(\mathrm{~d}, J_{\mathrm{C}-\mathrm{P}}=3.3 \mathrm{~Hz}\right)$, $138.64\left(\mathrm{~d}, J_{\mathrm{C}-\mathrm{P}}=7.2 \mathrm{~Hz}\right), 136.08,131.44,130.67,129.55,127.47,126.63,126.32,125.70$, $124.40\left(\mathrm{~d}, J_{\mathrm{C}-\mathrm{P}}=13.9 \mathrm{~Hz}\right), 123.87,99.09\left(\mathrm{~d},{ }^{1} J_{\mathrm{C}-\mathrm{P}}=181.0 \mathrm{~Hz}\right), 52.43\left(\mathrm{~d},{ }^{2} J_{\mathrm{C}-\mathrm{P}}=5.2 \mathrm{~Hz}\right.$, $\left.\{\mathrm{PO}\} \mathrm{OCH}_{3} \times 2\right) ;{ }^{31} \mathrm{P} \mathrm{NMR}\left(162 \mathrm{MHz}, \mathrm{CDCl}_{3}\right) \delta 27.31$; HRMS for $\mathrm{C}_{24} \mathrm{H}_{21} \mathrm{O} 4 \mathrm{P}$ : calcd. $\left(\mathrm{MH}^{+}\right)$: 405.1250, found: 405.1246

\section{Dimethyl (1-hydroxy-3-phenyl-4-(4-(trimethylsilyl)phenyl)naphthalen-2-yl)phosphonate (3zb)}

White solid; isolated yield $16 \%$ (8 mg). $R_{f} 0.50$ (10\% EtOAc/hexane); Mp 184-186 ${ }^{\circ} \mathrm{C} ;{ }^{1} \mathrm{H}$ $\operatorname{NMR}\left(400 \mathrm{MHz}, \mathrm{CDCl}_{3}\right) \delta 12.40(\mathrm{~d}, J=1.2 \mathrm{~Hz}, 1 \mathrm{H}), 8.45-8.47(\mathrm{~m}, 1 \mathrm{H}), 7.40-7.48(\mathrm{~m}, 2 \mathrm{H})$, 7.29-7.32 (m, 1H), 7.23 (br d, $J=8.0 \mathrm{~Hz}, 2 \mathrm{H}$ ), 6.99-7.02 (m, 3H), 6.94-6.97 (m, 2H), 6.92 (br d, $J=8.0 \mathrm{~Hz}, 2 \mathrm{H}), 3.43\left(\mathrm{~d},{ }^{3} J_{\mathrm{H}-\mathrm{P}}=11.5 \mathrm{~Hz}, 6 \mathrm{H}\right), 0.15(\mathrm{~s}, 9 \mathrm{H}) ;{ }^{13} \mathrm{C}$ NMR $(100 \mathrm{MHz}$, $\left.\mathrm{CDCl}_{3}\right) \delta 162.13\left(\mathrm{~d},{ }^{2} J_{\mathrm{C}-\mathrm{P}}=7.7 \mathrm{~Hz}, \mathrm{C}-\mathrm{OH}\right), 139.38\left(\mathrm{~d}, J_{\mathrm{C}-\mathrm{P}}=4.0 \mathrm{~Hz}\right), 139.01,138.59\left(\mathrm{~d}, J_{\mathrm{C}-\mathrm{P}}\right.$ $=7.3 \mathrm{~Hz}), 137.98,136.08\left(\mathrm{~d}, J_{\mathrm{C}-\mathrm{P}}=1.9 \mathrm{~Hz}\right), 132.36,131.47\left(\mathrm{~d}, J_{\mathrm{C}-\mathrm{P}}=12.6 \mathrm{~Hz}\right), 130.72$, $130.64,129.51,126.72,126.56,126.31,125.68,124.41\left(\mathrm{~d}, J_{\mathrm{C}-\mathrm{P}}=14.2 \mathrm{~Hz}\right), 123.85,99.11(\mathrm{~d}$, $\left.{ }^{1} J_{\mathrm{C}-\mathrm{P}}=180.7 \mathrm{~Hz}\right), 52.42\left(\mathrm{~d},{ }^{2} J_{\mathrm{C}-\mathrm{P}}=5.3 \mathrm{~Hz},\{\mathrm{PO}\} \mathrm{OCH}_{3} \times 2\right),-1.11 ;{ }^{31} \mathrm{P} \mathrm{NMR}(162 \mathrm{MHz}$, $\left.\mathrm{CDCl}_{3}\right) \delta$ 27.38; HRMS for $\mathrm{C}_{27} \mathrm{H}_{29} \mathrm{O}_{4} \mathrm{PSi}$ : calcd. $\left(\mathrm{MH}^{+}\right)$: 477.1645 , found: 477.1643

\section{Dimethyl (1-hydroxy-4-phenyl-3-(4-(trimethylsilyl)phenyl)naphthalen-2-yl)phosphonate (3zb')}

White solid; isolated yield $22 \%(11 \mathrm{mg}) . R_{f} 0.50$ (10\% EtOAc/hexane); Mp $124-126{ }^{\circ} \mathrm{C} ;{ }^{1} \mathrm{H}$ NMR (400 MHz, $\left.\mathrm{CDCl}_{3}\right) \delta 12.40(\mathrm{~d}, J=1.2 \mathrm{~Hz}, 1 \mathrm{H}), 8.44-8.46(\mathrm{~m}, 1 \mathrm{H}), 7.40-7.48(\mathrm{~m}, 2 \mathrm{H})$, 7.29-7.31 (m, 1H), 7.14 (br d, $J=8.0 \mathrm{~Hz}, 2 \mathrm{H}$ ), 7.01-7.10 (m, 3H), 6.91-6.94 (m, 4H), 3.39 $\left(\mathrm{d},{ }^{3} J_{\mathrm{H}-\mathrm{P}}=11.5 \mathrm{~Hz}, 6 \mathrm{H}\right), 0.11(\mathrm{~s}, 9 \mathrm{H}) ;{ }^{13} \mathrm{C} \mathrm{NMR}\left(100 \mathrm{MHz}, \mathrm{CDCl}_{3}\right) \delta 162.09\left(\mathrm{~d},{ }^{2} J_{\mathrm{C}-\mathrm{P}}=7.7\right.$ $\mathrm{Hz}, \mathrm{C}-\mathrm{OH}), 139.81\left(\mathrm{~d}, J_{\mathrm{C}-\mathrm{P}}=4.0 \mathrm{~Hz}\right), 138.72\left(\mathrm{~d}, J_{\mathrm{C}-\mathrm{P}}=7.3 \mathrm{~Hz}\right), 138.61,138.30,136.08(\mathrm{~d}$, $\left.J_{\mathrm{C}-\mathrm{P}}=1.9 \mathrm{~Hz}\right), 131.42,131.33,131.15,129.96,129.50,127.42,126.62,126.19,125.65$, $124.39\left(\mathrm{~d}, J_{\mathrm{C}-\mathrm{P}}=14.2 \mathrm{~Hz}\right), 123.86,99.16\left(\mathrm{~d},{ }^{1} J_{\mathrm{C}-\mathrm{P}}=181.2 \mathrm{~Hz}\right), 52.45\left(\mathrm{~d},{ }^{2} J_{\mathrm{C}-\mathrm{P}}=5.6 \mathrm{~Hz}\right.$, $\left.\{\mathrm{PO}\} \mathrm{OCH}_{3} \times 2\right),-1.16 ;{ }^{31} \mathrm{P} \mathrm{NMR}\left(162 \mathrm{MHz}, \mathrm{CDCl}_{3}\right) \delta 27.05 ; \mathrm{HRMS}$ for $\mathrm{C}_{27} \mathrm{H}_{29} \mathrm{O}_{4} \mathrm{PSi}$ : calcd. $\left(\mathrm{MH}^{+}\right)$: 477.1645, found: 477.1644

Selected X-Ray Crystallographic data for 3zb', $\mathrm{C}_{27} \mathrm{H}_{29} \mathrm{O}_{4} \mathrm{PSi}, M=476.56$, Monoclinic, $P$

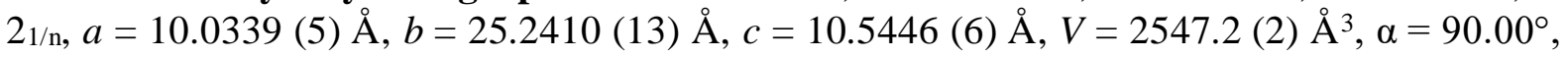
$\beta=107.484(2)^{\circ}, \gamma=90.00^{\circ}, Z=4, D_{c}=1.243 \mathrm{~g} \mathrm{~cm}^{-3}, \mu(\mathrm{Mo}-\mathrm{K} \alpha)=0.185 \mathrm{~mm}^{-1}, F(000)=$ 1008, Reflections Collected: unique 39317/6322 [R(int $)=0.0710]$. Final R indices $[I>2 \mathrm{~s}$ $(I)], R 1=0.0481, \mathrm{wR}_{2}=0.1522$.

Dimethyl (1-hydroxy-4-(4-isopropylphenyl)-3-phenylnaphthalen-2-yl)phosphonate (3zc) White solid; isolated yield 19\% (9 mg). $R_{f} 0.50$ (10\% EtOAc/hexane); Mp 185-188 ${ }^{\circ} \mathrm{C} ;{ }^{1} \mathrm{H}$ NMR $\left(400 \mathrm{MHz}, \mathrm{CDCl}_{3}\right) \delta 12.37(\mathrm{~d}, J=1.2 \mathrm{~Hz}, 1 \mathrm{H}), 8.44-8.46(\mathrm{~m}, 1 \mathrm{H}), 7.41-7.48(\mathrm{~m}, 2 \mathrm{H})$, 7.33-7.35 (m, 1H), 6.99-7.03 (m, 3H), 6.92-6.95 (m, 4H), 6.82-6.85 (m, 2H), $3.42\left(\mathrm{~d},{ }^{3} J_{\mathrm{H}-\mathrm{P}}=\right.$ $11.6 \mathrm{~Hz}, 6 \mathrm{H}), 2.68-2.79(\mathrm{~m}, 1 \mathrm{H}), 1.10(\mathrm{~d}, J=6.9 \mathrm{~Hz}, 6 \mathrm{H}) ;{ }^{13} \mathrm{C} \mathrm{NMR}\left(100 \mathrm{MHz}, \mathrm{CDCl}_{3}\right) \delta$ $162.01\left(\mathrm{~d},{ }^{2} J_{\mathrm{C}-\mathrm{P}}=7.6 \mathrm{~Hz}, \mathrm{C}-\mathrm{OH}\right), 146.70,139.55\left(\mathrm{~d}, J_{\mathrm{C}-\mathrm{P}}=4.1 \mathrm{~Hz}\right), 138.70\left(\mathrm{~d}, J_{\mathrm{C}-\mathrm{P}}=7.2 \mathrm{~Hz}\right)$, 
$136.27\left(\mathrm{~d}, J_{\mathrm{C}-\mathrm{P}}=1.9 \mathrm{~Hz}\right), 135.82,131.57\left(\mathrm{~d}, J_{\mathrm{C}-\mathrm{P}}=12.6 \mathrm{~Hz}\right), 131.27,130.66,129.44,126.79$, $126.46,126.24,125.62,125.40,124.40\left(\mathrm{~d}, J_{\mathrm{C}-\mathrm{P}}=14.3 \mathrm{~Hz}\right), 123.81,99.07\left(\mathrm{~d},{ }^{1} J_{\mathrm{C}-\mathrm{P}}=180.5\right.$ $\mathrm{Hz}), 52.40\left(\mathrm{~d},{ }^{2} J_{\mathrm{C}-\mathrm{P}}=5.3 \mathrm{~Hz},\{\mathrm{PO}\} \mathrm{OCH}_{3} \times 2\right), 33.63,23.89 ;{ }^{31} \mathrm{P} \mathrm{NMR}\left(162 \mathrm{MHz}, \mathrm{CDCl}_{3}\right) \delta$ 27.44; HRMS for $\mathrm{C}_{27} \mathrm{H}_{27} \mathrm{O} 4 \mathrm{P}$ : calcd. $\left(\mathrm{MH}^{+}\right)$: 447.1720, found: 447.1718

\section{Dimethyl (1-hydroxy-3-(4-isopropylphenyl)-4-phenylnaphthalen-2-yl)phosphonate (3zc')}

White solid; isolated yield 23\% (11 mg). $R_{f} 0.50$ (10\% EtOAc/hexane); Mp 151-153 ${ }^{\circ} \mathrm{C} ;{ }^{1} \mathrm{H}$ NMR (400 MHz, $\left.\mathrm{CDCl}_{3}\right) \delta 12.40(\mathrm{~d}, J=1.4 \mathrm{~Hz}, 1 \mathrm{H}), 8.44-8.46(\mathrm{~m}, 1 \mathrm{H}), 7.39-7.47(\mathrm{~m}, 2 \mathrm{H})$, 7.29-7.31 (m, 1H), 7.01-7.10 (m, 3H), 6.91-6.94 (m, 2H), $6.85(\mathrm{br} \mathrm{s}, 4 \mathrm{H}), 3.40\left(\mathrm{~d},{ }^{3} J_{\mathrm{H}-\mathrm{P}}=11.5\right.$ $\mathrm{Hz}, 6 \mathrm{H}), 2.66-2.76(\mathrm{~m}, 1 \mathrm{H}), 1.07(\mathrm{~d}, J=6.9 \mathrm{~Hz}, 6 \mathrm{H}) ;{ }^{13} \mathrm{C} \mathrm{NMR}\left(100 \mathrm{MHz}, \mathrm{CDCl}_{3}\right) \delta 162.01$ $\left(\mathrm{d},{ }^{2} J_{\mathrm{C}-\mathrm{P}}=7.6 \mathrm{~Hz}, \mathrm{C}-\mathrm{OH}\right), 146.70,139.55\left(\mathrm{~d}, J_{\mathrm{C}-\mathrm{P}}=4.1 \mathrm{~Hz}\right), 138.70\left(\mathrm{~d}, J_{\mathrm{C}-\mathrm{P}}=7.2 \mathrm{~Hz}\right), 136.27$ $\left(\mathrm{d}, J_{\mathrm{C}-\mathrm{P}}=1.9 \mathrm{~Hz}\right), 135.82,131.57\left(\mathrm{~d}, J_{\mathrm{C}-\mathrm{P}}=12.6 \mathrm{~Hz}\right), 131.27,130.66,129.44,126.79,126.46$, $126.24,125.62,125.40,124.40\left(\mathrm{~d}, J_{\mathrm{C}-\mathrm{P}}=14.3 \mathrm{~Hz}\right), 123.81,99.07\left(\mathrm{~d},{ }^{1} J_{\mathrm{C}-\mathrm{P}}=180.5 \mathrm{~Hz}\right), 52.40$ $\left(\mathrm{d},{ }^{2} J_{\mathrm{C}-\mathrm{P}}=5.3 \mathrm{~Hz},\{\mathrm{PO}\} \mathrm{OCH}_{3} \mathrm{x} 2\right), 33.63,23.89 ;{ }^{31} \mathrm{P} \mathrm{NMR}\left(162 \mathrm{MHz}, \mathrm{CDCl}_{3}\right) \delta 27.17$; HRMS for $\mathrm{C}_{27} \mathrm{H}_{27} \mathrm{O}_{4} \mathrm{P}$ : calcd. $\left(\mathrm{MH}^{+}\right)$: 447.1720 , found: 447.1719

\section{4-Phenyl-2-(phenylsulfonyl)naphthalen-1-ol (3zd)}

Brown viscous liquid; isolated yield $54 \%(20 \mathrm{mg}) . R_{f} 0.50\left(5 \%\right.$ EtOAc/hexane); ${ }^{1} \mathrm{H}$ NMR $\left(300 \mathrm{MHz}, \mathrm{CDCl}_{3}\right) \delta 10.35(\mathrm{~s}, 1 \mathrm{H}), 8.43-8.48(\mathrm{~m}, 1 \mathrm{H}), 7.93-7.97(\mathrm{~m}, 2 \mathrm{H}), 7.73-7.77(\mathrm{~m}, 1 \mathrm{H})$, 7.53-7.56 (m, 3H), 7.45-7.51 (m, 2H), 7.37-7.42 (m, 4H), 7.31-7.35 (m, 2H); ${ }^{13} \mathrm{C}$ NMR (75 $\left.\mathrm{MHz}_{\mathrm{CDCl}}\right) \delta 153.94,141.85,139.06,135.40,133.67,133.31,130.08,129.97,129.48$, $128.42,127.62,126.89,126.53,126.07,125.33,124.18,123.11,115.29$; HRMS for $\mathrm{C}_{22} \mathrm{H}_{16} \mathrm{O}_{3} \mathrm{~S}$ : calcd. $\left(\mathrm{MH}^{+}\right)$: 361.0893, found: 361.0895

\section{4-(4-Ethylphenyl)-2-(phenylsulfonyl)naphthalen-1-ol (3ze)}

Brown viscous liquid; isolated yield $60 \%$ (24 mg). $R_{f} 0.50$ (5\% EtOAc/hexane); ${ }^{1} \mathrm{H}$ NMR $\left(300 \mathrm{MHz}, \mathrm{CDCl}_{3}\right) \delta 10.33(\mathrm{~s}, 1 \mathrm{H}), 8.41-8.46(\mathrm{~m}, 1 \mathrm{H}), 7.92-7.96(\mathrm{~m}, 2 \mathrm{H}), 7.75-7.80(\mathrm{~m}, 1 \mathrm{H})$, 7.50-7.54 (m, 3H), 7.45-7.48 (m, 2H), $7.39(\mathrm{~s}, 1 \mathrm{H}), 7.24$ (br s, 4H), $2.68(\mathrm{q}, J=7.6 \mathrm{~Hz}, 2 \mathrm{H})$, $1.26(\mathrm{t}, J=7.6 \mathrm{~Hz}, 3 \mathrm{H}) ;{ }^{13} \mathrm{C}$ NMR $\left(75 \mathrm{MHz}, \mathrm{CDCl}_{3}\right) \delta 153.78,143.72,141.87,136.34$, $135.51,133.63,133.33,130.01,129.86,129.45,127.90,126.90,126.46,126.17,125.33$, 124.14, 123.04, 115.27, 28.60, 15.53; HRMS for $\mathrm{C}_{24} \mathrm{H}_{20} \mathrm{O} 3 \mathrm{~S}$ : calcd. $\left(\mathrm{MH}^{+}\right)$: 389.1206, found: 389.1203

\section{4-(4-(tert-butyl)phenyl)-2-(phenylsulfonyl)naphthalen-1-ol (3zf)}

Brown viscous liquid; isolated yield 63\% (26 mg). $R_{f} 0.50$ (5\% EtOAc/hexane); ${ }^{1} \mathrm{H}$ NMR $\left(300 \mathrm{MHz}, \mathrm{CDCl}_{3}\right) \delta 10.33(\mathrm{~s}, 1 \mathrm{H}), 8.40-8.45(\mathrm{~m}, 1 \mathrm{H}), 7.91-7.95(\mathrm{~m}, 2 \mathrm{H}), 7.77-7.81(\mathrm{~m}, 1 \mathrm{H})$, 7.51-7.54 (m, 2H), 7.47-7.50 (m, 2H), 7.42-7.45 (m, 2H), 7.39-7.42 (m, 2H), 7.23-7.28 (m, $2 \mathrm{H}), 1.33(\mathrm{~s}, 9 \mathrm{H}) ;{ }^{13} \mathrm{C}$ NMR $\left(75 \mathrm{MHz}, \mathrm{CDCl}_{3}\right) \delta 153.82,150.64,141.91,136.11,135.54$, 133.67, 133.31, 129.89, 129.77, 129.48, 126.94, 126.50, 126.26, 125.37, 124.17, 123.11, 115.32, 34.68, 31.44; HRMS for $\mathrm{C}_{26} \mathrm{H}_{24} \mathrm{O}_{3} \mathrm{~S}$ : calcd. $\left(\mathrm{MH}^{+}\right)$: 417.1519 , found: 417.1519

\section{4-(4-fluorophenyl)-2-(phenylsulfonyl)naphthalen-1-ol (3zg)}

Brown viscous liquid; isolated yield $56 \%(21 \mathrm{mg}) . R_{f} 0.50\left(5 \%\right.$ EtOAc/hexane); ${ }^{1} \mathrm{H}$ NMR $\left(300 \mathrm{MHz}, \mathrm{CDCl}_{3}\right) \delta 10.34(\mathrm{~s}, 1 \mathrm{H}), 8.42-8.47(\mathrm{~m}, 1 \mathrm{H}), 7.92-7.97(\mathrm{~m}, 2 \mathrm{H}), 7.65-7.70(\mathrm{~m}, 1 \mathrm{H})$, 7.50-7.57 (m, 3H), 7.44-7.48 (m, 2H), $7.37(\mathrm{~s}, 1 \mathrm{H}), 7.25-7.31(\mathrm{~m}, 2 \mathrm{H}), 7.05-7.13(\mathrm{~m}, 2 \mathrm{H})$; ${ }^{13} \mathrm{C}$ NMR $\left(75 \mathrm{MHz}, \mathrm{CDCl}_{3}\right) \delta 162.46\left(\mathrm{~d}, J_{\mathrm{C}-\mathrm{F}}=245.3 \mathrm{~Hz}\right), 154.09,141.83,135.46,134.99(\mathrm{~d}$, $J=3.4 \mathrm{~Hz}), 133.76,132.24,131.68(\mathrm{~d}, J=7.9 \mathrm{~Hz}), 130.14,129.54,126.91,126.66,125.86$, 125.38, 124.30, 123.23, $115.43(\mathrm{~d}, J=21.3 \mathrm{~Hz}), 115.33$; HRMS for $\mathrm{C}_{22} \mathrm{H}_{15} \mathrm{FO}_{3} \mathrm{~S}$ : calcd. $\left(\mathrm{MH}^{+}\right)$: 379.0799, found: 379.0806 


\section{Ethyl 1-hydroxy-4-phenyl-2-naphthoate (3zh)}

Light yellow solid; isolated yield 64\% (19 mg). $R_{f} 0.50$ (2\% EtOAc/hexane); Mp 112-114 ${ }^{\circ} \mathrm{C}$; ${ }^{1} \mathrm{H}$ NMR $\left(300 \mathrm{MHz}, \mathrm{CDCl}_{3}\right) \delta 12.04(\mathrm{~s}, 1 \mathrm{H}), 8.45-8.48(\mathrm{~m}, 1 \mathrm{H}), 7.75-7.79(\mathrm{~m}, 1 \mathrm{H}), 7.70(\mathrm{~s}$, $1 \mathrm{H}), 7.50-7.54(\mathrm{~m}, 2 \mathrm{H}), 7.38-7.48(\mathrm{~m}, 5 \mathrm{H}), 4.42(\mathrm{q}, J=7.1 \mathrm{~Hz}, 2 \mathrm{H}), 1.39(\mathrm{t}, J=7.1 \mathrm{~Hz}, 3 \mathrm{H})$; ${ }^{13} \mathrm{C}$ NMR $\left(75 \mathrm{MHz}, \mathrm{CDCl}_{3}\right) \delta 171.32,160.59,140.43,135.71,131.37,130.41,129.61$, $128.56,127.41,126.09,125.86,125.21,125.12,124.33,105.60,61.70,14.49$; HRMS for $\mathrm{C}_{19} \mathrm{H}_{16} \mathrm{O}_{3}$ : calcd. $\left(\mathrm{MH}^{+}\right)$: 293.1172, found: 293.1175

\section{Ethyl 1-hydroxy-4-(4-methoxyphenyl)-2-naphthoate (3zi)}

Light yellow solid; isolated yield 66\% (21 mg). $R_{f} 0.50$ (2\% EtOAc/hexane); Mp 136-138 ${ }^{\circ} \mathrm{C}$; ${ }^{1} \mathrm{H}$ NMR $\left(300 \mathrm{MHz}, \mathrm{CDCl}_{3}\right) \delta 11.96(\mathrm{~s}, 1 \mathrm{H}), 8.38-8.43(\mathrm{~m}, 1 \mathrm{H}), 7.70-7.75(\mathrm{~m}, 1 \mathrm{H}), 7.62(\mathrm{~s}$, $1 \mathrm{H}), 7.41-7.48(\mathrm{~m}, 2 \mathrm{H}), 7.26-7.31(\mathrm{~m}, 2 \mathrm{H}), 6.91-6.95(\mathrm{~m}, 2 \mathrm{H}), 4.36(\mathrm{q}, J=7.1 \mathrm{~Hz}, 2 \mathrm{H}), 3.80$ $(\mathrm{s}, 3 \mathrm{H}), 1.33(\mathrm{t}, J=7.1 \mathrm{~Hz}, 3 \mathrm{H}) ;{ }^{13} \mathrm{C}$ NMR $\left(75 \mathrm{MHz}, \mathrm{CDCl}_{3}\right) \delta 171.30,160.37,159.12$, $135.95,132.74,131.40,130.98,129.50,126.11,125.77,125.20,125.00,124.29$, 113.99, 105.58, 61.64, 55.56, 14.46; HRMS for $\mathrm{C}_{20} \mathrm{H}_{18} \mathrm{O}_{4}$ : calcd. $\left(\mathrm{MH}^{+}\right)$: 323.1278 , found: 323.1277

\section{Ethyl 4-(4-fluorophenyl)-1-hydroxy-2-naphthoate (3zj)}

Light yellow solid; isolated yield 62\% (19 mg). $R_{f} 0.50$ (2\% EtOAc/hexane); Mp 94-96 ${ }^{\circ} \mathrm{C}$; ${ }^{1} \mathrm{H}$ NMR $\left(300 \mathrm{MHz}, \mathrm{CDCl}_{3}\right) \delta 12.00(\mathrm{~s}, 1 \mathrm{H}), 8.40-8.45(\mathrm{~m}, 1 \mathrm{H}), 7.64-7.68(\mathrm{~m}, 1 \mathrm{H}), 7.62(\mathrm{~s}$, $1 \mathrm{H}), 7.43-7.52(\mathrm{~m}, 2 \mathrm{H}), 7.30-7.37(\mathrm{~m}, 2 \mathrm{H}), 7.05-7.13(\mathrm{~m}, 2 \mathrm{H}), 4.38(\mathrm{q}, J=7.1 \mathrm{~Hz}, 2 \mathrm{H}), 1.36$ $(\mathrm{t}, J=7.1 \mathrm{~Hz}, 3 \mathrm{H}) ;{ }^{13} \mathrm{C} \mathrm{NMR}\left(75 \mathrm{MHz}, \mathrm{CDCl}_{3}\right) \delta 171.22,162.45\left(\mathrm{~d}, J_{\mathrm{C}-\mathrm{F}}=244.6 \mathrm{~Hz}\right)$, $160.70,136.31(\mathrm{~d}, J=3.2 \mathrm{~Hz}), 135.72,131.92(J=7.9 \mathrm{~Hz}), 130.23,129.73,125.89(\mathrm{~d}, J=$ $7.4 \mathrm{~Hz}), 125.23,125.20,124.42,115.46(\mathrm{~d}, J=21.2 \mathrm{~Hz}), 105.56,61.75,14.49$; HRMS for $\mathrm{C}_{19} \mathrm{H}_{15} \mathrm{FO}_{3}$ : calcd. $\left(\mathrm{MH}^{+}\right)$: 311.078 , found: 311.080

\section{Ethyl 1-hydroxy-6,7-dimethoxy-4-phenyl-2-naphthoate (3zk)}

Light yellow solid; isolated yield 36\% (13 mg). $R_{f} 0.50$ (2\% EtOAc/hexane); Mp 151-153 ${ }^{\circ} \mathrm{C}$; ${ }^{1} \mathrm{H}$ NMR $\left(300 \mathrm{MHz}, \mathrm{CDCl}_{3}\right) \delta 11.92(\mathrm{~s}, 1 \mathrm{H}), 7.67(\mathrm{~s}, 1 \mathrm{H}), 7.54(\mathrm{~s}, 1 \mathrm{H}), 7.38-7.44(\mathrm{~m}, 4 \mathrm{H})$, 7.30-7.37 (m, 1H), $7.05(\mathrm{~s}, 1 \mathrm{H}), 4.36(\mathrm{q}, J=7.1 \mathrm{~Hz}, 2 \mathrm{H}), 3.97(\mathrm{~s}, 3 \mathrm{H}), 3.74(\mathrm{~s}, 3 \mathrm{H}), 1.34(\mathrm{t}, J$ $=7.1 \mathrm{~Hz}, 3 \mathrm{H}) ;{ }^{13} \mathrm{C} \mathrm{NMR}\left(75 \mathrm{MHz}, \mathrm{CDCl}_{3}\right) \delta 171.45,159.17,152.13,149.20,140.80,132.00$, $130.15,130.11,128.64,127.34,123.95,120.03,105.31,104.79,103.04,61.49,56.22,55.94$, 14.48; HRMS for $\mathrm{C}_{21} \mathrm{H}_{20} \mathrm{O}_{5}$ : calcd. $\left(\mathrm{MH}^{+}\right)$: 353.1384, found: 353.1381

\section{Ethyl 1-hydroxy-5,6-dimethoxy-4-phenyl-2-naphthoate (3zk')}

Light yellow solid; isolated yield 23\% (8 mg). $R_{f} 0.50$ (2\% EtOAc/hexane); Mp 101-103 ${ }^{\circ} \mathrm{C}$; ${ }^{1} \mathrm{H}$ NMR $\left(300 \mathrm{MHz}, \mathrm{CDCl}_{3}\right) \delta 12.02(\mathrm{~s}, 1 \mathrm{H}), 8.25(\mathrm{~d}, J=9.2 \mathrm{~Hz}, 1 \mathrm{H}), 7.48(\mathrm{~s}, 1 \mathrm{H}), 7.23-7.32$ $(\mathrm{m}, 6 \mathrm{H}), 4.35(\mathrm{q}, J=7.1 \mathrm{~Hz}, 2 \mathrm{H}), 3.90(\mathrm{~s}, 3 \mathrm{H}), 3.05(\mathrm{~s}, 3 \mathrm{H}), 1.32(\mathrm{t}, J=7.1 \mathrm{~Hz}, 3 \mathrm{H}) ;{ }^{13} \mathrm{C}$ NMR $\left(75 \mathrm{MHz}, \mathrm{CDCl}_{3}\right) \delta 171.06,160.64,153.31,143.95,143.79,130.90,129.48,128.39$, $127.75,126.83,125.96,121.23,121.00,113.13,103.45,61.37,60.39,56.32,14.31$; HRMS for $\mathrm{C}_{21} \mathrm{H}_{20} \mathrm{O}_{5}$ : calcd. $\left(\mathrm{MH}^{+}\right)$: 353.1384 , found: 353.1383

\section{Dimethyl (10-hydroxyphenanthren-9-yl)phosphonate (5a)}

Brown viscous liquid; isolated yield $76 \%$ (23 mg). $R_{f} 0.50$ (10\% EtOAc/hexane); ${ }^{1} \mathrm{H}$ NMR $\left(400 \mathrm{MHz} \mathrm{CDCl}_{3}\right) \delta 12.54(\mathrm{~s}, 1 \mathrm{H}), 8.52-8.56(\mathrm{~m}, 2 \mathrm{H}), 8.49(\mathrm{~d}, J=8.2 \mathrm{~Hz}, 1 \mathrm{H}), 8.02(\mathrm{~d}, J=$ $8.2 \mathrm{~Hz}, 1 \mathrm{H}), 7.70-7.74(\mathrm{~m}, 1 \mathrm{H}), 7.58-7.62(\mathrm{~m}, 1 \mathrm{H}), 7.48-7.52(\mathrm{~m}, 1 \mathrm{H}), 7.41-7.45(\mathrm{~m}, 1 \mathrm{H})$, $3.72\left(\mathrm{~d},{ }^{3} J_{\mathrm{H}-\mathrm{P}}=11.8 \mathrm{~Hz}, 6 \mathrm{H}\right) ;{ }^{13} \mathrm{C}$ NMR $\left(100 \mathrm{MHz}, \mathrm{CDCl}_{3}\right) \delta 163.32\left(\mathrm{~d},{ }^{2} J_{\mathrm{C}-\mathrm{P}}=6.9 \mathrm{~Hz}, \mathrm{C}-\right.$ $\mathrm{OH}), 133.86\left(\mathrm{~d}, J_{\mathrm{C}-\mathrm{P}}=2.0 \mathrm{~Hz}\right), 130.48\left(\mathrm{~d}, J_{\mathrm{C}-\mathrm{P}}=7.8 \mathrm{~Hz}\right), 130.27,127.79,127.00\left(\mathrm{~d}, J_{\mathrm{C}-\mathrm{P}}=\right.$ $0.9 \mathrm{~Hz}), 125.92\left(\mathrm{~d}, J_{\mathrm{C}-\mathrm{P}}=11.3 \mathrm{~Hz}\right), 125.43\left(\mathrm{~d}, J_{\mathrm{C}-\mathrm{P}}=14.5 \mathrm{~Hz}\right), 125.06\left(\mathrm{~d}, J_{\mathrm{C}-\mathrm{P}}=4.3 \mathrm{~Hz}\right)$, $124.83,124.33,122.94,122.43,92.96\left(\mathrm{~d},{ }^{1} J_{\mathrm{C}-\mathrm{P}}=175.4 \mathrm{~Hz}\right), 52.79\left(\mathrm{~d},{ }^{2} J_{\mathrm{C}-\mathrm{P}}=4.1 \mathrm{~Hz}\right.$, 
$\{\mathrm{PO}\} \mathrm{OCH}_{3} \times 2$ ); ${ }^{31} \mathrm{P}$ NMR $\left(161.9 \mathrm{MHz}, \mathrm{CDCl}_{3}\right) \delta 30.02$; HRMS for $\mathrm{C}_{16} \mathrm{H}_{15} \mathrm{O}_{4} \mathrm{P}$ : calcd. $\left(\mathrm{MH}^{+}\right)$: 303.0781, found: 303.0782

\section{Dimethyl (10-hydroxy-7-methylphenanthren-9-yl)phosphonate (5b)}

Brown viscous liquid; isolated yield $78 \%(25 \mathrm{mg}) . R_{f} 0.50(10 \% \mathrm{EtOAc} / \mathrm{hexane}) ;{ }^{1} \mathrm{H}$ NMR $\left(300 \mathrm{MHz}, \mathrm{CDCl}_{3}\right) \delta 12.50(\mathrm{~d}, J=1.0 \mathrm{~Hz}, 1 \mathrm{H}), 8.49(\mathrm{~d}, J=8.3 \mathrm{~Hz}, 1 \mathrm{H}), 8.44(\mathrm{dd}, J=8.3 \mathrm{~Hz}$, $1.1 \mathrm{~Hz}, 1 \mathrm{H}), 8.40(\mathrm{dd}, J=8.4 \mathrm{~Hz}, 1.9 \mathrm{~Hz}, 1 \mathrm{H}), 7.77(\mathrm{~s}, 1 \mathrm{H}), 7.66-7.71(\mathrm{~m}, 1 \mathrm{H}), 7.52-7.57(\mathrm{~m}$, $1 \mathrm{H}), 7.05(\mathrm{dd}, J=8.5 \mathrm{~Hz}, 1.4 \mathrm{~Hz}, 1 \mathrm{H}), 3.70\left(\mathrm{~d},{ }^{3} J_{\mathrm{H}-\mathrm{P}}=11.8 \mathrm{~Hz}, 6 \mathrm{H}\right), 2.46(\mathrm{~s}, 3 \mathrm{H}) ;{ }^{13} \mathrm{C} \mathrm{NMR}$ $\left(100 \mathrm{MHz} \mathrm{CDCl}_{3}\right) \delta 163.43\left(\mathrm{~d},{ }^{2} J_{\mathrm{C}-\mathrm{P}}=6.8 \mathrm{~Hz}, \mathrm{C}-\mathrm{OH}\right), 137.67,133.94,130.64\left(\mathrm{~d}, J_{\mathrm{C}-\mathrm{P}}=7.7\right.$ $\mathrm{Hz}), 130.23,126.55,125.99,125.05\left(\mathrm{~d}, J_{\mathrm{C}-\mathrm{P}}=14.4 \mathrm{~Hz}\right), 124.86,124.81,123.75\left(\mathrm{~d}, J_{\mathrm{C}-\mathrm{P}}=\right.$ $11.5 \mathrm{~Hz}), 122.83,122.23,92.66\left(\mathrm{~d},{ }^{1} J_{\mathrm{C}-\mathrm{P}}=175.1 \mathrm{~Hz}\right), 52.81\left(\mathrm{~d},{ }^{2} J_{\mathrm{C}-\mathrm{P}}=3.9 \mathrm{~Hz},\{\mathrm{PO}\} \mathrm{OCH}_{3} \mathrm{X}\right.$ 2), 22.03; ${ }^{31} \mathrm{P}$ NMR (121.5 MHz, $\left.\mathrm{CDCl}_{3}\right) \delta$ 30.35; HRMS for $\mathrm{C}_{17} \mathrm{H}_{17} \mathrm{O} 4 \mathrm{P}$ : calcd. $\left(\mathrm{MH}^{+}\right)$: 317.0937, found: 317.0937

\section{Dimethyl (10-hydroxy-7-methoxyphenanthren-9-yl)phosphonate (5c)}

Brown solid; isolated yield 82\% (27 mg). $R_{f} 0.50$ (10\% EtOAc/hexane); Mp 87-88 ${ }^{\circ} \mathrm{C} ;{ }^{1} \mathrm{H}$ NMR $\left(300 \mathrm{MHz}, \mathrm{CDCl}_{3}\right) \delta 12.49(\mathrm{~d}, J=1.3 \mathrm{~Hz}, 1 \mathrm{H}), 8.39-8.44(\mathrm{~m}, 3 \mathrm{H}), 7.64-7.70(\mathrm{~m}, 1 \mathrm{H})$, 7.48-7.53 (m, 1H), $7.46(\mathrm{~d}, J=2.6 \mathrm{~Hz}, 1 \mathrm{H}), 7.05(\mathrm{dd}, J=9.1 \mathrm{~Hz}, 2.6 \mathrm{~Hz}, 1 \mathrm{H}), 3.86(\mathrm{~s}, 3 \mathrm{H})$, $3.71\left(\mathrm{~d},{ }^{3} J_{\mathrm{H}-\mathrm{P}}=11.8 \mathrm{~Hz}, 6 \mathrm{H}\right) ;{ }^{13} \mathrm{C} \mathrm{NMR}\left(75 \mathrm{MHz}, \mathrm{CDCl}_{3}\right) \delta 164.15\left(\mathrm{~d},{ }^{2} J_{\mathrm{C}-\mathrm{P}}=6.9 \mathrm{~Hz}, \mathrm{C}-\right.$ $\mathrm{OH}), 159.34,134.21\left(\mathrm{~d}, J_{\mathrm{C}-\mathrm{P}}=2.1 \mathrm{~Hz}\right), 132.28\left(\mathrm{~d}, J_{\mathrm{C}-\mathrm{P}}=7.5 \mathrm{~Hz}\right), 130.54,126.13\left(\mathrm{~d}, J_{\mathrm{C}-\mathrm{P}}=\right.$ $0.9 \mathrm{~Hz}), 125.03\left(\mathrm{~d}, J_{\mathrm{C}-\mathrm{P}}=1.4 \mathrm{~Hz}\right), 124.62,124.44,122.12,120.27\left(\mathrm{~d}, J_{\mathrm{C}-\mathrm{P}}=11.2 \mathrm{~Hz}\right), 113.55$, $107.46\left(\mathrm{~d}, J_{\mathrm{C}-\mathrm{P}}=4.4 \mathrm{~Hz}\right), 92.77\left(\mathrm{~d},{ }^{1} J_{\mathrm{C}-\mathrm{P}}=175.3 \mathrm{~Hz}\right), 55.53,52.98\left(\mathrm{~d},{ }^{2} J_{\mathrm{C}-\mathrm{P}}=4.1 \mathrm{~Hz}\right.$, $\left.\{\mathrm{PO}\} \mathrm{OCH}_{3} \times 2\right) ;{ }^{31} \mathrm{P} \mathrm{NMR}\left(121.5 \mathrm{MHz}, \mathrm{CDCl}_{3}\right) \delta 30.13$; HRMS for $\mathrm{C}_{17} \mathrm{H}_{17} \mathrm{O}_{5} \mathrm{P}$ : calcd. $\left(\mathrm{MH}^{+}\right)$: 333.0886, found: 333.0890

\section{Dimethyl (7-chloro-10-hydroxyphenanthren-9-yl)phosphonate (5d)}

Colorless viscous liquid; isolated yield $75 \%(25 \mathrm{mg}) . R_{f} 0.50$ (10\% EtOAc/hexane); ${ }^{1} \mathrm{H}$ NMR $\left(400 \mathrm{MHz}, \mathrm{CDCl}_{3}\right) \delta 12.56(\mathrm{~d}, J=1.3 \mathrm{~Hz}, 1 \mathrm{H}), 8.46(\mathrm{~d}, J=8.4 \mathrm{~Hz}, 2 \mathrm{H}), 8.42(\mathrm{dd}, J=8.8 \mathrm{~Hz}$, $1.9 \mathrm{~Hz}, 1 \mathrm{H}), 7.96(\mathrm{~d}, J=2.0 \mathrm{~Hz}, 1 \mathrm{H}), 7.70-7.74(\mathrm{~m}, 1 \mathrm{H}), 7.58-7.62(\mathrm{~m}, 1 \mathrm{H}), 7.37$ (dd, $J=8.8$ $\mathrm{Hz}, 2.1 \mathrm{~Hz}, 1 \mathrm{H}), 3.73\left(\mathrm{~d},{ }^{3} J_{\mathrm{H}-\mathrm{P}}=11.8 \mathrm{~Hz}, 6 \mathrm{H}\right) ;{ }^{13} \mathrm{C} \mathrm{NMR}\left(100 \mathrm{MHz}, \mathrm{CDCl}_{3}\right) \delta 164.01\left(\mathrm{~d},{ }^{2} J_{\mathrm{C}-}\right.$ $\mathrm{P}=6.8 \mathrm{~Hz}, \mathrm{C}-\mathrm{OH}), 133.84,133.31\left(\mathrm{~d}, J_{\mathrm{C}-\mathrm{P}}=1.9 \mathrm{~Hz}\right), 131.80\left(\mathrm{~d}, J_{\mathrm{C}-\mathrm{P}}=7.4 \mathrm{~Hz}\right), 130.60$, $127.26,125.31\left(\mathrm{~d}, J_{\mathrm{C}-\mathrm{P}}=14.1 \mathrm{~Hz}\right), 124.98\left(\mathrm{~d}, J_{\mathrm{C}-\mathrm{P}}=1.3 \mathrm{~Hz}\right), 124.74,124.40,124.36,124.28$, $122.35,92.55\left(\mathrm{~d},{ }^{1} J_{\mathrm{C}-\mathrm{P}}=177.1 \mathrm{~Hz}\right), 52.92\left(\mathrm{~d},{ }^{2} J_{\mathrm{C}-\mathrm{P}}=4.4 \mathrm{~Hz},\{\mathrm{PO}\} \mathrm{OCH}_{3} \times 2\right) ;{ }^{31} \mathrm{P} \mathrm{NMR}$ $\left(161.9 \mathrm{MHz}, \mathrm{CDCl}_{3}\right) \delta$ 28.92; HRMS for $\mathrm{C}_{16} \mathrm{H}_{14} \mathrm{ClO}_{4} \mathrm{P}$ : calcd. $\left(\mathrm{MH}^{+}\right)$: 337.0391, found: 337.0391

\section{Dimethyl (7-fluoro-10-hydroxyphenanthren-9-yl)phosphonate (5e)}

White solid; isolated yield 78\% (25 mg). $R_{f} 0.50$ (10\% EtOAc/hexane); Mp 97-99 ${ }^{\circ} \mathrm{C} ;{ }^{1} \mathrm{H}$ NMR (300 MHz, $\left.\mathrm{CDCl}_{3}\right) \delta 12.53(\mathrm{~d}, J=1.4 \mathrm{~Hz}, 1 \mathrm{H}), 8.42-8.49(\mathrm{~m}, 3 \mathrm{H}), 7.63-7.73(\mathrm{~m}, 2 \mathrm{H})$, 7.54-7.59 (m, 1H), 7.11-7.17 $(\mathrm{m}, 1 \mathrm{H}), 3.71\left(\mathrm{~d},{ }^{3} J_{\mathrm{H}-\mathrm{P}}=11.7 \mathrm{~Hz}, 6 \mathrm{H}\right) ;{ }^{13} \mathrm{C}$ NMR $(75 \mathrm{MHz}$, $\left.\mathrm{CDCl}_{3}\right) \delta 164.16\left(\mathrm{~d},{ }^{2} J_{\mathrm{C}-\mathrm{P}}=6.6 \mathrm{~Hz}, \mathrm{C}-\mathrm{OH}\right), 162.19\left(\mathrm{~d},{ }^{1} J_{\mathrm{C}-\mathrm{F}}=244.1 \mathrm{~Hz}\right), 133.53,132.24(\mathrm{t}$, $\left.J_{\mathrm{C}-\mathrm{P}}=8.6 \mathrm{~Hz}\right), 130.58,126.78,125.07,124.97,124.85,122.49\left(\mathrm{~d}, J_{\mathrm{C}-\mathrm{P}}=11.2 \mathrm{~Hz}\right), 122.26$, $112.84\left(\mathrm{~d}, J_{\mathrm{C}-\mathrm{F}}=23.2 \mathrm{~Hz}\right), 110.33\left(\mathrm{dd}, J_{\mathrm{C}-\mathrm{F}}=23.6 \mathrm{~Hz}, J_{\mathrm{C}-\mathrm{P}}=4.1 \mathrm{~Hz}\right), 92.84\left(\mathrm{~d},{ }^{1} J_{\mathrm{C}-\mathrm{P}}=176.9\right.$ $\mathrm{Hz}), 52.86\left(\mathrm{~d},{ }^{2} J_{\mathrm{C}-\mathrm{P}}=4.1 \mathrm{~Hz},\{\mathrm{PO}\} \mathrm{OCH}_{3} \times 2\right) ;{ }^{31} \mathrm{P} \mathrm{NMR}\left(121.5 \mathrm{MHz}, \mathrm{CDCl}_{3}\right) \delta 29.02 ;$ HRMS for $\mathrm{C}_{16} \mathrm{H}_{14} \mathrm{FO} \mathrm{O}_{4} \mathrm{P}$ : calcd. $\left(\mathrm{MH}^{+}\right)$: 321.0687 , found: 321.0682

\section{Dimethyl (10-hydroxy-7-(trifluoromethyl)phenanthren-9-yl)phosphonate (5f)}

White solid; isolated yield 69\% (26 mg). $R_{f} 0.50$ (10\% EtOAc/hexane); Mp 118-120 ${ }^{\circ} \mathrm{C} ;{ }^{1} \mathrm{H}$ NMR $\left(300 \mathrm{MHz}, \mathrm{CDCl}_{3}\right) \delta 12.59(\mathrm{~d}, J=0.5 \mathrm{~Hz}, 1 \mathrm{H}), 8.60(\mathrm{~d}, J=8.6 \mathrm{~Hz}, 1 \mathrm{H}), 8.54(\mathrm{~d}, J=$ $8.3 \mathrm{~Hz}, 1 \mathrm{H}), 8.50(\mathrm{dd}, J=8.2 \mathrm{~Hz}, 1.3 \mathrm{~Hz}, 1 \mathrm{H}), 8.26(\mathrm{~s}, 1 \mathrm{H}), 7.73-7.79(\mathrm{~m}, 1 \mathrm{H}), 7.59-7.68(\mathrm{~m}$, $2 \mathrm{H}), 3.74\left(\mathrm{~d},{ }^{3} J_{\mathrm{H}-\mathrm{P}}=11.7 \mathrm{~Hz}, 6 \mathrm{H}\right) ;{ }^{13} \mathrm{C} \mathrm{NMR}\left(100 \mathrm{MHz}, \mathrm{CDCl}_{3}\right) \delta 163.99\left(\mathrm{~d},{ }^{2} J_{\mathrm{C}-\mathrm{P}}=6.7 \mathrm{~Hz}\right.$, 
C-OH $), 132.92\left(\mathrm{~d}, J_{\mathrm{C}-\mathrm{P}}=1.5 \mathrm{~Hz}\right), 130.70,130.27\left(\mathrm{~d}, J_{\mathrm{C}-\mathrm{P}}=7.5 \mathrm{~Hz}\right), 129.42\left(\mathrm{q}, J_{\mathrm{C}-\mathrm{F}}=32.0\right.$ $\mathrm{Hz}), 128.21,128.10,126.13\left(\mathrm{~d}, J_{\mathrm{C}-\mathrm{P}}=14.1 \mathrm{~Hz}\right), 125.05,124.36\left(\mathrm{q},{ }^{1} J_{\mathrm{C}-\mathrm{F}}=270.8 \mathrm{~Hz}\right), 123.67$, 122.83, 122.20-122.37 (m), $121.31\left(\mathrm{q}, J_{\mathrm{C}-\mathrm{F}}=3.3 \mathrm{~Hz}\right), 93.41\left(\mathrm{~d},{ }^{1} J_{\mathrm{C}-\mathrm{P}}=177.9 \mathrm{~Hz}\right), 52.97(\mathrm{~d}$, $\left.{ }^{2} J_{\mathrm{C}-\mathrm{P}}=4.6 \mathrm{~Hz},\{\mathrm{PO}\} \mathrm{OCH}_{3} \times 2\right)$; ${ }^{31} \mathrm{P} \mathrm{NMR}\left(121.5 \mathrm{MHz}, \mathrm{CDCl}_{3}\right) \delta 28.38$; HRMS for $\mathrm{C}_{17} \mathrm{H}_{14} \mathrm{~F}_{3} \mathrm{O}_{4} \mathrm{P}$ : calcd. $\left(\mathrm{MH}^{+}\right)$: 371.0655, found: 371.0653

\section{Dimethyl (6-hydroxychrysen-5-yl)phosphonate (5g)}

Brown solid; isolated yield 70\% (25 mg). $R_{f} 0.50$ (10\% EtOAc/hexane); Mp 132-134 ${ }^{\circ} \mathrm{C} ;{ }^{1} \mathrm{H}$ NMR $\left(300 \mathrm{MHz}, \mathrm{CDCl}_{3}\right) \delta 12.68(\mathrm{~d}, J=1.3 \mathrm{~Hz}, 1 \mathrm{H}), 9.01($ br s, $1 \mathrm{H}), 8.70(\mathrm{~d}, J=8.2 \mathrm{~Hz}$, $1 \mathrm{H}), 8.46(\mathrm{~d}, J=8.1 \mathrm{~Hz}, 1 \mathrm{H}), 8.42(\mathrm{~s}, 1 \mathrm{H}), 7.91-7.99(\mathrm{~m}, 2 \mathrm{H}), 7.72-7.78(\mathrm{~m}, 1 \mathrm{H}), 7.58-7.63$ $(\mathrm{m}, 1 \mathrm{H}), 7.41-7.49(\mathrm{~m}, 2 \mathrm{H}), 3.73\left(\mathrm{~d},{ }^{3} J_{\mathrm{H}-\mathrm{P}}=11.9 \mathrm{~Hz}, 6 \mathrm{H}\right) ;{ }^{13} \mathrm{C} \mathrm{NMR}\left(125 \mathrm{MHz}, \mathrm{CDCl}_{3}\right) \delta$ $163.97\left(\mathrm{~d},{ }^{2} J_{\mathrm{C}-\mathrm{P}}=6.4 \mathrm{~Hz}, \mathrm{C}-\mathrm{OH}\right), 133.97,132.71,130.56,130.23,128.38\left(\mathrm{~d}, J_{\mathrm{C}-\mathrm{P}}=7.8 \mathrm{~Hz}\right)$, $128.10,127.79,127.45,126.28,126.03\left(\mathrm{~d}, J_{\mathrm{C}-\mathrm{P}}=14.4 \mathrm{~Hz}\right), 125.49,125.40,125.10,122.94$ $\left(\mathrm{d}, J_{\mathrm{C}-\mathrm{P}}=3.7 \mathrm{~Hz}\right), 122.74,121.98,91.96\left(\mathrm{~d},{ }^{1} J_{\mathrm{C}-\mathrm{P}}=174.6 \mathrm{~Hz}\right), 52.81\left(\mathrm{~d},{ }^{2} J_{\mathrm{C}-\mathrm{P}}=3.7 \mathrm{~Hz}\right.$, $\left.\{\mathrm{PO}\} \mathrm{OCH}_{3} \times 2\right) ;{ }^{31} \mathrm{P} \mathrm{NMR}\left(121.5 \mathrm{MHz}, \mathrm{CDCl}_{3}\right) \delta 30.37$; HRMS for $\mathrm{C}_{20} \mathrm{H}_{17} \mathrm{O}_{4} \mathrm{P}$ : calcd. $\left(\mathrm{MH}^{+}\right)$: 353.0937, found: 353.0940

\section{Dimethyl (10-hydroxy-6,7-dimethoxyphenanthren-9-yl)phosphonate (5h)}

Brown solid; isolated yield 78\% (28 mg). $R_{f} 0.50$ (10\% EtOAc/hexane); Mp 135-137 ${ }^{\circ} \mathrm{C} ;{ }^{1} \mathrm{H}$ NMR $\left(300 \mathrm{MHz}, \mathrm{CDCl}_{3}\right) \delta 12.25(\mathrm{~d}, J=1.1 \mathrm{~Hz}, 1 \mathrm{H}), 8.46(\mathrm{~d}, J=8.2 \mathrm{~Hz}, 1 \mathrm{H}), 8.39(\mathrm{~d}, J=$ $8.3 \mathrm{~Hz}, 1 \mathrm{H}), 7.87(\mathrm{~d}, J=1.2 \mathrm{~Hz}, 1 \mathrm{H}), 7.66-7.72(\mathrm{~m}, 1 \mathrm{H}), 7.53(\mathrm{t}, J=7.8 \mathrm{~Hz}, 1 \mathrm{H}), 7.47(\mathrm{~s}$, $1 \mathrm{H}), 4.02(\mathrm{~s}, 3 \mathrm{H}), 3.94(\mathrm{~s}, 3 \mathrm{H}), 3.72\left(\mathrm{~d},{ }^{3} J_{\mathrm{H}-\mathrm{P}}=11.8 \mathrm{~Hz}, 6 \mathrm{H}\right) ;{ }^{13} \mathrm{C} \mathrm{NMR}\left(100 \mathrm{MHz}, \mathrm{CDCl}_{3}\right) \delta$ $162.12\left(\mathrm{~d},{ }^{2} J_{\mathrm{C}-\mathrm{P}}=6.7 \mathrm{~Hz}, \mathrm{C}-\mathrm{OH}\right), 149.89,147.26,133.37\left(\mathrm{~d}, J_{\mathrm{C}-\mathrm{P}}=2.1 \mathrm{~Hz}\right), 129.97,125.93$, $125.26\left(\mathrm{~d}, J_{\mathrm{C}-\mathrm{P}}=7.4 \mathrm{~Hz}\right), 124.88\left(\mathrm{~d}, J_{\mathrm{C}-\mathrm{P}}=1.2 \mathrm{~Hz}\right), 124.46\left(\mathrm{~d}, J_{\mathrm{C}-\mathrm{P}}=14.5 \mathrm{~Hz}\right), 121.96$, $120.11\left(\mathrm{~d}, J_{\mathrm{C}-\mathrm{P}}=11.6 \mathrm{~Hz}\right), 92.52\left(\mathrm{~d},{ }^{1} J_{\mathrm{C}-\mathrm{P}}=174.5 \mathrm{~Hz}\right), 56.00,55.82,52.78\left(\mathrm{~d},{ }^{2} J_{\mathrm{C}-\mathrm{P}}=4.1 \mathrm{~Hz}\right.$, $\left.\{\mathrm{PO}\} \mathrm{OCH}_{3} \times 2\right)$; ${ }^{31} \mathrm{P}$ NMR $\left(121.5 \mathrm{MHz}, \mathrm{CDCl}_{3}\right) \delta 30.18$; HRMS for $\mathrm{C}_{18} \mathrm{H}_{19} \mathrm{O} 6 \mathrm{P}$ : calcd. $\left(\mathrm{MH}^{+}\right)$: 363.0992, found: 363.0986

\section{Dimethyl (10-hydroxy-3-(trifluoromethyl)phenanthren-9-yl)phosphonate (5i)}

White solid; isolated yield $68 \%$ (25 mg). $R_{f} 0.50$ (10\% EtOAc/hexane); Mp 114-116 ${ }^{\circ} \mathrm{C} ;{ }^{1} \mathrm{H}$ NMR $\left(300 \mathrm{MHz}, \mathrm{CDCl}_{3}\right) \delta 12.60(\mathrm{~d}, J=0.4 \mathrm{~Hz}, 1 \mathrm{H}), 8.61(\mathrm{~d}, J=8.6 \mathrm{~Hz}, 1 \mathrm{H}), 8.55(\mathrm{~d}, J=$ $8.3 \mathrm{~Hz}, 1 \mathrm{H}), 8.51(\mathrm{dd}, J=8.2 \mathrm{~Hz}, 1.1 \mathrm{~Hz}, 1 \mathrm{H}), 8.27(\mathrm{~s}, 1 \mathrm{H}), 7.74-7.80(\mathrm{~m}, 1 \mathrm{H}), 7.60-7.69$ (m, $2 \mathrm{H}), 3.74\left(\mathrm{~d},{ }^{3} J_{\mathrm{H}-\mathrm{P}}=11.7 \mathrm{~Hz}, 6 \mathrm{H}\right) ;{ }^{13} \mathrm{C} \mathrm{NMR}\left(125 \mathrm{MHz}, \mathrm{CDCl}_{3}\right) \delta 164.00\left(\mathrm{~d},{ }^{2} J_{\mathrm{C}-\mathrm{P}}=6.5 \mathrm{~Hz}\right.$, $\mathrm{C}-\mathrm{OH}), 132.93,130.69,130.29\left(\mathrm{~d}, J_{\mathrm{C}-\mathrm{P}}=7.4 \mathrm{~Hz}\right), 129.43\left(\mathrm{q}, J_{\mathrm{C}-\mathrm{F}}=31.8 \mathrm{~Hz}\right), 128.20,128.10$, $126.15\left(\mathrm{~d}, J_{\mathrm{C}-\mathrm{P}}=14.0 \mathrm{~Hz}\right), 125.06,124.37\left(\mathrm{q},{ }^{1} J_{\mathrm{C}-\mathrm{F}}=270.4 \mathrm{~Hz}\right), 123.67,122.83,122.30(\mathrm{q}$ appearing as t, $\left.J_{\mathrm{C}-\mathrm{F}}=4.3 \mathrm{~Hz}\right), 121.31\left(\mathrm{q}\right.$ appearing as d, $\left.J_{\mathrm{C}-\mathrm{F}}=3.3 \mathrm{~Hz}\right), 93.44\left(\mathrm{~d},{ }^{1} J_{\mathrm{C}-\mathrm{P}}=\right.$ $177.8 \mathrm{~Hz}), 52.96\left(\mathrm{~d},{ }^{2} J_{\mathrm{C}-\mathrm{P}}=4.5 \mathrm{~Hz},\{\mathrm{PO}\} \mathrm{OCH}_{3} \times 2\right) ;{ }^{31} \mathrm{P} \mathrm{NMR}\left(121.5 \mathrm{MHz}, \mathrm{CDCl}_{3}\right) \delta 28.38$; HRMS for $\mathrm{C}_{17} \mathrm{H}_{14} \mathrm{~F}_{3} \mathrm{O}_{4} \mathrm{P}$ : calcd. $\left(\mathrm{MH}^{+}\right)$: 371.0655 , found: 371.0654

\section{Details of X-ray Analysis of 3zb'}

Single crystal X-ray data for compound 3zb' was collected on the Bruker APEX-II CCD area-detector at $100(2) \mathrm{K}$. A good quality single crystal of size 0.19 x 0.13 x $0.11 \mathrm{~mm}$, was selected under a polarizing microscope and was mounted on a glass fibre for data collection. Data collection was performed using $\omega$-scans of at 100(2) K by Bruker APEX2. ${ }^{3}$ Cell determination, and data reduction was performed using the Bruker SAINT software. Structure solution and refinement were performed by using SHELX-97. ${ }^{4}$ Refinement of coordinates and anisotropic thermal parameters of non-hydrogen atoms were carried out by the full-matrix least-squares method. The hydrogen atoms attached to carbon atoms were generated with idealized geometries and isotropically refined using a riding model. 
Crystallization: Crystals of compound 3zb' were grown from the solvent DCM:MeOH (1:3) by slow evaporation method (Figure 3; Table 1).
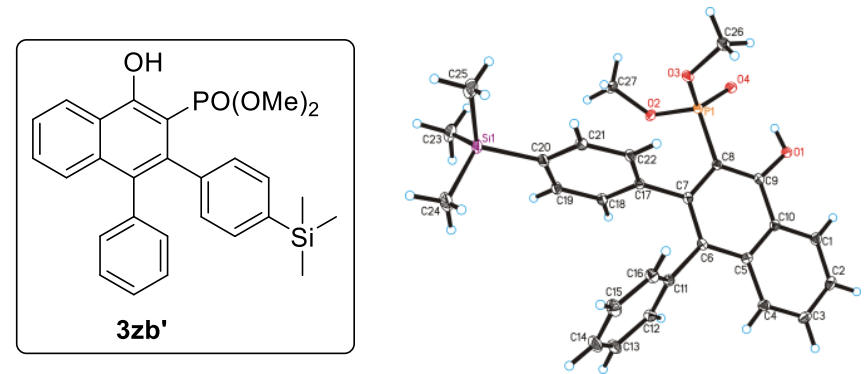

Figure 3 ORTEP diagram drawn with $30 \%$ ellipsoid probability for non-H atoms of the crystal structure of compound 3zb' determined at $273 \mathrm{~K}$.

Table 1. Crystal data and structure refinement details for $\mathbf{3 z b}$ '

\begin{tabular}{c|c}
\hline Compound & 3zb' \\
\hline Empirical formula & $\mathrm{C}_{27} \mathrm{H}_{29} \mathrm{O}_{4} \mathrm{P} \mathrm{Si}$ \\
Formula weight & 476.56 \\
Crystal System & Monoclinic \\
Space group & $P 21 / \mathrm{n}$ \\
$a(\AA)$ & $10.0339(5)$ \\
$b(\AA)$ & $25.2410(13)$ \\
$c(\AA)$ & $10.5446(6)$ \\
$\alpha\left(^{\circ}\right)$ & 90.00 \\
$\beta\left(^{\circ}\right)$ & $107.484(2)$ \\
$\gamma\left(^{\circ}\right)$ & 90.00 \\
$V\left(^{\circ}\right)$ & $2547.2(2)$ \\
$Z$ & 4 \\
$\mathrm{D}_{\mathrm{c}}\left(\mathrm{g} / \mathrm{cm}^{3}\right)$ & 1.243 \\
$F_{000}$ & 1008 \\
$\mu\left(\mathrm{mm}^{-1}\right)$ & 0.185 \\
$\theta$ max $\left({ }^{\circ}\right)$ & 28.27 \\
Total reflections & 39317 \\
Unique reflections & 6322 \\
Reflections $[I>2 \sigma(I)]$ & 4733 \\
Parameters & 304 \\
$R$ int & 0.0710 \\
Goodness-of-fit & 1.126 \\
$R\left[F^{2}>2 \sigma\left(F^{2}\right)\right]$ & 0.0481 \\
$w R\left(F^{2}\right.$, all data $)$ & 0.1522 \\
CCDC No. & 1916456 \\
\hline &
\end{tabular}

\section{References}

1. Pramanik, M. M. D.; Rastogi, N. Org. Biomol. Chem. 2016, 14, 1239.

2. Collomb, D.; Chantegrel, B.; Deshayes, C. Tetrahedron 1996, 52, 10455.

3. Krause, L.; Herbst-Irmer, R.; Sheldrick, G. M.; Stalke, D. J. Appl. Cryst. 2015, $48,3$.

4. Sheldrick, G. M. Acta Crystallogr. Sect. A 2008, 64, 112. 


\section{7. ${ }^{1} \mathrm{H},{ }^{13} \mathrm{C} \&{ }^{31} \mathrm{P}$ NMR spectra of products}

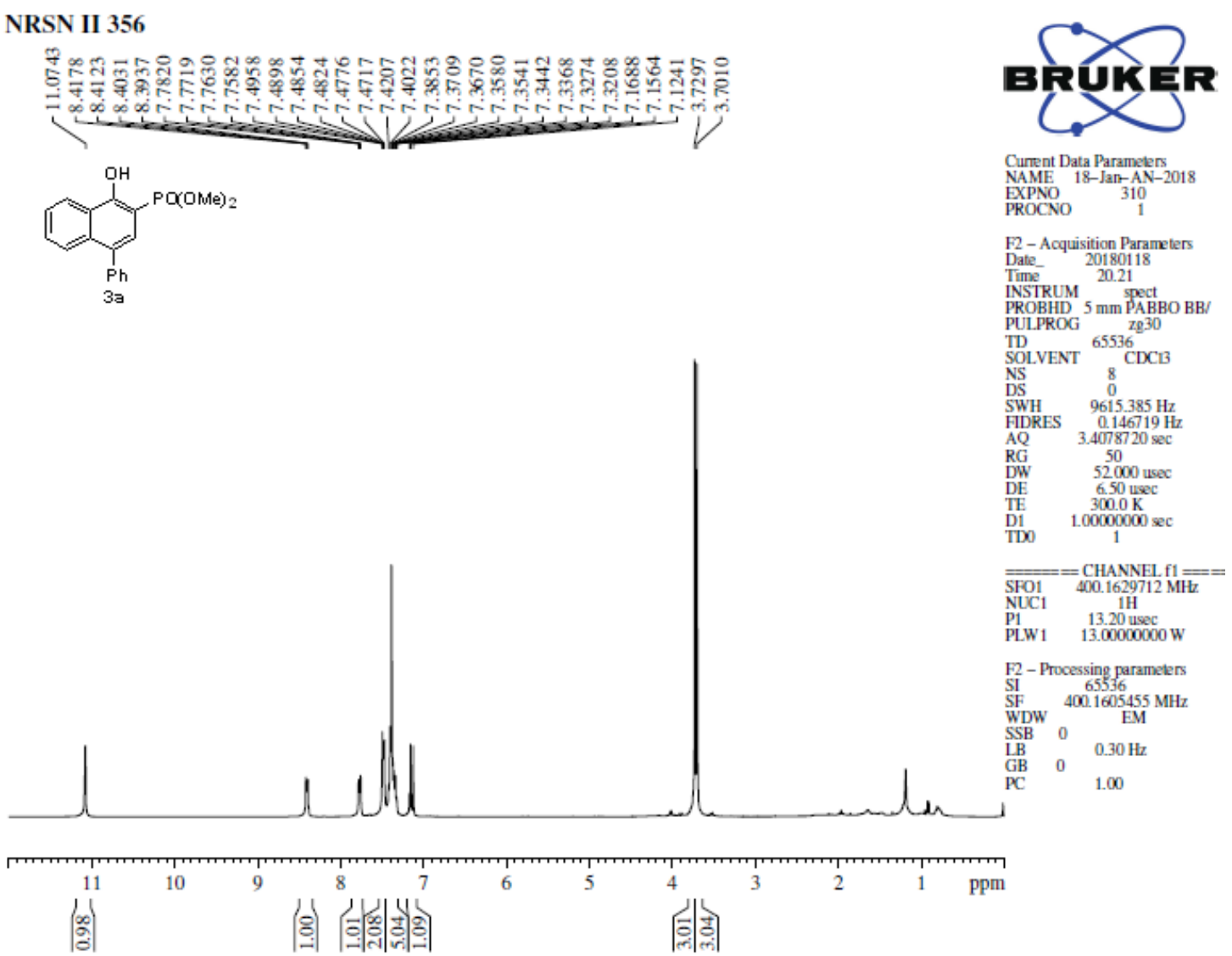

Spectrum 1: ${ }^{1} \mathrm{H}$ NMR spectrum of $\mathbf{3 a}$

NRSN-II-356
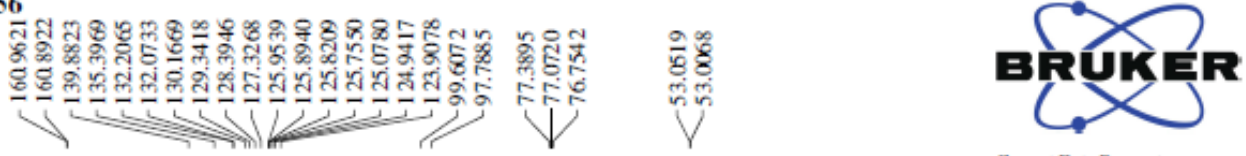

Current Data Paramekers

NAME $02-\mathrm{Feb}-\mathrm{AN}-2$ PRPNO 440

F2- Acquisition Parameters
Date_ 20180202

Date- 2018020

Time 16.18

PROBHD $5 \mathrm{~mm}$ PABBO BR

PULPROG ${ }^{2} \mathrm{Fpg}^{30}$

TD

NS 1024

NS

DS

FWHRES $\quad 24038.461 \mathrm{~Hz}$

AQ $\quad 1.3631488 \mathrm{sc}$

201.48

$3001 \mathrm{~K}$

$200000000 \mathrm{sec}$
$0.030000000 \mathrm{sec}$

Dil 0.03000000

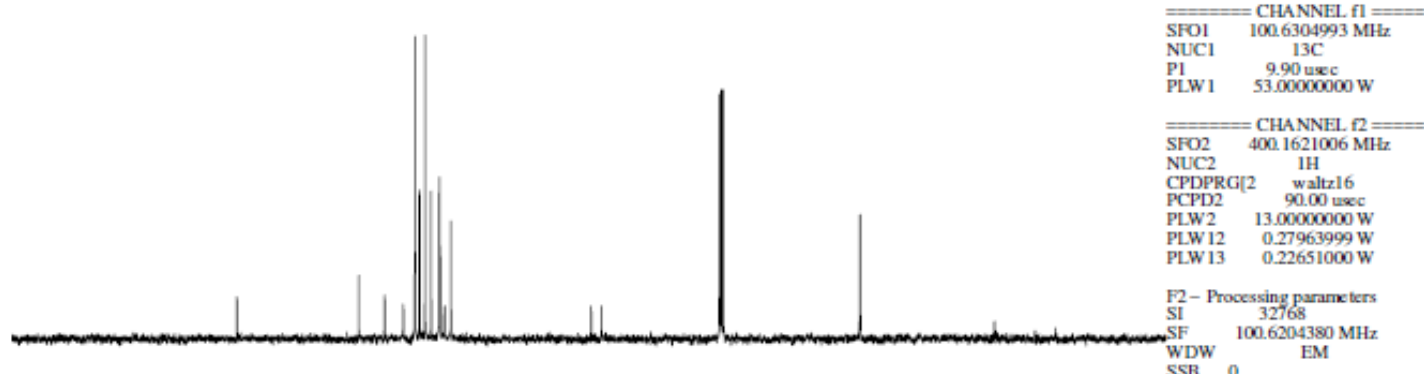

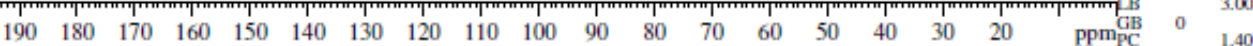

Spectrum 2: ${ }^{13} \mathrm{C}$ NMR spectrum of $\mathbf{3 a}$ 
NRSN-II-356

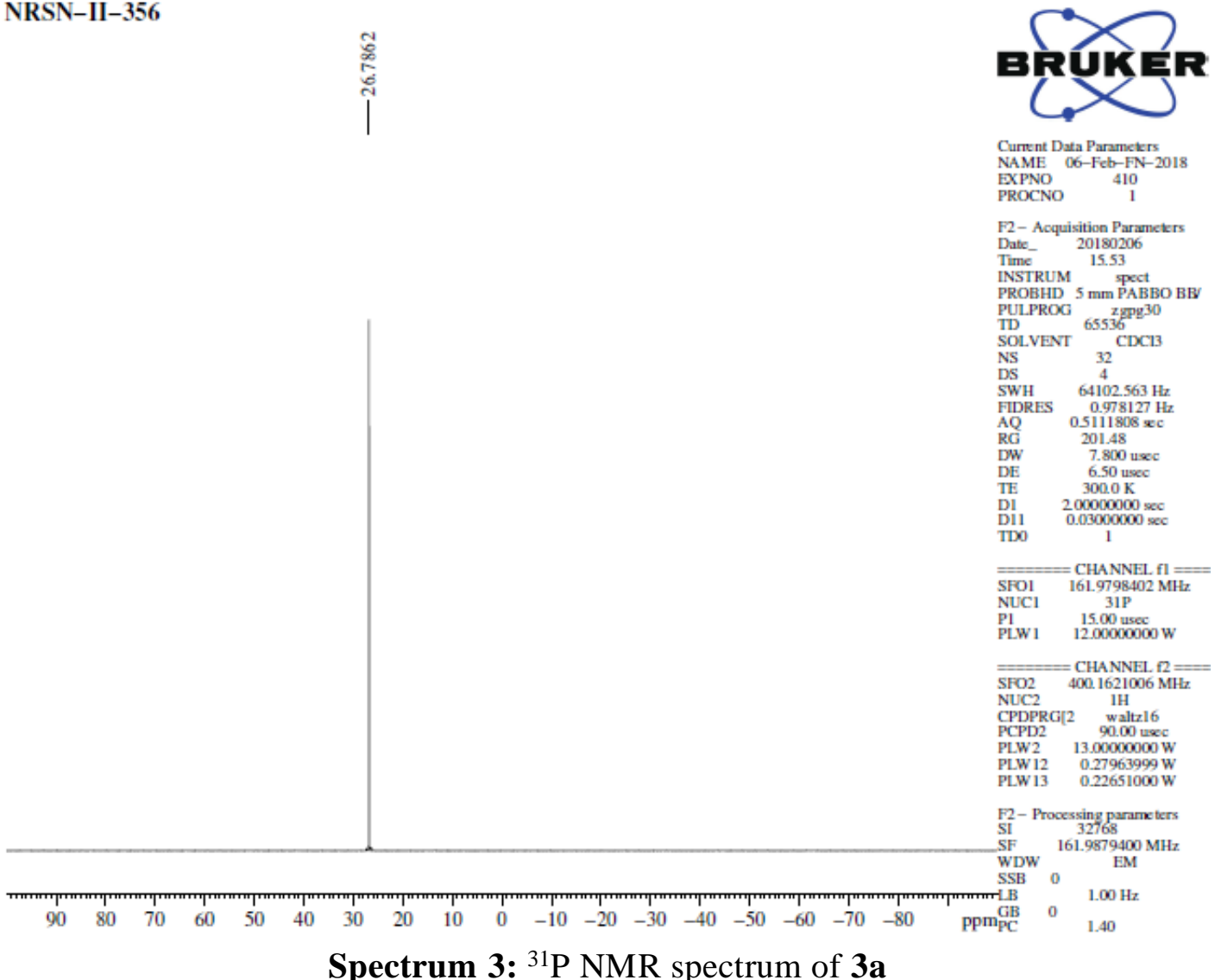

NRSN II 377

Spectrum 3: ${ }^{31} \mathrm{P}$ NMR spectrum of $\mathbf{3 a}$
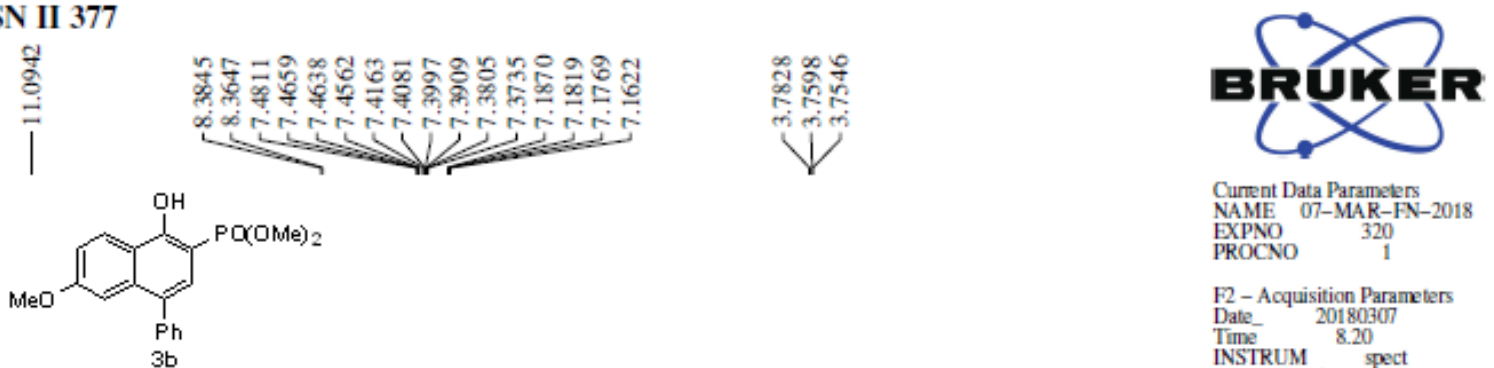

Cunent Data Parameters
NAME $07-$ MAR-FN-2018

EXPNO 320

F2 - Acquisition Parameters

$\begin{array}{ll} & 2018030 \\ \text { Time } & 8.20\end{array}$

INSTRUM spect

PROBHD 5 mm PATXI $1 \mathrm{H}$

$\begin{array}{ll}\text { PULPROG } & \text { Zg } 30 \\ 65536\end{array}$

SDLLVENT $_{8}^{65536} \mathrm{CDCB}$

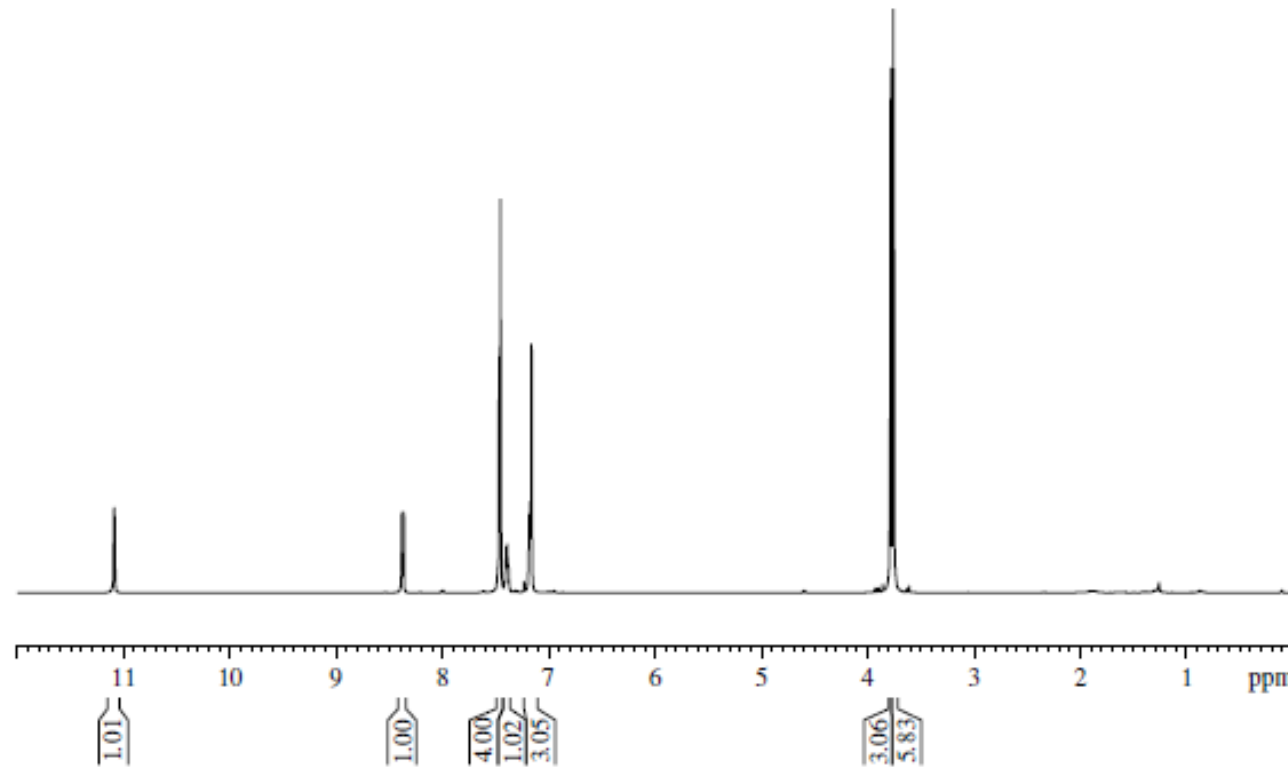

$\begin{array}{ll}\text { NS } & 8 \\ \text { DS } & 2\end{array}$

SWH $\quad 12019.230 \mathrm{~Hz}$

FWRES $0.183399 \mathrm{~Hz}$

AQ $\quad 2.7262976 \mathrm{sec}$

$\begin{array}{ll}\text { RG } & 19.72 \\ \text { DW } & 41600\end{array}$

DE $\quad 6.50$ usec

TE $\quad 303.0 \mathrm{~K}$

$\begin{array}{lc}\text { D1 } & 1.00000000 \mathrm{sec} \\ \text { TD0 } & 1\end{array}$

$\overline{\text { FO1 }}=\underset{500.6794419 \mathrm{MHz}}{\text { CHANN }} \mathrm{f1}=$

NUC1 500.6794410

$\begin{array}{lc}\text { P1 } & 6.45 \text { usec } \\ \text { PLW1 } & 13.60000038 \mathrm{~W}\end{array}$

F2 - Processing parameters

$\begin{array}{lc}\text { SI } & 65536 \\ \text { SF } & 500.6763748 \mathrm{MHz}\end{array}$

WDW EM

$\underset{\mathrm{LB}}{\mathrm{LSB}} 0.30 \mathrm{~Hz}$

PC $\quad 1.00$

Spectrum 4: ${ }^{1} \mathrm{H}$ NMR spectrum of $\mathbf{3 b}$ 


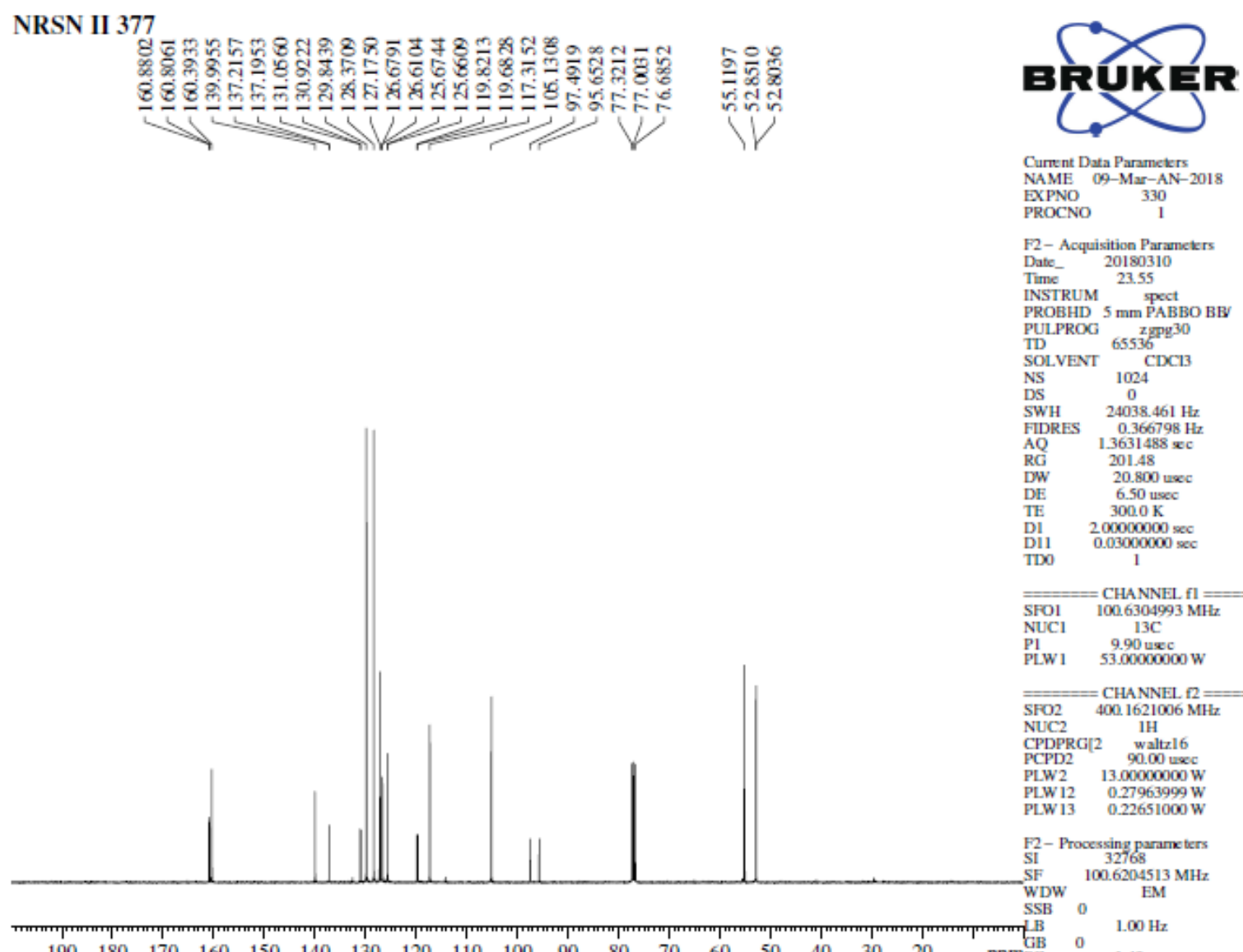

$\begin{array}{lllllllllllllllllllll}190 & 180 & 170 & 160 & 150 & 140 & 130 & 120 & 110 & 100 & 90 & 80 & 70 & 60 & 50 & 40 & 30 & 20 & \mathrm{PpmPC} & 0 & 1.40\end{array}$

Spectrum 5: ${ }^{13} \mathrm{C}$ NMR spectrum of $\mathbf{3 b}$

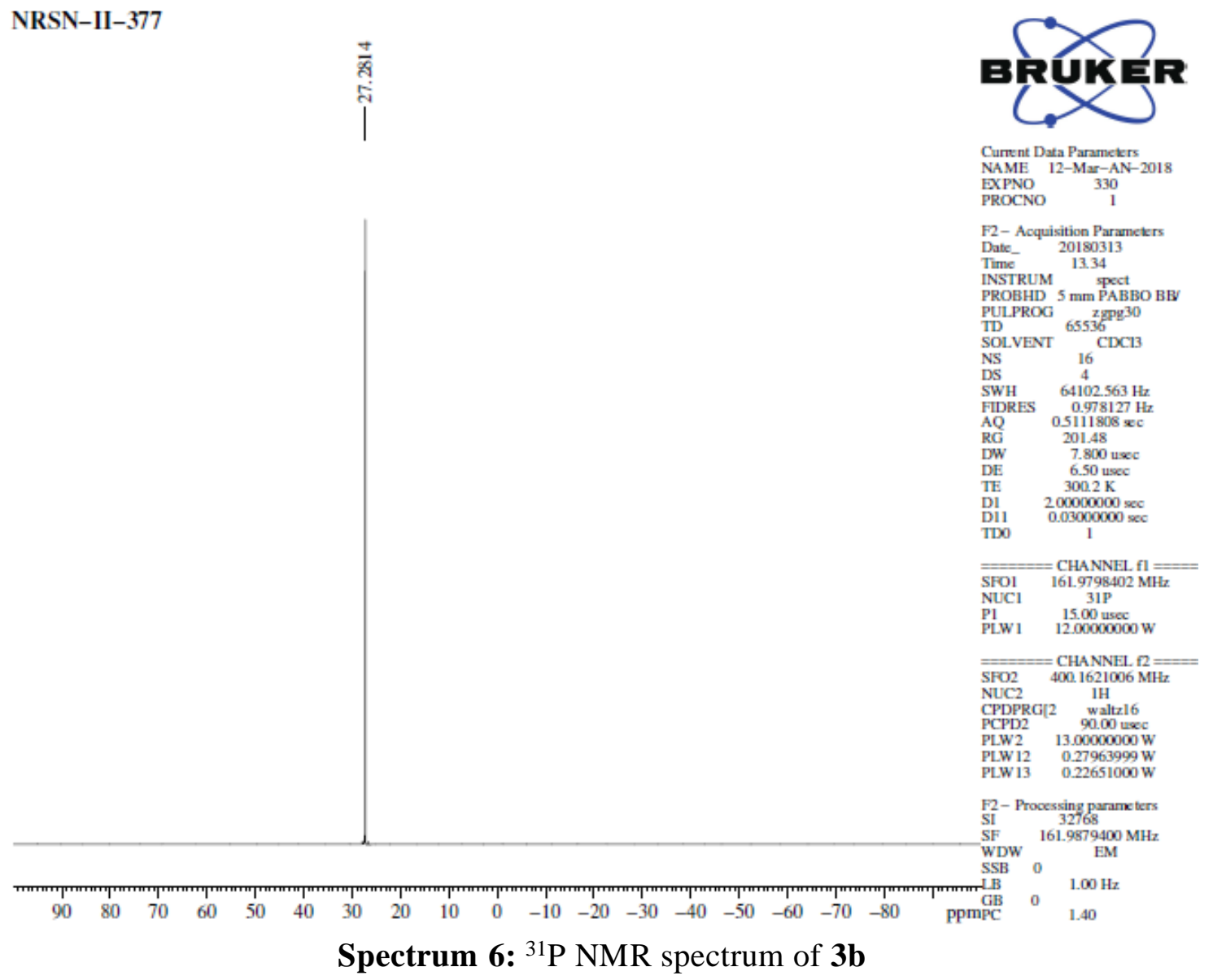




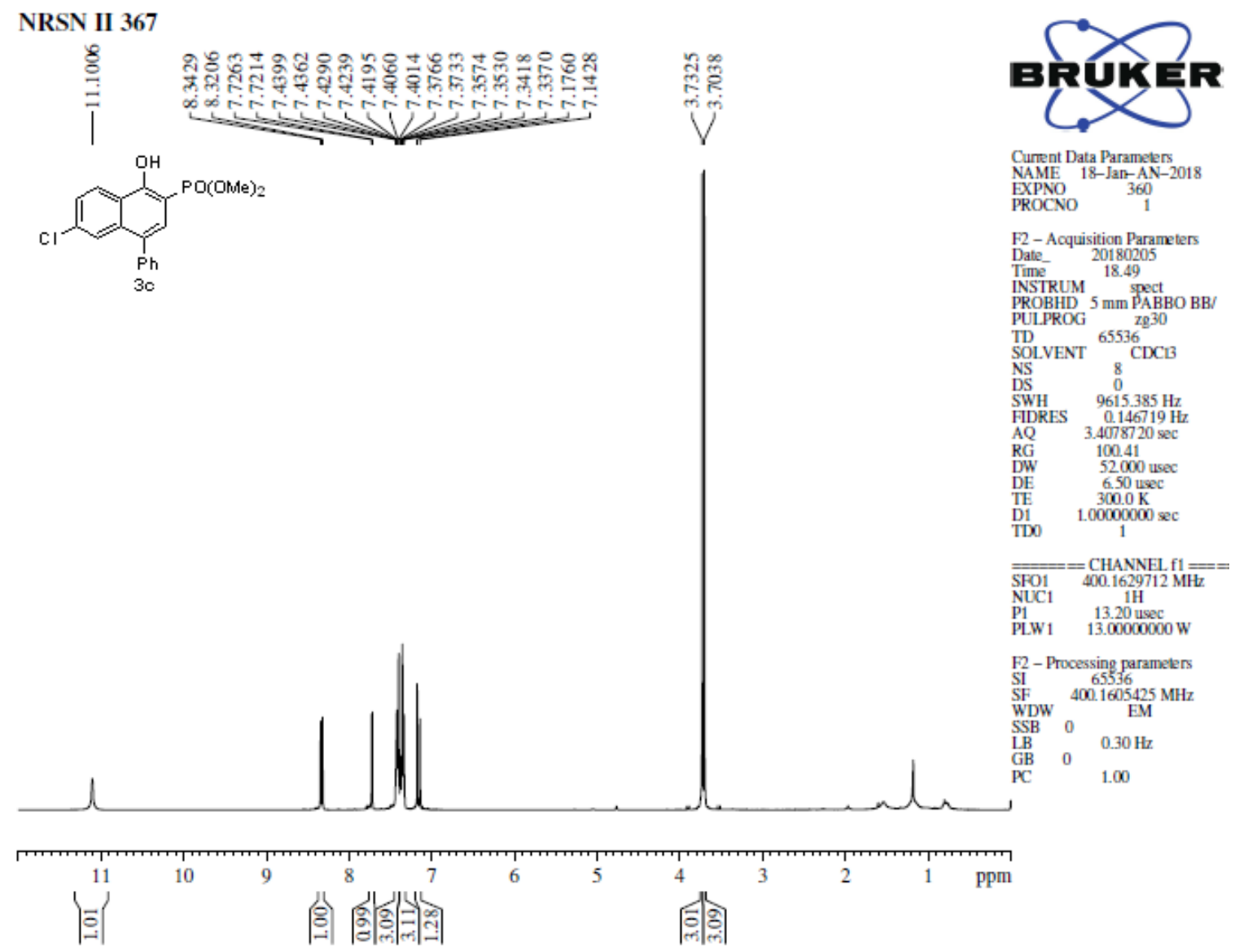

Spectrum 7: ${ }^{1} \mathrm{H}$ NMR spectrum of $3 \mathrm{c}$

NRSN II 367
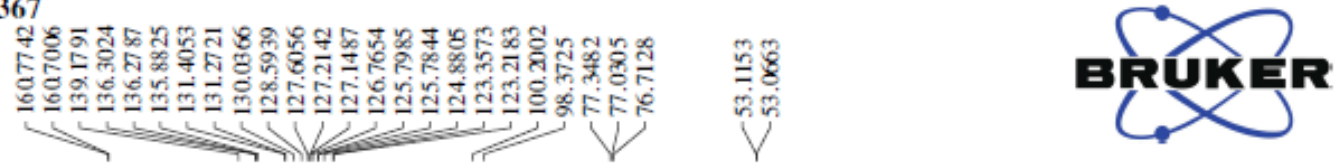

Current Data Paramekers

EXPNO

PROCNO 1

F2- Acquisition Parameters

Dabe_ 2018020

INSTRUM 19.05

$\begin{array}{ll}\text { INSTRUM } & \text { spect } \\ \text { PROBHD } 5 \text { mm PABBO BB } & \end{array}$

PULPROG $2 \mathrm{z}^{2} \mathrm{Fg}^{30}$

TD 65536 -

NS

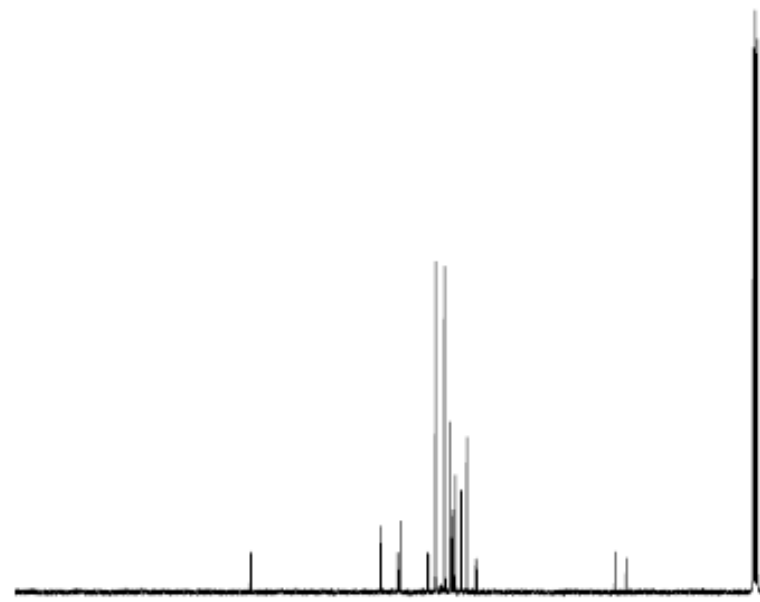

NS 1024

$\begin{array}{lc}\text { DS } & 0 \\ \text { SWH } & 24038.461 \mathrm{~Hz}\end{array}$

$\begin{array}{ll}\text { FWDRES } & 0.366798 \mathrm{H} \\ \mathrm{AOO} & 13631488 \mathrm{xc}\end{array}$

$\begin{array}{ll}\text { AQ } & 1.3631488 \\ \text { RG } & 201.48\end{array}$

DW $\quad 20.800$ uxe:

TE $\quad 3.50$ u

$\begin{array}{ll}\text { D1 } & 2000000000 \\ \text { Dil } & 0.03000000\end{array}$

CHANNEL $\mathrm{fl}=$

SFOl $100.6304993 \mathrm{M}$

$\begin{array}{ll}\text { Pl } & 9.9000 \mathrm{c} \\ \text { PLW1 } & 53.00000000 \mathrm{~W}\end{array}$

CHANNEL $\mathrm{R}=$

$\mathrm{SFO} 2 \quad 400.1621006 \mathrm{MH}$

CPDPRG12 1H

PCPD2 20 waltz16

$1300000000 \mathrm{~W}$

$\begin{array}{lll}\text { PLW } 12 & 0.27963999 \mathrm{~W}\end{array}$

PLW13 $0.22651000 \mathrm{~W}$

S2- Processing parane ters $\begin{array}{ll}32768 \\ \mathrm{SF} & 100.6204380 \mathrm{MHz}\end{array}$ SSB

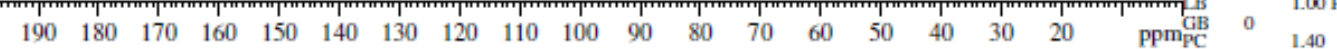

Spectrum 8: ${ }^{13} \mathrm{C}$ NMR spectrum of $\mathbf{3 c}$ 


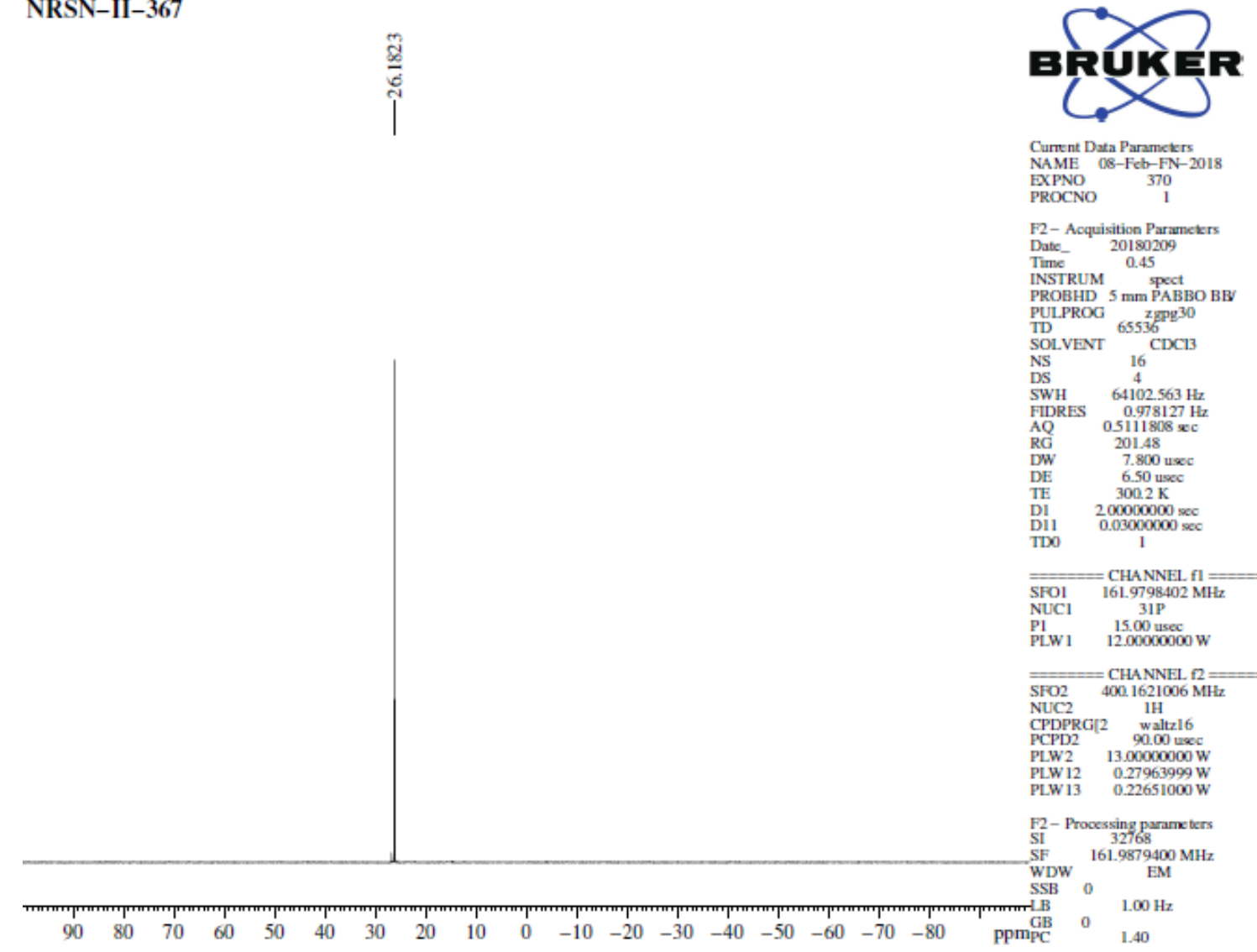

Spectrum 9: ${ }^{31} \mathrm{P}$ NMR spectrum of $\mathbf{3 c}$

NRSN II 369

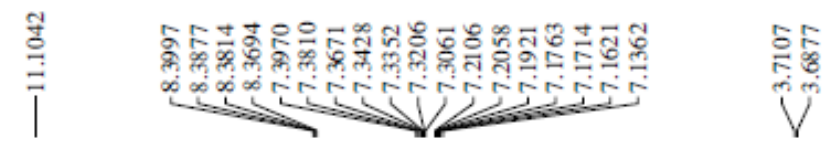<smiles>COc1cc(-c2ccccc2)c2cc(F)ccc2c1O</smiles>

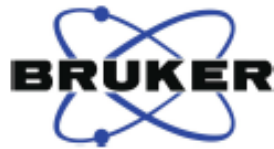

Cunent Data Paramelers NAME 07-MAREXPNO

F2-Acquisition Parameters

$\begin{array}{lc}\text { Date_- } & 2018030 \\ \text { Time } & 8.15\end{array}$

Time
INSTRUM
PROBHD
PR 5 mect
PATXI $1 \mathrm{H}$

PULPROG zg. 30

$\begin{array}{ll}\text { TD } & \\ \text { SOLVENT } & 65536 \\ \mathrm{CDCl}\end{array}$

NS

$\begin{array}{ll}\text { DS } & \stackrel{2}{2} .230 \mathrm{~Hz}\end{array}$

$\begin{array}{lr}\text { SWH } & 12019.230 \mathrm{~Hz} \\ \text { FIDRES } & 0.183399 \mathrm{~Hz}\end{array}$

AQ $27262976 \mathrm{sec}$

$\begin{array}{ll}\mathrm{RG} & 30.83 \\ \mathrm{DW} & 41.600\end{array}$

DE $\quad 6.50$ usec

TE $\quad 303.1 \mathrm{~K}$

D1 100000000

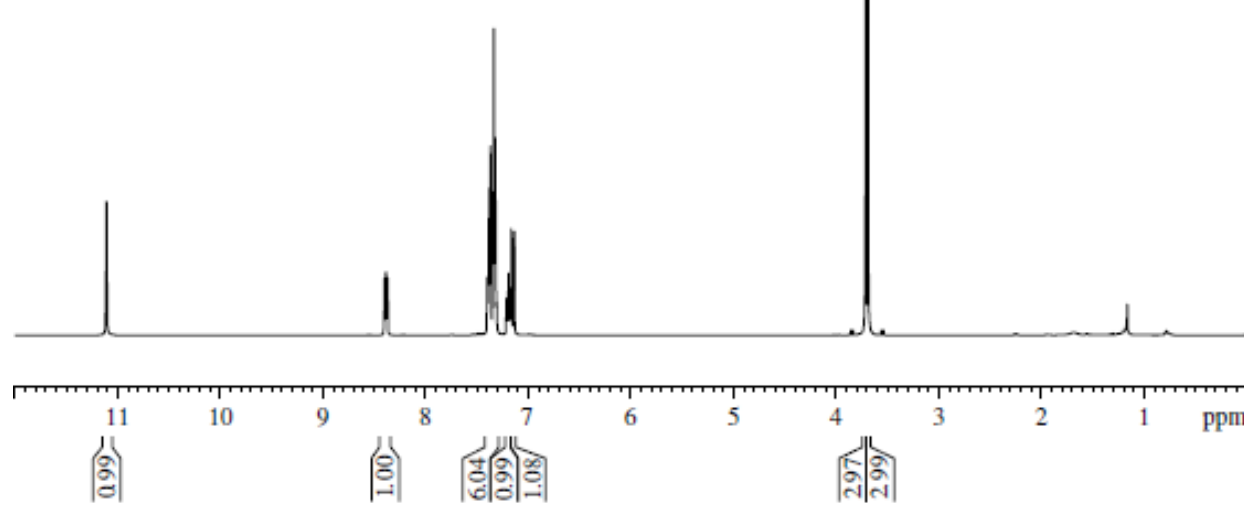

$\overline{\overline{S F O 1}}=\underset{500.6794419 \mathrm{MHz}}{\text { CHANNEI }} \mathrm{f1}=$

NUC1 iH

$\begin{array}{ll}\text { P1 } & 6.45 \text { usec } \\ \text { PLW1 } & 13.60000038 \mathrm{~W}\end{array}$

F2 - Processing parameters

$\begin{array}{lc}\text { SI } & 65536 \\ \text { SF } & 500.6764175 \mathrm{MHz}\end{array}$

SSB 0

LB $0.30 \mathrm{~Hz}$

$\begin{array}{lll}\mathrm{GB} & 0 & \\ \mathrm{PC} & & 1.00\end{array}$

Spectrum 10: ${ }^{1} \mathrm{H}$ NMR spectrum of $\mathbf{3 d}$ 

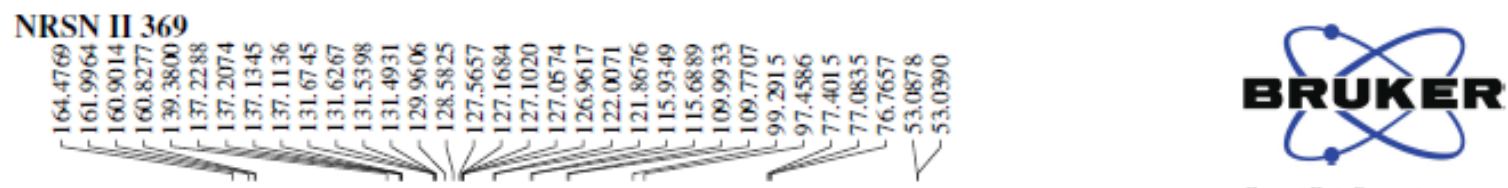

Curnent Data Parameters

$\begin{array}{lc}\text { NAME } & 09-\mathrm{Mar}-\mathrm{AN}-2018 \\ \text { EXPNO } & 340 \\ \text { PROCNO } & 1\end{array}$

F2- Acquisition Parameters

Dabe_ 2018031

Time

PROBHD 5 mm PABBO BE

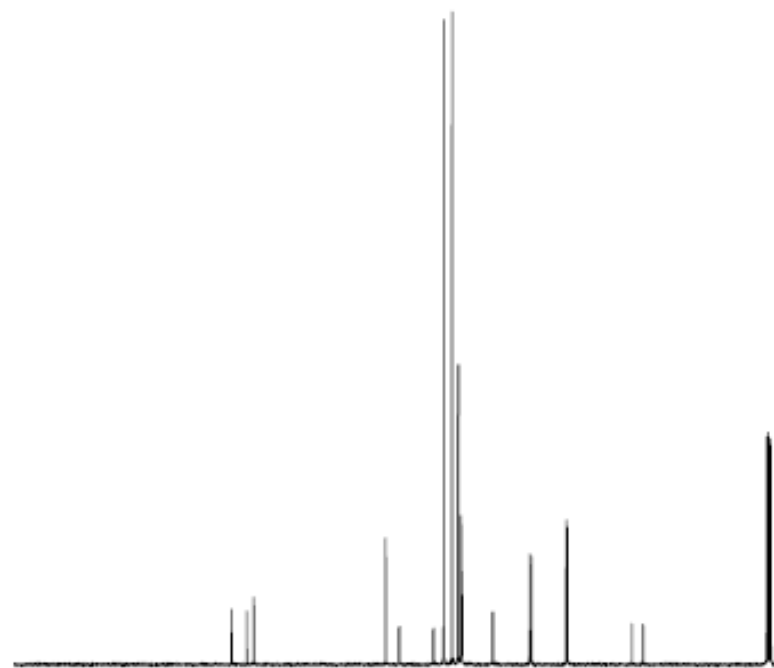

PULPROG

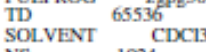

$\begin{array}{lc}\mathrm{NS} & 102 \\ \mathrm{DS} & 0\end{array}$

$\begin{array}{lc}\text { DS } & 0 \\ \text { SWH } & 24038.461 \mathrm{~Hz}\end{array}$

FQ 1.3631488 xec

RG 201.48

DE $\quad 6.50$ usec

TE $3000 \mathrm{~K}$

DI 200000000 sec

$\begin{array}{lr}\text { DI1 } & 0.0300000 \\ \text { TD0 } & 1\end{array}$

CHANNEL $\mathrm{fl}=$

SFOI $100.6304993 \mathrm{ML}$

Pl 9.90 use

PhW $53.00000000 \mathrm{~W}$

CHANNEL $R=$

NUC2 $202.1 \mathrm{H}$

CPDPRG[2 waltzl6

PCPD2 90.00 usec
PLW/ $13.00000000 \mathrm{~W}$

$\begin{array}{lr}\text { PLW2 } & 13.00000000 \mathrm{~W} \\ \text { PLW12 } & 027963999 \mathrm{~W}\end{array}$

PLW 13 $0.22651000 \mathrm{~W}$

F2- Processing parame ters

SF $\quad 100.6204380 \mathrm{MHz}$

WDW EM

SSB $0 \quad 1.00 \mathrm{H}$

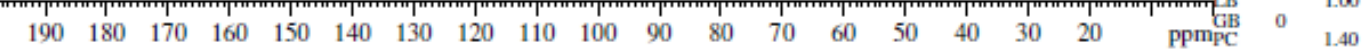

Spectrum 11: ${ }^{13} \mathrm{C}$ NMR spectrum of $\mathbf{3 d}$

NRSN-II-369

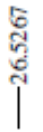

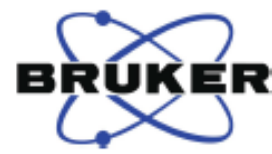

Current Data Paramekers

$\begin{array}{lc}\text { EXPNO } & 340 \\ \text { PROCNO } & 1\end{array}$

F2- Acquisition Parameters

Date_ 20180313

Time

PROBHD $5 \mathrm{~mm}$ PABBO B

PULPROG $25 \mathrm{zpg}^{30}$

TD $65536 \mathrm{C}$

SOLVENT

DS

SWH $\quad 64102.563 \mathrm{~Hz}$

FDRES $0.978127 \mathrm{H}$

AQ $\quad 0.5111808 \times$

$\begin{array}{ll}\text { DW } & 7.800 \text { usec } \\ \text { DE } & 6.50 \text { usee }\end{array}$

6.50 usec
$300.1 \mathrm{~K}$

$200000000 \mathrm{sec}$

$\begin{array}{ll}\text { DI } & 200000000 \mathrm{sec} \\ \text { Dil } & 0.03000000 \mathrm{sec}\end{array}$

SFOI $161.9798402 \mathrm{MHz}$

NUCl 31P

P1 15.00 usec
PLW1 1200000000 .

CHANNEL $\mathrm{f} 2=$

$\begin{array}{ll}\mathrm{SFO} 2 & 400.1621006 \mathrm{MH}\end{array}$

NUC2 2

CPDPRG[2
PCPD2

PLW 2 13,00000000

PLW13 $0.22651000 \mathrm{~W}$

2 - Processing parame ters SF $\quad 161.9879400 \mathrm{MHz}$ 


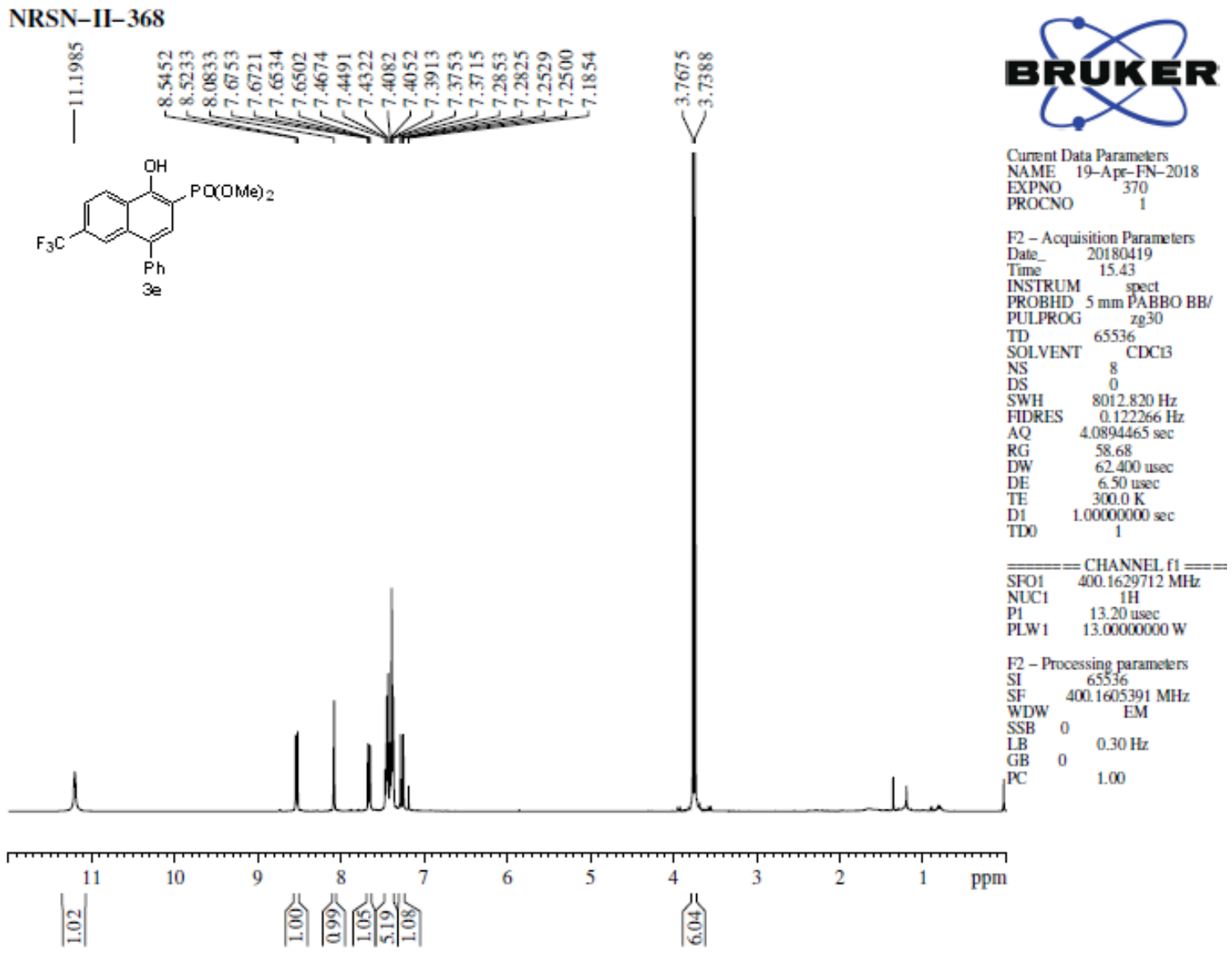

Spectrum 13: ${ }^{1} \mathrm{H}$ NMR spectrum of $\mathbf{3 e}$

NRSN II 368

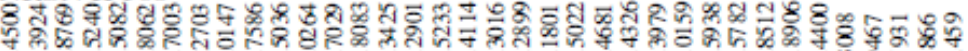

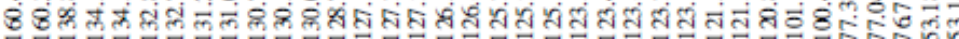

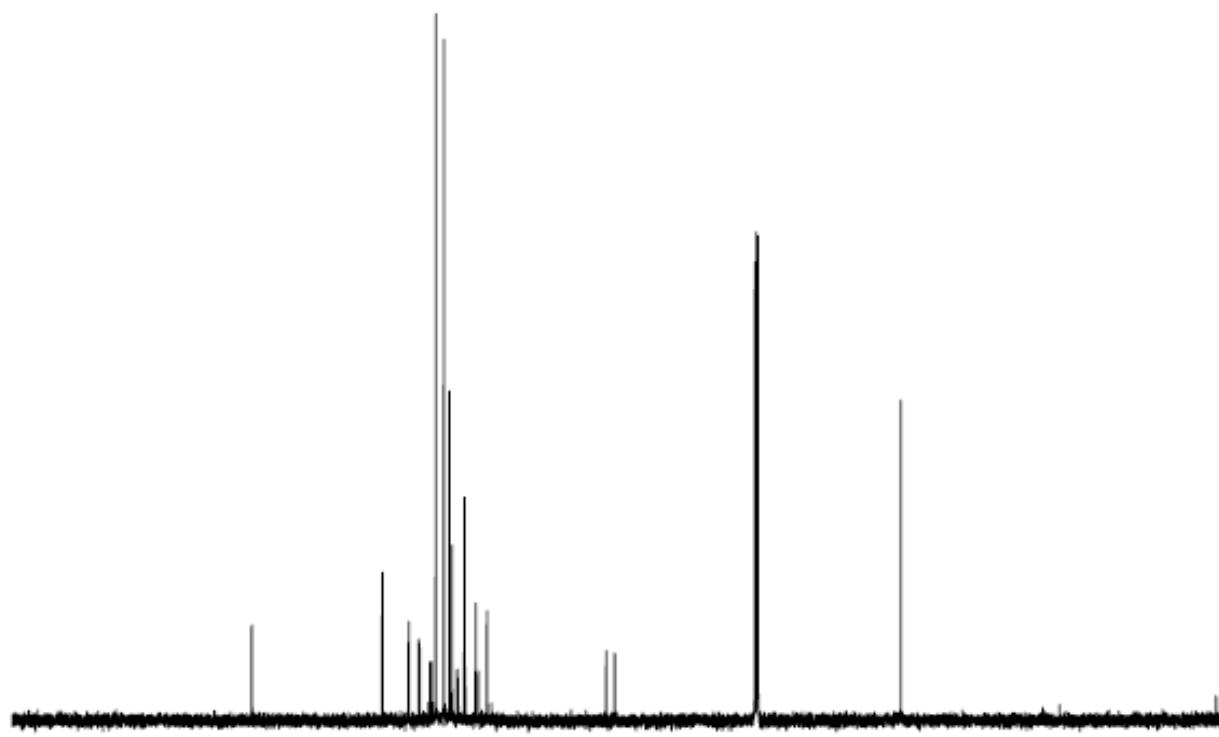

PRUKER

Cunent Data Parameters
NAME 23 -APR-AN-2018

EXPNO

F2 - Acquisition Parameters

F2 - Acquisition Para
Date_ $\quad 20180423$

Time 1200

INSTRUM spect

PUL.PROG ZRPg30

ID 65536

NS

$\begin{array}{lc}\text { NS } & 2048 \\ \text { DS } & 4\end{array}$

SWH $\quad 29761.904 \mathrm{~Hz}$

FIDRES $\quad 0.454131 \mathrm{H}$

AQ $1.1010048 \mathrm{sec}$

$\begin{array}{ll}\text { RG } & 81.53 \\ \text { DW } & 16.800 \text { use }\end{array}$

. 6.50 usec

$0 \mathrm{~K}, 0000000 \mathrm{xec}$

Di1 $0.03000000 \mathrm{sec}$

TIDO 1

$\overline{S F O 1}=$ CHANNE $\mathrm{fl}=$

SFO1 125.9077573 MH

$973 \mathrm{C}$

$\begin{array}{ll}\text { P1 } & 9.23 \text { usec } \\ \text { PLW1 } & 244.00000000 \mathrm{~W}\end{array}$

$\overline{\mathrm{SFO} 2}=\underset{500.6783527 \mathrm{MHz}}{\text { CHANNE }} \mathrm{f}=$

NUC2 $1 \mathrm{H}$

CPDPRG 2 waltz16

DLW2 $13.60000038 \mathrm{~W}$

LW $13 \quad 0.05657900 \mathrm{~W}$

F2 - Processing parameters $125.8951680 \mathrm{MHz}$

$\begin{array}{llllllllllllllllllll}190 & 180 & 170 & 160 & 150 & 140 & 130 & 120 & 110 & 100 & 90 & 80 & 70 & 60 & 50 & 40 & 30 & 20 & \mathrm{ppm} & 0 \\ \mathrm{GC} & 0 & 1.40\end{array}$

Spectrum 14: ${ }^{13} \mathrm{C}$ NMR spectrum of $3 \mathbf{e}$ 


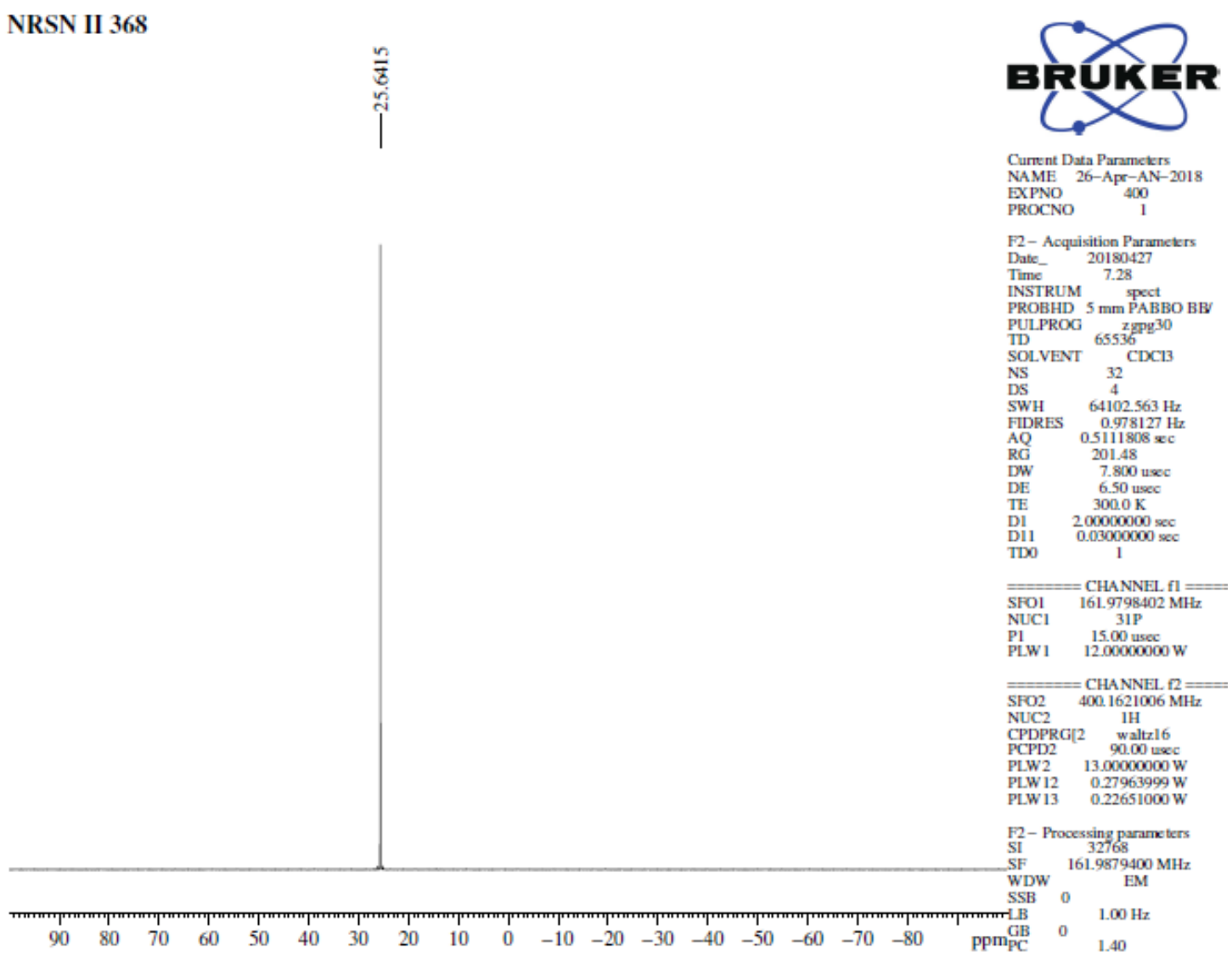

Spectrum 15: ${ }^{31} \mathrm{P}$ NMR spectrum of $3 \mathbf{e}$

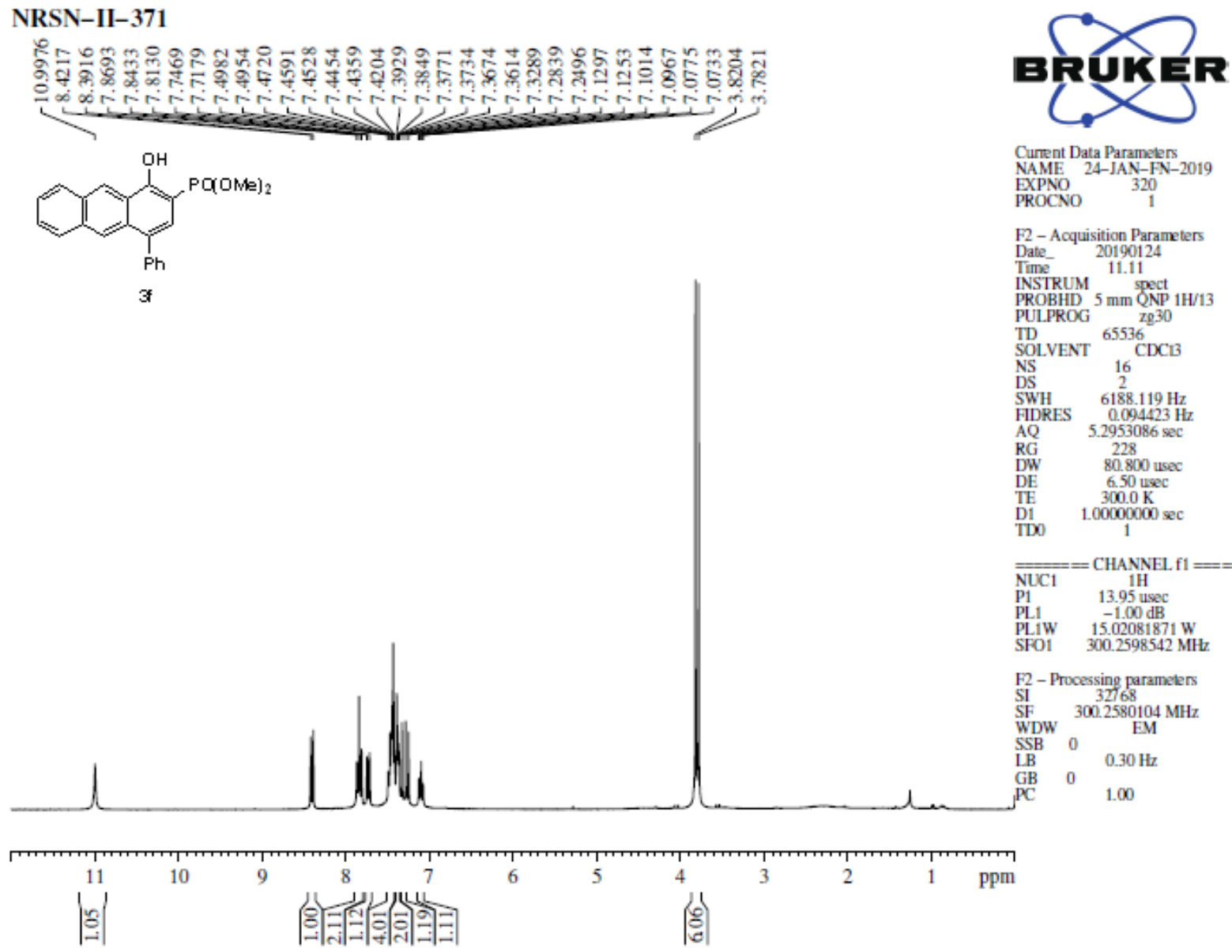

Spectrum 16: ${ }^{1} \mathrm{H}$ NMR spectrum of $\mathbf{3 f}$ 


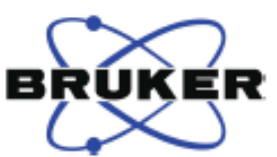

Curnent Data Parameters
NAME
15-Mar-AN-2018

EXPNO

F2- Acquisition Paramekers

Date_ 20180316

Time- 15.08

$\begin{array}{lll}\text { INSTRUM } & \text { spect } \\ \text { PROBHD } & 5 \mathrm{~mm} \text { PABBO BE }\end{array}$

PROBHD $5 \mathrm{~mm}$ PABBO

TD $65536 \%$

SOLVENT CDCII

NS $\quad 1024$

$\begin{array}{ll}\text { DS } & 4 \\ \text { SWH } & 24038.461 \mathrm{~Hz}\end{array}$

FIDRES $\quad 0.366798 \mathrm{~s}$

AQ 1.3631488 se

RG 201.48

$\begin{array}{ll}\text { DW } & 20.800 \text { used } \\ \text { DE } & 6.50 \text { usec }\end{array}$

$300.0 \mathrm{~K}$

Dil $\quad 0.03000000 \mathrm{sec}$

TDO 1

CHANNEL $\mathrm{fl}=$

SFO1 $100.6304993 \mathrm{MH}$

NuCl $13 \mathrm{C}$

$\begin{array}{ll}\text { Pl } & 9.90 \text { uxec } \\ \text { PLW1 } 53.00000000 \mathrm{~W}\end{array}$

CHANNEL $\mathrm{R2}=$

SFO2 $400.1621006 \mathrm{MHz}$

NUC2 $21 \mathrm{H}$

CPDPRG 2 waltz16

PLW2 $13,00000000 \mathrm{~W}$

PLW 12 $0.27963999 \mathrm{~W}$

PLW $13 \quad 0.22651000 \mathrm{~W}$

F2- Processing parame ters $\begin{array}{ll}\mathrm{SI} & 32768 \\ \mathrm{SF} & 100.6204380 \mathrm{MHz}\end{array}$

WDW

B $100 \mathrm{H}$

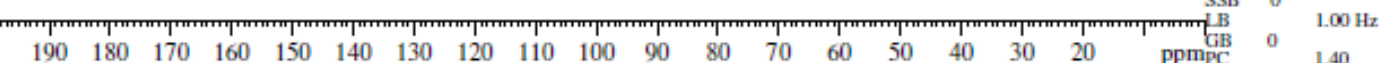

$\begin{array}{llllllllllllllllllllll}190 & 180 & 170 & 160 & 150 & 140 & 130 & 120 & 110 & 100 & 90 & 80 & 70 & 60 & 50 & 40 & 30 & 20 & \text { PpmP } & 0 & 1.40\end{array}$

Spectrum 17: ${ }^{13} \mathrm{C}$ NMR spectrum of $\mathbf{3 f}$

NRSN-II-371
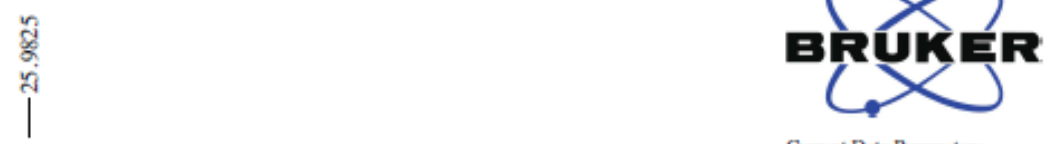

Current Data Paramekers
NAME 19-Mar-AN-2018

EXPNO

PROCNO

1

F2- Acquisition Paramekers

Time 8.32

PROBHD $5 \mathrm{~mm}$ РABBO BE

PULPROG 2 pgig 30

TD $65536{ }^{2}$

NS $16^{\text {CDC13 }}$

$\begin{array}{ll}\text { NS } & 16 \\ \text { DS } & 4 \\ \text { SWH } & 64102563 \mathrm{H}\end{array}$

SW7 $64102.563 \mathrm{~Hz}$

AQ $0.5111808 \times$

RG 201.48

$\begin{array}{ll}\mathrm{DW} & 7.800 \mathrm{usec} \\ \mathrm{DE} & 6.50 \mathrm{usec}\end{array}$

$300.2 \mathrm{~K}$

$200000000 \mathrm{sec}$

Di1 $0.03000000 \mathrm{sec}$

TDO

CHANNEL $\mathrm{fl}=$

SFOI 161.9798402

NiP $31 \mathrm{P}$

$\begin{array}{ll}\text { Pl } & 15.00 \text { usec } \\ \text { PLW1 } & 12.00000000 \mathrm{~W}\end{array}$

$=$ CHANNEL $R 2=$

$\begin{array}{ll}\mathrm{SFO} 2 & 400.1621006 \mathrm{MH}\end{array}$

$\mathrm{NUC2} 21 \mathrm{H}$

CPDPRG12 waltz16

PLW2 $1300000000 \mathrm{~W}$

PLW $13 \quad 0.22651000 \mathrm{~W}$

F2- Processing paranketers $161.9879400 \mathrm{MH}$

WDW

SSB 0

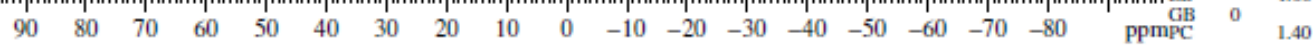

Spectrum 18: ${ }^{31} \mathrm{P}$ NMR spectrum of $\mathbf{3 f}$ 


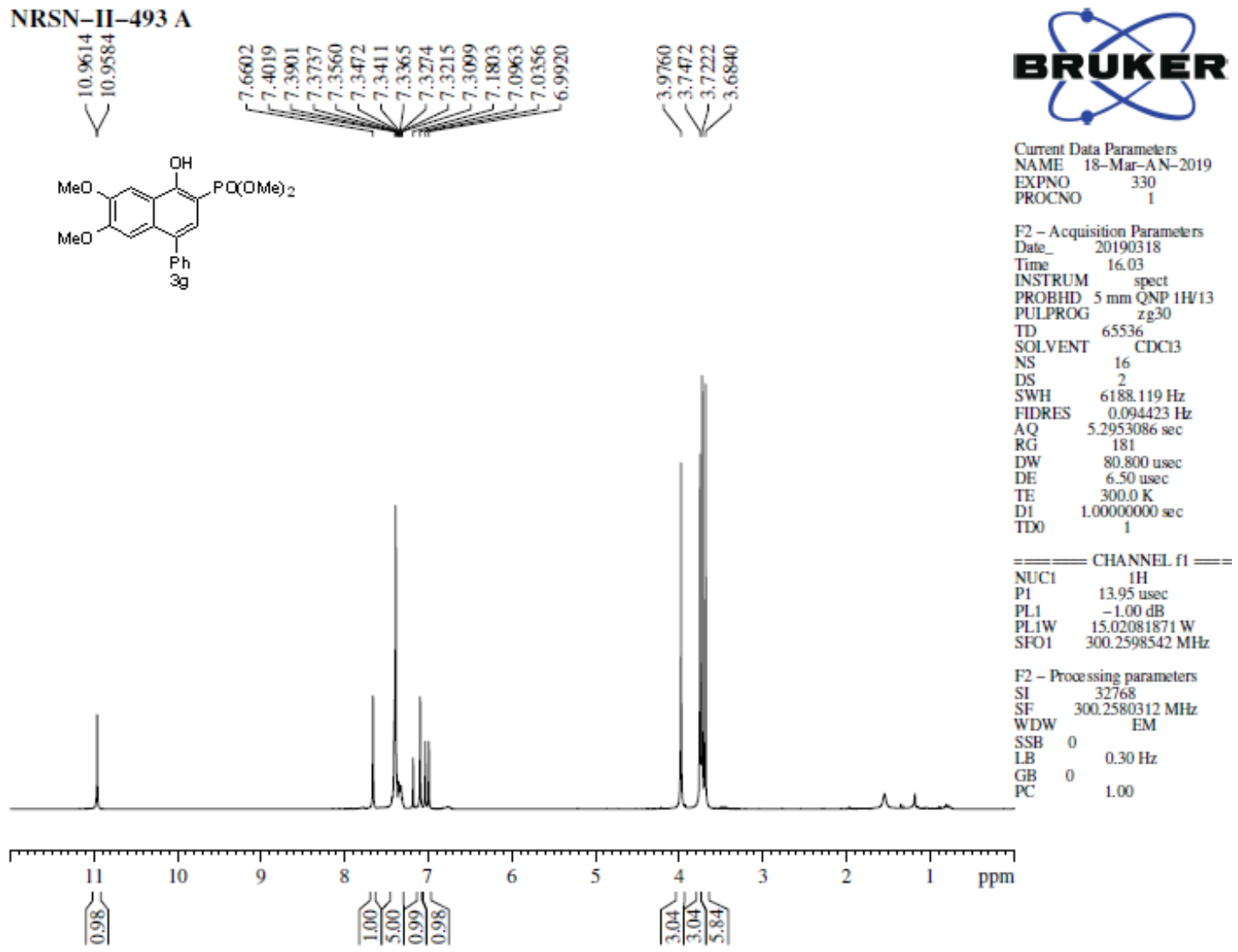

Spectrum 19: ${ }^{1} \mathrm{H}$ NMR spectrum of $\mathbf{3 g}$
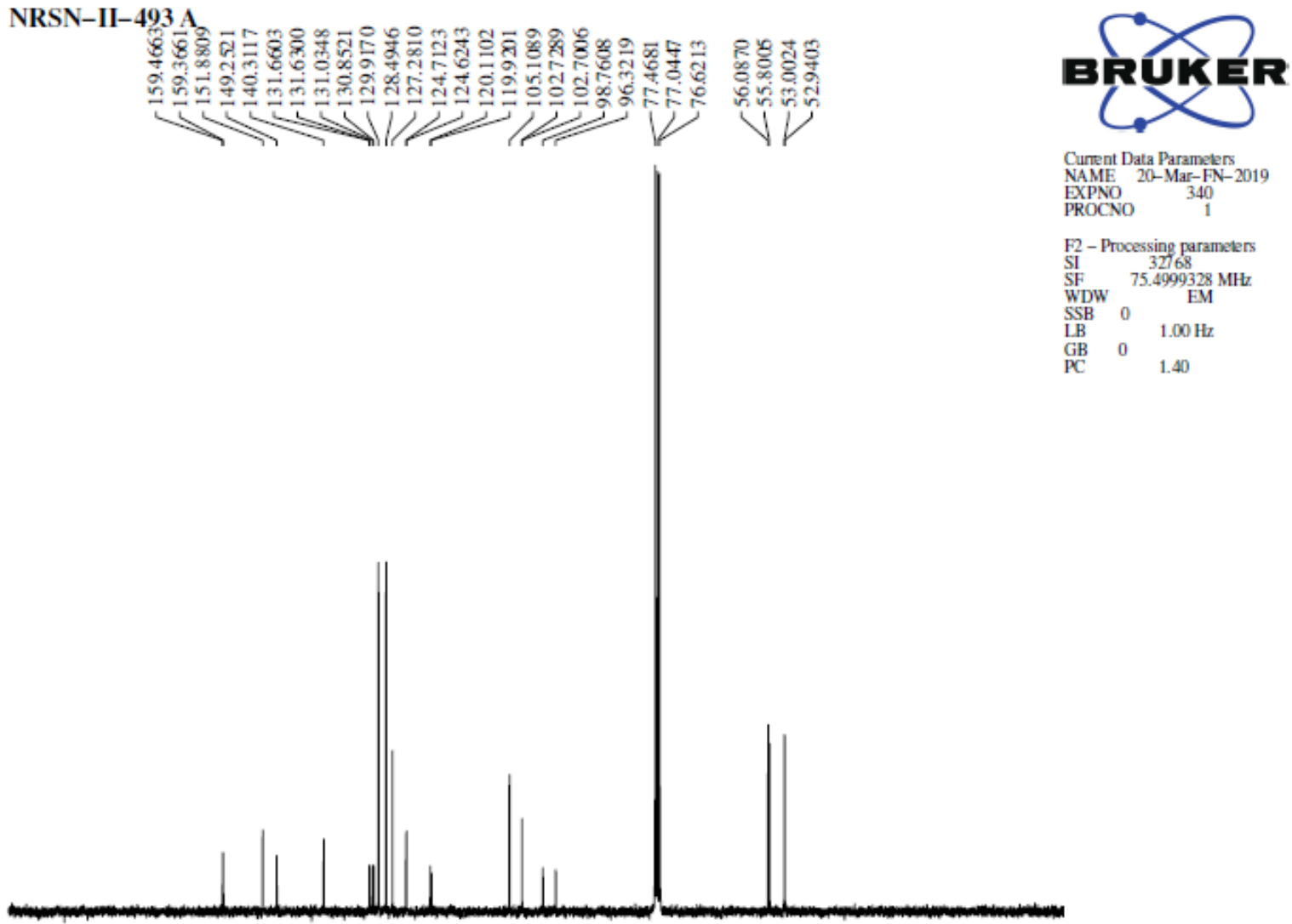

Cunent Data Parameters

NAME 20-Mar-FN-2019 PROCNO

F2 - Processing parameters

SI $\quad \begin{array}{ll}32768 \\ \text { SF }\end{array}$

DW $75.4999328 \mathrm{MM}$

$\mathrm{B}_{\mathrm{B}} \quad 0 \quad 1.00 \mathrm{~Hz}$

$\begin{array}{lll}\mathrm{GB} & 0 & 1.00 \\ \mathrm{PC} & & 1.40\end{array}$

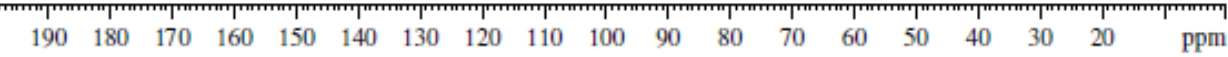

Spectrum 20: ${ }^{13} \mathrm{C}$ NMR spectrum of $\mathbf{3 g}$ 
NRSN-II-493 A

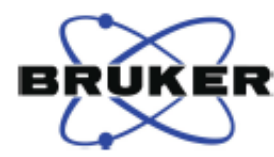

Current Data Parameters

NAME 29-Mar-AN-2019 EXPNO
PROCNO

F2 - Prucessing parameler

$\begin{array}{ll}\text { SI } & 32768 \\ \text { SF } & 121.5466600 \mathrm{MHz}\end{array}$

WDW EM

$\stackrel{S B}{L} \quad 100 \mathrm{H}$

$\stackrel{\mathrm{GB}}{\mathrm{PC}}$

$\begin{array}{ll}0 & 1.40\end{array}$

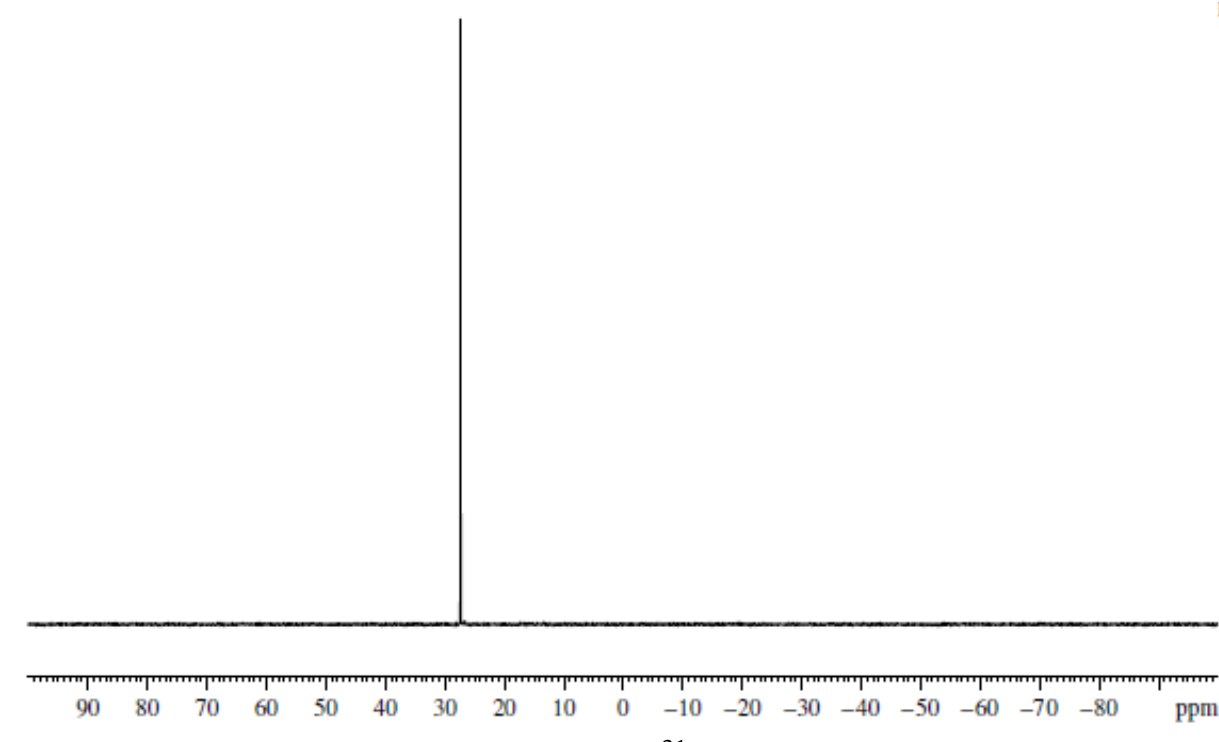

Spectrum 21: ${ }^{31} \mathrm{P}$ NMR spectrum of $\mathbf{3 g}$
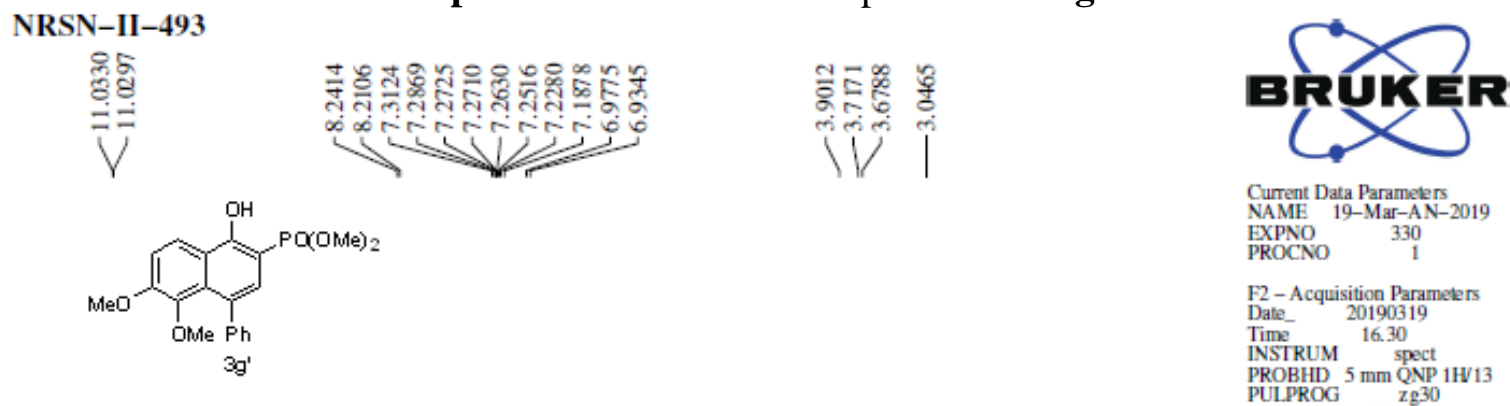

Current Data Parameters
NAME
$19-$ Mar-AN-2019 EXPNO

F2 - Acquisition Parameters

Date_ 20190319

Time 16.30

INSTRUM spect

PROBHD $5 \mathrm{~mm}$ QNP $1 \mathrm{H} / 3$

PULPROG $\quad$ Zg 30

TD $_{\text {SOLVENT }} \stackrel{65536}{\text { CDC13 }}$

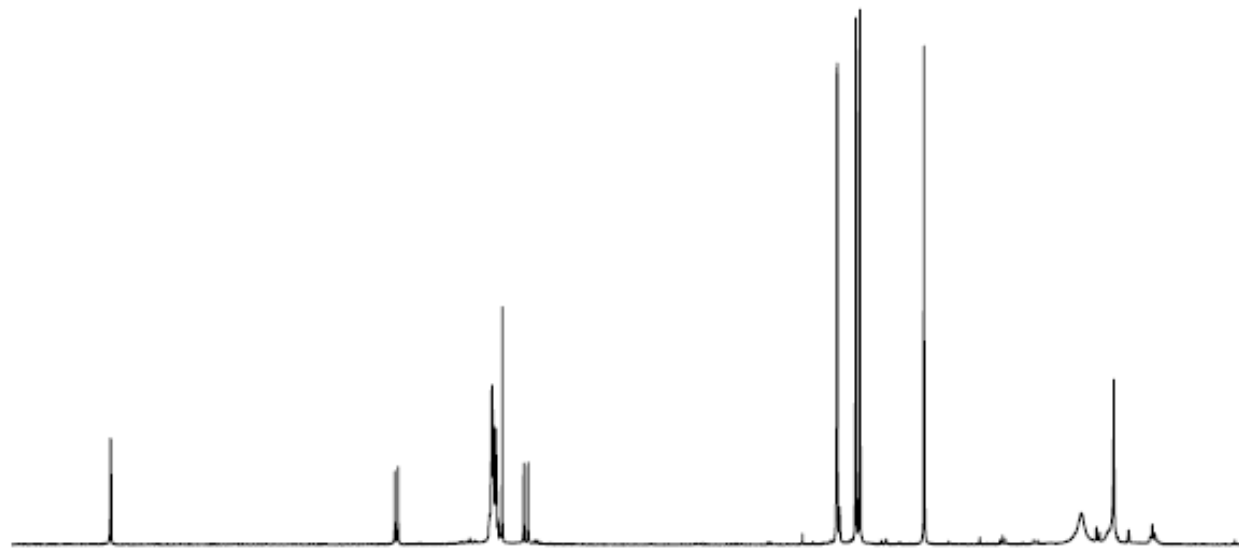

NS 16

$\begin{array}{ll}\text { DS } & 2 \\ \text { SWH } & 6188119 \mathrm{~Hz}\end{array}$

$0.094423 \mathrm{H}$
$52053096 \mathrm{sec}$

RG 256

DW $\quad 80.800$ usec

DE $\quad 3.50$ use

D1 $\quad 1.00000000 \mathrm{sec}$

TDO 1

$\overline{\mathrm{NUC1}}$ CHANNEL $\mathrm{f1}==$

13.95 usec

PL1 $\quad-1.00 \mathrm{~dB}$

SFO1 $300.2598542 \mathrm{MH}$

F2 - Processing parameters

$\begin{array}{ll}\text { SI } & 32768 \\ \text { SF } & 300.2580290 \mathrm{MH}\end{array}$

$\begin{array}{lr}\text { SF } & 300.2580290 \mathrm{M} \\ \text { WDW } & \mathrm{EM}\end{array}$

SSB 0

$\begin{array}{lll}\text { L.B } & 0.30 \mathrm{H} \\ \text { GB } & 0 & \end{array}$

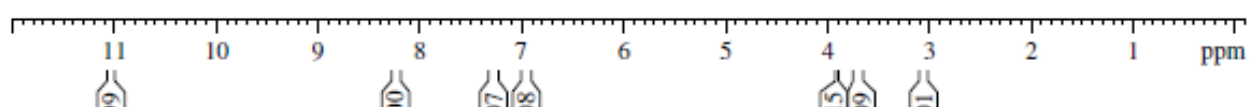

g.

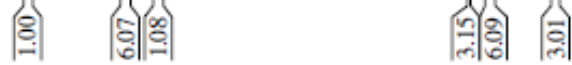

Spectrum 22: ${ }^{1} \mathrm{H}$ NMR spectrum of $\mathbf{3 g}$ ' 


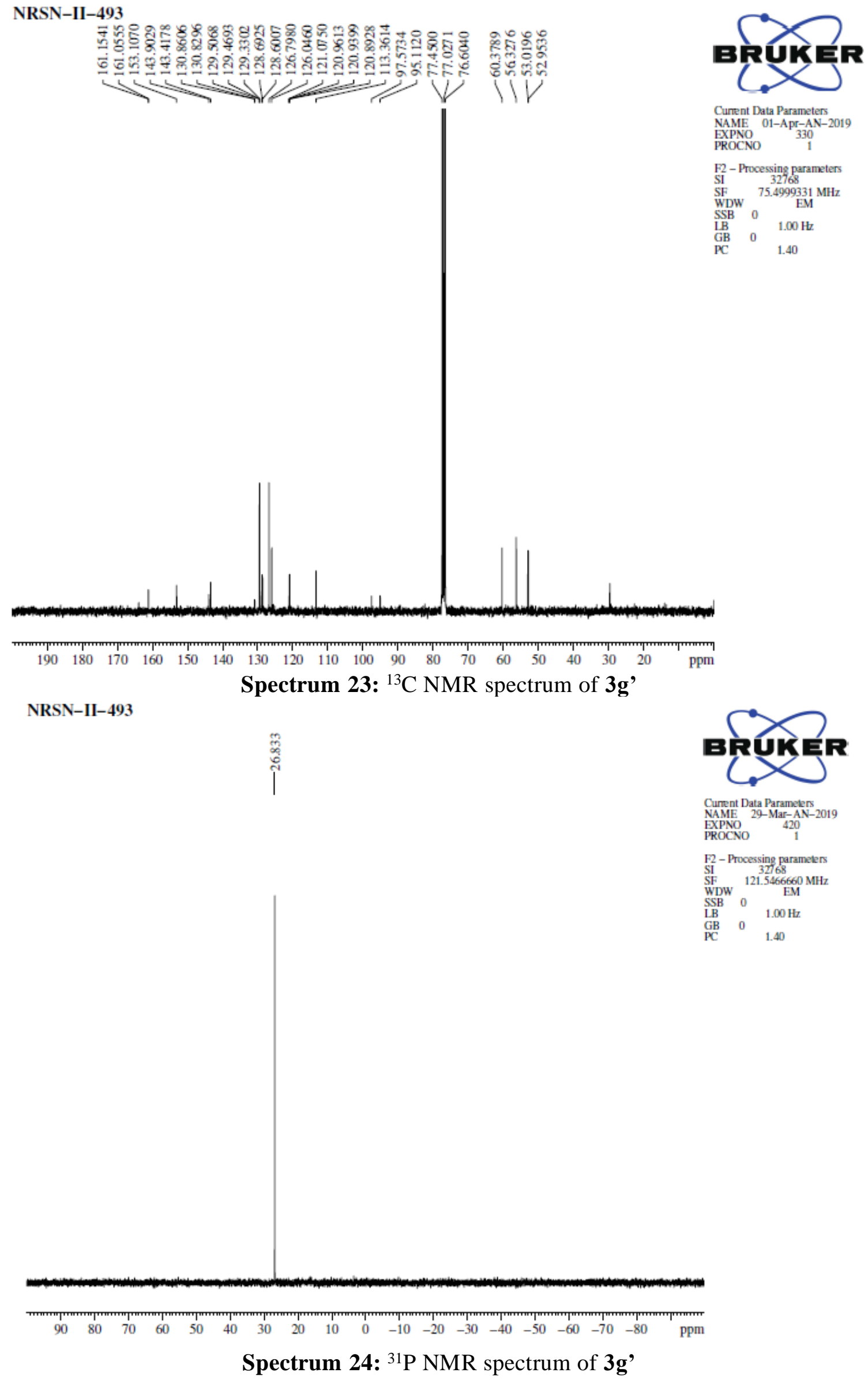


NRSN II 395 A

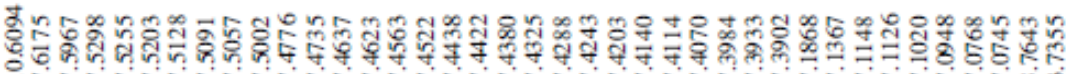

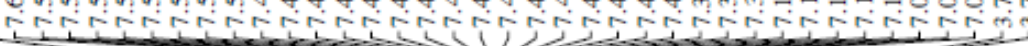
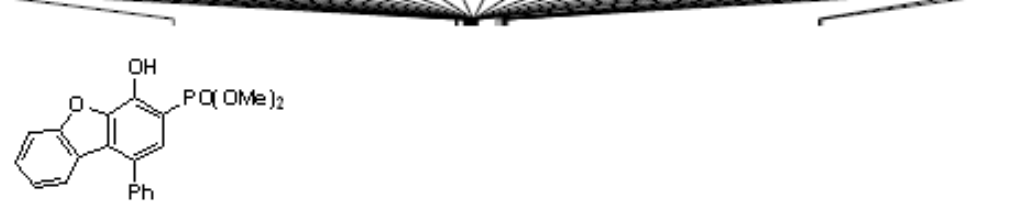

TUKER

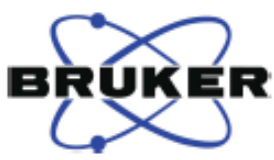

Cuntent Data Parameters 2018 EXPNO

F2 - Acquisition Parameters

Date_ 20180322

Time 23.39

INSTRUM PROCt

औ

PULPROG 2030

TD ${ }_{\text {SOLVENT }}^{65536}$ CDCB

$\begin{array}{ll}\text { NS } & 8 \\ \text { DS } & 8\end{array}$

SWH $\quad 9615.385 \mathrm{~Hz}$

FIDRES $0.146719 \mathrm{H}$

AQ $\quad 3.4078720 \mathrm{sec}$

$\begin{array}{ll}\text { RG } & 129.57 \\ \text { DW } & 52000 \text { usec }\end{array}$

$\begin{array}{ll}\text { DE } & 6.50 \mathrm{usec} \\ \text { TE } & 300.0 \mathrm{~K}\end{array}$

D1 $1.00000000 \mathrm{sec}$
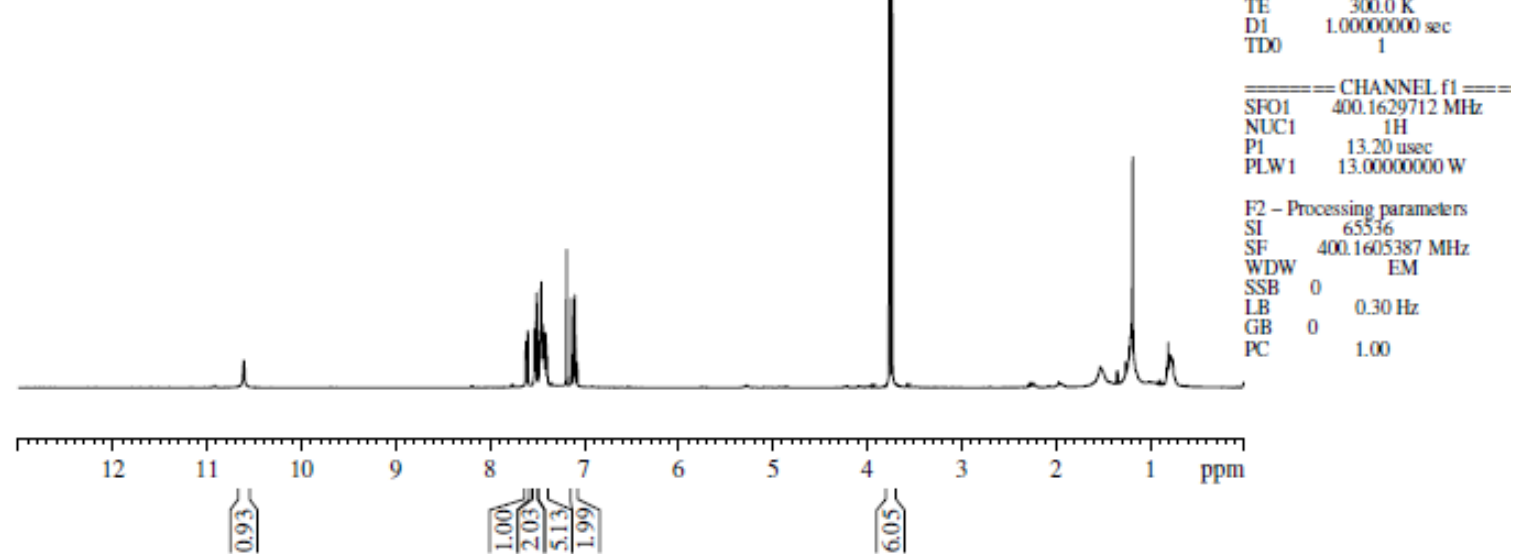

Spectrum 25: ${ }^{1} \mathrm{H}$ NMR spectrum of $\mathbf{3 h}$
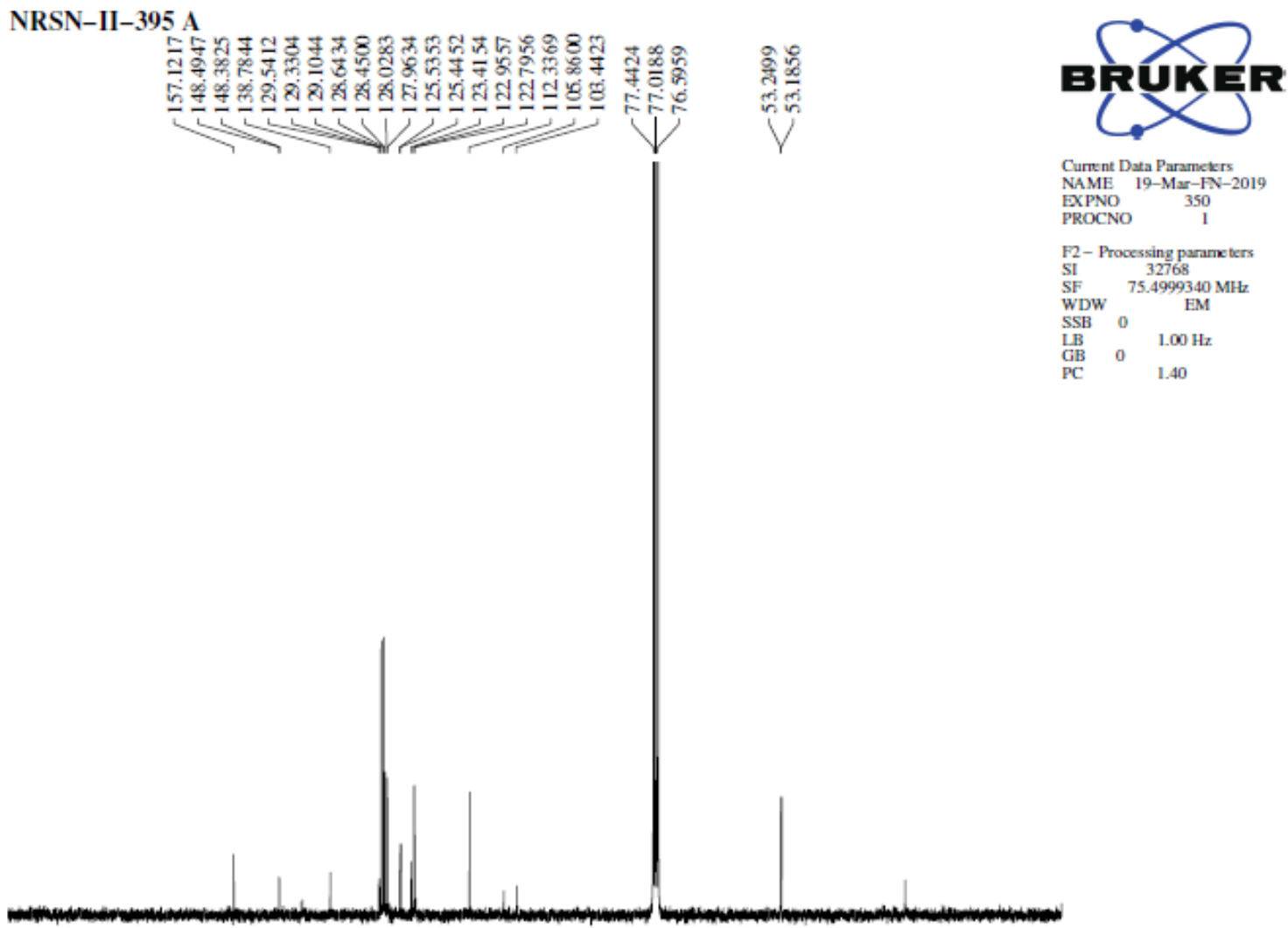
Current Data Parameters EXPNO 350

F2 - Processing parane ters

32768
$\mathrm{SF}$ WDW EM SSB 0 $\underset{\mathrm{GB}}{\mathrm{LB}} 0.1 .00 \mathrm{~Hz}$ 


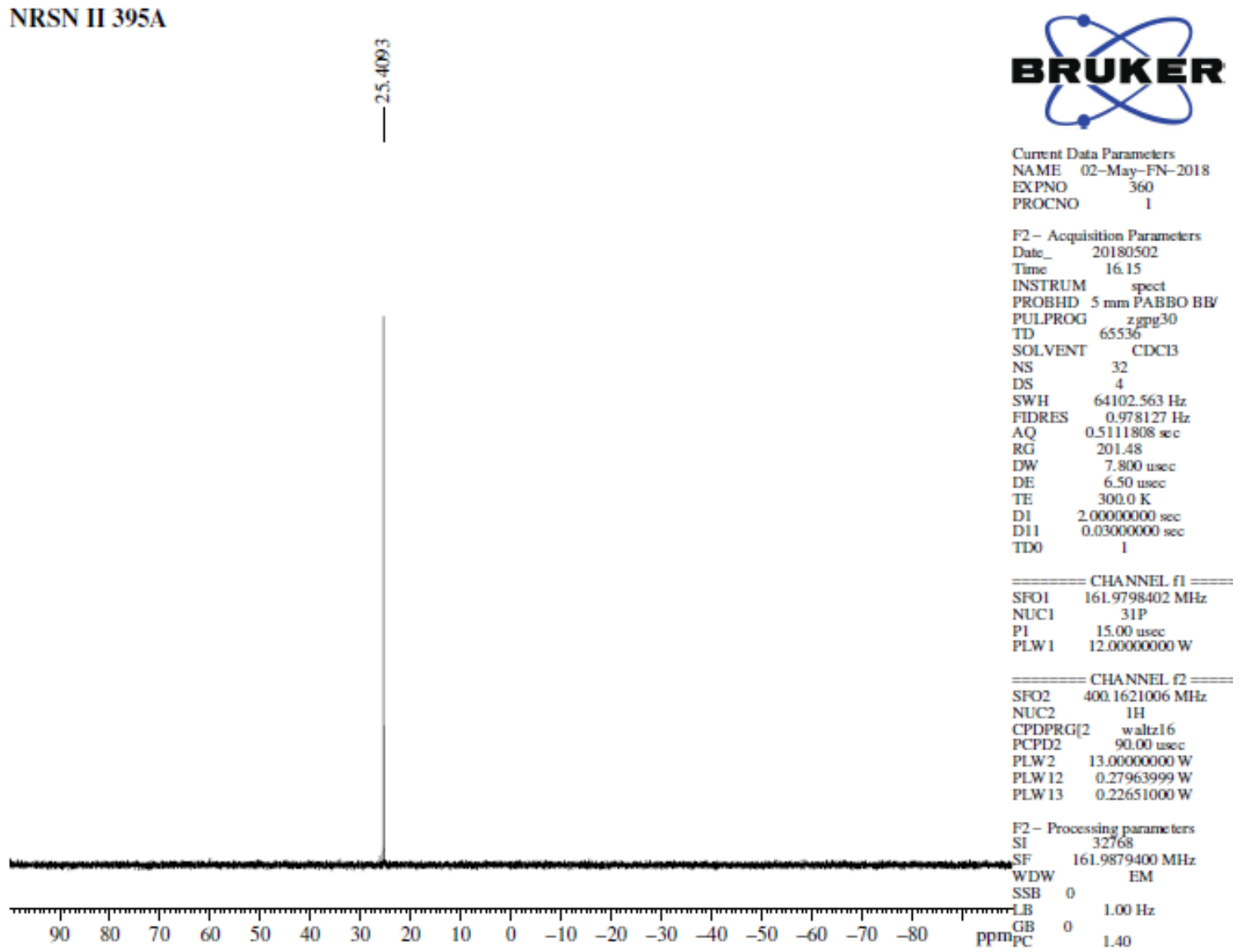

Spectrum 27: ${ }^{31} \mathrm{P}$ NMR spectrum of $\mathbf{3 h}$

NRSN II 399
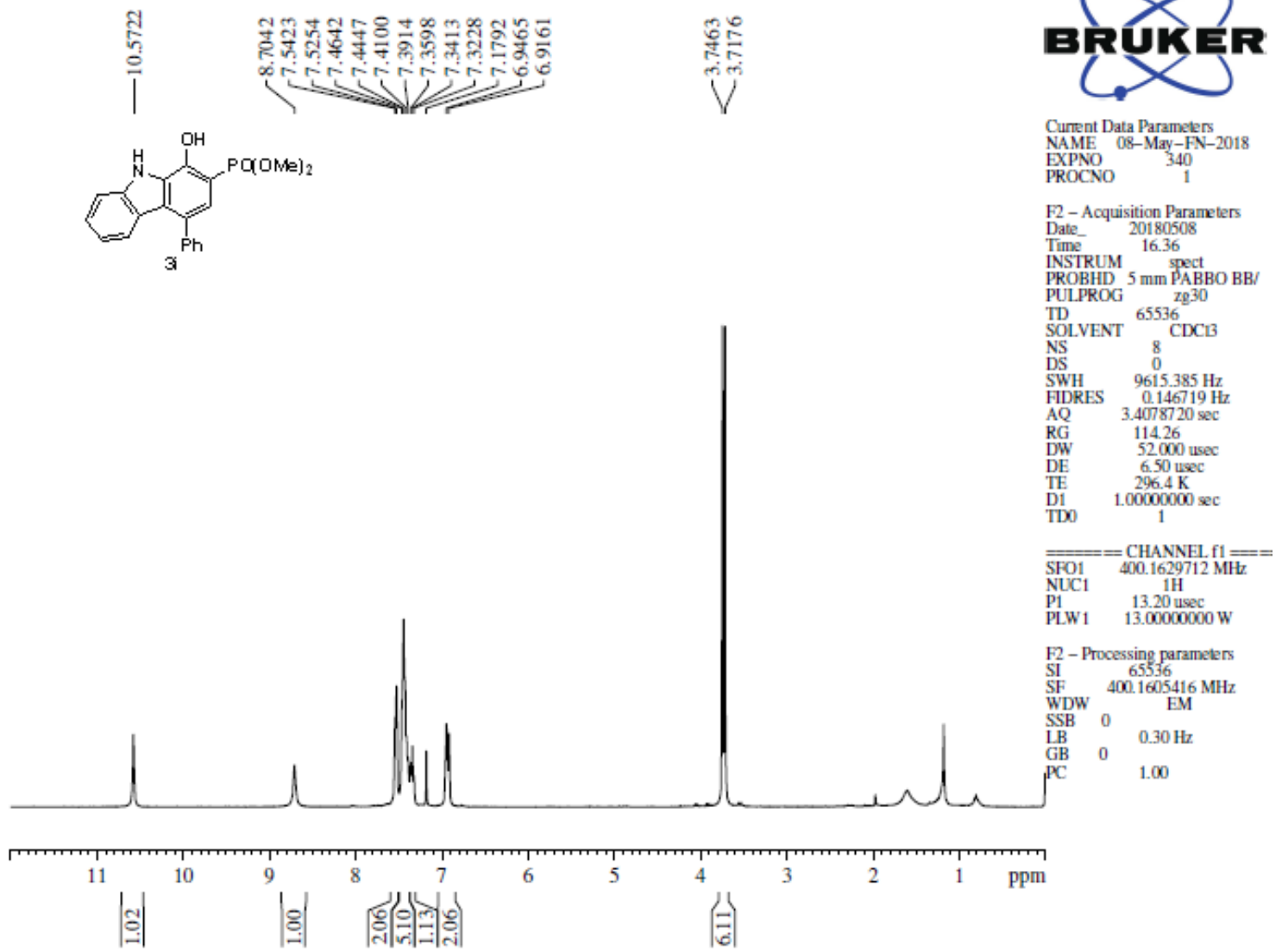

Spectrum 28: ${ }^{1} \mathrm{H}$ NMR spectrum of $\mathbf{3 i}$ 


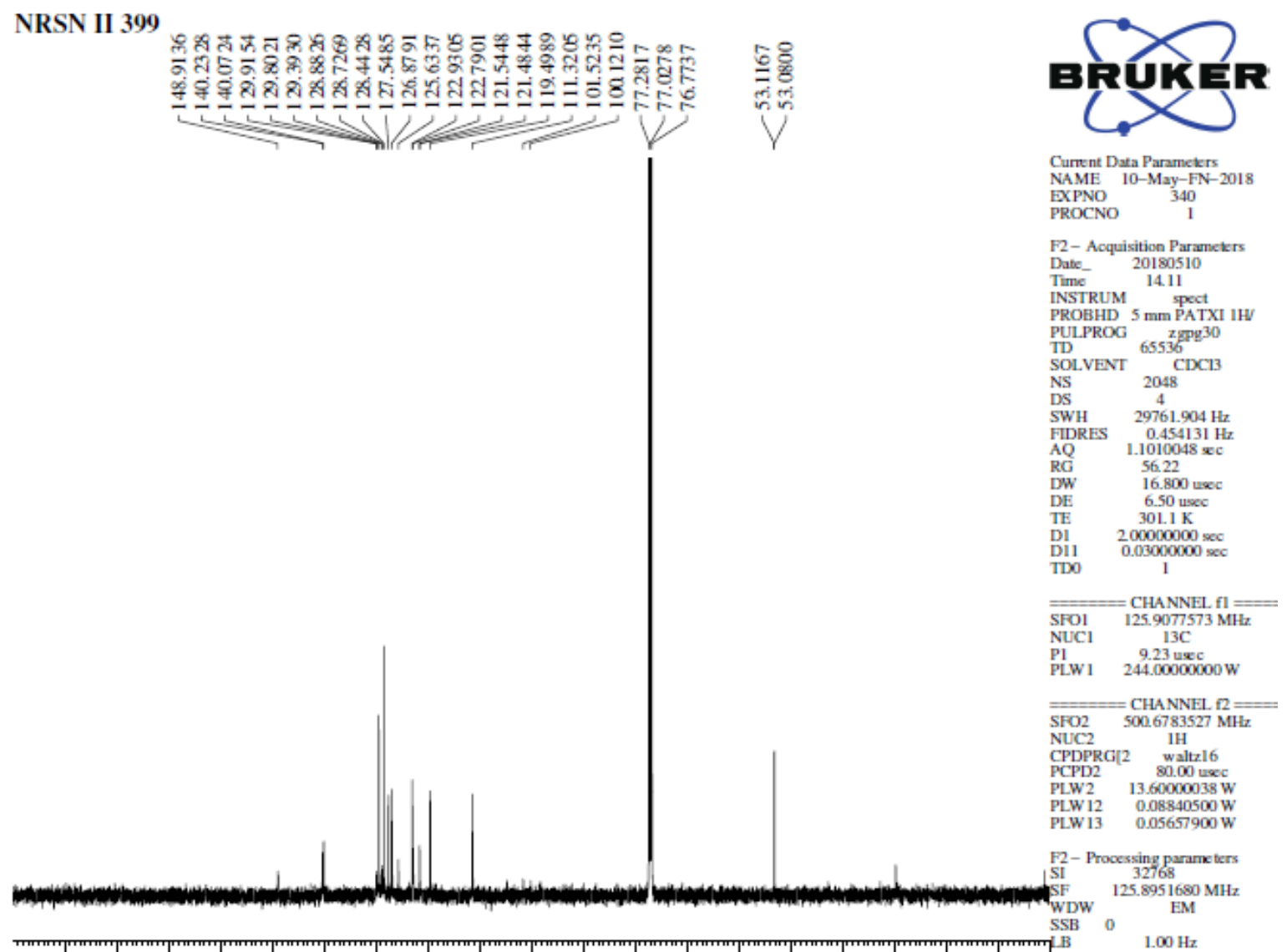

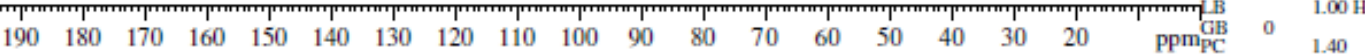

Spectrum 29: ${ }^{13} \mathrm{C}$ NMR spectrum of $3 \mathbf{i}$

NRSN II 390

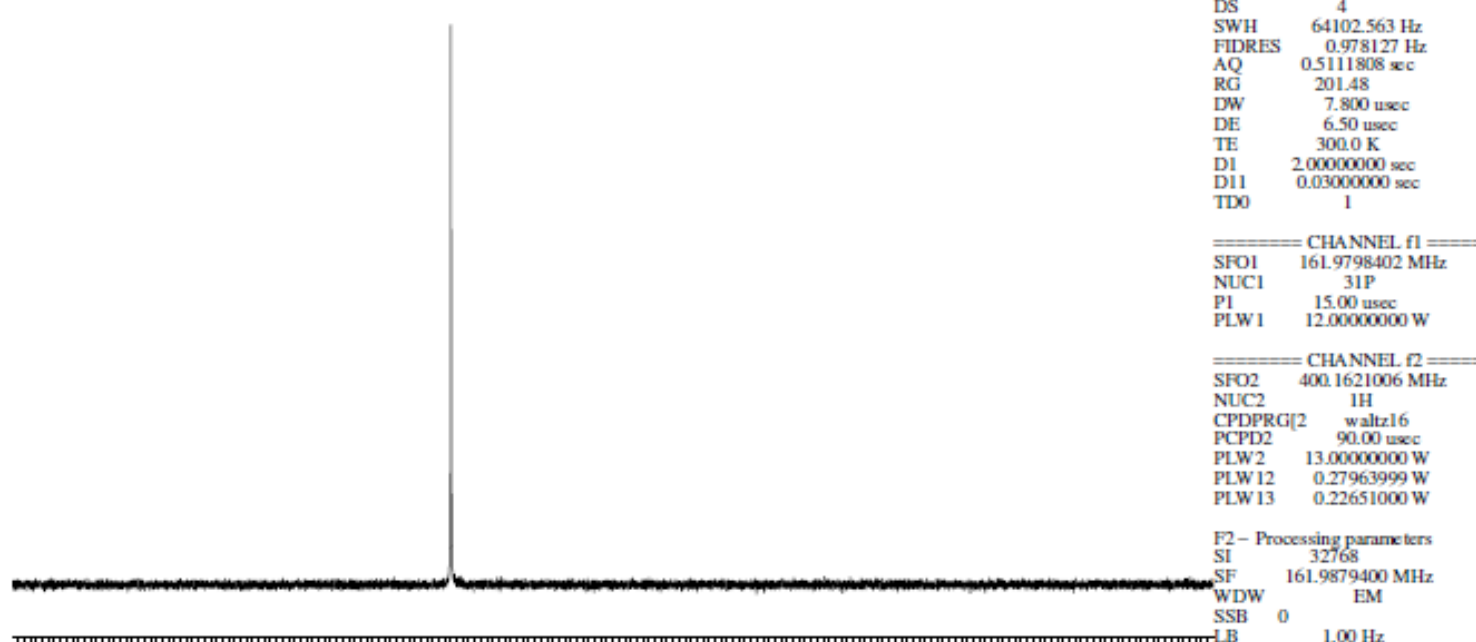

BRUKER

Current Data Parameters NAME 14-May-2018-F EXPNO

F2- Acquisition Parameters

Date_- 20180514

Time 14.59

INSTRUM $\begin{gathered}\text { spet } \\ \text { PROBHD } 5 \text { mm PABBO BBy }\end{gathered}$

PULPROG $25 \mathrm{gpg}^{30}$

TD
SOLVENT $\quad{ }^{65536}$ CDC13

SOLVENT
NS

ps

$0.97863 \mathrm{H}_{2}$

0.5111808 xec

201.48

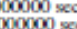

C CHANEL $f 1=$

FFol $161.9798402 \mathrm{MP}$

\begin{tabular}{lrl} 
NUCl & $31 P$ \\
\hline 1 & 15.00
\end{tabular}

$12.00000000 \mathrm{~W}$

$\begin{array}{ll}\mathrm{SFO} 2 & 402.1621006 \mathrm{MHz}\end{array}$



PCPD2 90.00 used

$0.27963999 \mathrm{~W}$

S1- Processing paranne ters $5 \mathrm{SB} \quad 0-2$

$\begin{array}{llllllllllllllllllllllll}90 & 80 & 70 & 60 & 50 & 40 & 30 & 20 & 10 & 0 & -10 & -20 & -30 & -40 & -50 & -60 & -70 & -80 & \mathrm{ppm} & \mathrm{GB} & 0 & 1.40\end{array}$

Spectrum 30: ${ }^{31} \mathrm{P}$ NMR spectrum of $3 \mathbf{i}$ 


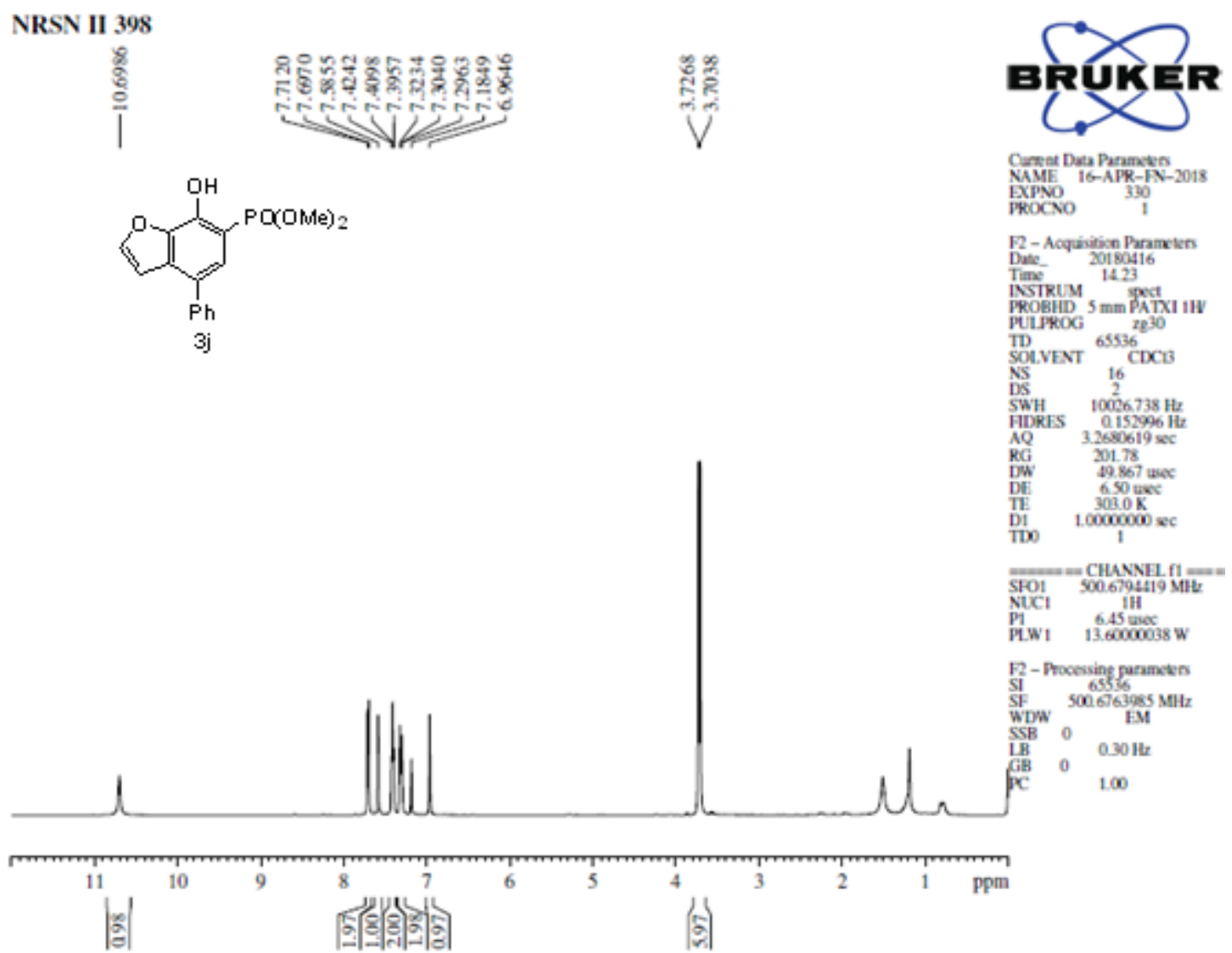

Spectrum 31: ${ }^{1} \mathrm{H}$ NMR spectrum of $\mathbf{3 j}$

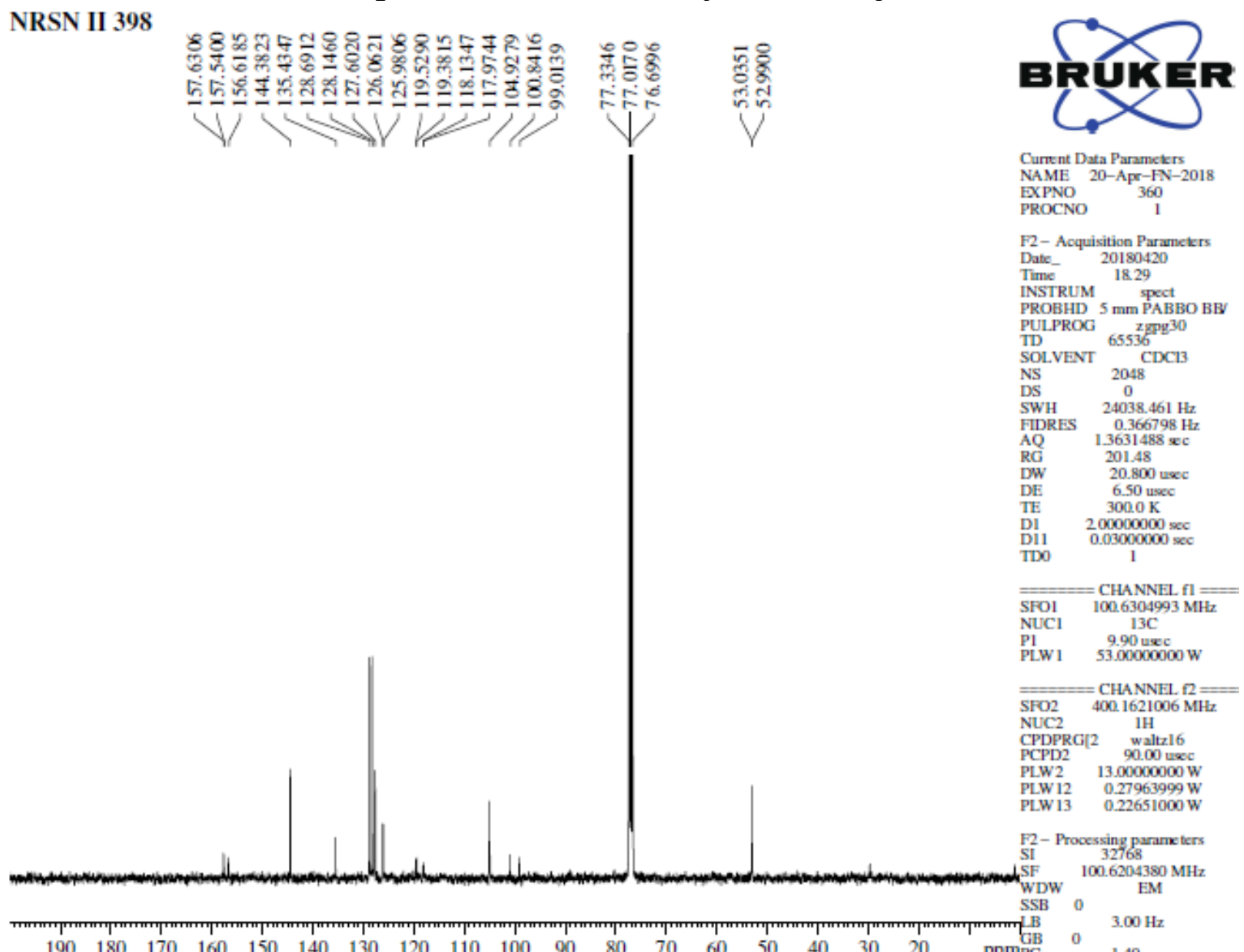

Spectrum 32: ${ }^{13} \mathrm{C}$ NMR spectrum of $\mathbf{3 j}$ 


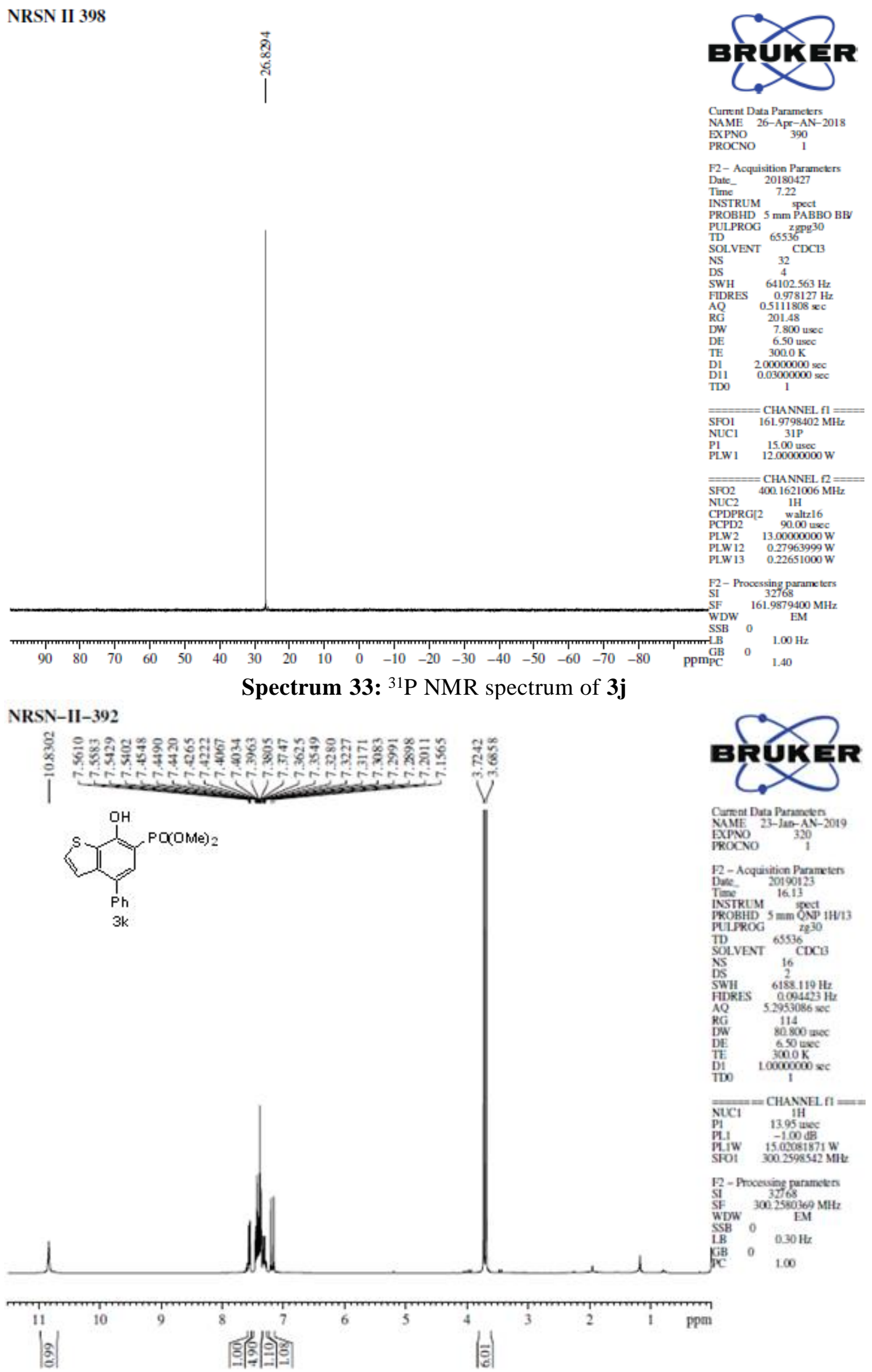

Spectrum 34: ${ }^{1} \mathrm{H}$ NMR spectrum of $\mathbf{3 k}$ 

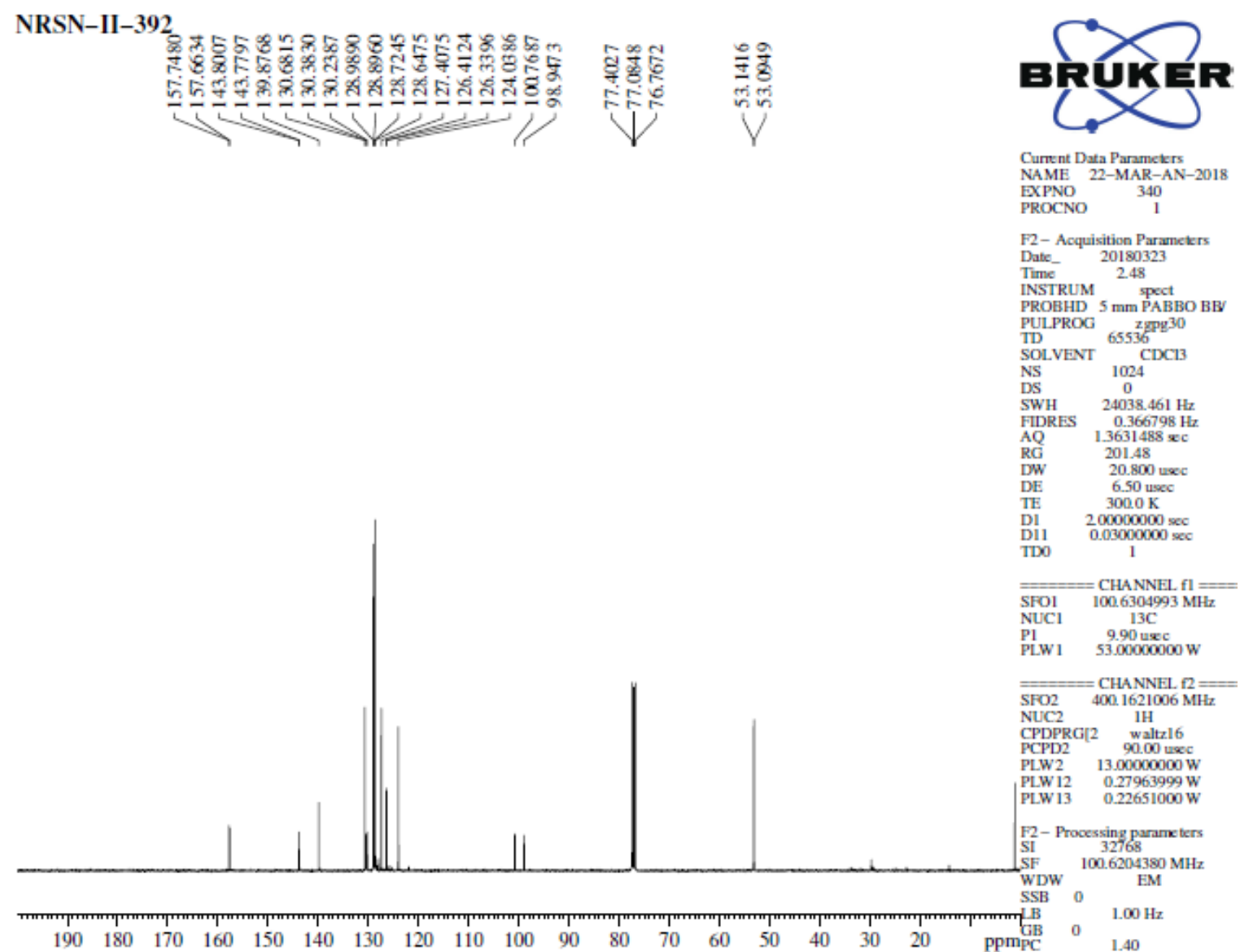

Spectrum 35: ${ }^{13} \mathrm{C}$ NMR spectrum of $\mathbf{3 k}$

NRSN-II-392
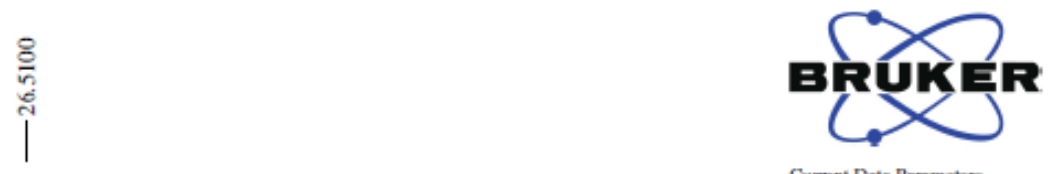

Curnent Data Parameters

NAME 23-Mar-AN-

EXPNO

F2- Acquisition Parameters

Date-

21.50

INSTRUM
PROBHD 5 mm PABE
PABO BB

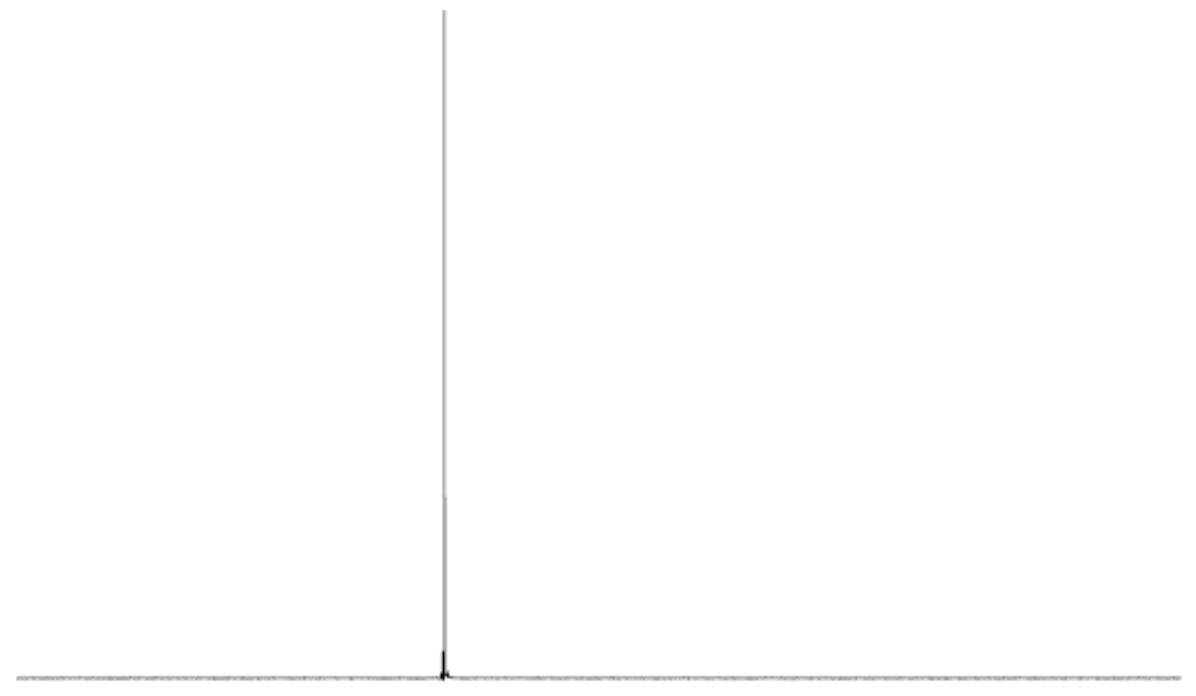

TD $6553 \%$. $39 \mathrm{~g}$

SOLVENT CDCI

NS $\quad 16$

DSH $\quad 44102563 \mathrm{~Hz}$

FIDRES $\quad 0.978127 \mathrm{H}$

AQ 0.5111808

$\begin{array}{ll}\text { RG } & 201.48 \\ \text { DW } & 7.800 \text { usec }\end{array}$

TE $\quad 3002$ K

DI $\quad 200000000 \mathrm{sec}$

DI1 0.03000000

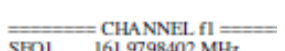

SFOCl 161.9798 .02

$\begin{array}{ll}\text { Pl } & 15.00 \text { usec } \\ \text { PLW1 } & 12.00000000 \mathrm{~W}\end{array}$

$=$ CHANNEL $\mathrm{F} 2=$

$\mathrm{N} F 224021621 \mathrm{H}$

CPDPRRG12
waltz16
90.00 ueed

$\begin{array}{ll}\text { PCPD2 } & 90.00 \text { usec } \\ \text { PLW2 } & 13.00000000 \mathrm{~W}\end{array}$

PLW 12 $0.27963999 \mathrm{~W}$

F2- Processing parane ters

SF $\quad 161.9879400 \mathrm{MHz}$

WDW EM

$\begin{array}{lll}\mathrm{SSB} & 0 & 1.00 \mathrm{~Hz}\end{array}$

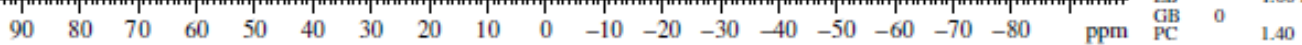

Spectrum 36: ${ }^{31} \mathrm{P}$ NMR spectrum of $\mathbf{3 k}$ 


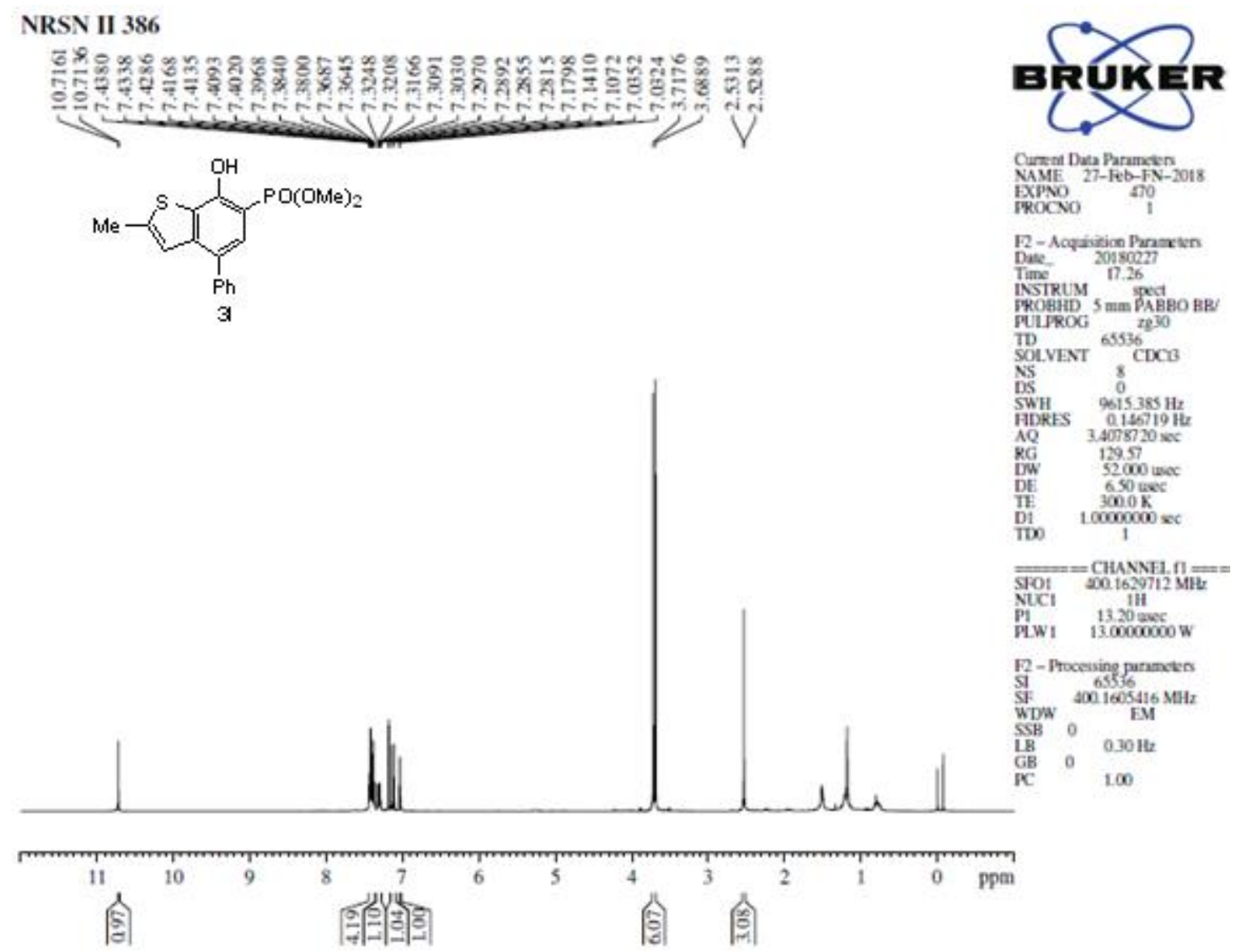

Spectrum 37: ${ }^{1} \mathrm{H}$ NMR spectrum of 31

NRSN-II-386

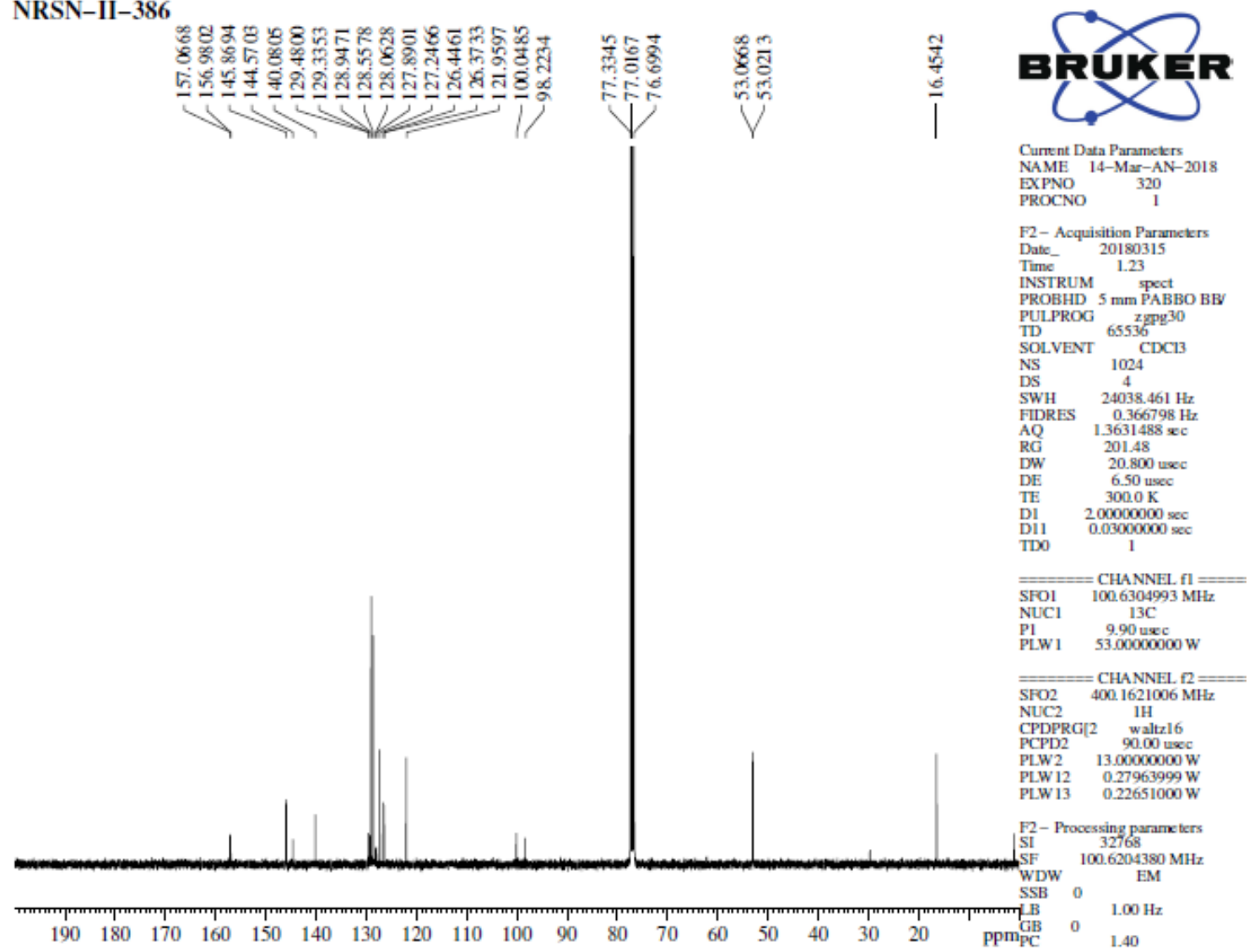

Spectrum 38: ${ }^{13} \mathrm{C}$ NMR spectrum of 31 
NRSN-II-386

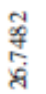

añun

Current Data Paramekers
NAME 16-Mar-AN-2018

EXPNO

PROCNO

1

F2- Acquisition Parameters

Date_ 20190317

Time

9.07

PROBHD 5 mm PABBO BB

PULPROG $2 \mathrm{gPg} 30$

SOLVENT $\quad$ CDC73

$\begin{array}{ll}\text { NS } & 16 \\ \text { DS } & 4\end{array}$

$\begin{array}{ll}\text { DS } & 4 \\ \text { SWH } & 64102.563 \mathrm{~Hz}\end{array}$

$\begin{array}{lr}\text { SWDRES } & 64102.563 \mathrm{~Hz} \\ \text { FIDRE } & 0.978127 \mathrm{H}\end{array}$

AQ 0.5111808 se

DW $\quad 7.800 \mathrm{usec}$

TE $\quad 3002 \mathrm{~K}$

$\begin{array}{ll}\text { DI } & 2000000000 \mathrm{sec} \\ \text { DI1 } & 0.030000000 \mathrm{sec}\end{array}$

CHANNEL $\mathrm{fl}=$

NUC1 $\quad 31 \mathrm{P}$

$\begin{array}{ll}\text { P1 } & 15.00 \text { usec } \\ \text { PLW1 } 12.00000000 \mathrm{~W}\end{array}$

CHANNEL $\cap 2=$

SFO2 $400.1621006 \mathrm{MF}$

NUC2 $1 \mathrm{H}$

PCPD2 2 waltz16

PLW2 $13.00000000 \mathrm{~W}$

PLW $120.27963999 \mathrm{~W}$

PLW13 0.2265000 W

Fi- Processing parame ters $\begin{array}{lc}\text { SI } & 32768 \\ \text { WDW } & 161.9879400 \mathrm{MHz}\end{array}$

SSB 0 EM

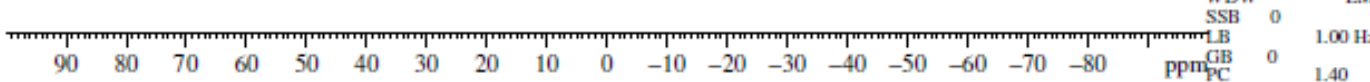

Spectrum 39: ${ }^{31} \mathrm{P}$ NMR spectrum of 31

NRSN II 406

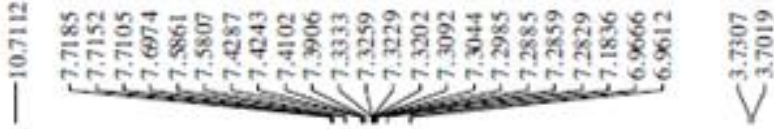
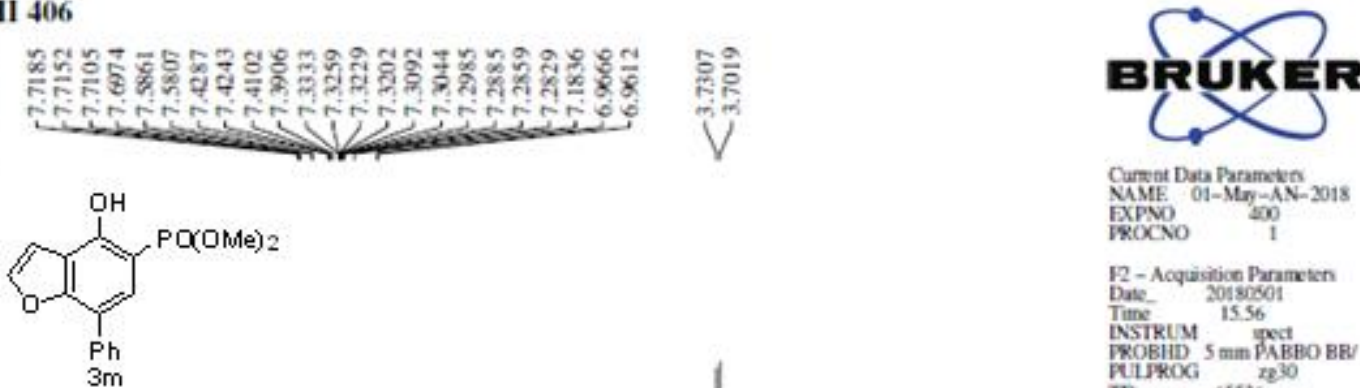

Cursent Dasa Parametes
XME. 01-Mry-AN-2018

EXPNo

F2-Acquistion Parameten

Date 2018060 t

Tine 1556

WOBHD $5 \mathrm{~mm}$ PABRO BE

PULFOG te

SOLVENT 65536 CDC2

NS

ธS $9615335 \mathrm{~Hz}$

SWH
HDRIS $\quad 015365 \mathrm{Ht}$

HORES $3016719 \%$

AQ $\quad 3,40053$

DW $\quad 52000$ we

正

Dit

$300 \mathrm{~K}$

10000000

1

STOt $20162 \mathrm{~V} 712 \mathrm{Mth}$

SuCl 111

P1 1320 1.w1 $130000000 \mathrm{w}$
niw1

P2-Procesing parameten

if $400.1605395 \mathrm{Mtt}$

WDW 400.1000

$\begin{array}{lll}\text { SSB } & 0 \\ \text { IB } & 0.30 \mathrm{H}\end{array}$

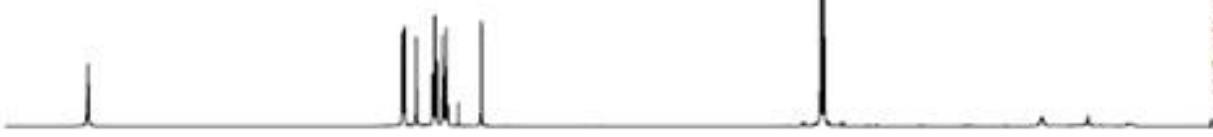

1.00

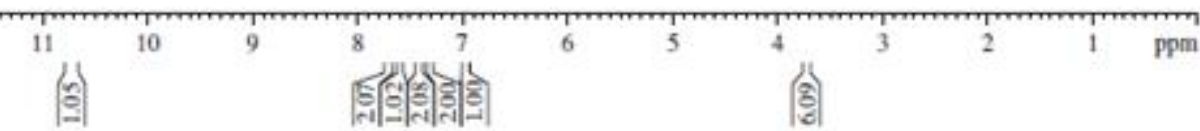

Spectrum 40: ${ }^{1} \mathrm{H}$ NMR spectrum of $\mathbf{3 m}$ 


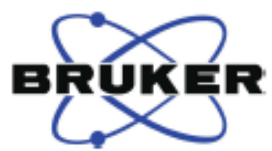

Curnent Data Parameters

NAME 03-May-FN-2018 EXPNO
PROCNO

F2- Acquisition Paramelers

Time- 11.58

PROBHD 5 mm PATXI IH

PULPROG $25 \mathrm{zPg}^{30}$

TD $6553 \%$

NS 204

DS

SWH $\quad 29761.904 \mathrm{~Hz}$

FDRES $0.454131 \mathrm{~Hz}$

RG 81.53

$\begin{array}{ll}\mathrm{DW} & 16.800 \text { used } \\ \mathrm{DE} & 6.50 \text { usec }\end{array}$

TE $3008 \mathrm{~K}$

$\begin{array}{ll}\text { DI } & 200000000 \mathrm{sec} \\ \text { DII } & 0.03000000 \mathrm{sec}\end{array}$

$\begin{array}{lr}\text { DI1 } & 0.0300000 \\ \text { TD } & 1\end{array}$

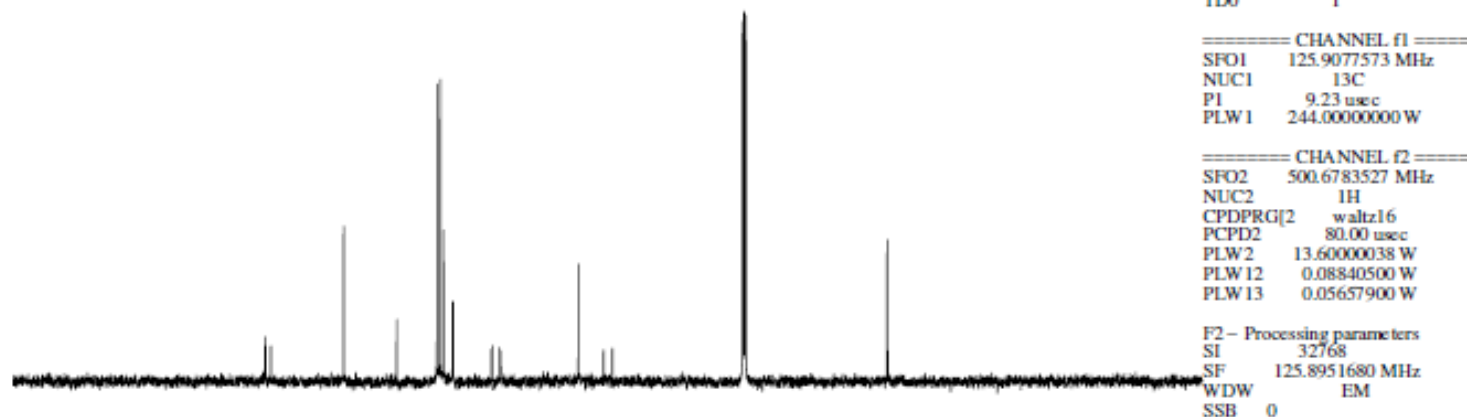

SSB 0 EM

$\begin{array}{lllllllllllllllllllll}190 & 180 & 170 & 160 & 150 & 140 & 130 & 120 & 110 & 100 & 90 & 80 & 70 & 60 & 50 & 40 & 30 & 20 & \text { PPmPC } & 0 & 1.40\end{array}$

NRSN II 406

Spectrum 41: ${ }^{13} \mathrm{C}$ NMR spectrum of $3 \mathbf{m}$

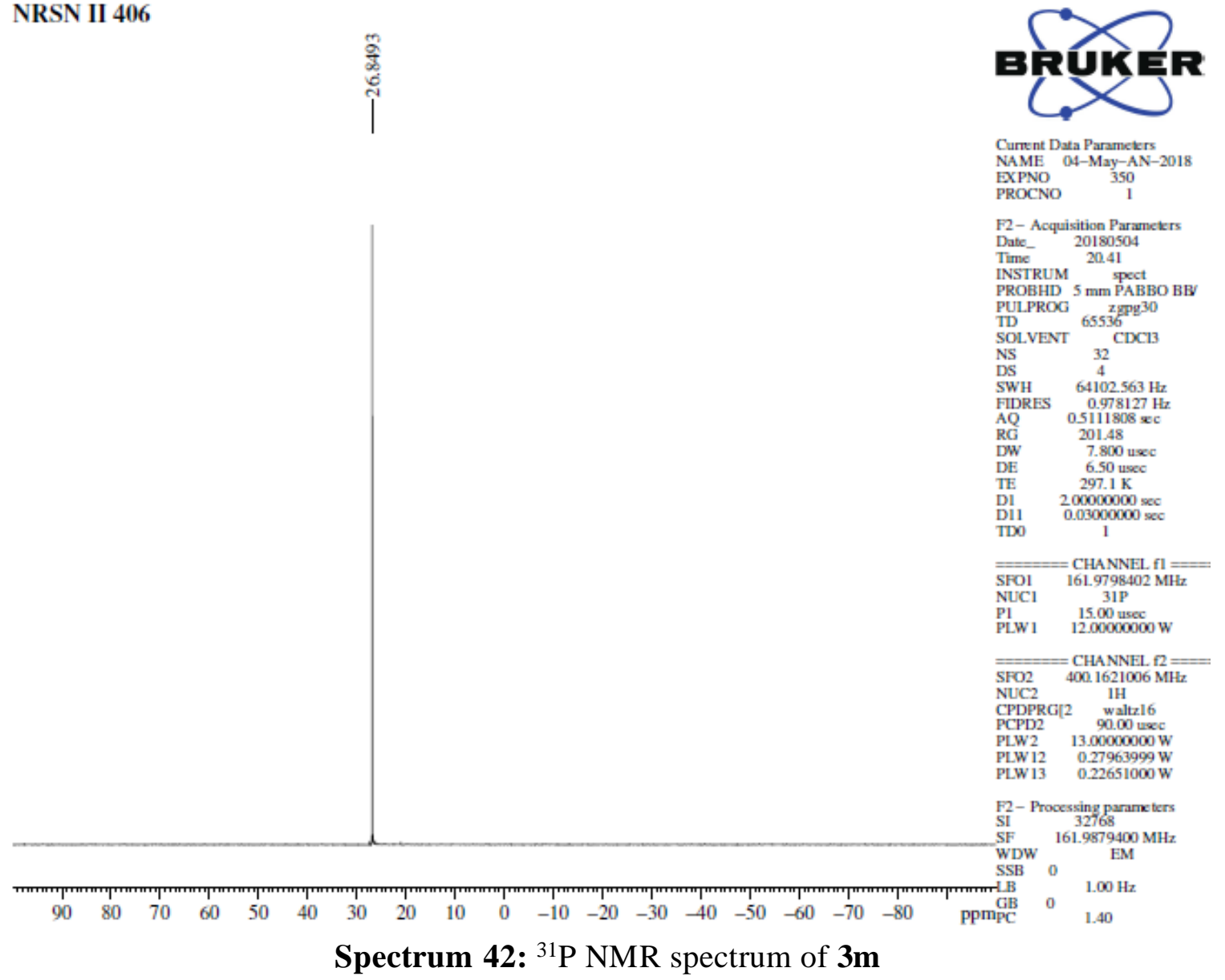




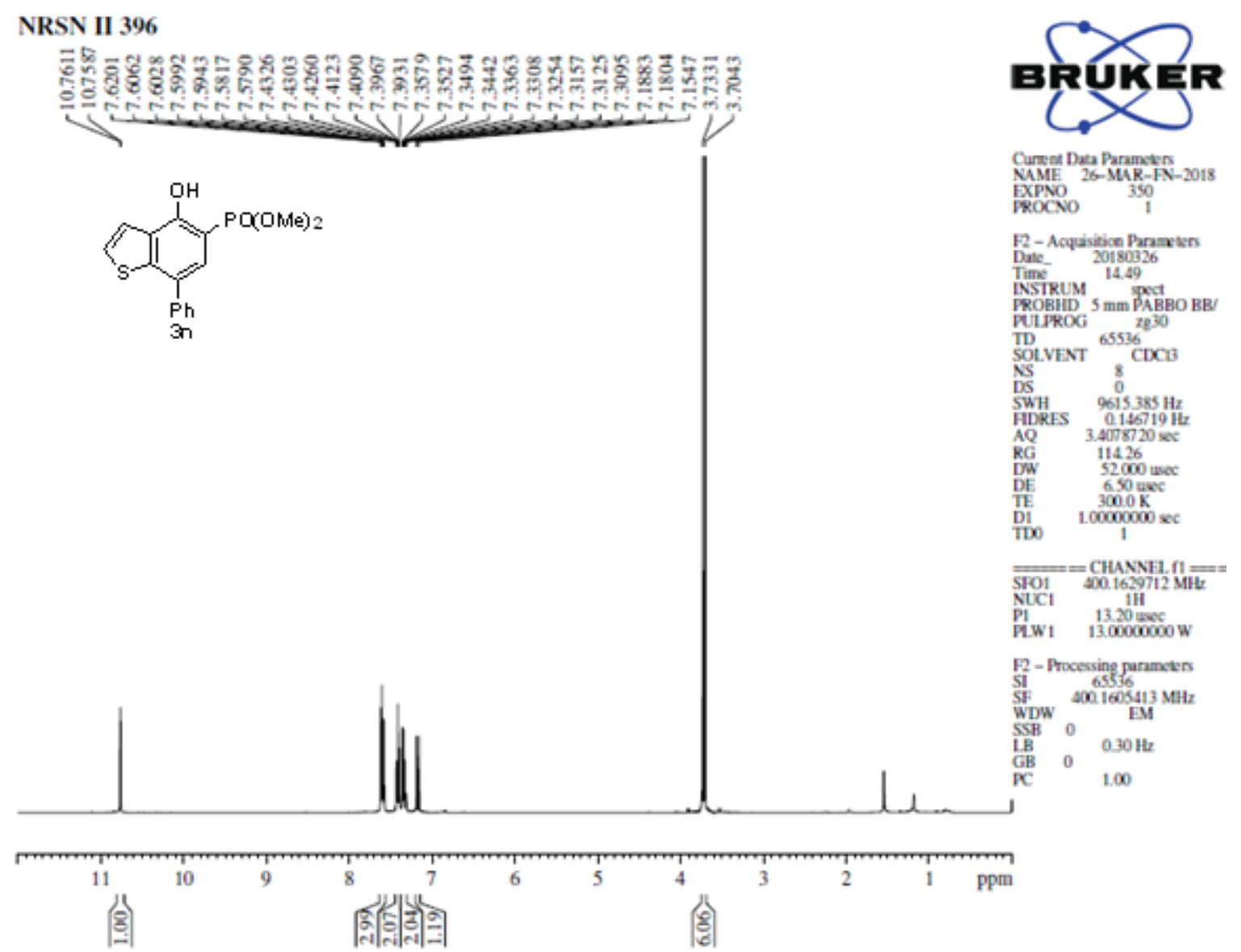

Spectrum 43: ${ }^{1} \mathrm{H}$ NMR spectrum of $3 \mathbf{n}$

NRSN-II 396
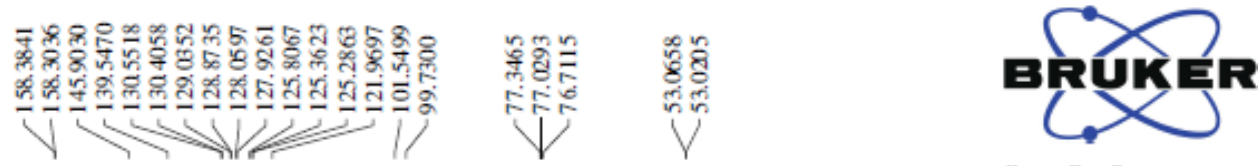

Current Data Parameters

EXPNO 310

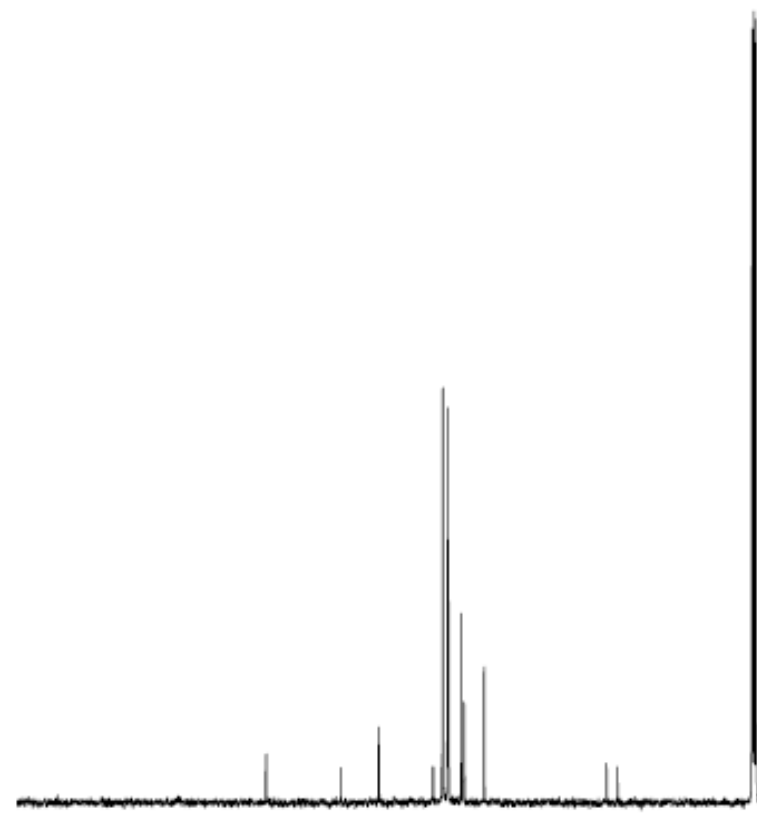

F2-Acquisition Parameters
Date- 20180327

Date_ 20180327 16.41

PROBHD 5 mm PABBO BB

PULPROG 2 Ppg 30

ID 65536 .

SOLVENT CDC

NS $\quad 102$

$\begin{array}{lll}\text { DS } & 0 \\ \text { SWH } & 24038.461 \mathrm{~Hz}\end{array}$

FIDRES $\begin{aligned} 0.366798 \mathrm{~Hz} \\ 1.3631488 \mathrm{xc}\end{aligned}$

$\begin{array}{ll}\mathrm{AQ} & 1.3631488 \times \\ \mathrm{RG} & 201.48\end{array}$

\begin{tabular}{ll}
$R G$ & 201.48 \\
DW & 20.800 \\
\hline
\end{tabular}

DE $\quad 6.50$ usec

200000000

$\begin{array}{lc}\text { Dl } & 200000000 \mathrm{sec} \\ \text { DI1 } & 0.03000000 \mathrm{sec}\end{array}$

TDO

CHANNEL $\mathrm{fl}=$

SFOI 100.6304993 M.

990

$\begin{array}{ll}\text { PI } & 9.90000 \mathrm{c} \\ \text { PLW1 } 53.00000000 \mathrm{~W}\end{array}$

$=$ CHANNEL $R=$

SFO2 $400.1621006 \mathrm{M}$

CPDPRG12 1H

CPDPRG12 waltz16

PLW2 $13.00000000 \mathrm{~W}$

PLW $120.27963999 \mathrm{~W}$

PLW $13-0.22651000 \mathrm{~W}$

F2- Processing parame ters
SI

SF $\quad 100.6204380 \mathrm{MHz}$

$\begin{array}{lll}\mathrm{SSB} & 0 & \\ \mathrm{~B} & & \end{array} .00 \mathrm{~Hz}$

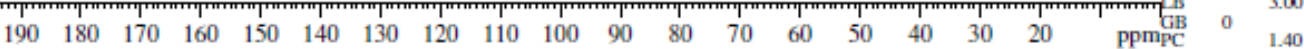

Spectrum 44: ${ }^{13} \mathrm{C}$ NMR spectrum of $3 n$ 


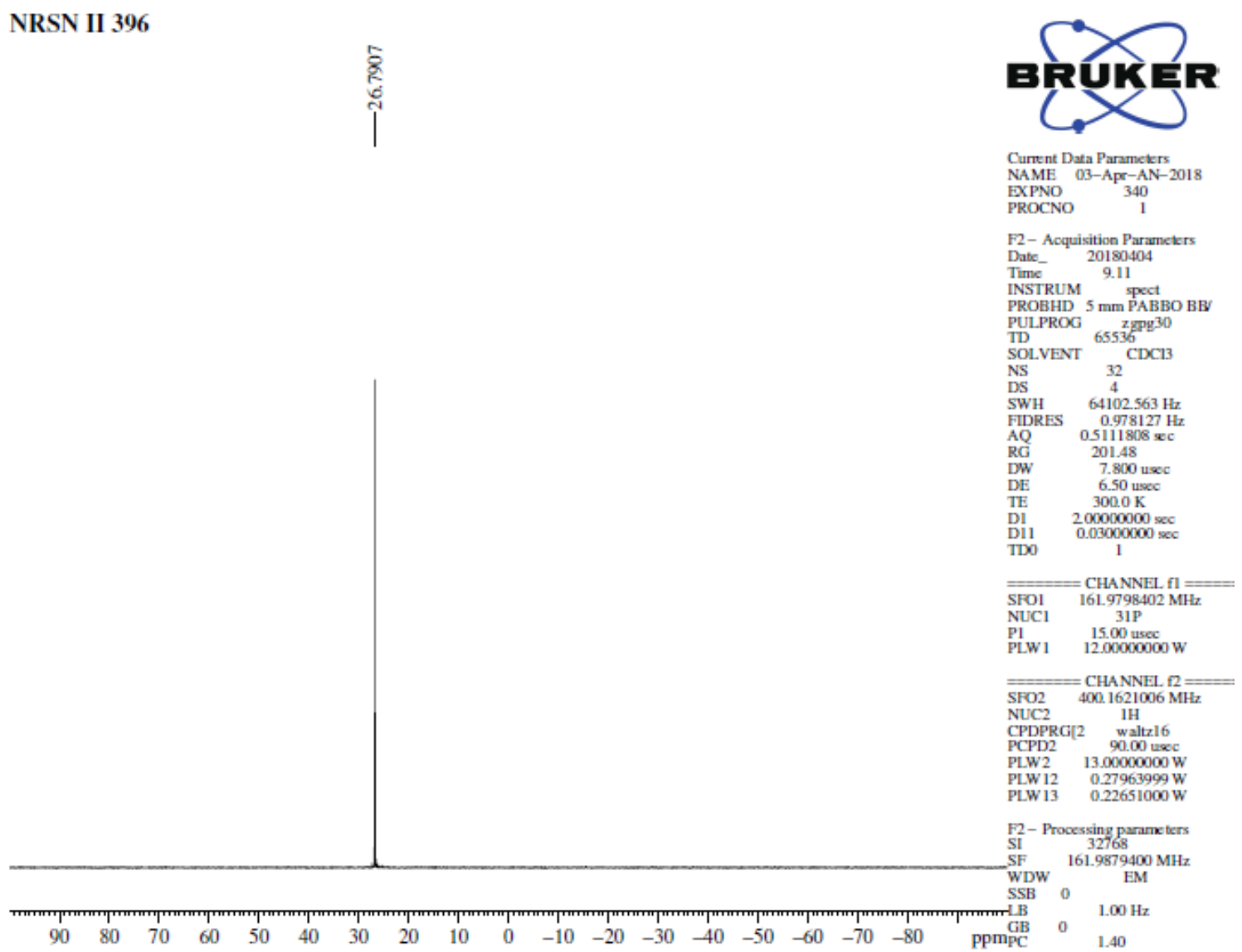

Spectrum 45: ${ }^{31} \mathrm{P}$ NMR spectrum of $3 n$

NRSN II 387

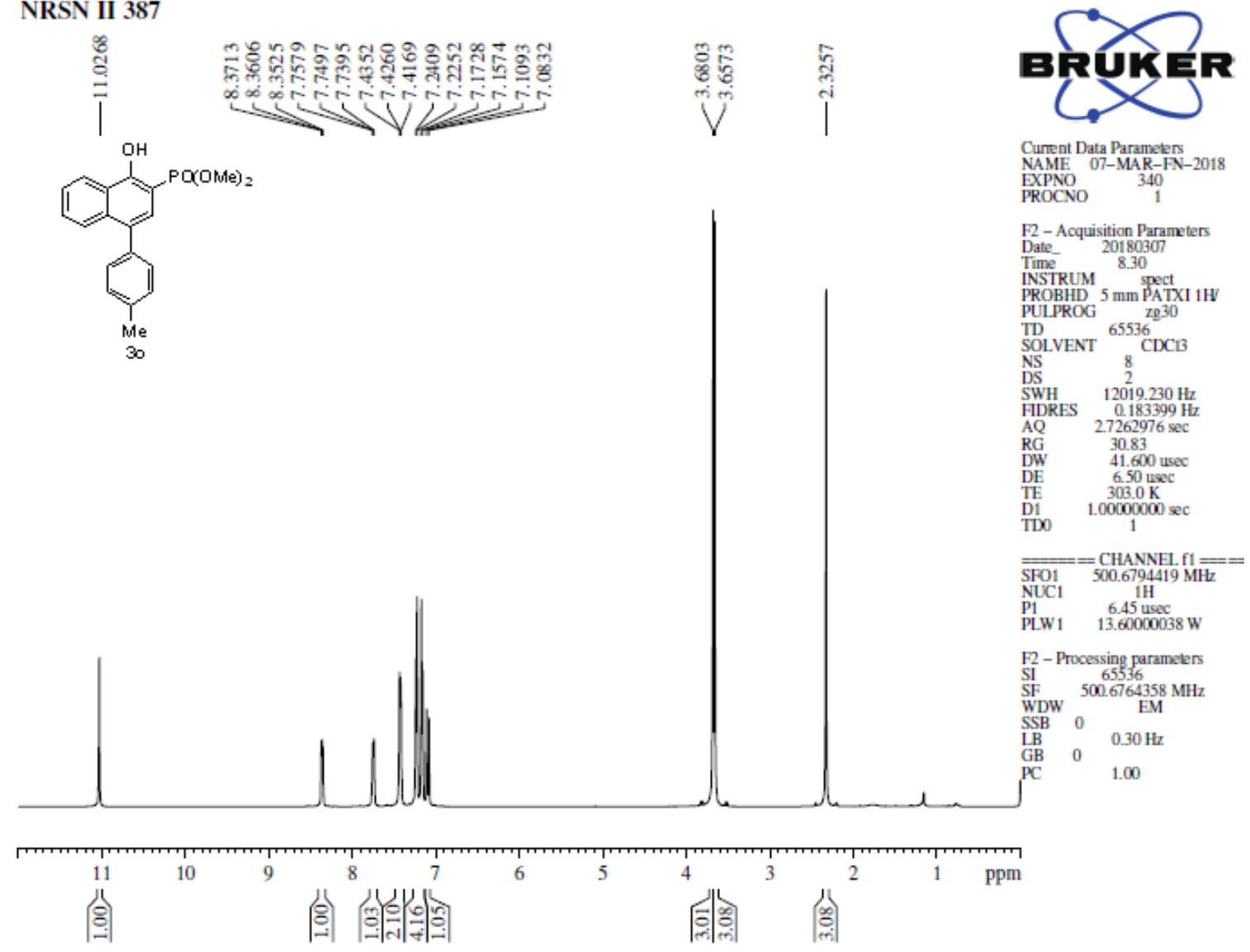

Spectrum 46: ${ }^{1} \mathrm{H}$ NMR spectrum of 30 


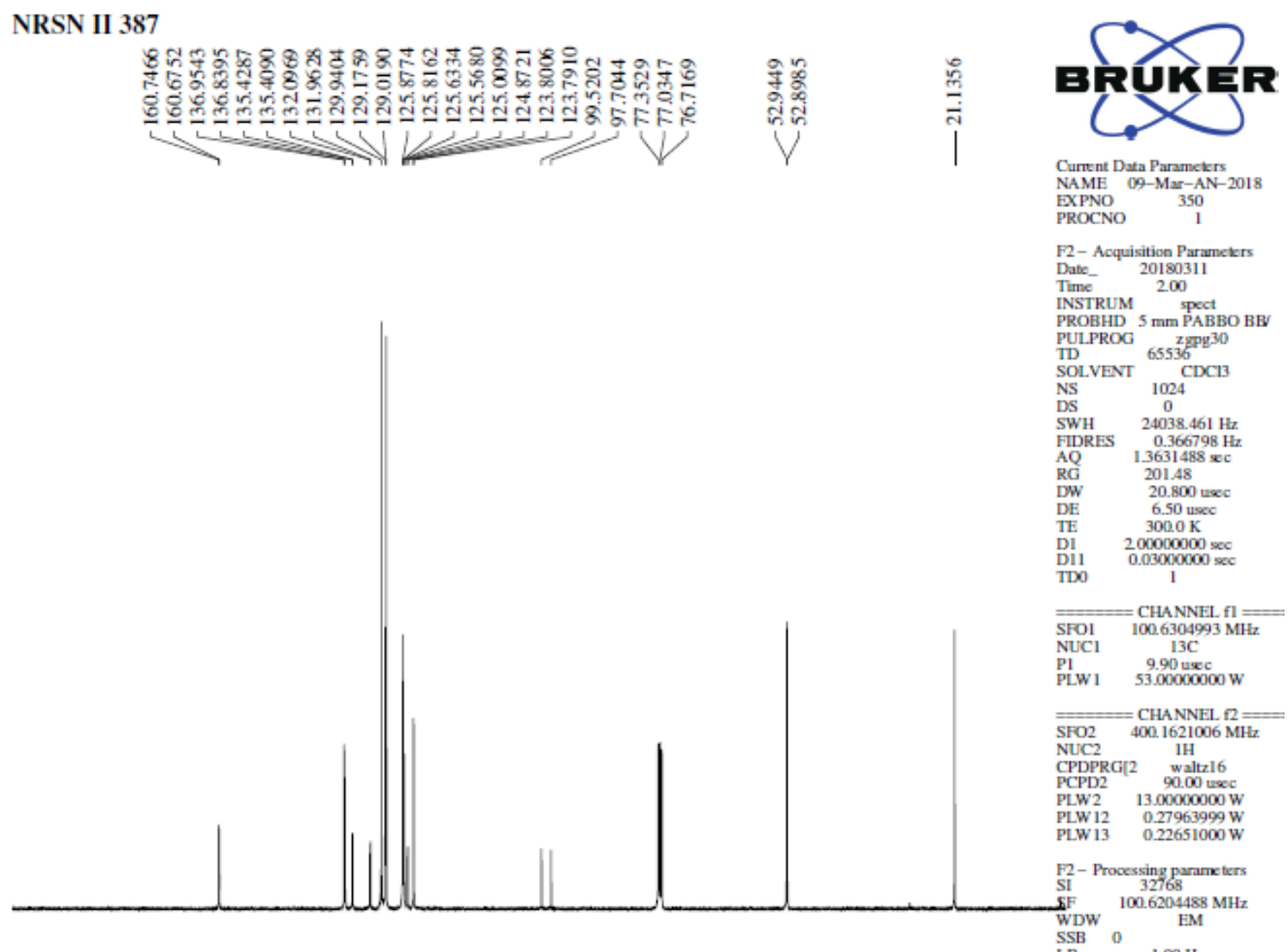

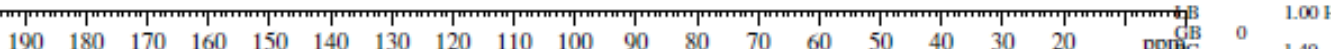

Spectrum 47: ${ }^{13} \mathrm{C}$ NMR spectrum of 30

NRSN-II-387
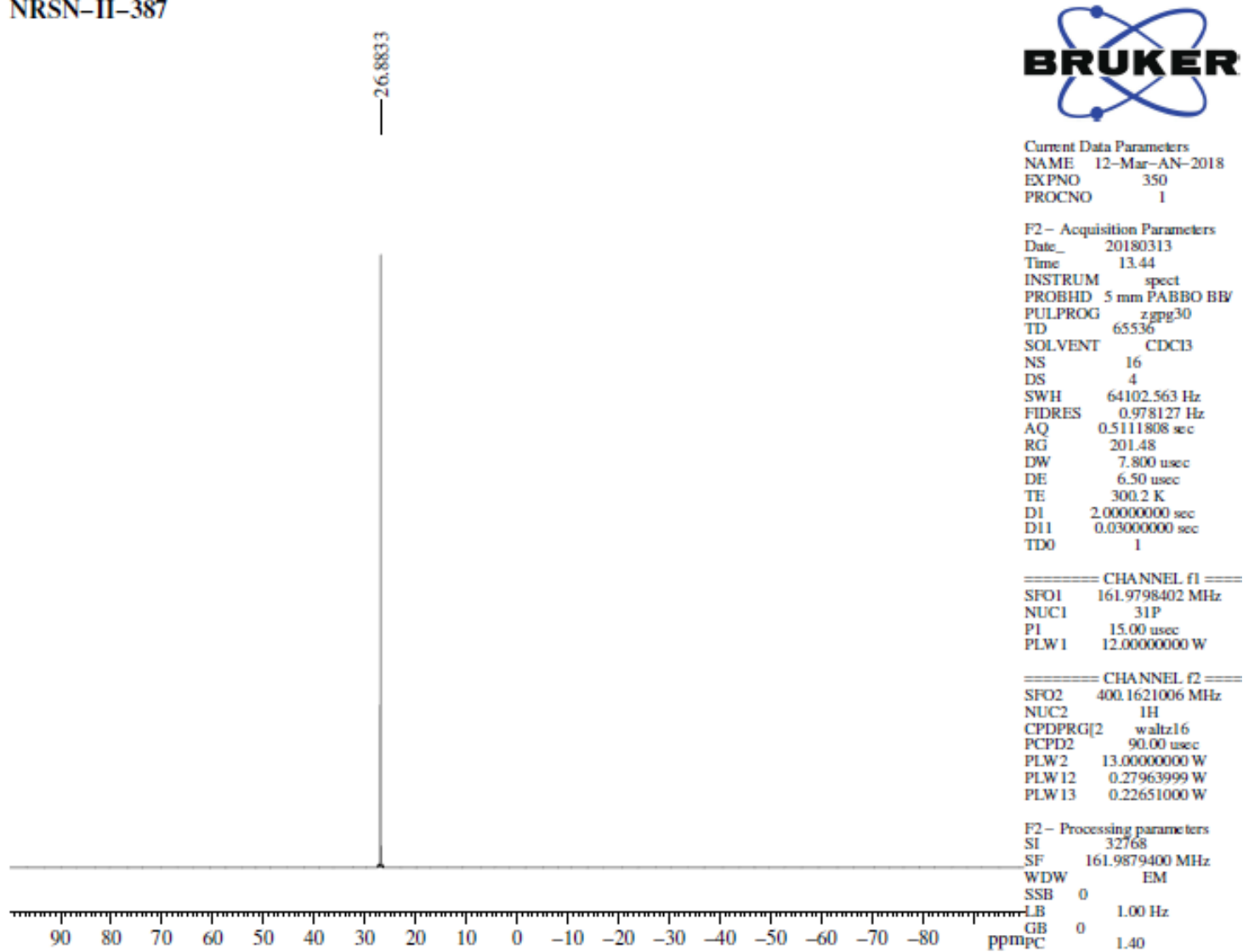

Spectrum 48: ${ }^{31} \mathrm{P}$ NMR spectrum of 30 


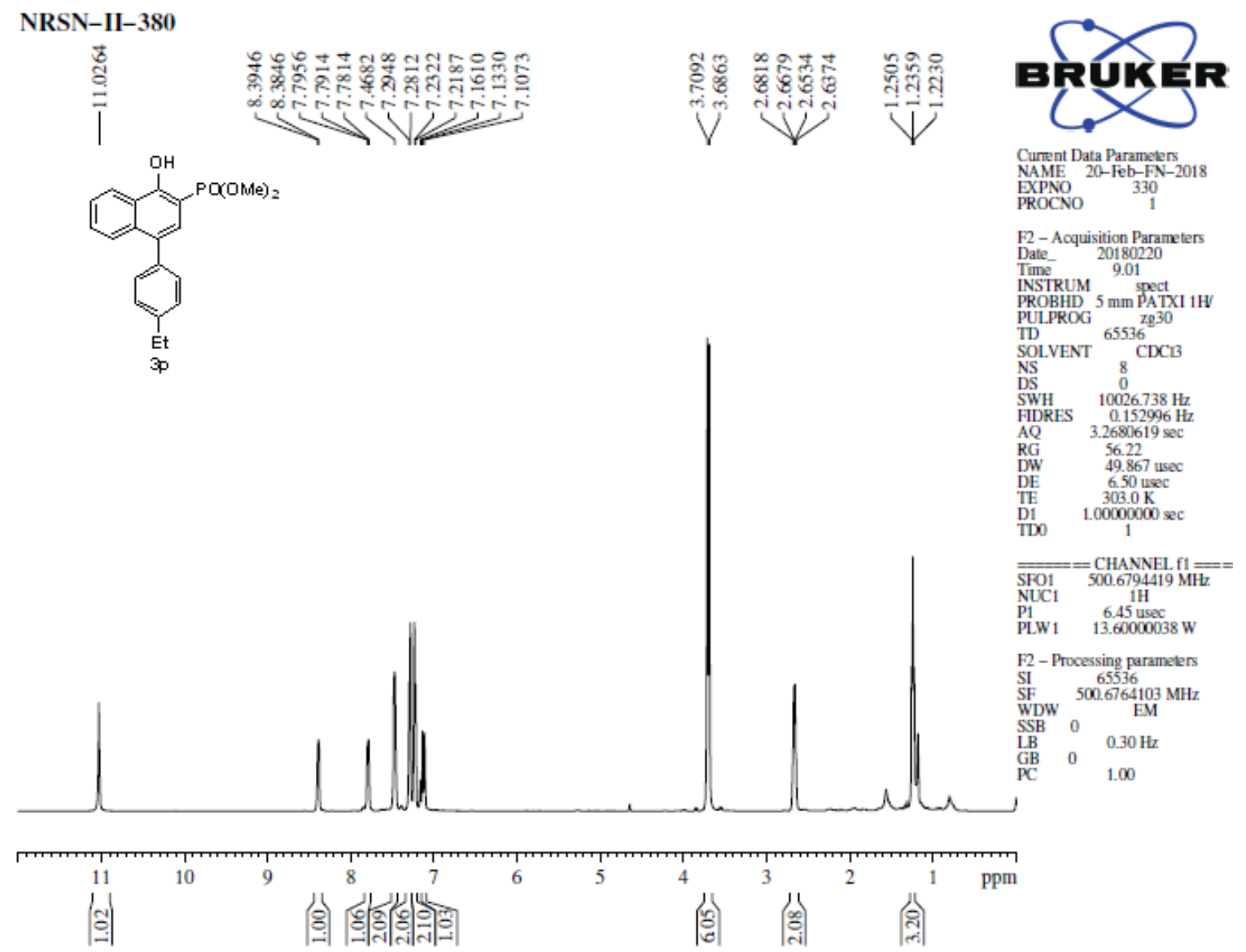

NRSN-II-380

Spectrum 49: ${ }^{1} \mathrm{H}$ NMR spectrum of $\mathbf{3 p}$
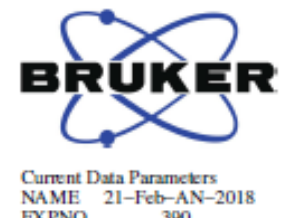

EXPNO $\quad 390$

PROCNO

390
1

F2- Acquisition Paramekrs

$\begin{array}{lc}\text { Date- } & 20180222 \\ \text { Time } & 19.42\end{array}$

INSTRUM spect

PULPROG 2 mpg 30

TD 65536 .

\begin{tabular}{ll} 
SOL.VENT & \multicolumn{1}{c}{ CDC } \\
NS & 40 \\
DS & 0 \\
SWH & 24038.461
\end{tabular}

SWH $24038.461 \mathrm{~Hz}$

AQ $\quad \begin{array}{ll}0.366798 \mathrm{~Hz} \\ \text { Fon }\end{array}$

RG 201.48

$\begin{array}{ll}\text { DW } & 20.800 \text { usec } \\ \text { DE } & 6.50 \text { usec }\end{array}$

$6.50 \mathrm{use}$
$300.0 \mathrm{~K}$

$\begin{array}{ll}\text { pl } & 20000000000 \\ \text { pil } & 0.03000000\end{array}$

TDO

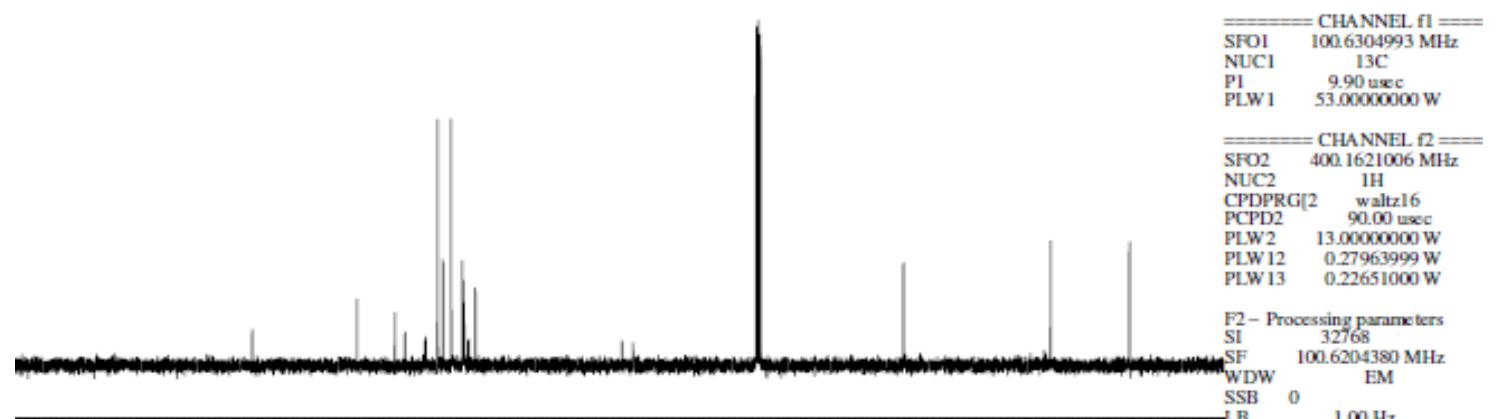

\begin{tabular}{llllllllllllllllllll|}
190 & 180 & 170 & 160 & 150 & 140 & 130 & 120 & 110 & 100 & 90 & 80 & 70 & 60 & 50 & 40 & 30 & 20 & PpmPC & 0 \\
\hline
\end{tabular}

Spectrum 50: ${ }^{13} \mathrm{C}$ NMR spectrum of 3p 


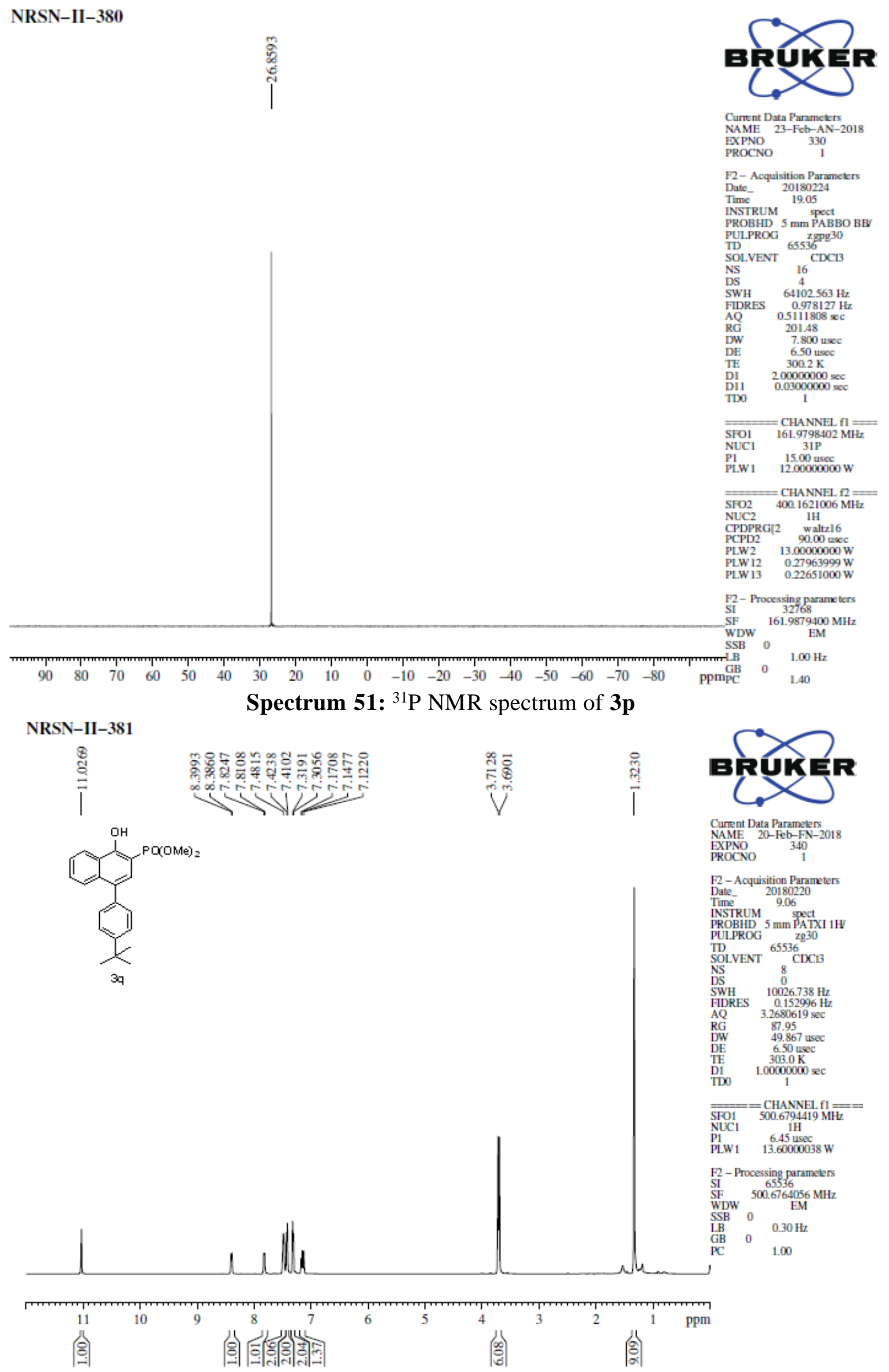

Spectrum 52: ${ }^{1} \mathrm{H}$ NMR spectrum of $\mathbf{3 q}$ 

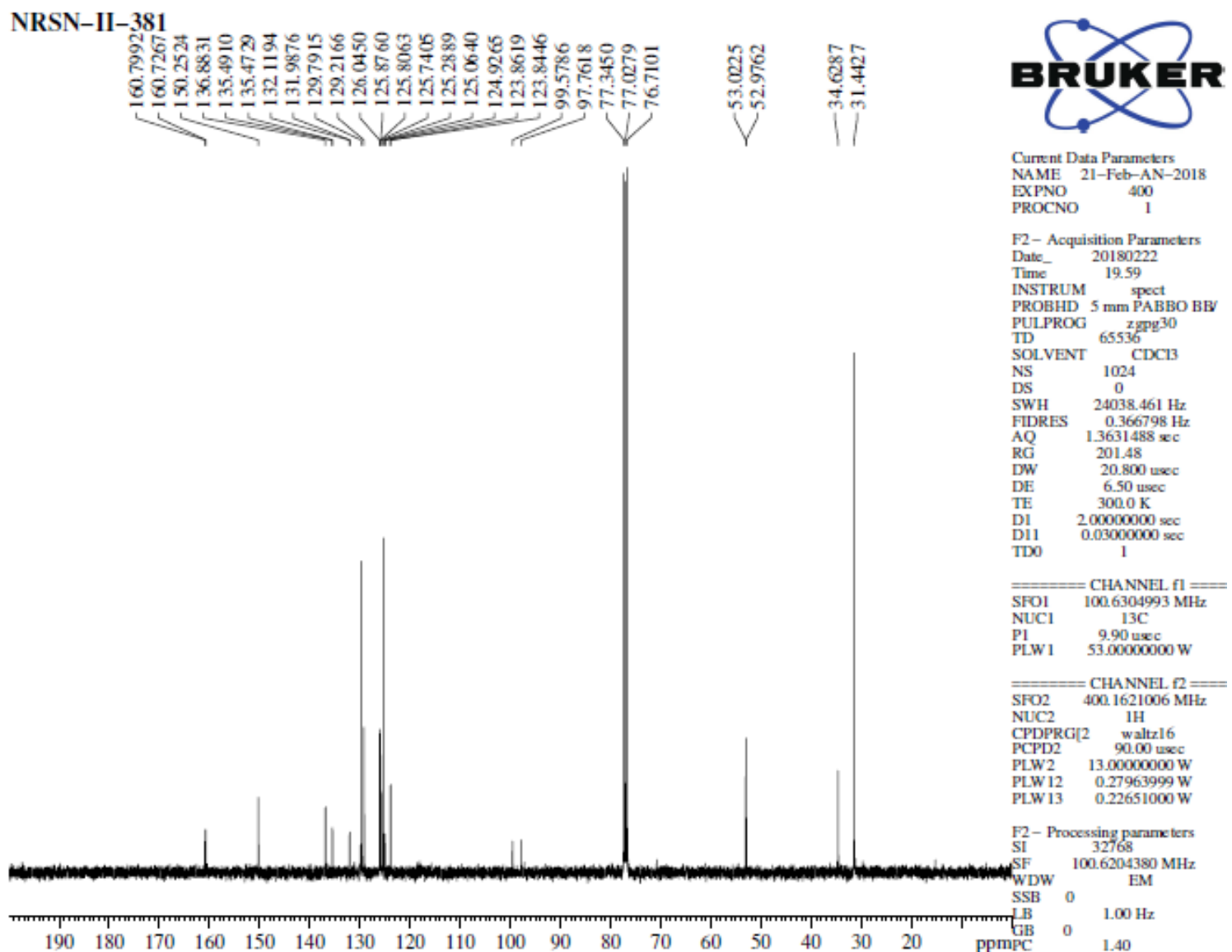

Spectrum 53: ${ }^{13} \mathrm{C}$ NMR spectrum of $\mathbf{3 q}$

NRSN-II-381

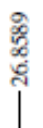

BRUKER

Current Data Parameters

EXPNO 340

2- Acquisition Paramekrs

Date_ 20180224

Time 19.10

INSTRUM spect

PROBHD 5 mm PABBO B

ULPROG $75 \mathrm{gmg}^{2} 30$

SOLVENT CDCI

NS 16

$\begin{array}{lll}\mathrm{DS} & 4 \\ \mathrm{SWH} & 64102.563 \mathrm{~Hz}\end{array}$

IDRES $\quad 0.978127 \mathrm{~Hz}$

AQ 0.5111808

DW $\quad 7.800$ usec

6.50 usec

$300.2 \mathrm{~K}$

$200000000 \mathrm{se}$

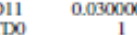

CHANNEL $\mathrm{fl}=$

SFO1 161.9798402 MHz

NUCl $31 \mathrm{P}$

P1 15.00 usec

SFO2 CHANNEL $\mathrm{f2}=$

NUC2 $1 \mathrm{H}$

CPDPRG [2 waltz16

PCPD2 90.00 usec

PLW $12 \quad 0.27963999 \mathrm{~W}$

PLW $130.22651000 \mathrm{~W}$

F1- Processing parame ters

SF $\quad 161.9879400 \mathrm{MHz}$

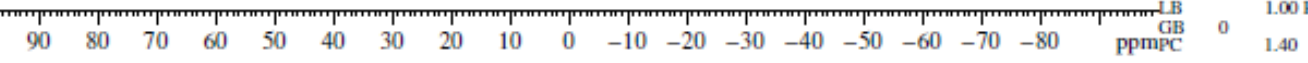

Spectrum 54: ${ }^{31} \mathrm{P}$ NMR spectrum of $\mathbf{3 q}$ 


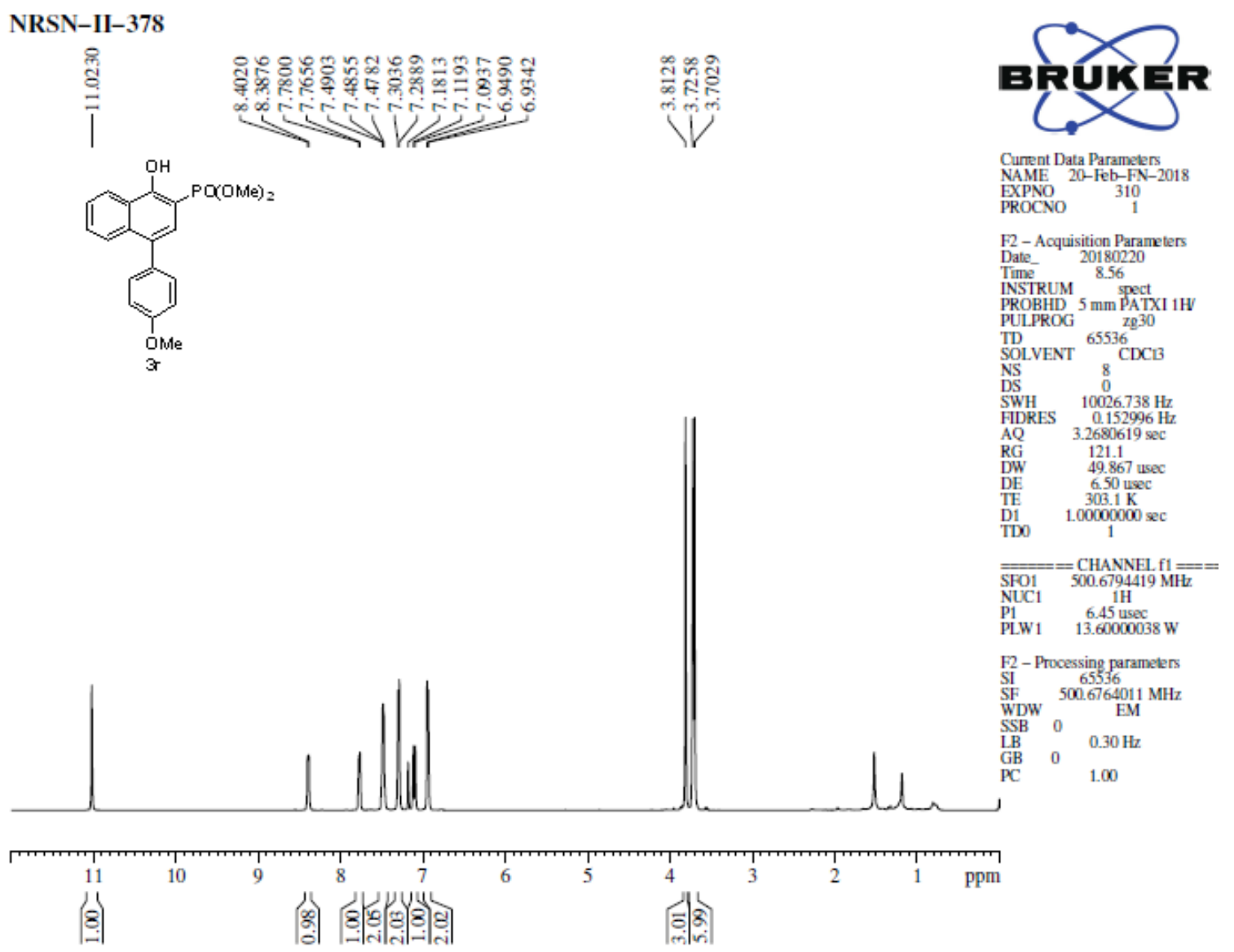

Spectrum 55: ${ }^{1} \mathrm{H}$ NMR spectrum of $3 \mathbf{r}$

NRSN-II-378

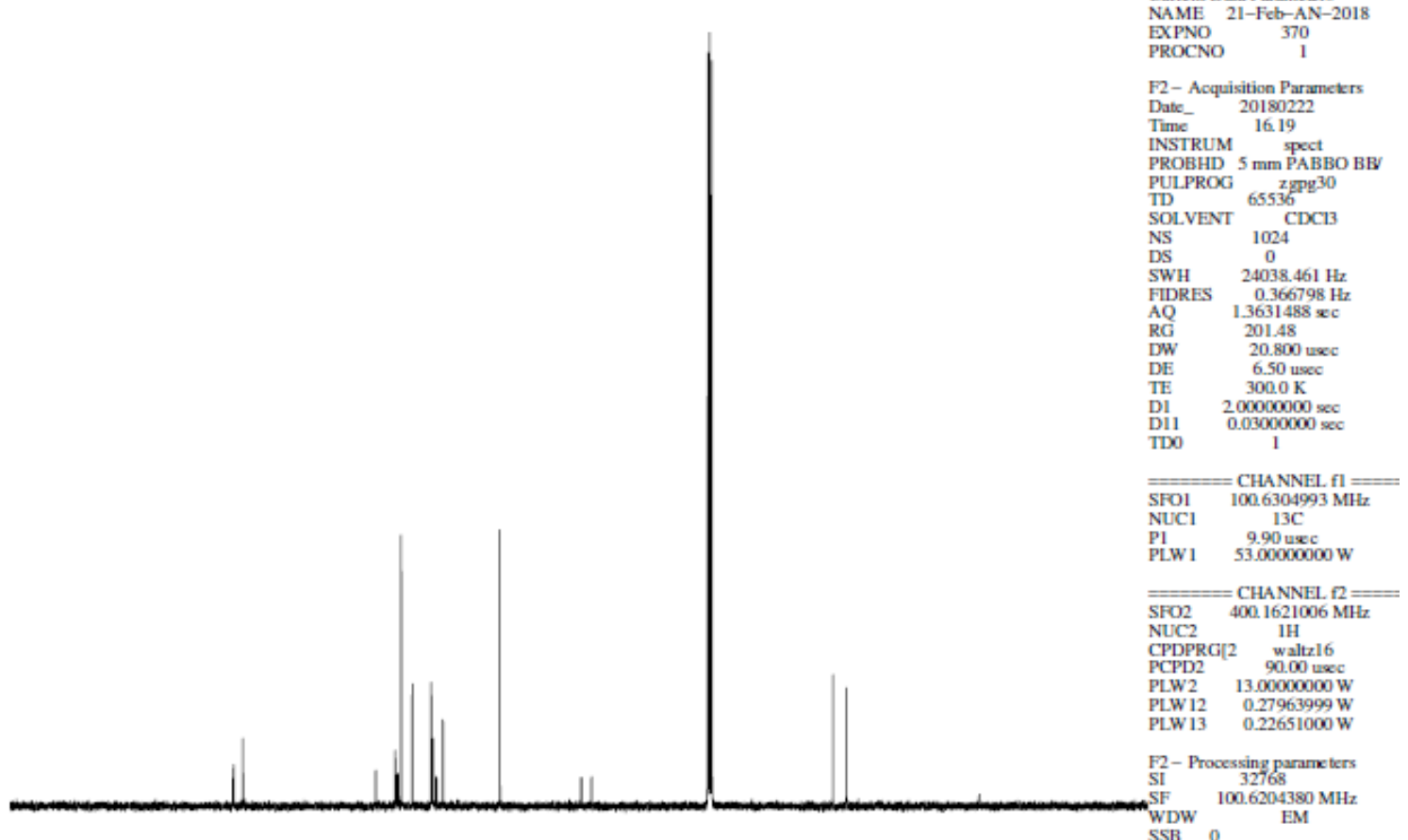

(2010

Current Data Parameters NAME 21-Feb-ANPROCNO

F2- Acquisition Parameters

Time- 16.19

NSTRUM spect

PULPROG zape 3 .

SOLVENT CDCI

NS $\quad 1024$

SWH $\quad 00$

FIDRES $0.366798 \mathrm{H}$

AQ $\quad 1.3631488$

RG 201.48

6.50 uvese

CHANNEL $\mathrm{fl}=$

9.90 usec
$530000000 \mathrm{~W}$

$\mathrm{SFO} 2$ CHANNEL $\mathrm{F}=$

waltz16

$13.00000000 \mathrm{~W}$

W13 $0.22651000 \mathrm{~W}$

Spectrum 56: ${ }^{13} \mathrm{C}$ NMR spectrum of $\mathbf{3 r}$ 


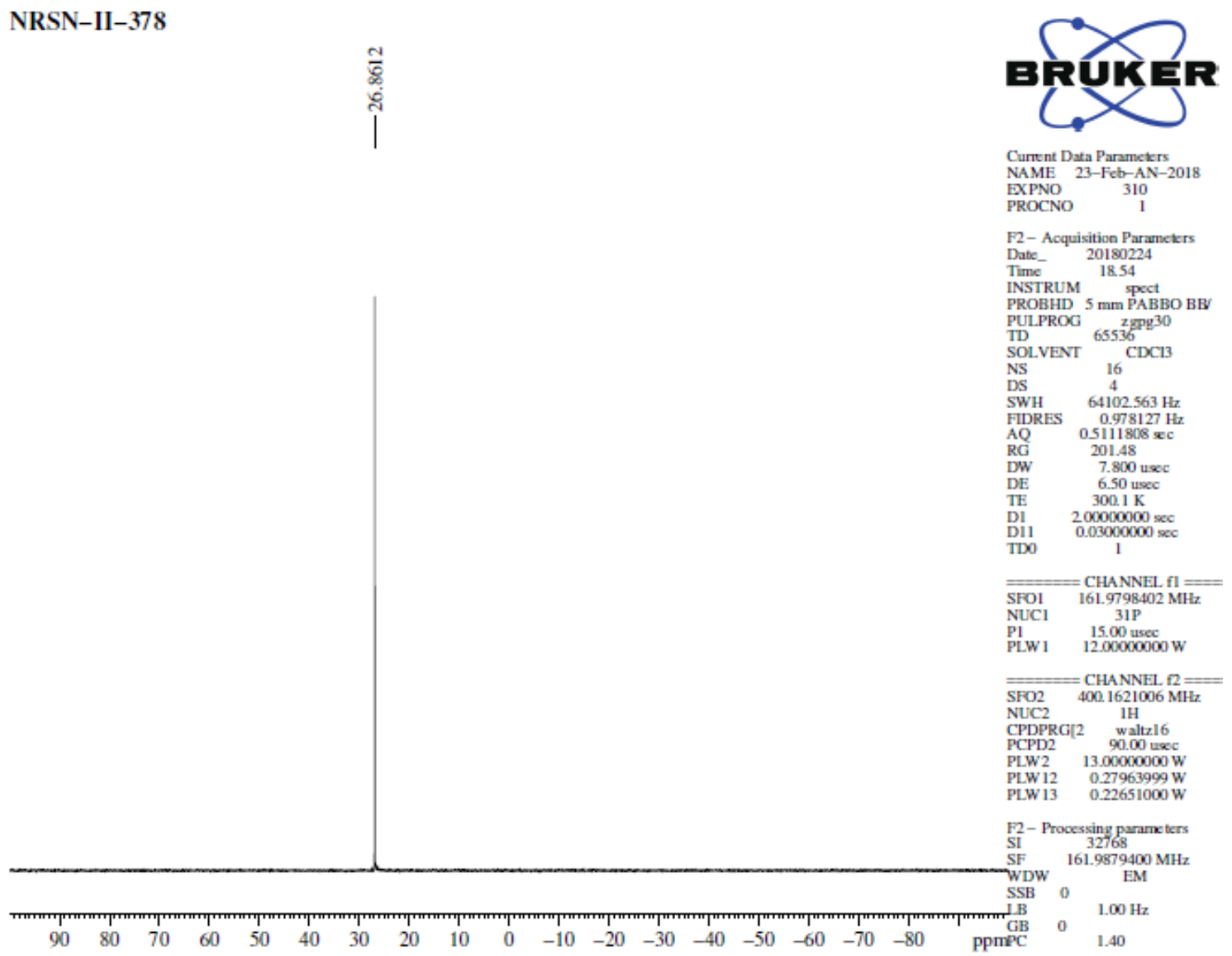

Spectrum 57: ${ }^{31} \mathrm{P}$ NMR spectrum of $\mathbf{3 r}$

NRSN II 385

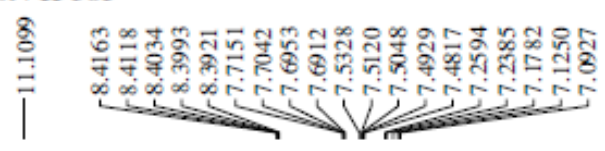

(come)
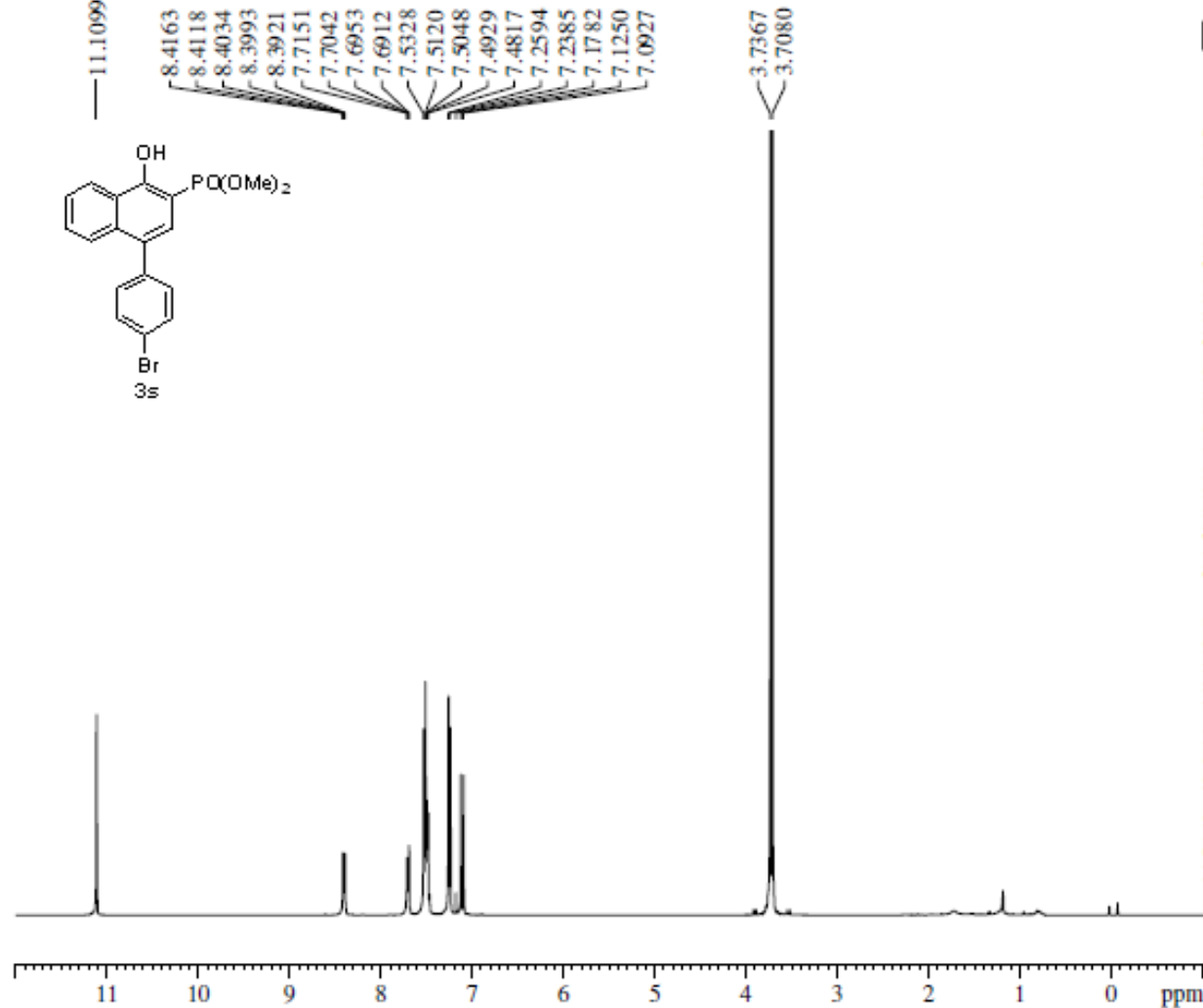

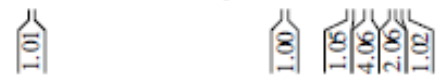

常

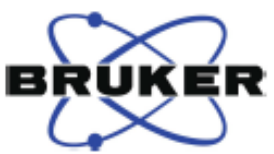

Cuntent Data Parameters NAME 27-Feb-FN-2018 PRPNO

F2 - Acquisition Parameters

Date_ 20180227

PROBHD $5 \mathrm{~mm}$ PABBO BB/

${ }_{\text {PULPROG }}{ }_{\mathrm{Zg} 30}$

$\begin{array}{lr}\text { PUL.PROG } & \text { 2g } 30 \\ \text { TD } & 65536\end{array}$

SOLVENT $\quad$ CDS36

$\begin{array}{ll}\text { NS } & 8 \\ \text { DS } & 0 \\ \text { SWH } & 9615.385\end{array}$

SWH $\quad 9615.385 \mathrm{~Hz}$

$\begin{array}{ll}\text { FDRES } & 0.146719 \mathrm{H} \\ \text { AQ } & 3.4078720 \mathrm{sec}\end{array}$

$\begin{array}{lr}\mathrm{AQ} & 3.40787 \\ \mathrm{RG} & 31.99 \\ \mathrm{DW} & 52.000\end{array}$

31.99
52000 usec

6.50 usec

$300.0 \mathrm{~K}$

D1 $1.00000000 \mathrm{sec}$

$\overline{\text { SFO1 }}=$ CHANNEL $\mathrm{f} 1=$

NUC1 400.16297

$\begin{array}{ll}\text { P1 } & 13.20 \text { usec } \\ \text { PLW1 } & 13.00000000 \mathrm{~W}\end{array}$

F2 - Processing paramelers

$\begin{array}{ll}\text { SI } & 65536 \\ \text { SF } & 400.1605420 \mathrm{MHz}\end{array}$

WDW $400.1605420 \mathrm{M}$

$\underset{\mathrm{LB}}{\mathrm{SB}} \quad 0.30 \mathrm{~Hz}$

$\mathrm{GB}$

PC $\quad 1.00$

Spectrum 58: ${ }^{1} \mathrm{H}$ NMR spectrum of $3 \mathrm{~s}$ 


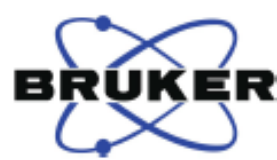
Curnent Data Parameders
NAME 06-Mar-FN-2018 EXPNO

B2- Acquisition Parmeters

Date_ 20180306

INSTRUM 8.46

PROBHD 5 mm PABBO BB

PULPROG z: zpg 30

TD 65536

SOLVEN $\mathrm{CDCl}^{2}$

NS

$\begin{array}{ll}\text { DS } & 4 \\ \text { SWH } & 24038.461 \mathrm{H}\end{array}$

FIDRES 0366798 H

AQ $1.3631488 \times 2$

RG 201.48

$\begin{array}{ll}\text { DW } & 20.800 \text { usec } \\ \text { DE } & 6.50 \text { useec }\end{array}$

$3000 \mathrm{~K}$

DI $2000000000 \mathrm{sec}$

TDO 1

CHANNEL $\mathrm{fl}=$

NUCl $102.6304993 \mathrm{M}$

Pl
PLW1 $93.900000000 \mathrm{~W}$

$=$ CHANNEL $\mathrm{R}=$

SFO2 $400.1621006 \mathrm{MH}$

NUC2 $21 \mathrm{H}$

CPDPRG[2 waltzl6
PCPD2
90.00 usec

PLW2 13,00000000 W

PLW12 $0.27963999 \mathrm{~W}$

F2- Processing parame ters

SF $\quad 100.6204380 \mathrm{MHz}$

WDW EM

$\begin{array}{lll}\mathrm{SSB} & 0 & \\ \mathrm{~B} & 1.00 \mathrm{~Hz}\end{array}$

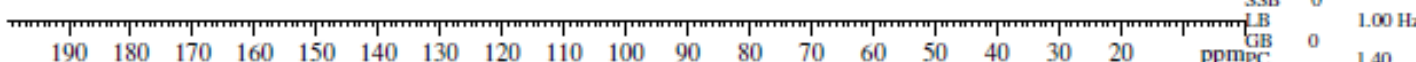

Spectrum 59: ${ }^{13} \mathrm{C}$ NMR spectrum of $3 \mathrm{~s}$

NRSN II 385

ปั.

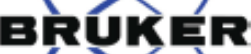

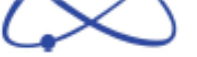

Curnent Data Parameters

NAME O9-Mar-AN-2018 PROCNO

F2- Acquisition Parameters

Date_ 2018031

Tims

207

PROBHD 5 mm PABBO BB

PULPROG $2 \mathrm{gPg} 30$

SOLVENT $\quad$ CDCCI

NS 32

$\begin{array}{lcl}\text { DS } & 32 \\ \text { SWH } & 4 & 4402563 \mathrm{H}\end{array}$

IDRES $\quad 0.978127 \mathrm{H}$

AQ $0.5111808 \times$

RG 201.48

$\begin{array}{ll}\mathrm{DW} & 7.800 \text { use } \\ \mathrm{DE} & 6.50 \text { usec }\end{array}$

$\begin{array}{ll}\text { pI } & 200000000 \mathrm{sec} \\ \text { Dil } & 0.03000000 \mathrm{sec}\end{array}$

TDO

FOI CHANNEL $\mathrm{fl}=$

NUC1 31P

$\begin{array}{ll}\text { Pl } & 15.00 \text { usec } \\ \text { PLW1 } 12.00000000 \mathrm{~W}\end{array}$

$=$ CHANNEL $\mathrm{R}=$

FO2 400.1621006

CPDPRG 2 waltz16
PCPD2
90.00 uxe

PLW2 $13.00000000 \mathrm{~W}$

PLW13 $0.27651000 \mathrm{~W}$

2 - Processing parame ter

SI $\quad 32768$ MH

WDW EM

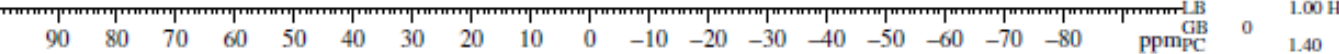

Spectrum 60: ${ }^{31} \mathrm{P}$ NMR spectrum of $3 \mathrm{~s}$ 


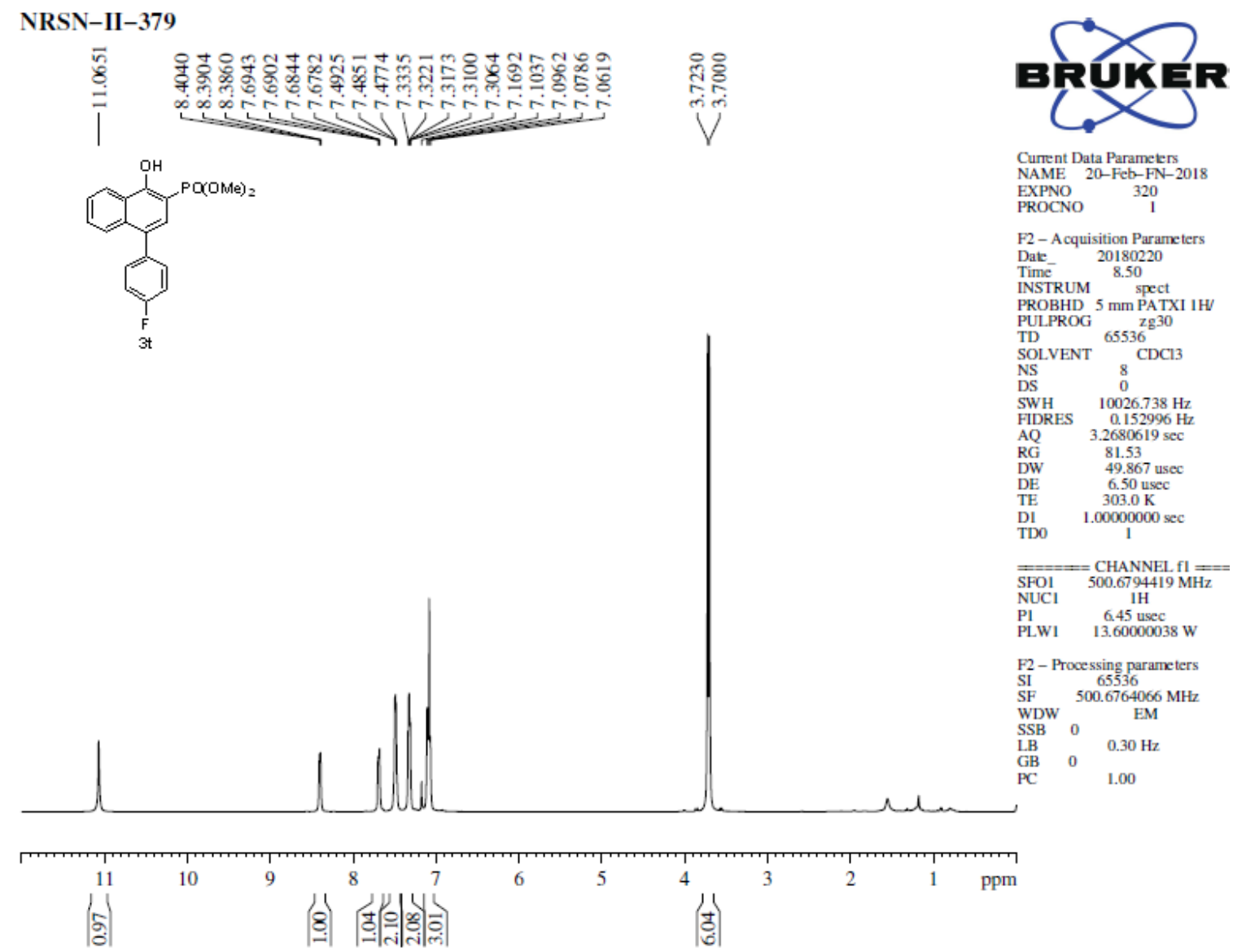

Spectrum 61: ${ }^{1} \mathrm{H}$ NMR spectrum of $\mathbf{3 t}$

NRSN-II-379
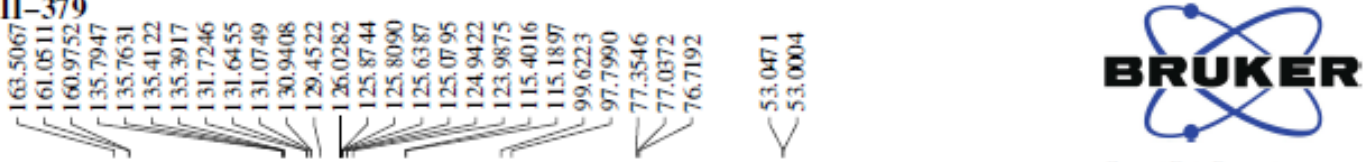

Current Data Parameters
NAME 21-Feb-AN-2018

$\begin{array}{lr}\text { EXPNO } & 380 \\ \text { PROCNO } & 1\end{array}$

F2 - Acquisition Paramelers

Date_ 20180222

Time 14.47

INSTRUM 1 pect
PROBHD $5 \mathrm{~mm}$ PABBO BB

PROBHD $5 \mathrm{~mm}$ PABBO

PULPROG 6553 zpg $^{30}$

SOLVENT ${ }^{65530} \mathrm{CDCI3}$

NS 512

$\begin{array}{lc}\text { DS } & 512 \\ \text { SWH } & 0 \\ 24038.461 \mathrm{~Hz}\end{array}$

$\begin{array}{ll}\text { SWH } & 24038.461 \mathrm{~Hz} \\ \text { FIDRES } & 0.366798 \mathrm{r}\end{array}$

AQ $1.3631488 \mathrm{x}$

DW 20.800 usec

DE $\quad 6.50$ us

$\begin{array}{ll}\text { DI } & 200000000 \mathrm{sec} \\ \text { DII } & 0.030000000 \mathrm{sec}\end{array}$

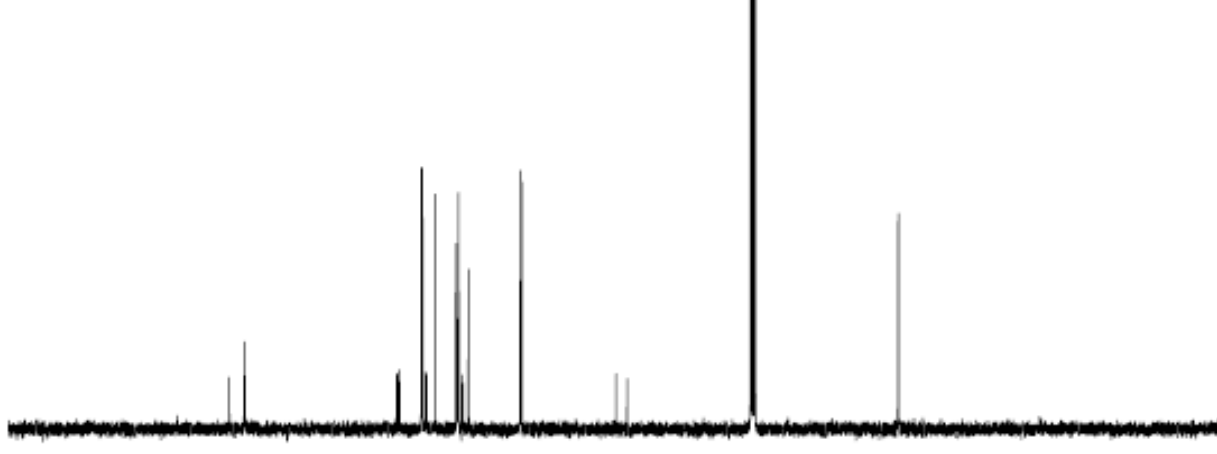

CHANNEL $\mathrm{fl}=$

SFO1 $1026304993 \mathrm{M}$

$\begin{array}{ll}\text { SFOI } & 10026304993 \\ \text { NUCl } & 13 \mathrm{C}\end{array}$

PLW1 53.00000000 W

CHANNEL $\mathrm{n} 2$

$4021621006 \mathrm{MI}$

$\mathrm{NUC2}_{\text {CPDPR } 2 ~}^{\text {1H }}$ waltz16

PCPD2 90.00 used

PLW2 $13.00000000 \mathrm{~W}$

$\begin{array}{ll}\text { PLW 12 } & 0.27963999 \mathrm{~W} \\ \text { PLW 13 } & 0.22651000 \mathrm{~W}\end{array}$

F2- Processing parameters

102768

WDW EM

\begin{tabular}{llllllllllllllllllll}
190 & 180 & 170 & 160 & 150 & 140 & 130 & 120 & 110 & 100 & 90 & 80 & 70 & 60 & 50 & 40 & 30 & 20 & ppmPC & 0 \\
\hline
\end{tabular}

Spectrum 62: ${ }^{13} \mathrm{C}$ NMR spectrum of $\mathbf{3 t}$ 


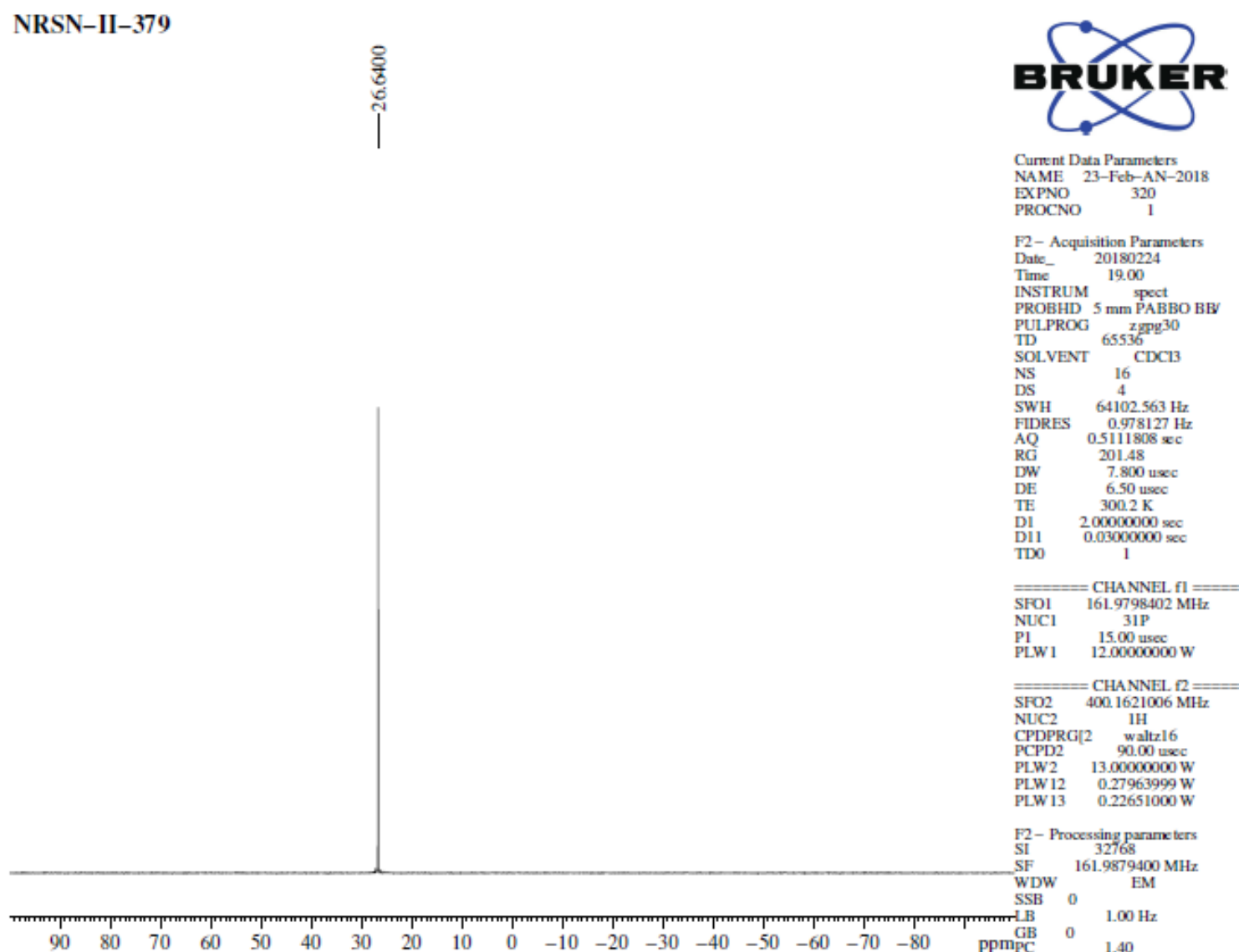

Spectrum 63: ${ }^{31} \mathrm{P}$ NMR spectrum of $\mathbf{3 t}$

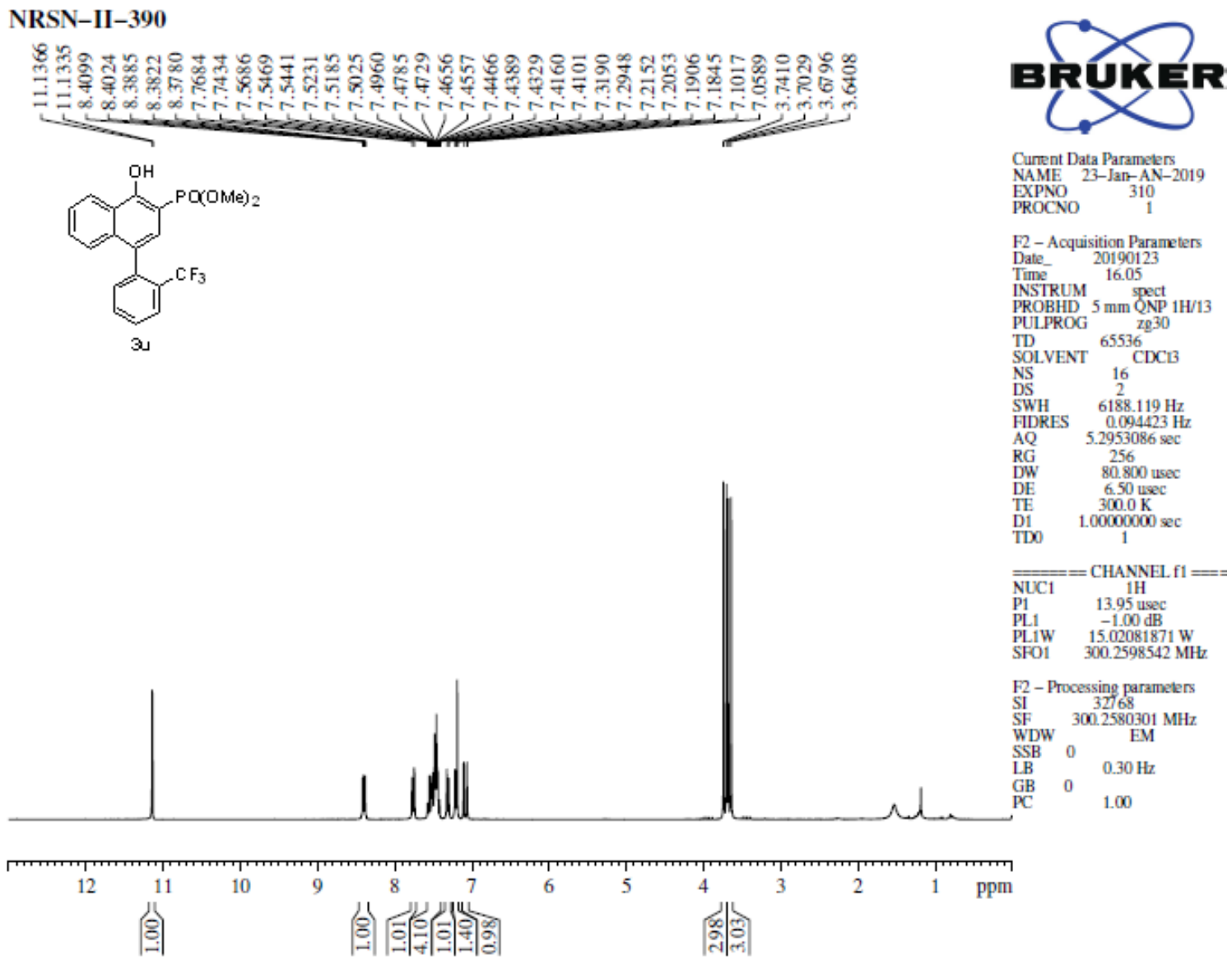

Spectrum 64: ${ }^{1} \mathrm{H}$ NMR spectrum of $3 u$ 


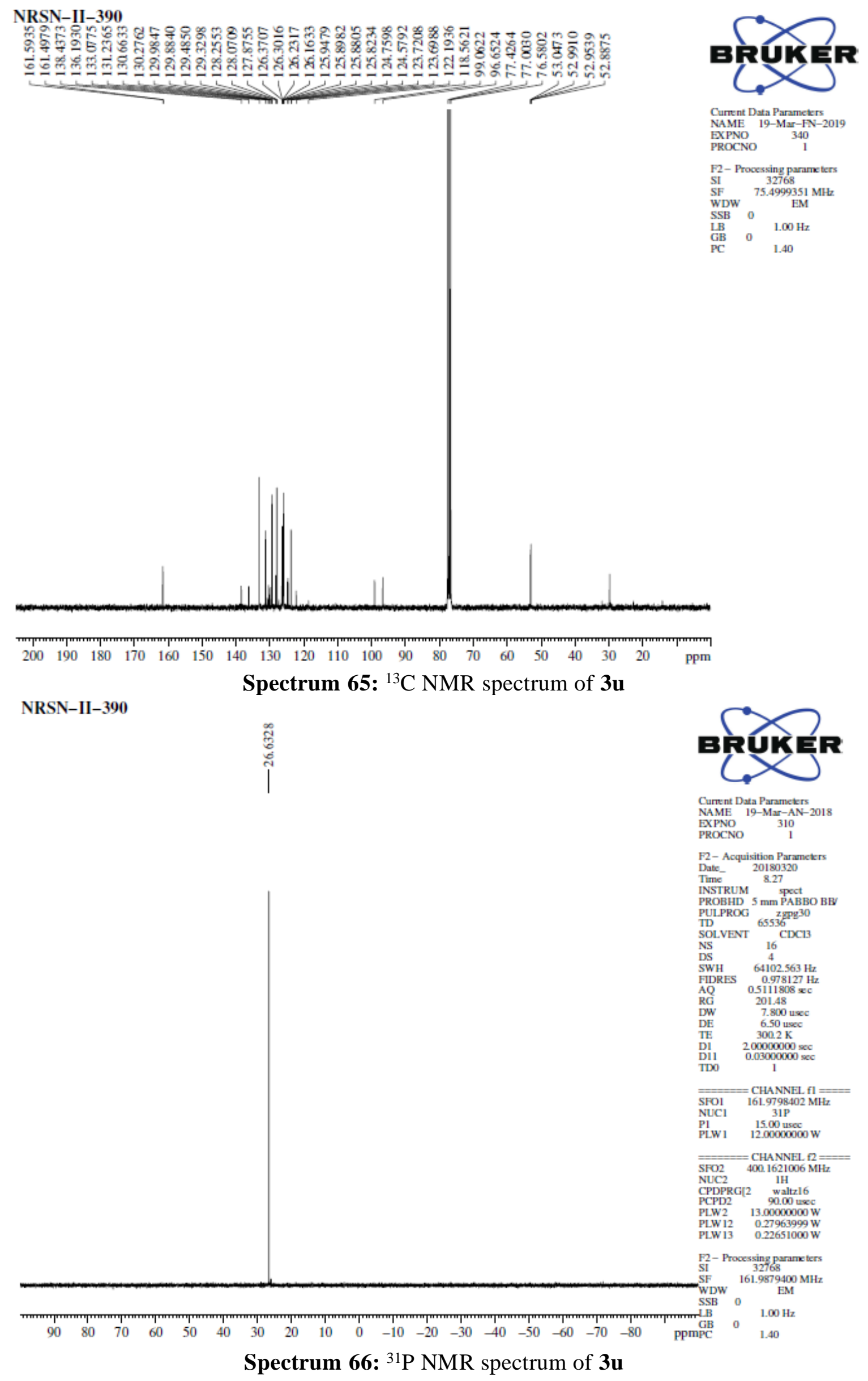




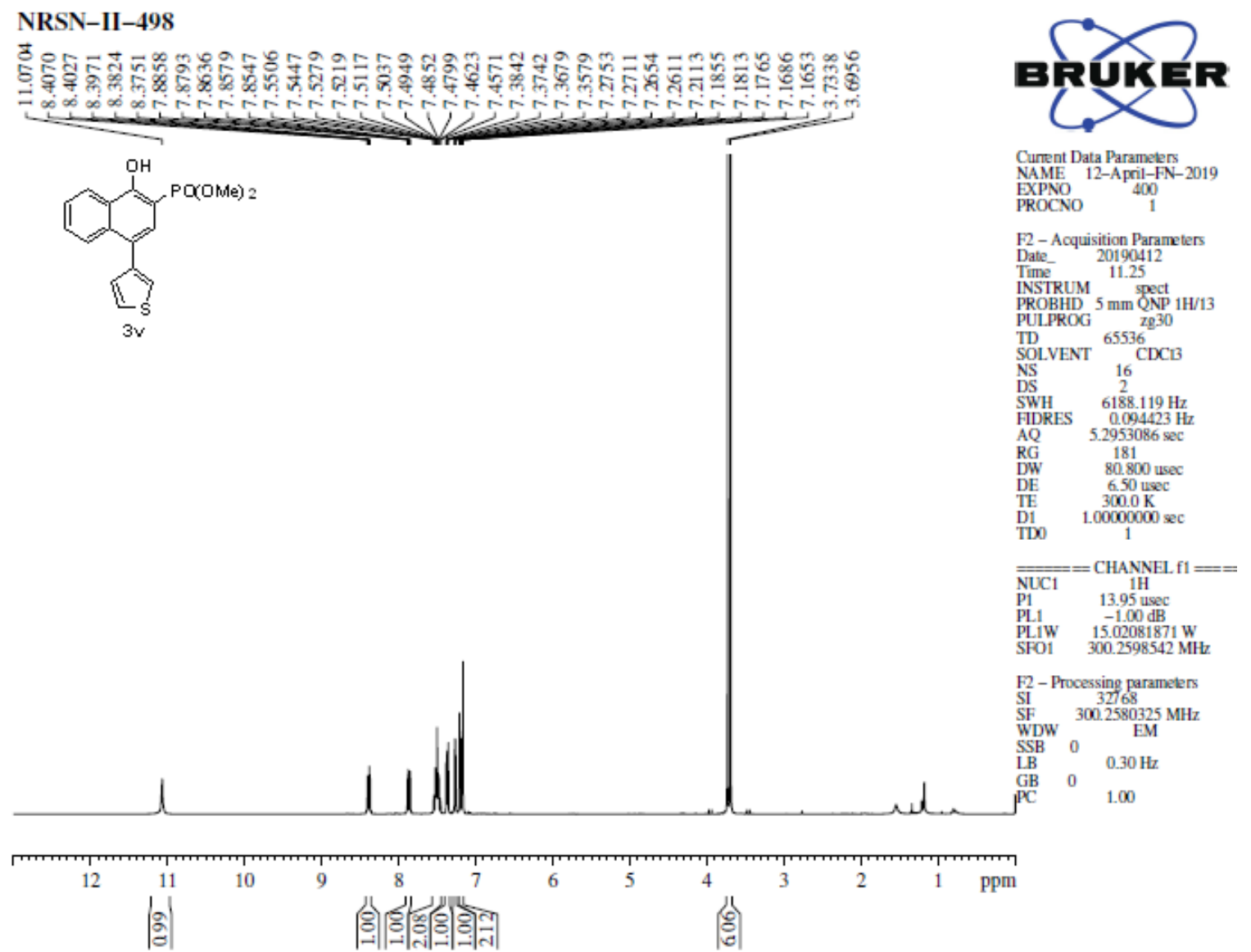

NRSN-II-498

Spectrum 67: ${ }^{1} \mathrm{H}$ NMR spectrum of $3 \mathrm{v}$

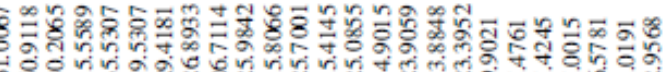

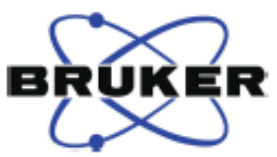

Current Data Parameters

NAME $15-\mathrm{Apr}-\mathrm{FN}-2019$

EXPNO

F2 - Processing parameters

$\begin{array}{ll}\text { SI } & 32768 \\ \text { SF } & 75.4999368 \mathrm{MH}\end{array}$

WDW EM

$\begin{array}{lll}\text { LSB } & 0 & 2.00 \mathrm{~Hz}\end{array}$

$\begin{array}{lll}\mathrm{GB} & 0 & 1.40\end{array}$

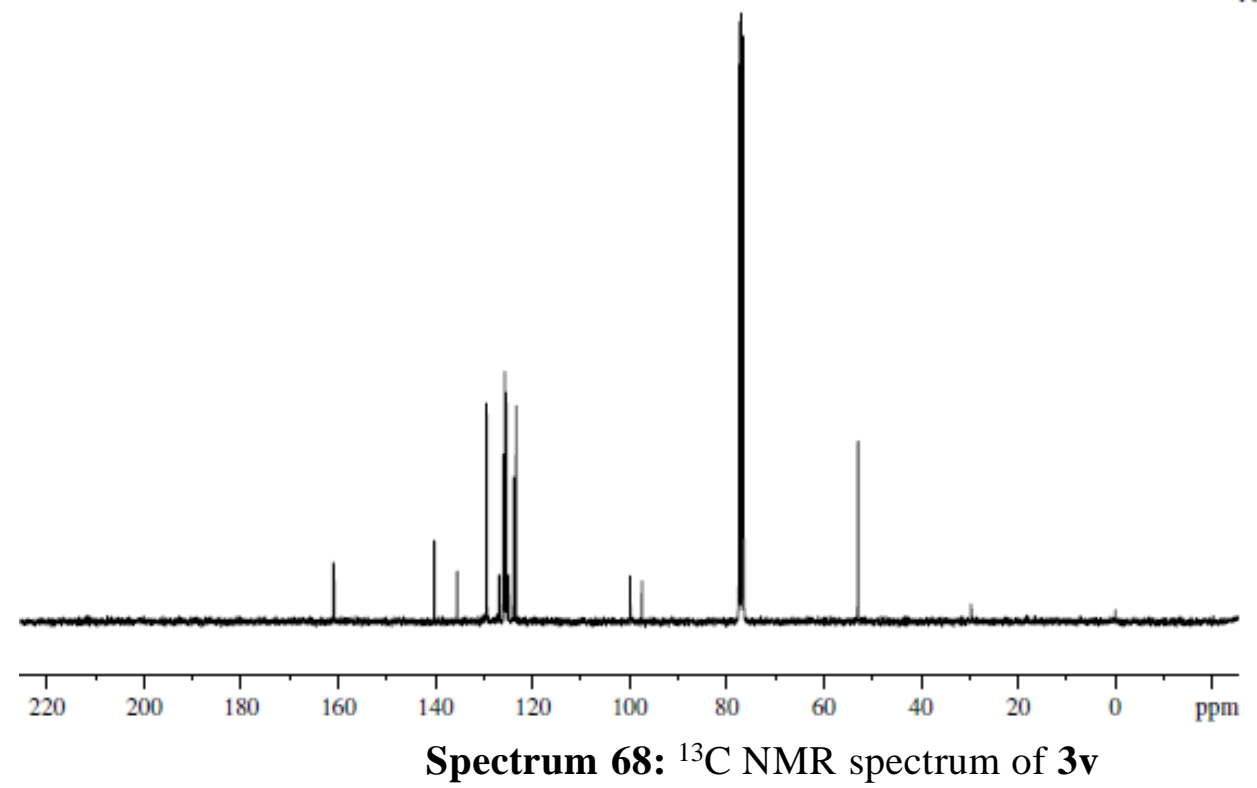




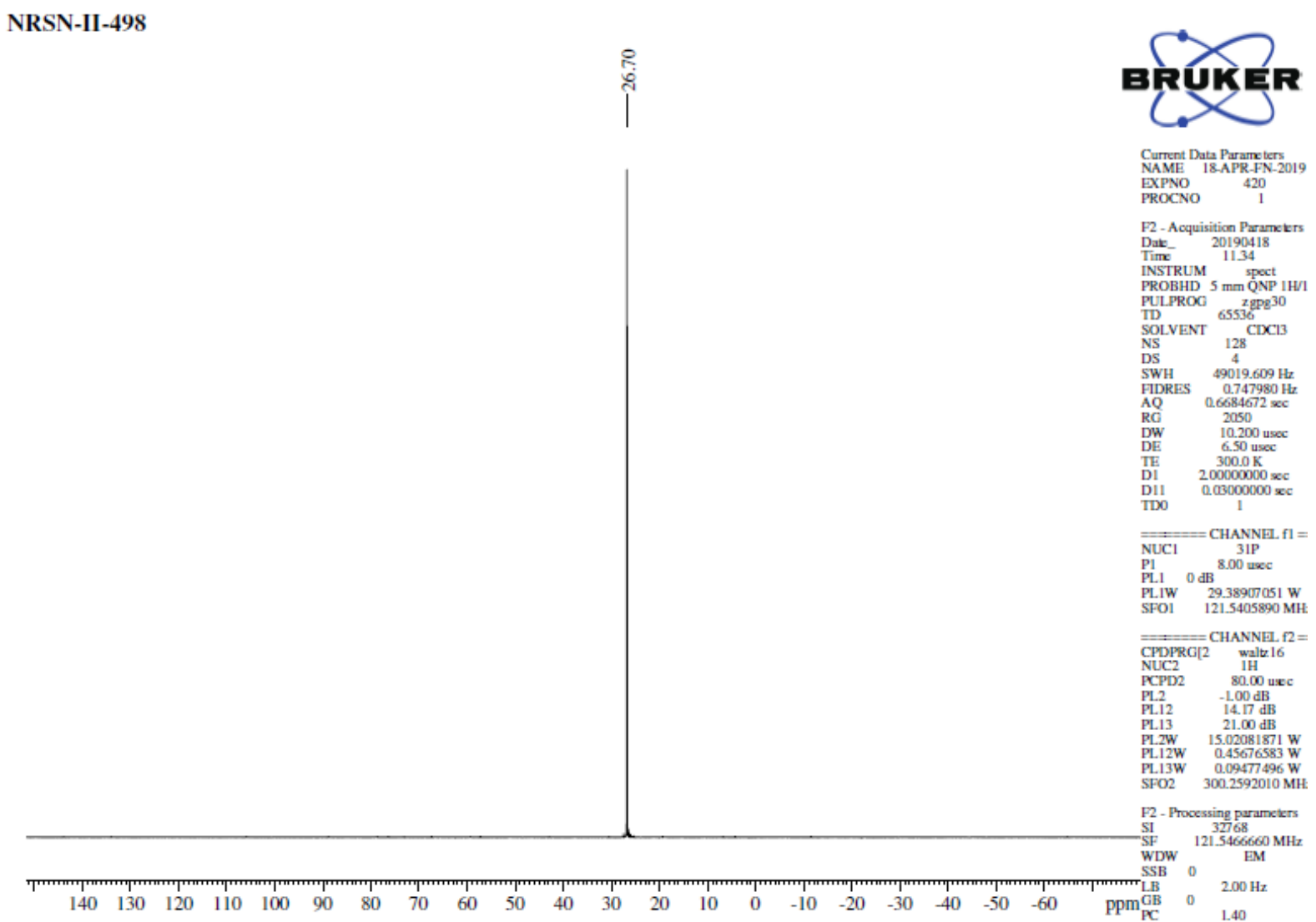

Spectrum 69: ${ }^{31} \mathrm{P}$ NMR spectrum of $\mathbf{3 v}$

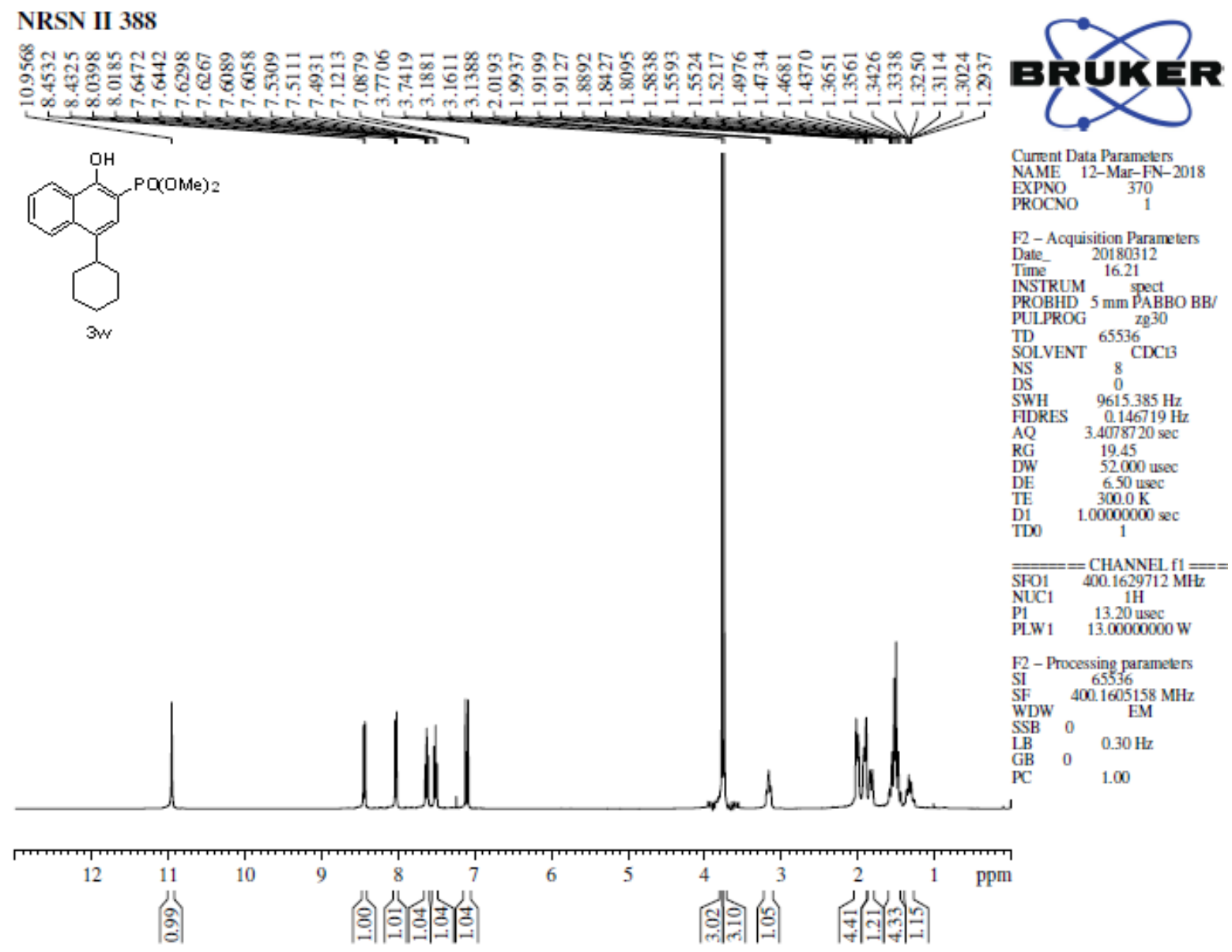

Spectrum 70: ${ }^{1} \mathrm{H}$ NMR spectrum of $\mathbf{3 w}$ 

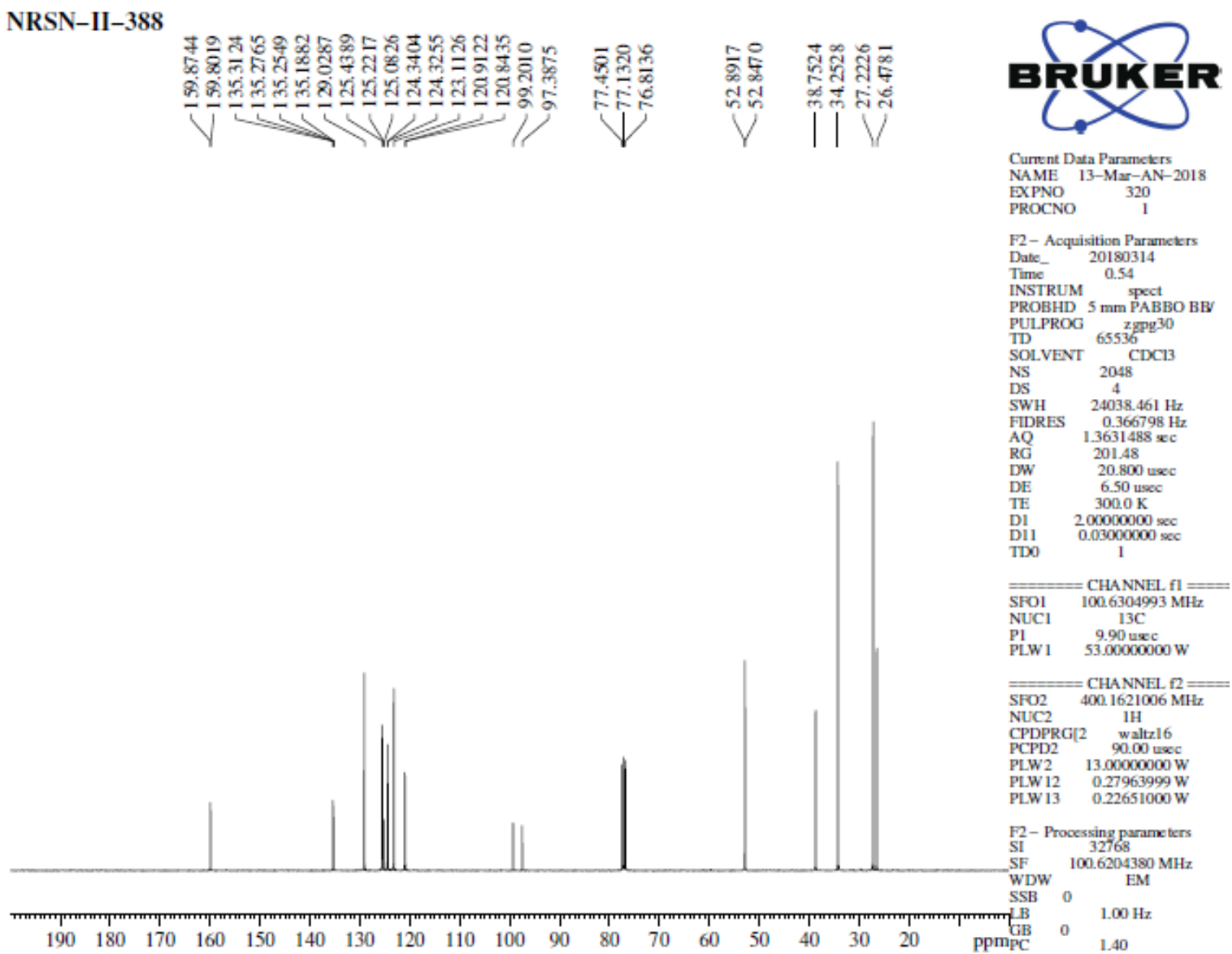

Spectrum 71: ${ }^{13} \mathrm{C}$ NMR spectrum of $\mathbf{3 w}$

NRSN-II-388

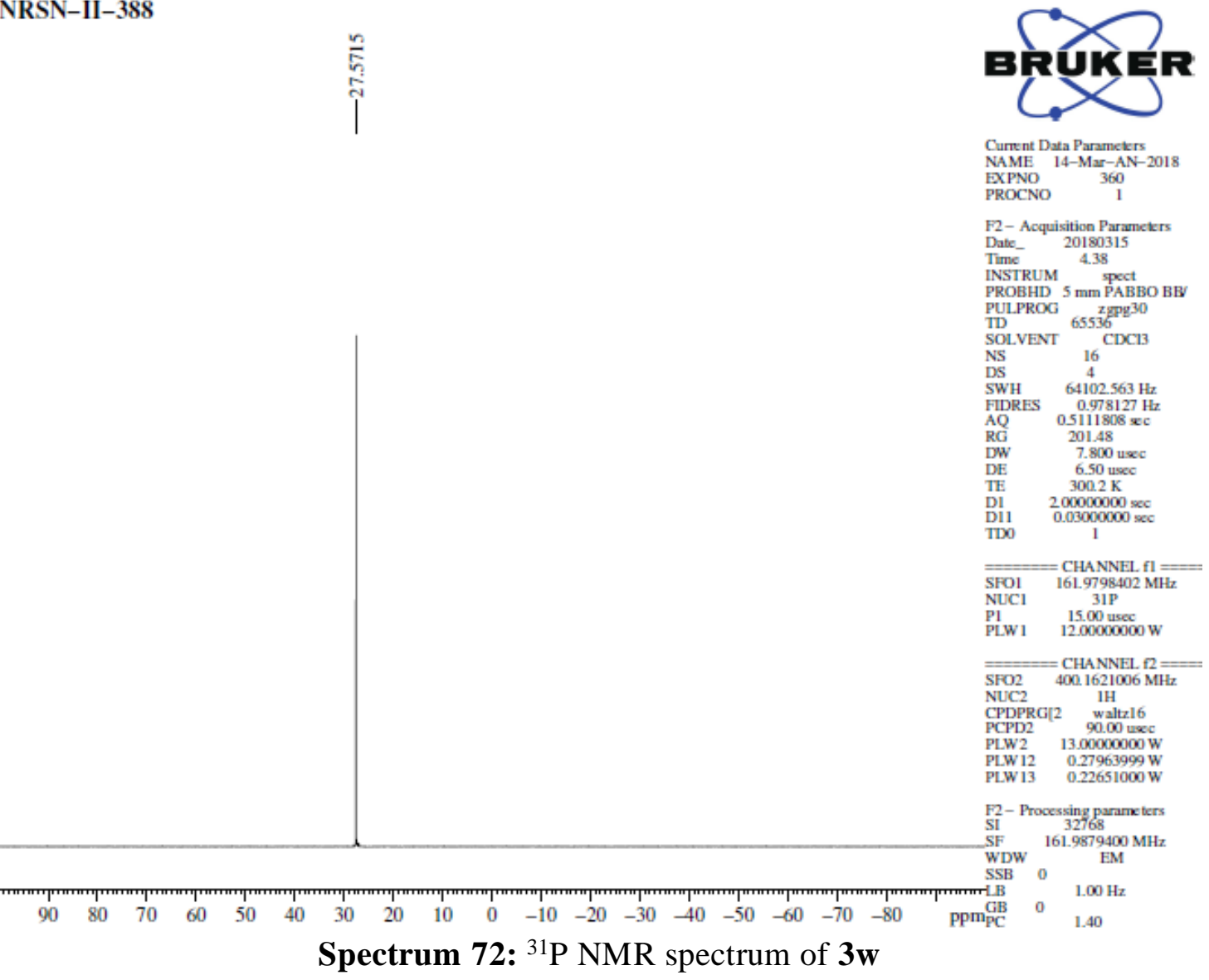




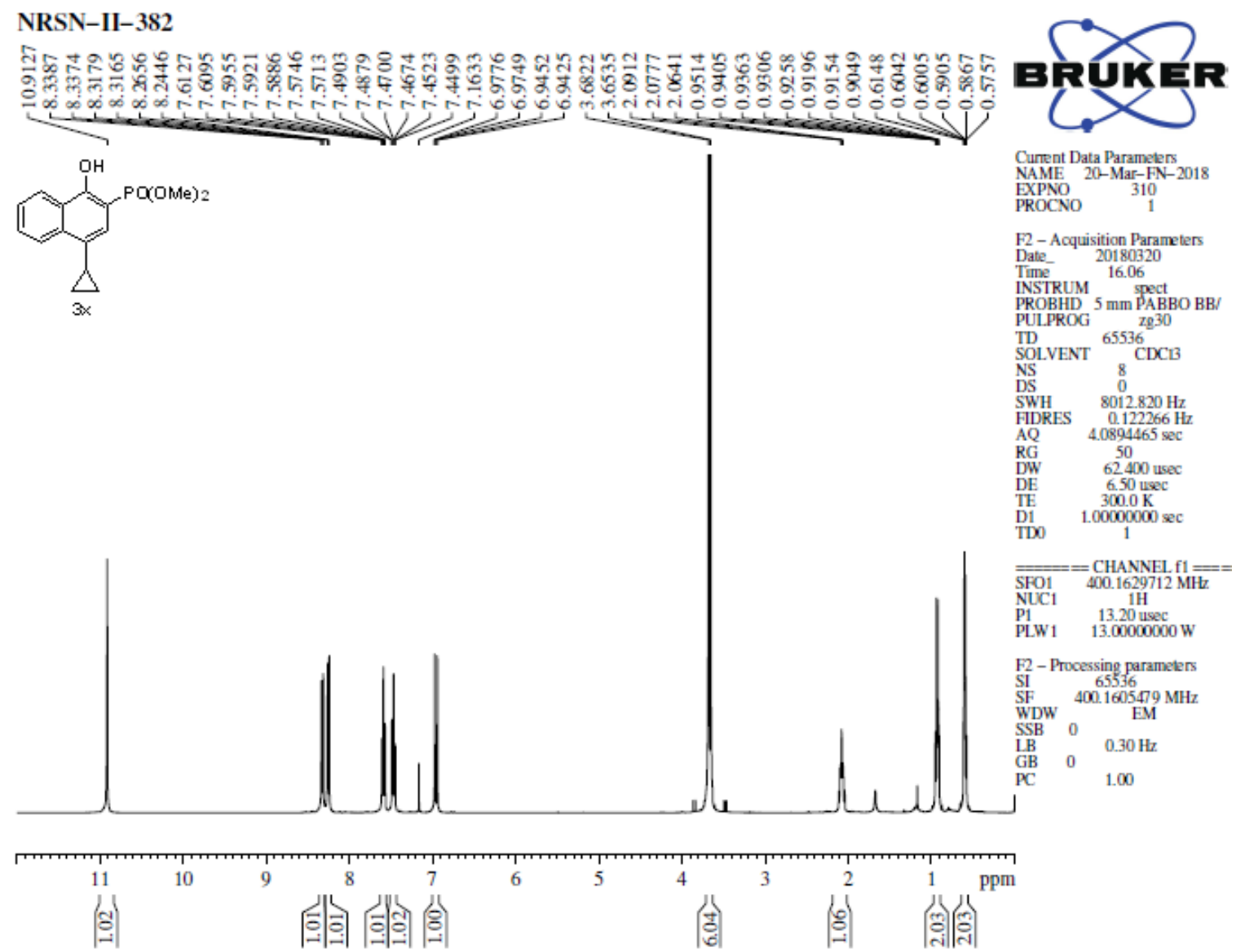

Spectrum 73: ${ }^{1} \mathrm{H}$ NMR spectrum of $\mathbf{3 x}$

NRSN II 382
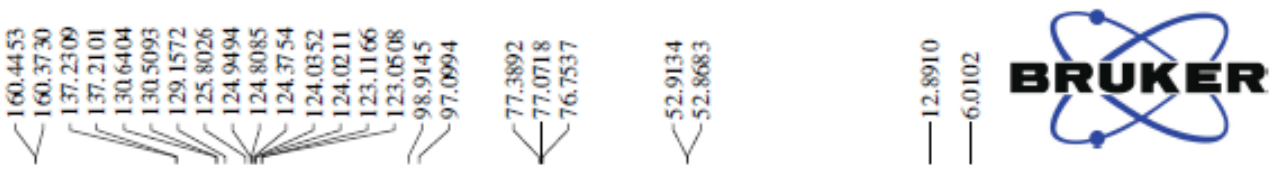

Current Data Paramekrs
NAME EXPNO

F2- Acquisition Paranetris

Date_ 20180323

Time 1.45

INSTRUM
PROBHD $5 \mathrm{~mm}$ PABBO BB

PULPROG z zpge 30

SOLVENT 65536

NS

$\begin{array}{lc}\text { NS } & 2048 \\ \text { DS } & 0\end{array}$

$\begin{array}{lc}\text { DS } & 0 \\ \text { SWH } & 24038.461 \mathrm{~Hz} \\ \text { FIDRES } & 0.366798 \mathrm{~Hz}\end{array}$

AQ 1.3631488

DW 20.800 usee

6.50 usec

$300.0 \mathrm{~K}$

$200000000 \mathrm{sec}$

Di1 $0.03000000 \mathrm{se}$

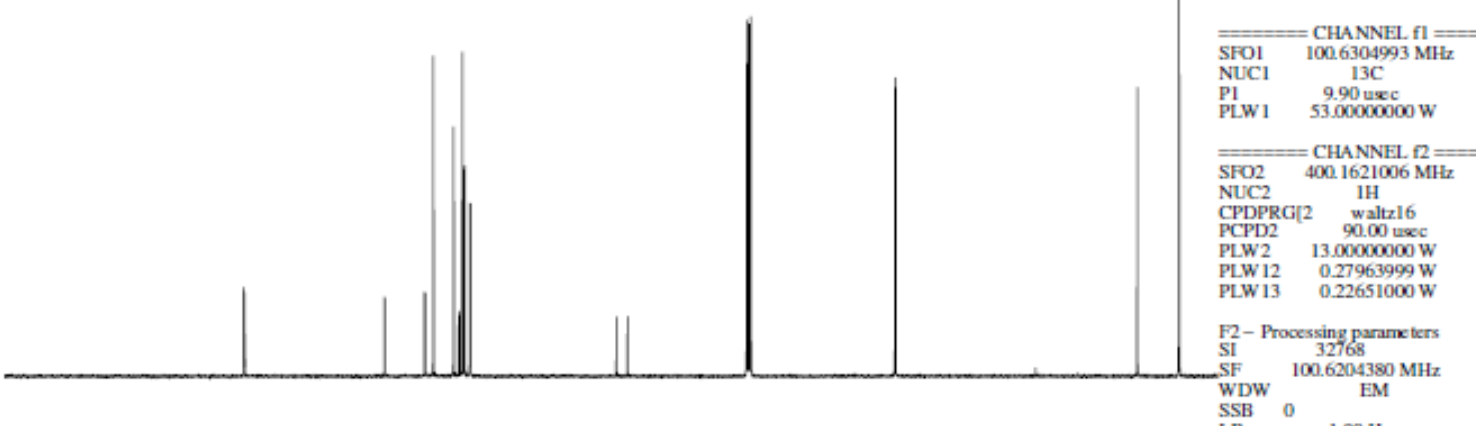

\begin{tabular}{lllllllllllllllllll|}
190 & 180 & 170 & 160 & 150 & 140 & 130 & 120 & 110 & 100 & 90 & 80 & 70 & 60 & 50 & 40 & 30 & 20 & Ppm
\end{tabular}

Spectrum 74: ${ }^{13} \mathrm{C}$ NMR spectrum of $\mathbf{3} \mathbf{x}$ 


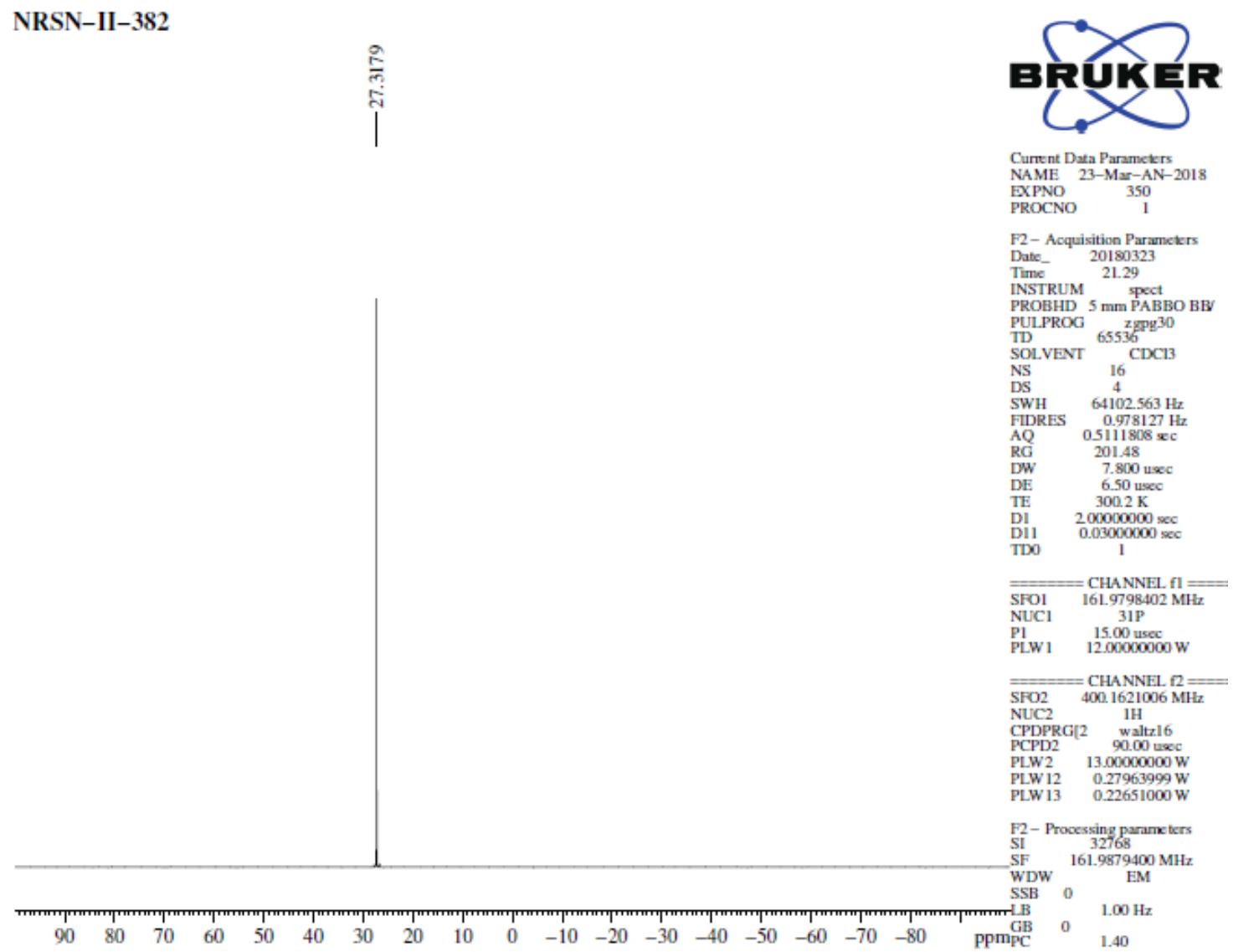

Spectrum 75: ${ }^{31} \mathrm{P}$ NMR spectrum of $\mathbf{3 x}$

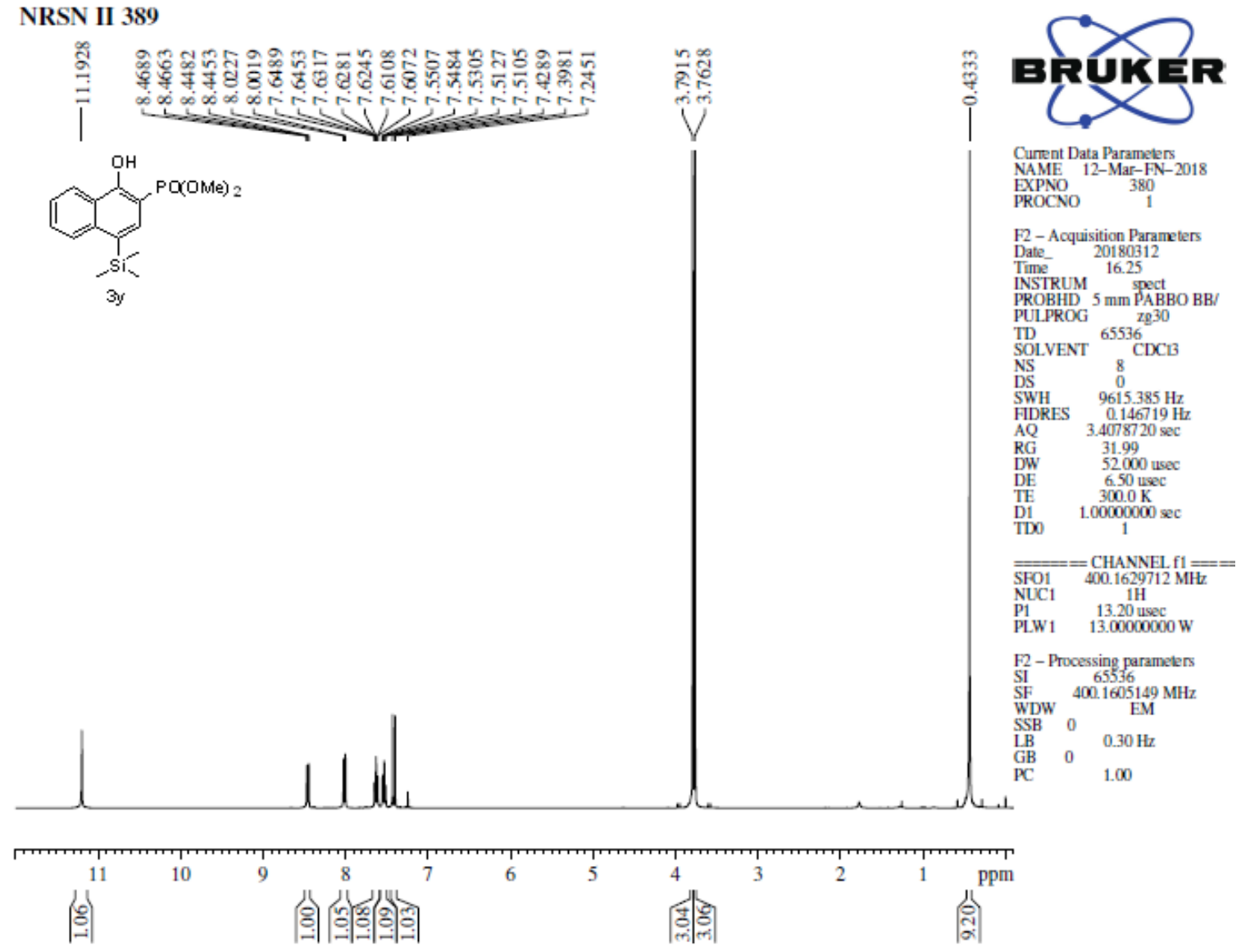

Spectrum 76: ${ }^{1} \mathrm{H}$ NMR spectrum of $\mathbf{3 y}$ 
NRSN-II-389

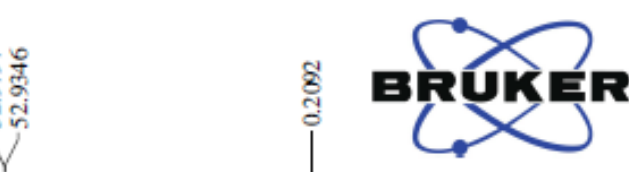

Current Data Paramekers
NAME 14-Mar-AN-2018 EXPNO

PROCNO

F2- Acquisitition Parancers

Date_ 2018031

TimsTRUM 4.32

PROBHD $5 \mathrm{~mm}$ PABBO BE

PULPROG $2 \mathrm{zpg}^{30}$

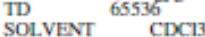

NS 2048

$\begin{array}{lc}\text { NS } & 2048 \\ \text { DS } & 4 \\ \text { SWH } & 24038.4611\end{array}$

FDRRES $\quad 0366798$ H

AQ $1.3631488 \mathrm{xc}$

RG 201.48

DE 6.50 usec

$300.0 \mathrm{~K}$

200000000

DI1 $0.030000000 \mathrm{sec}$

TDO

$=$ CHANNEL $\mathrm{fl}=$

SFOI $100.6304993 \mathrm{MHz}$

NUCl $\quad 13 \mathrm{C}$

P1 $\quad 9.90$ uxec
PLW1 53,00000000 W

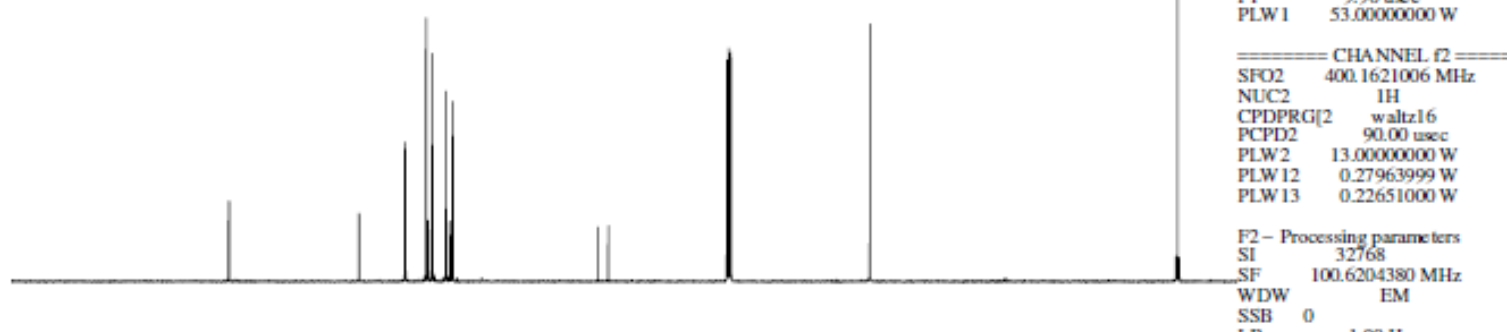

$1.00 \mathrm{~Hz}$

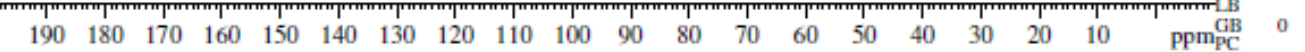

$\begin{array}{llllllllllllllllllllll}190 & 180 & 170 & 160 & 150 & 140 & 130 & 120 & 110 & 100 & 90 & 80 & 70 & 60 & 50 & 40 & 30 & 20 & 10 & \mathrm{ppm}_{\mathrm{PC}}^{\mathrm{GB}} & 0 & 1.40\end{array}$

Spectrum 77: ${ }^{13} \mathrm{C}$ NMR spectrum of $\mathbf{3 y}$

NRSN-II-389
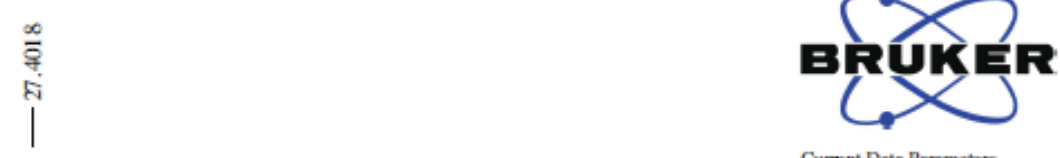

Current Data Parameters

NAME 16-Mar-AN-201 EXPNO
PROCNO

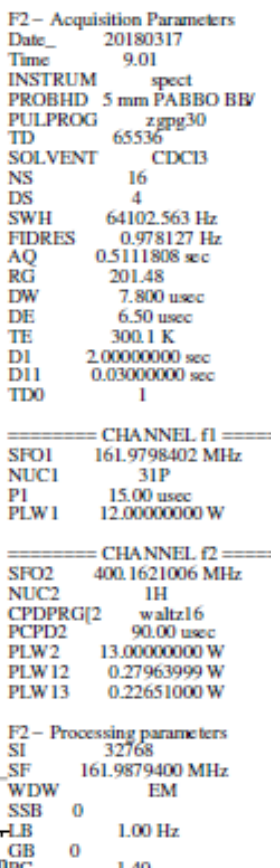

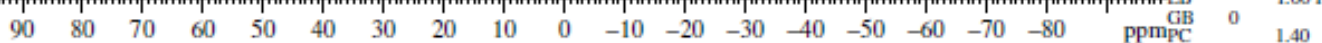

Spectrum 78: ${ }^{31} \mathrm{P}$ NMR spectrum of $\mathbf{3 y}$ 


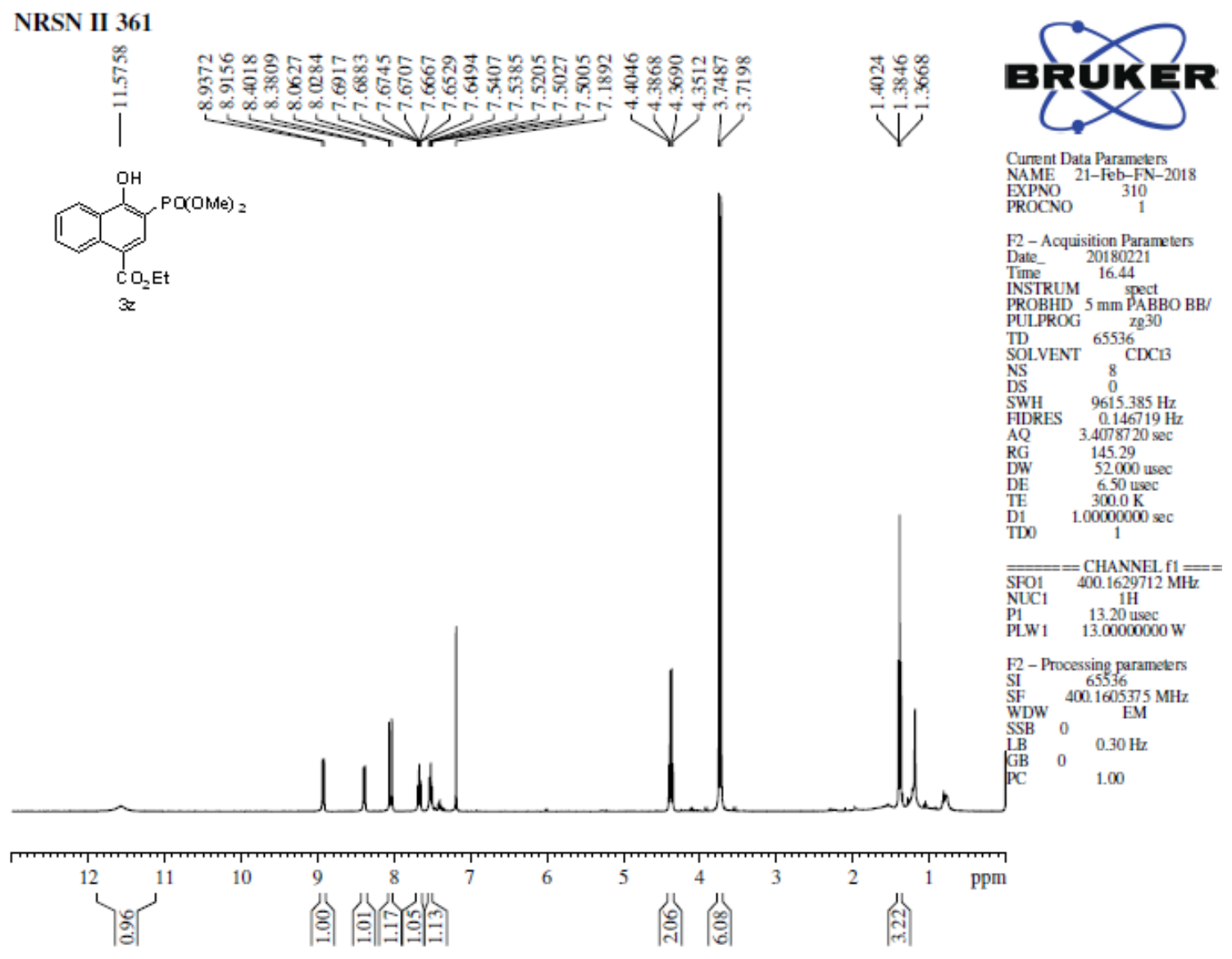

Spectrum 79: ${ }^{1} \mathrm{H}$ NMR spectrum of $\mathbf{3 z}$

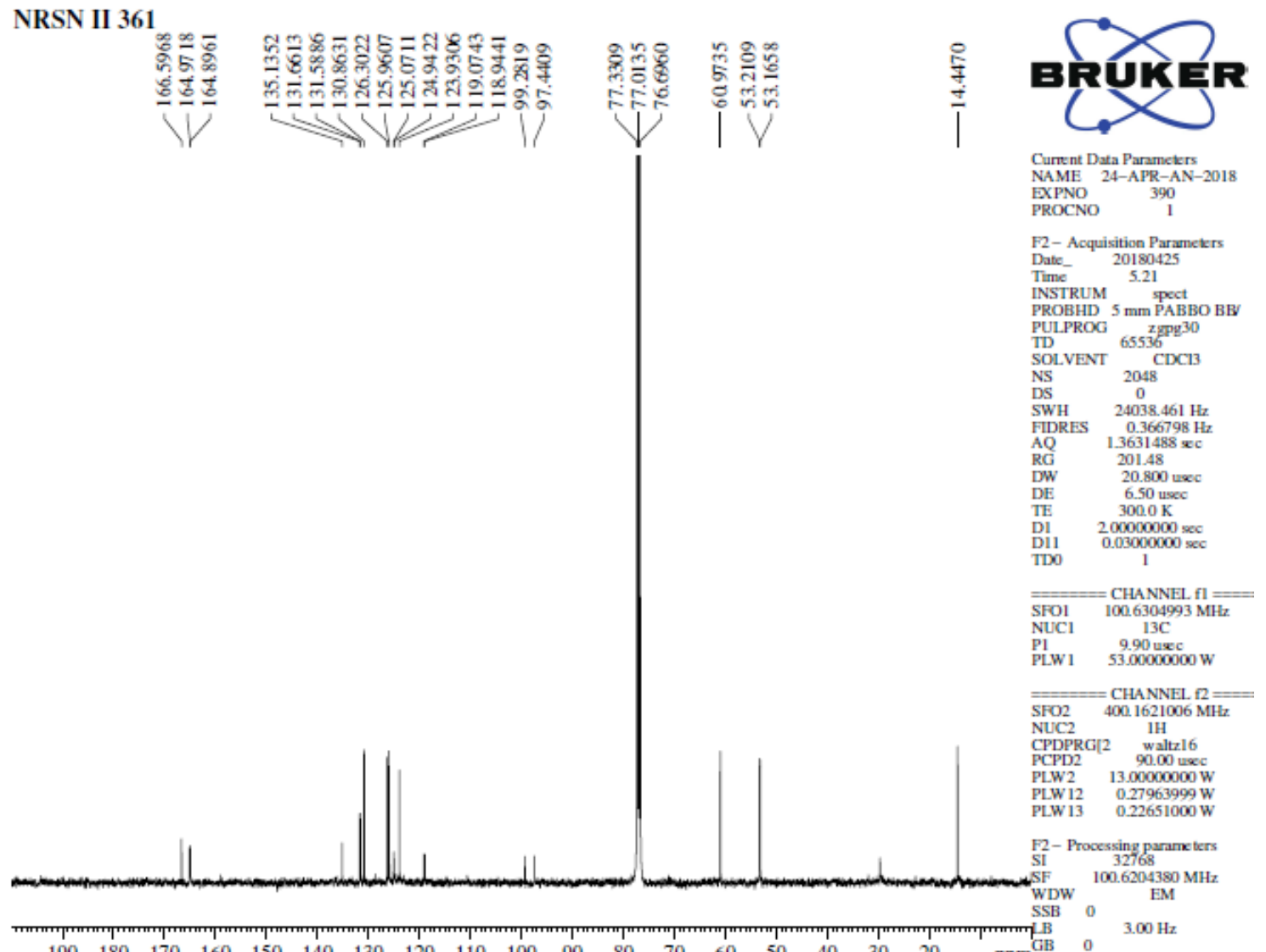

\begin{tabular}{llllllllllllllllllll}
190 & 180 & 170 & 160 & 150 & 140 & 130 & 120 & 110 & 100 & 90 & 80 & 70 & 60 & 50 & 40 & 30 & 20 & $\mathrm{Ppm}$ & 0 \\
\hline $\mathrm{PC}$ & 0 & 1.40
\end{tabular}

Spectrum 80: ${ }^{13} \mathrm{C}$ NMR spectrum of $\mathbf{3 z}$ 


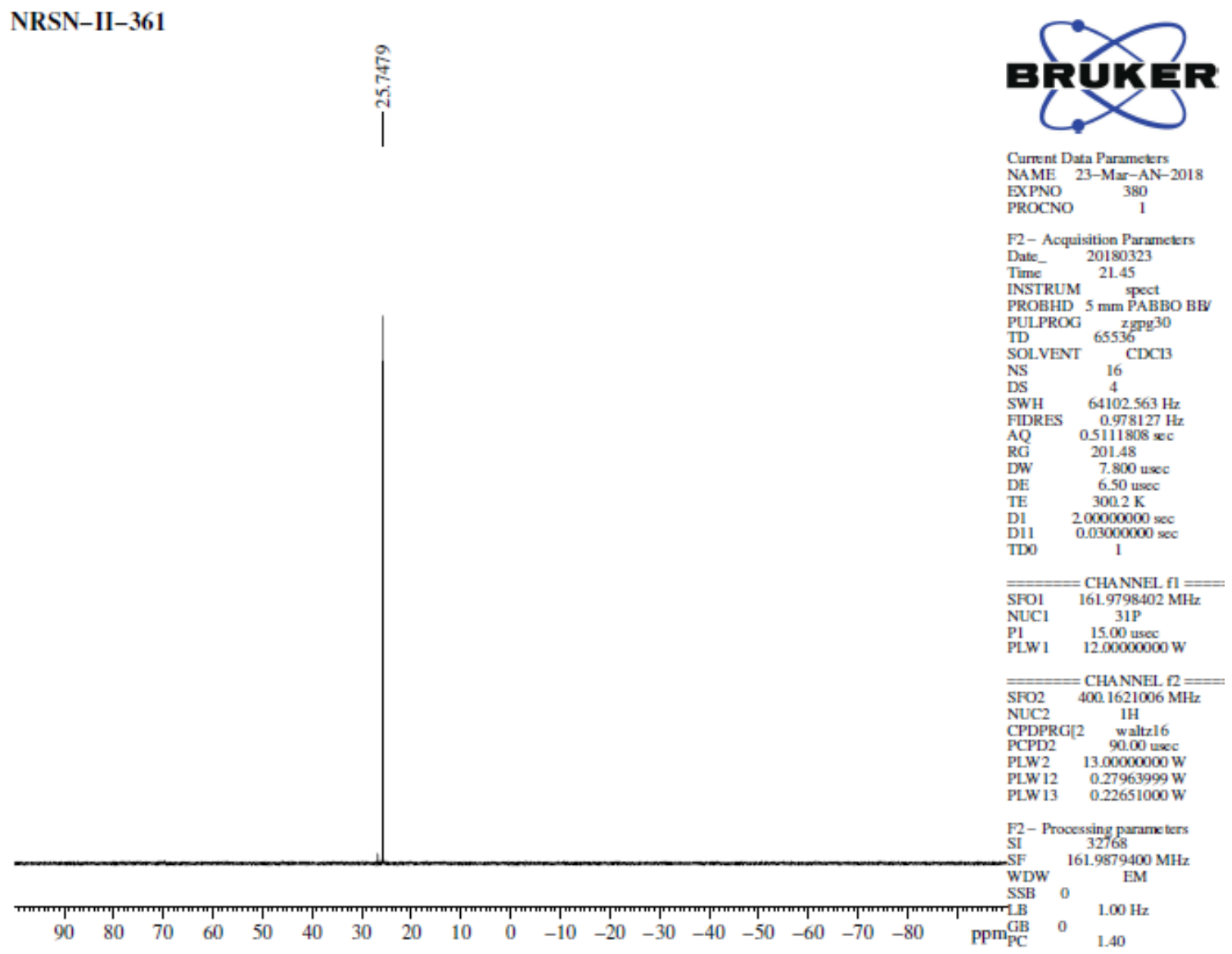

Spectrum 81: ${ }^{31} \mathrm{P}$ NMR spectrum of $\mathbf{3 z}$

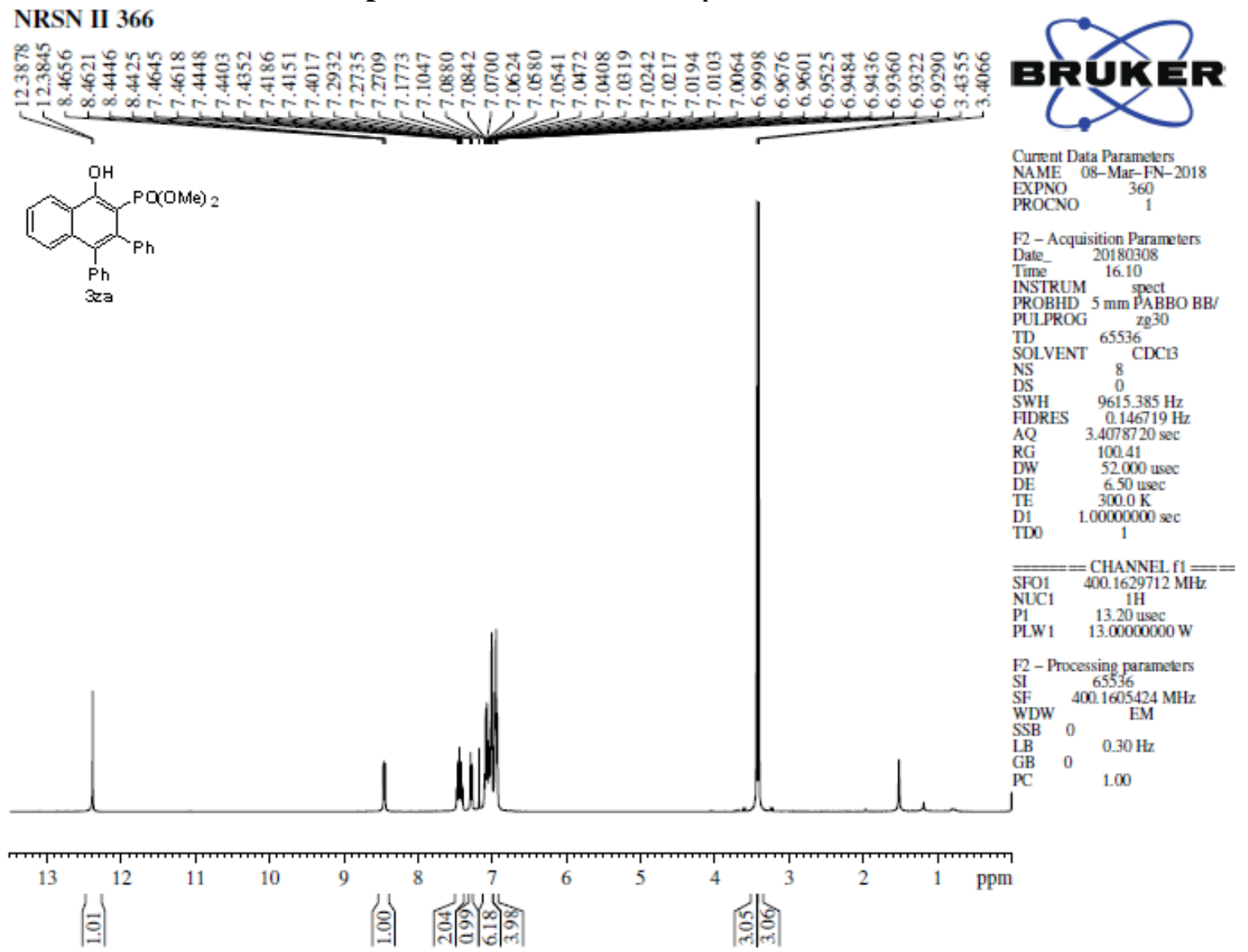

Spectrum 82: ${ }^{1} \mathrm{H}$ NMR spectrum of $\mathbf{3 z a}$ 
NRSN II 366

Current Data Paramekers

EXPNO 390

PROCNO

F2- Acquisition Parameters

Date__ 20180312

Time

18.52

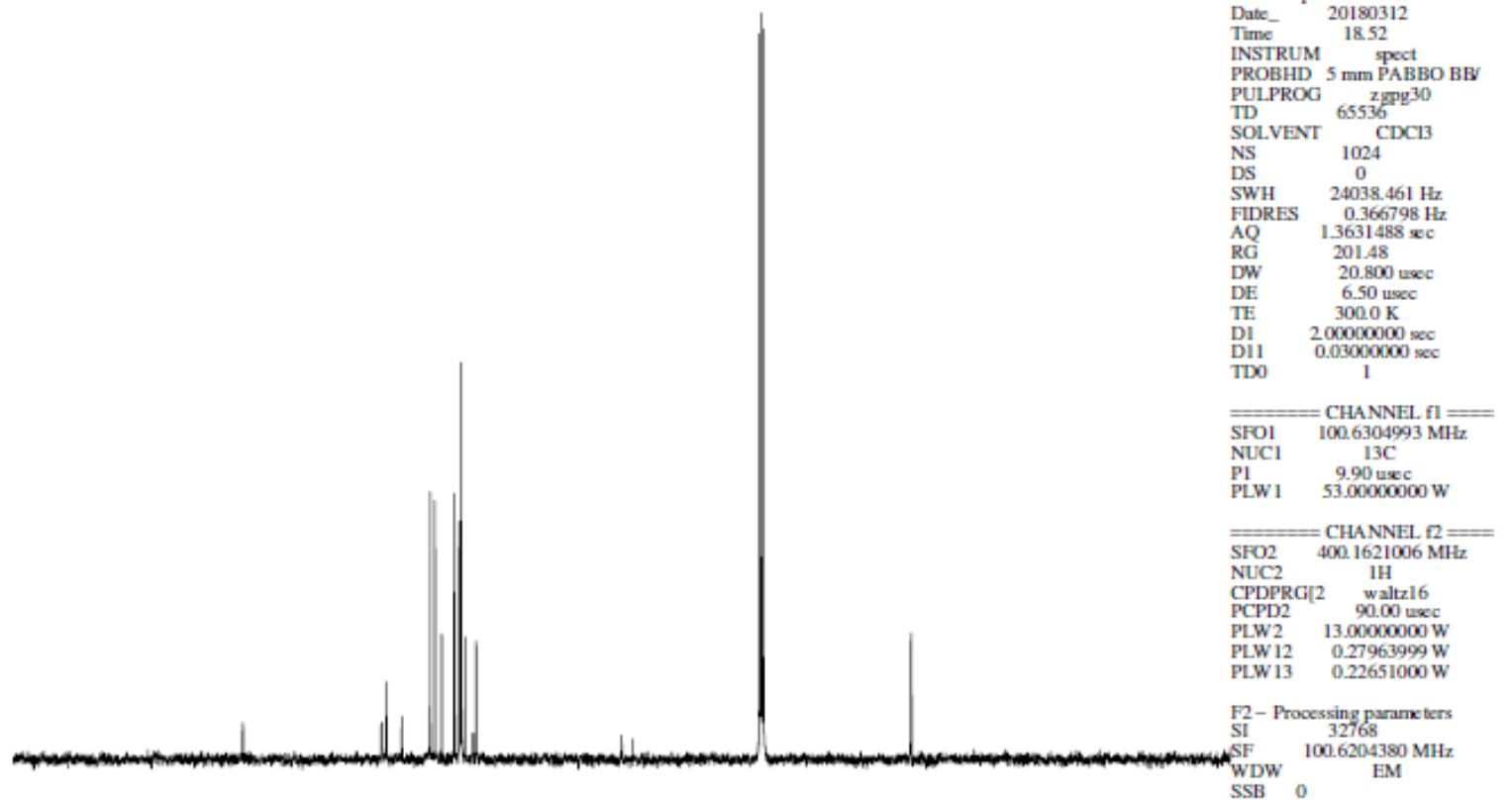

PULPROG ${ }^{2} \mathrm{zpg}^{30}$

$\mathrm{CDC} 13$

DS

$461 \mathrm{~Hz}$

1

FOl $1006304993 \mathrm{MHz}$

NuCl $13 \mathrm{C}$

PLW1 53.00000000 W

$=$ CHANNEL $\mathbb{R}=$

NUC2 400.1621006

waltzl

0.22651000 W

F2- Processing parame ter

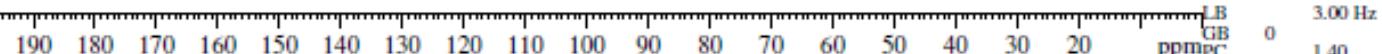

$\begin{array}{lllllllllllllllllllll}190 & 180 & 170 & 160 & 150 & 140 & 130 & 120 & 110 & 100 & 90 & 80 & 70 & 60 & 50 & 40 & 30 & 20 & \text { ppmPC } & 0 & 1.40\end{array}$

Spectrum 83: ${ }^{13} \mathrm{C}$ NMR spectrum of 3za

NRSN-II-366
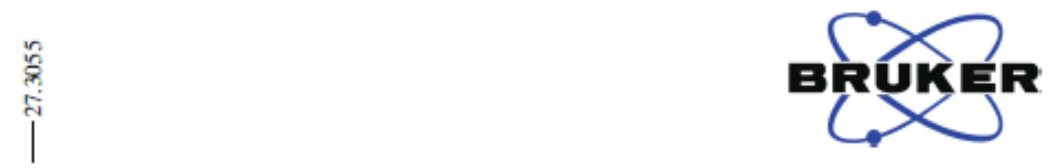

Current Data Paramelers

NAME 13-Mar-AN-2018

$\begin{array}{lrl}\text { EXPNO } & 310 \\ \text { PROCNO } & 1\end{array}$

F2- Acquisition Parameters

Date_ 20180313

Time 2253

PROBHD 5 mm PABBO BB

PULPROG $2 \mathrm{zpg}^{30}$

SOLVENT $\quad$ CDCl3

NS 16

NS

SWH $\quad 64102.563 \mathrm{~Hz}$

FIDRES $\quad 0.978127 \mathrm{~Hz}$

AQ $0.5111808 \times$

$\begin{array}{ll}\mathrm{RG} & 201.48 \\ \mathrm{DW} & 7.800 \mathrm{usec}\end{array}$

$\begin{array}{ll}\text { DE } & 6.50 \text { usce } \\ \text { TE } & 3002 \mathrm{~K}\end{array}$

DI 200000000

DI1 $0.03000000 \mathrm{sec}$

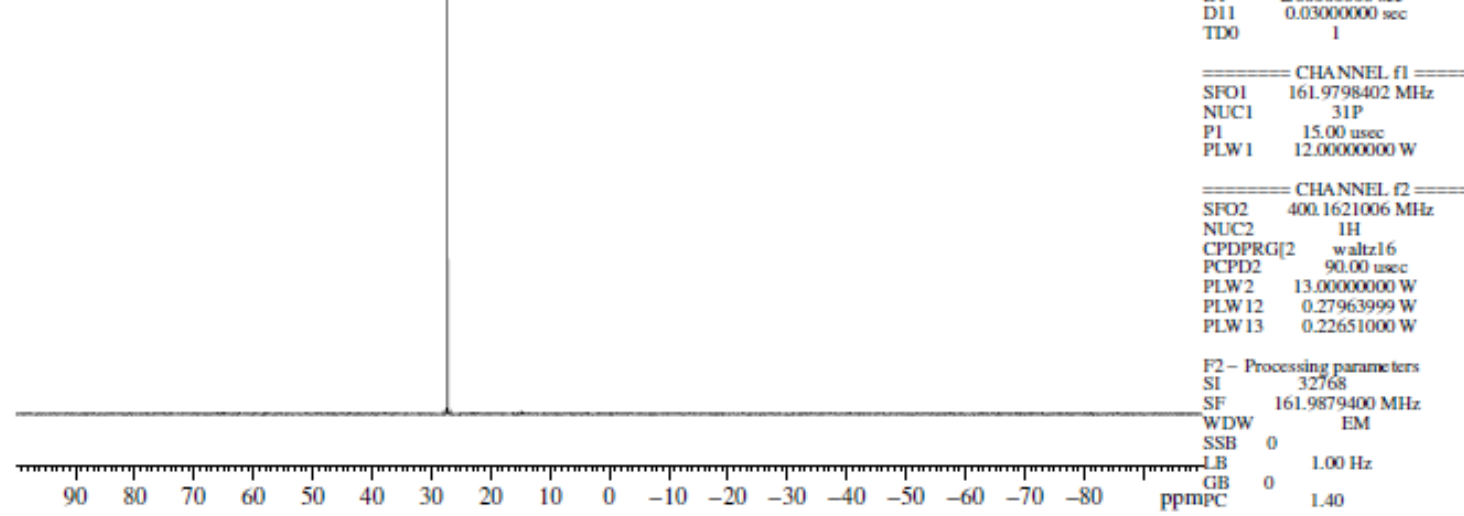

$\begin{array}{lllllllllllllllllllll}90 & 80 & 70 & 60 & 50 & 40 & 30 & 20 & 10 & 0 & -10 & -20 & -30 & -40 & -50 & -60 & -70 & -80 & \text { ppmpC } & 0 & 1.40\end{array}$

Spectrum 84: ${ }^{31} \mathrm{P}$ NMR spectrum of 3za 


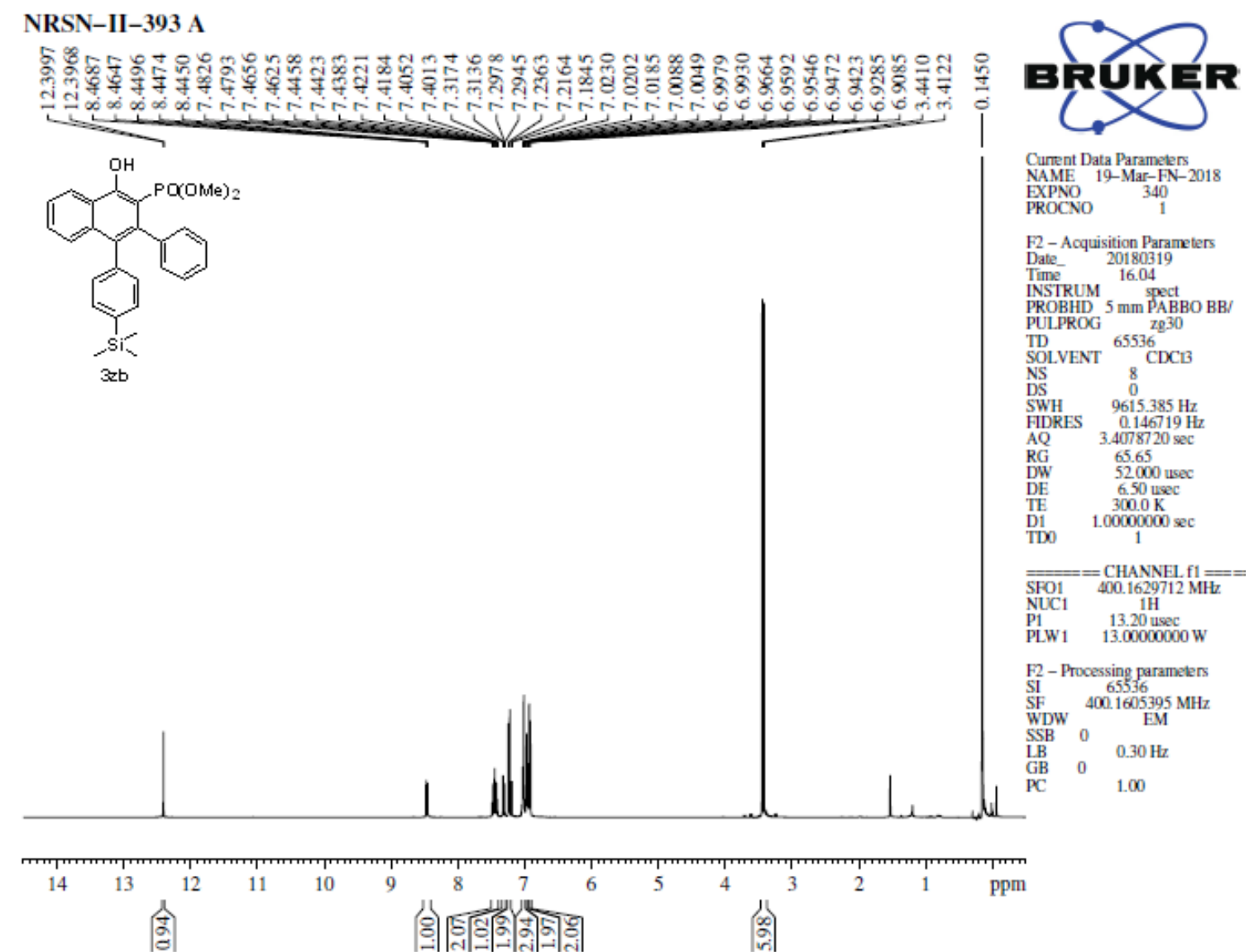

Spectrum 85: ${ }^{1} \mathrm{H}$ NMR spectrum of $\mathbf{3 z b}$

NRSN-II-393A
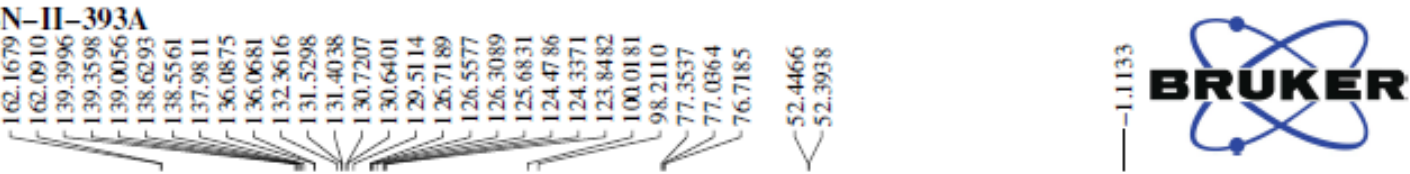

Current Data Parameters
NAME 21-MAR-AN-2018 EXPNO

F2- Acquisition Parameters

Date_ 20180322

INSTRUM 9.10 spect

PROBHD $5 \mathrm{~mm}$ PABBO BE

PULPROG $2 \mathrm{z}^{2} \mathrm{Pg}^{30}$

SOLVENT ${ }^{65536} \mathrm{CDC13}$

$\begin{array}{lc}\text { NS } & 2048 \\ \text { DS } & 0 \\ \text { SWH } & 24038.461 \mathrm{~Hz}\end{array}$

FIDRES $\quad 0.366798 \mathrm{H}$

AQ $\quad 1.3631488 \times$

DW $\quad 20.800$ used

TE $3000 \mathrm{~K}$

$\begin{array}{ll}\text { DI } & 200000000 \mathrm{sec} \\ \text { DII } & 0.030000000 \mathrm{sec}\end{array}$

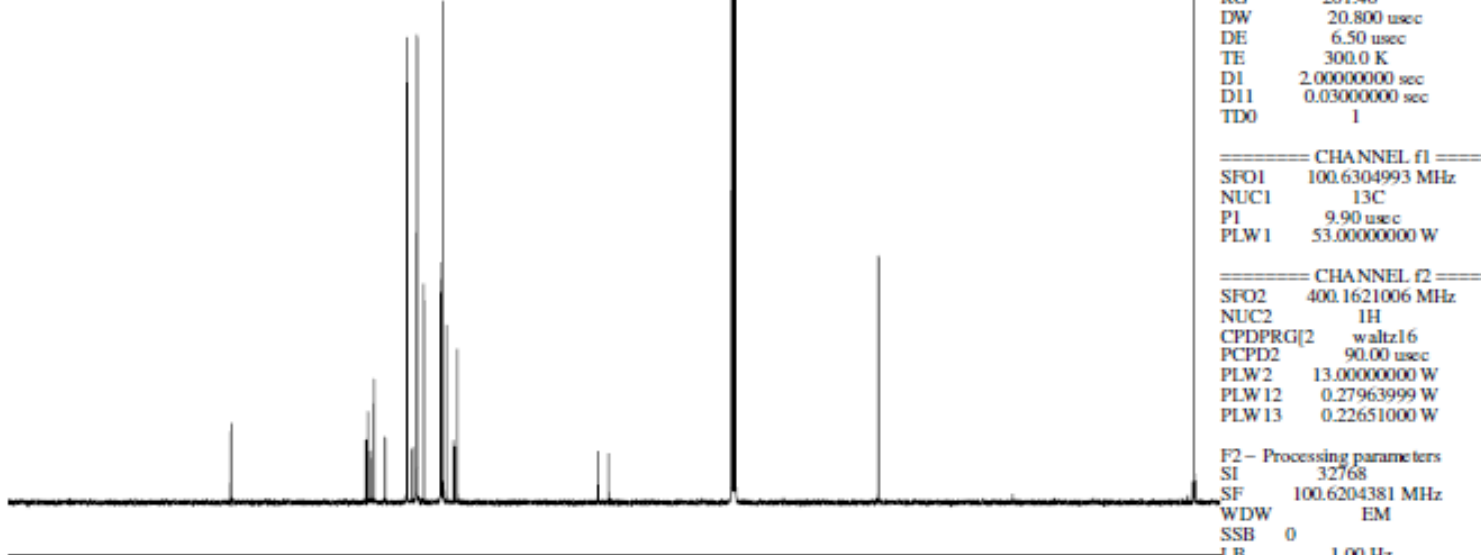

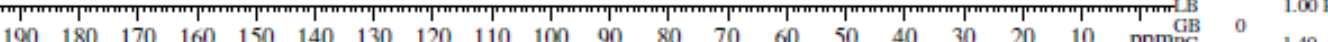

Spectrum 86: ${ }^{13} \mathrm{C}$ NMR spectrum of $\mathbf{3 z b}$ 
NRSN-II-393-A

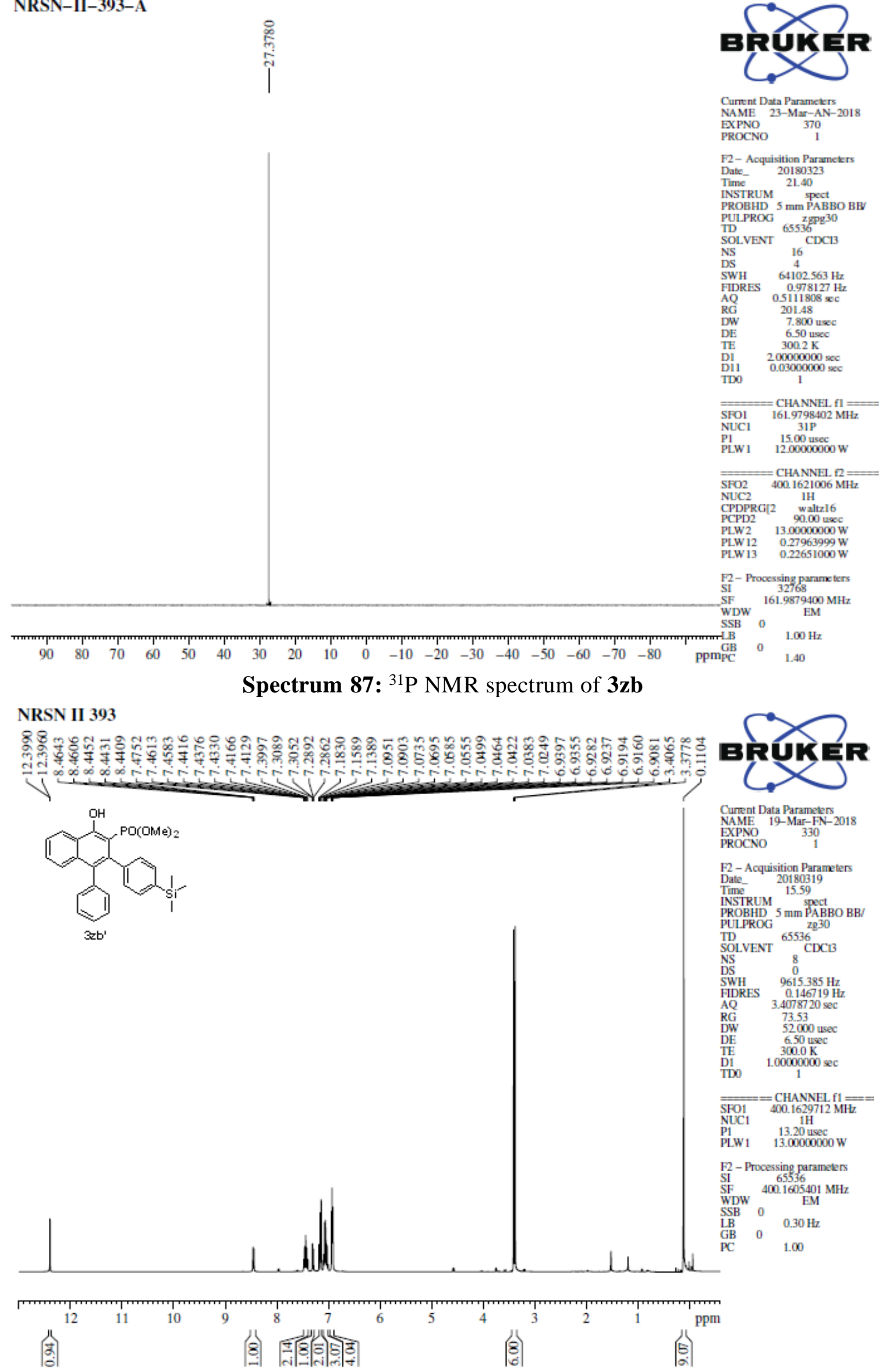

Spectrum 88: ${ }^{1} \mathrm{H}$ NMR spectrum of $\mathbf{3 z b}$ ' 
Time 7.09

INSTRUM
PROBHD 5 mm PABBO BB

PULPRO $5 \mathrm{~mm}$ PABBO

SOLVENT $\quad{ }^{65536}$ CDC13

NS 2048

$\begin{array}{ll}\text { DS } & 0 \\ \text { SWH } & 24038.461 \mathrm{~Hz}\end{array}$

$\begin{array}{lr}\text { SWH } & 24038.461 \mathrm{~Hz} \\ \text { FIDRES } & 0.36798 \mathrm{H}\end{array}$

$\begin{array}{lc}\mathrm{AQ} & 1.3631488 \\ \mathrm{RG} & 201.48\end{array}$

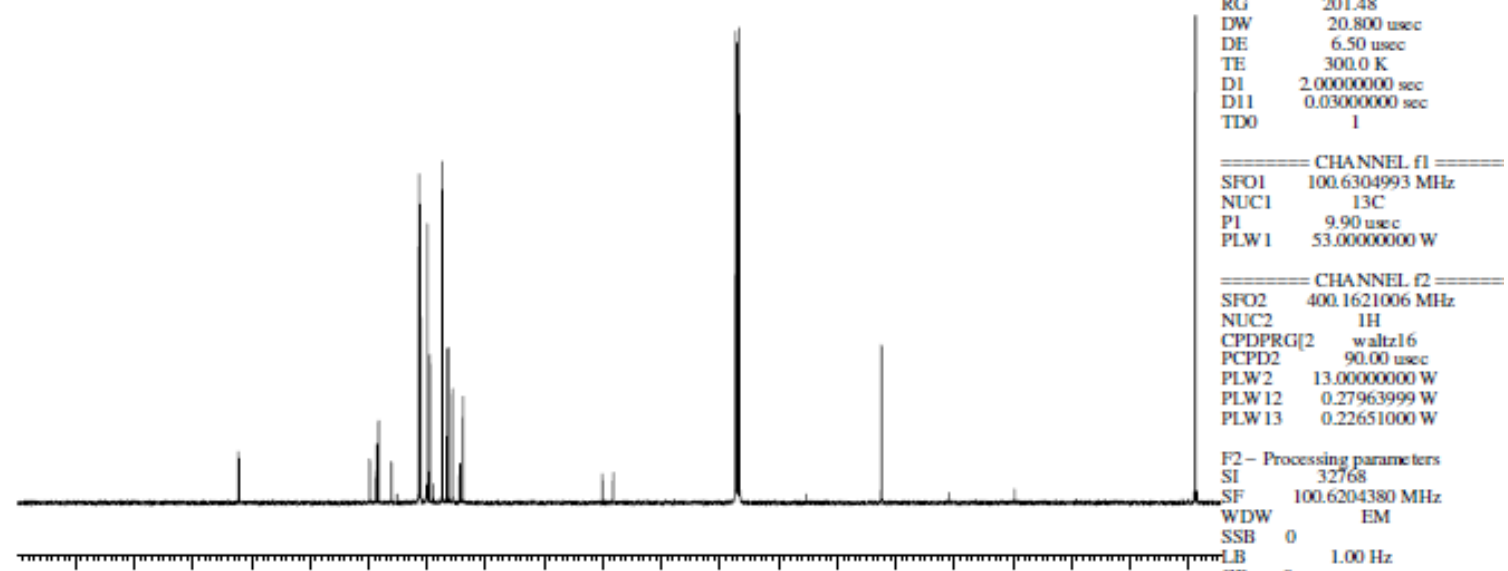

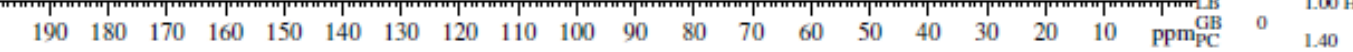

Spectrum 89: ${ }^{13} \mathrm{C}$ NMR spectrum of $\mathbf{3 z b}$ '

NRSN-II-393

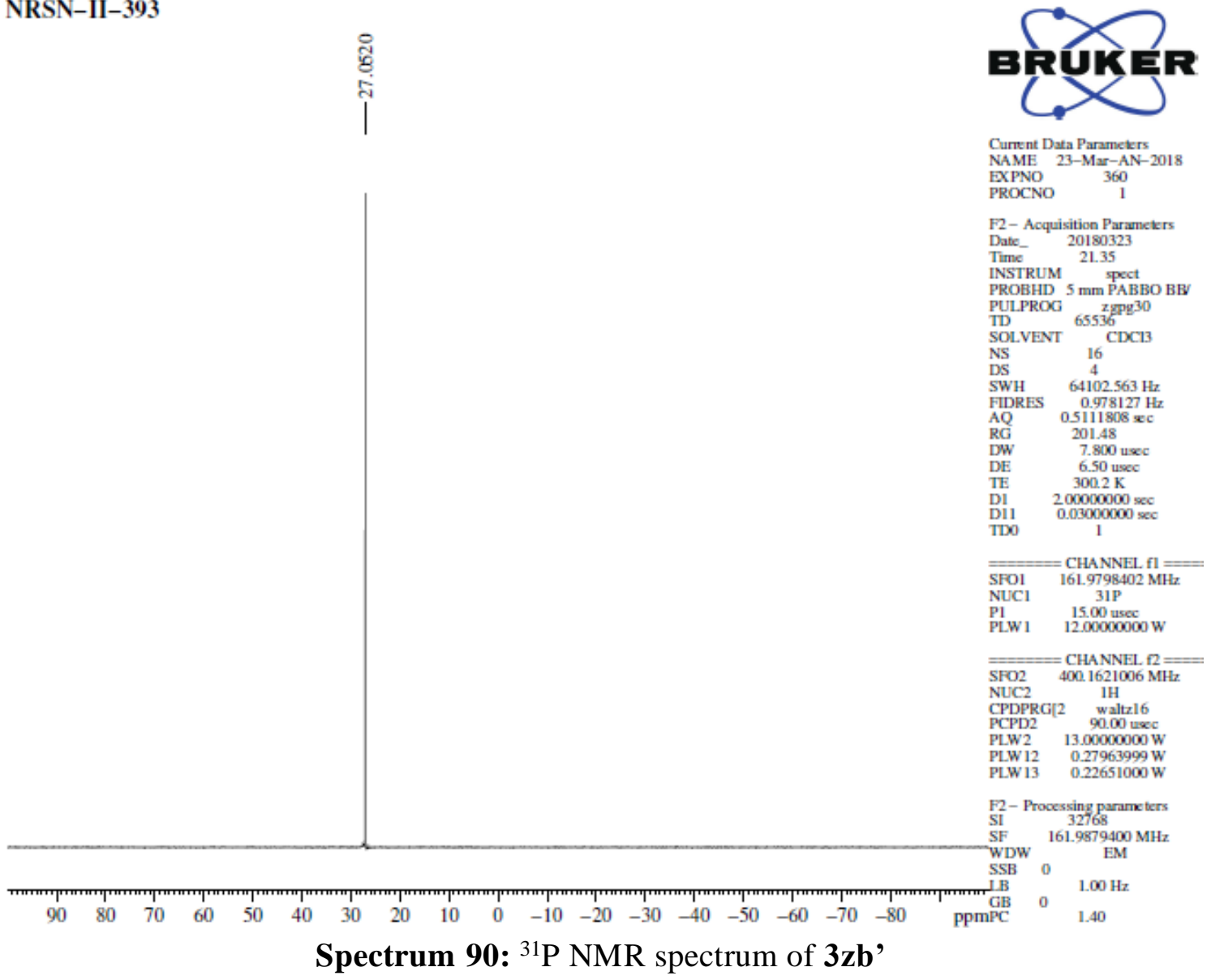




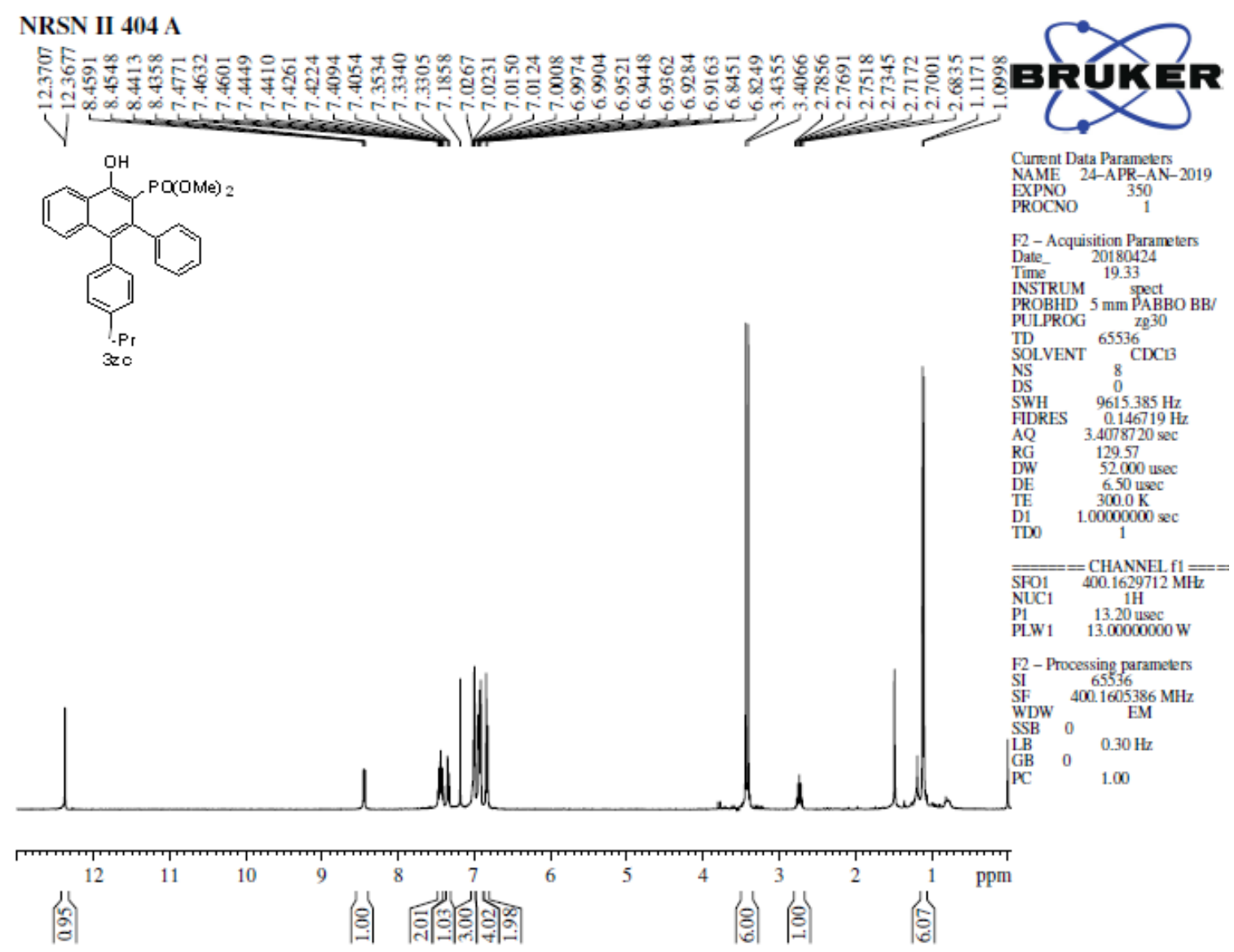

Spectrum 91: ${ }^{1} \mathrm{H}$ NMR spectrum of 3zc

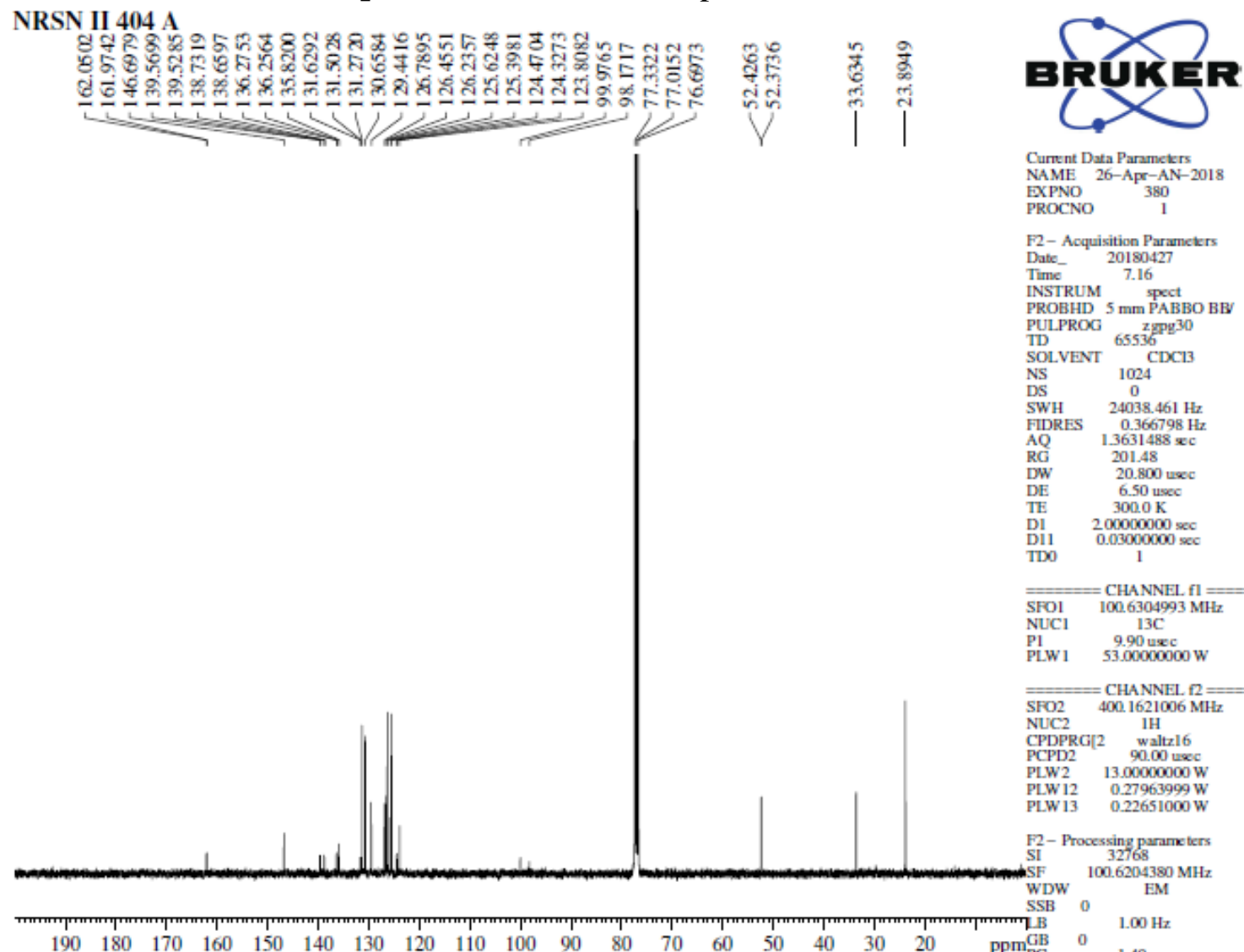

Spectrum 92: ${ }^{13} \mathrm{C}$ NMR spectrum of $3 z c$ 


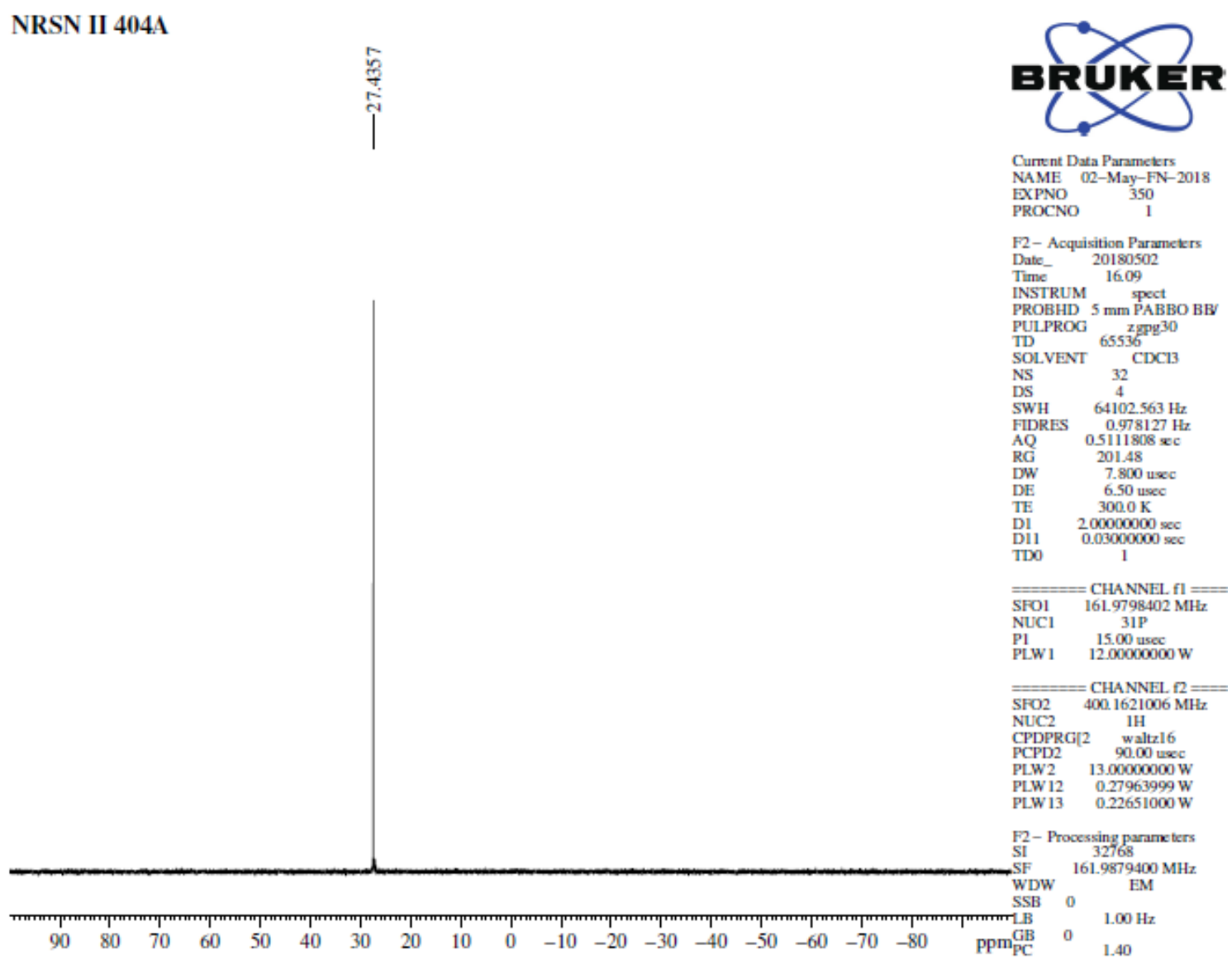

Spectrum 93: ${ }^{31} \mathrm{P}$ NMR spectrum of $\mathbf{3 z c}$

\section{NRSN II 404}

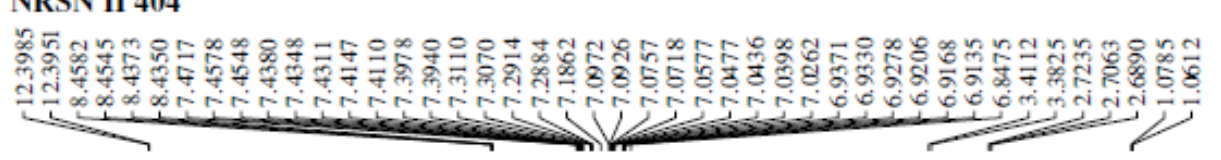

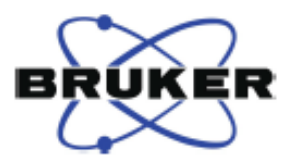<smiles>CC(=O)Oc1c(-c2ccc(C(C)C)cc2)c(-c2ccccc2)c2ccccc2c1O</smiles>

Cunent Data Paramelers 2018 EXPNO

PROCNO

F2-Acquisition Parameters

$\begin{array}{lc}\text { Date_- } & 20180424 \\ \text { Time } & 19.28\end{array}$

INSTRUM 19.28 spect

PROBHD 5 mm PABBO

$\begin{array}{ll}\text { PUL.PROG } & \mathrm{zg} 30 \\ \text { TD } & 65536\end{array}$

$3 z$

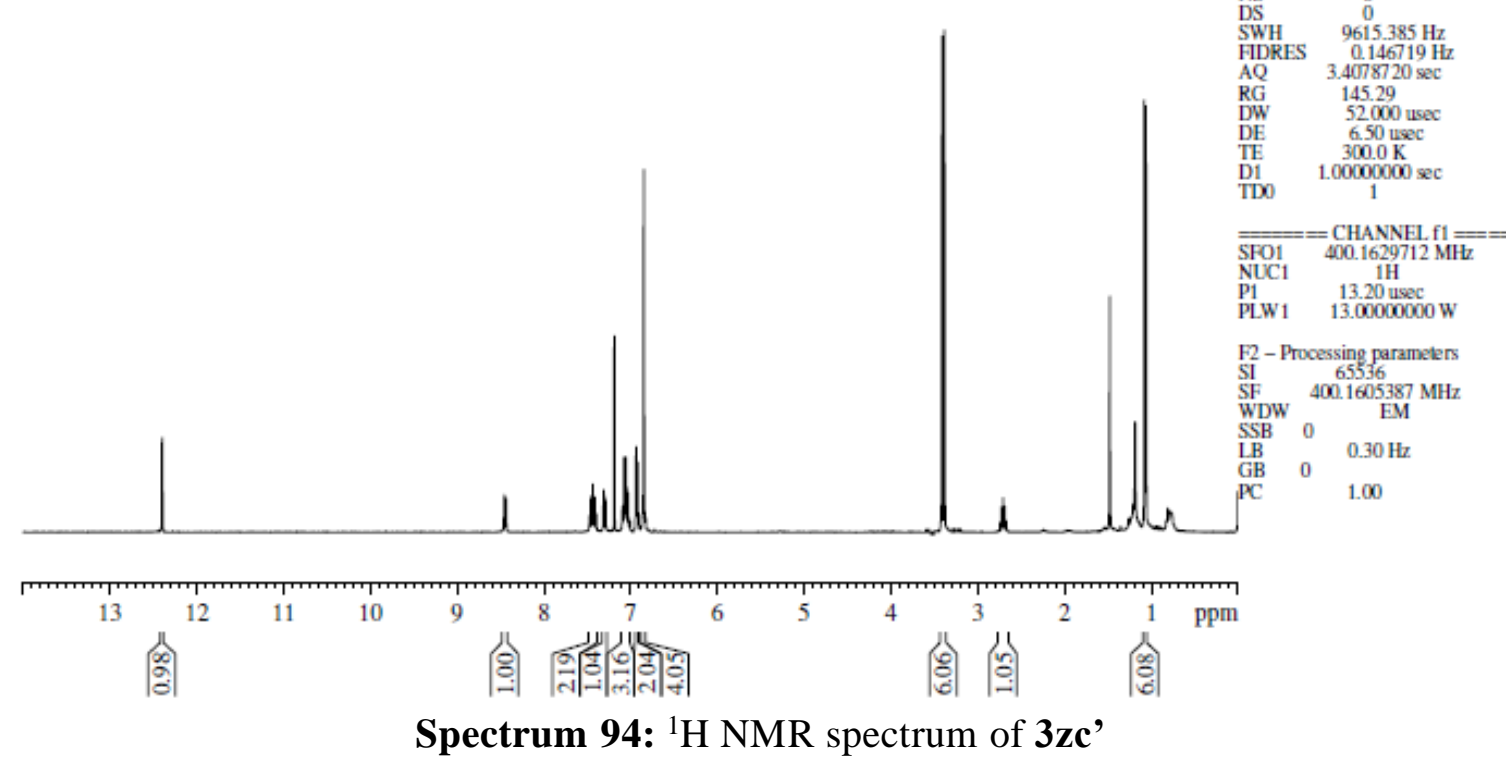




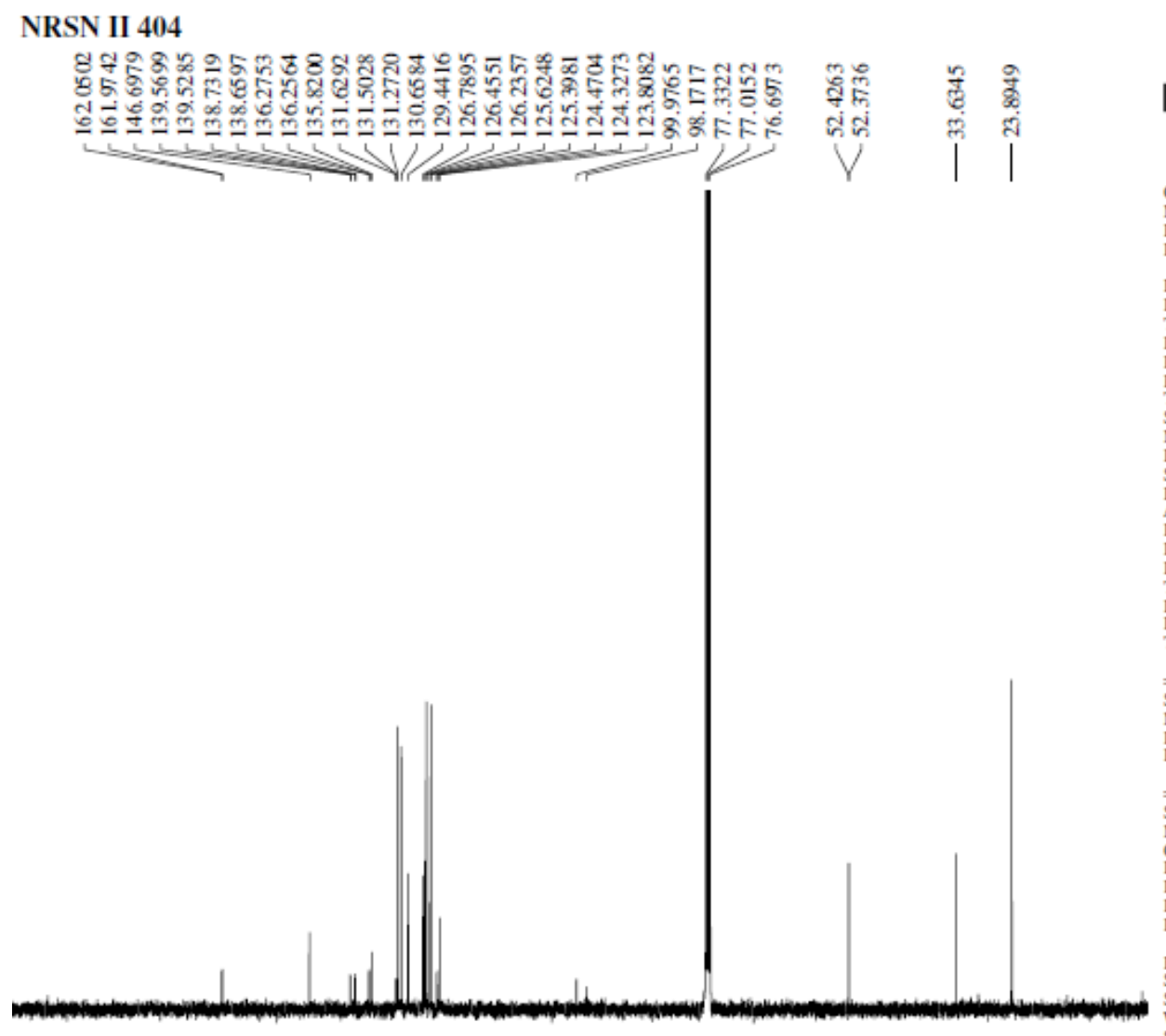

BRिERR

Current Data Parameters
NAME
26-APp-AN-2018

PROCNO

F2- Acquisition Parameters

Dabe_ 20180427

Time

7.16

PROBHD $5 \mathrm{~mm}$ PABBO BR

PULPROG $2 \mathrm{zPg}^{30}$

TD $6553 \%$

NS 1024

$\begin{array}{ll}\text { DS } & 0 \\ \text { SWH } & 24038.461 \mathrm{~Hz}\end{array}$

$\begin{array}{lr}\text { SWH } & 24038.461 \mathrm{~Hz} \\ \text { FIDRES } & 0.366798 \mathrm{~Hz} \\ \text { AO } & 13631488 \times c\end{array}$

RG $\quad 201.48$

DW $\quad 20.800$ usec

6.50 usee

200000000

DI1 $0.03000000 \mathrm{sec}$

SFOI $=$ CHANNEL $\mathrm{fl}=$

NUCl $\quad 13 \mathrm{C}$

$\begin{array}{lc}\text { P1 } & 9.90 \text { uxec } \\ \text { PLW1 } & 53.00000000 \mathrm{~W}\end{array}$

$=$ CHANNEL $R=$

S602.1621000

NUC2 $1 \mathrm{H}$

CPDPRG12 waltz16

PLW2 $13.00000000 \mathrm{~W}$

PLW $12-0.27963999 \mathrm{~W}$

PLW $13 \quad 0.22651000 \mathrm{~W}$

FI- Processing parame ters

$\begin{array}{ll}\mathrm{SI} & 32768 \\ \mathrm{SF} & 100.6204380 \mathrm{MH}\end{array}$

WDW EM

$\begin{array}{lll}\text { SSB } & 0 \\ \mathrm{LB} & 100 \mathrm{~Hz}\end{array}$

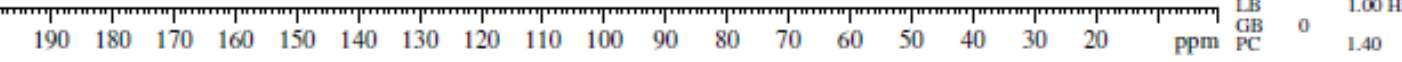

Spectrum 95: ${ }^{13} \mathrm{C}$ NMR spectrum of 3zc'

NRSN II 404
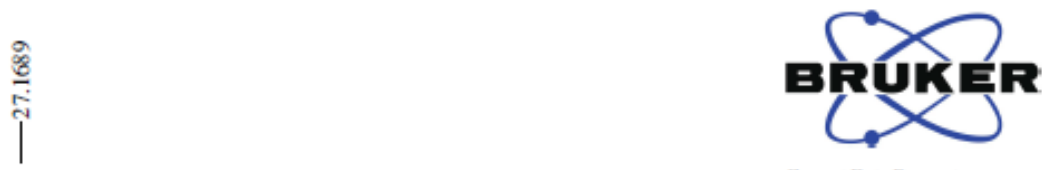

Current Data Parameters

NAME 02-May-FN-2018

EXPNO 340

F2- Acquisition Parameters

Date_ 20180502

Time

NSTRUM spect

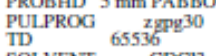

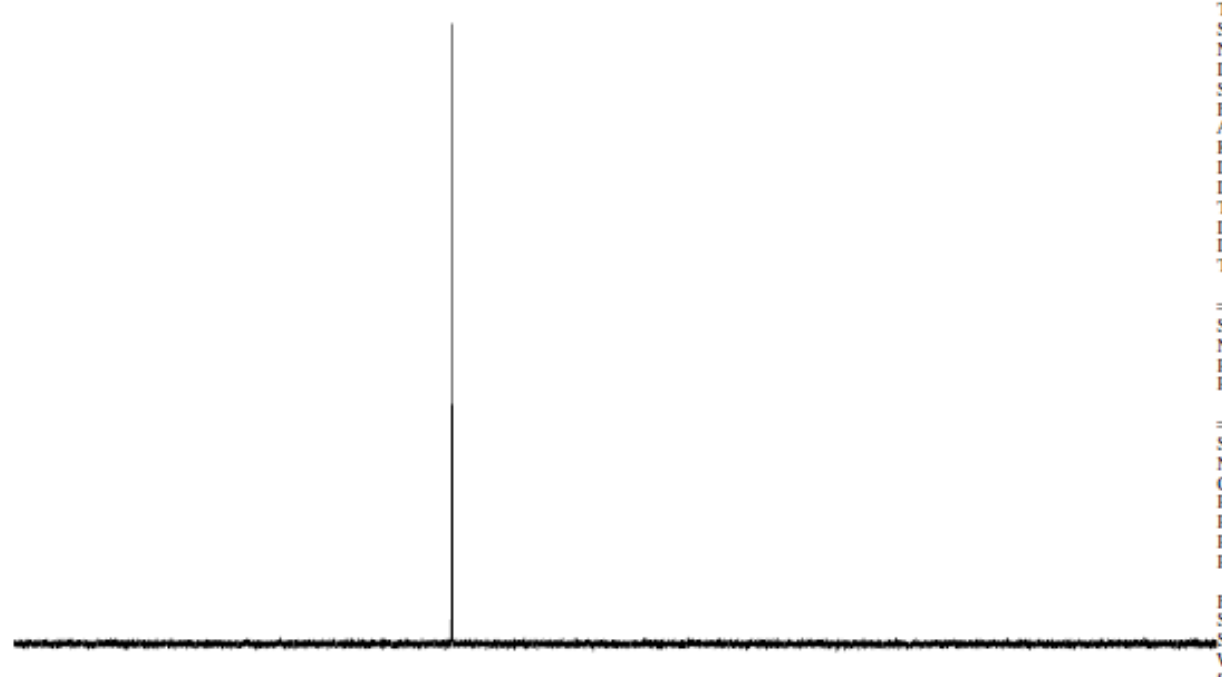

SOLVENT

Ds

SWH $\quad 64102.563 \mathrm{~Hz}$

FIDRES $0.978127 \mathrm{H}$

AQ $\quad 0.51118$

$\begin{array}{ll}\text { DW } & 7.800 \text { usec } \\ \text { DE } & 6.50 \text { usec }\end{array}$

6.50 use:

$200000000 \mathrm{sec}$

1

SFOI $=$ CHANNEL $\mathrm{fl}=$

SFOl 16L.9798402 MHz

NUCl 31 P

15.00 usec

CHANNEL $f 2=$

$\quad 400.1621006 \mathrm{M}$

CPDPRG[2 1H waltzl

90.00 usec

$\begin{array}{lr}\text { PLW 2 } & 13.00000000 \mathrm{~W} \\ \text { PLW 12 } & 0.27963999 \mathrm{~W}\end{array}$

Proessing parameters

SI - Processing paramk ters

$\begin{array}{lc}\text { SF } & 161.9879400 \mathrm{M} \\ \text { WDW } & \text { EM }\end{array}$

$\begin{array}{llllllllllllllllllllll}90 & 80 & 70 & 60 & 50 & 40 & 30 & 20 & 10 & 0 & -10 & -20 & -30 & -40 & -50 & -60 & -70 & -80 & \mathrm{Ppm}_{\mathrm{PC}}^{\mathrm{G}}\end{array}$

0

Spectrum 96: ${ }^{31} \mathrm{P}$ NMR spectrum of 3zc' 


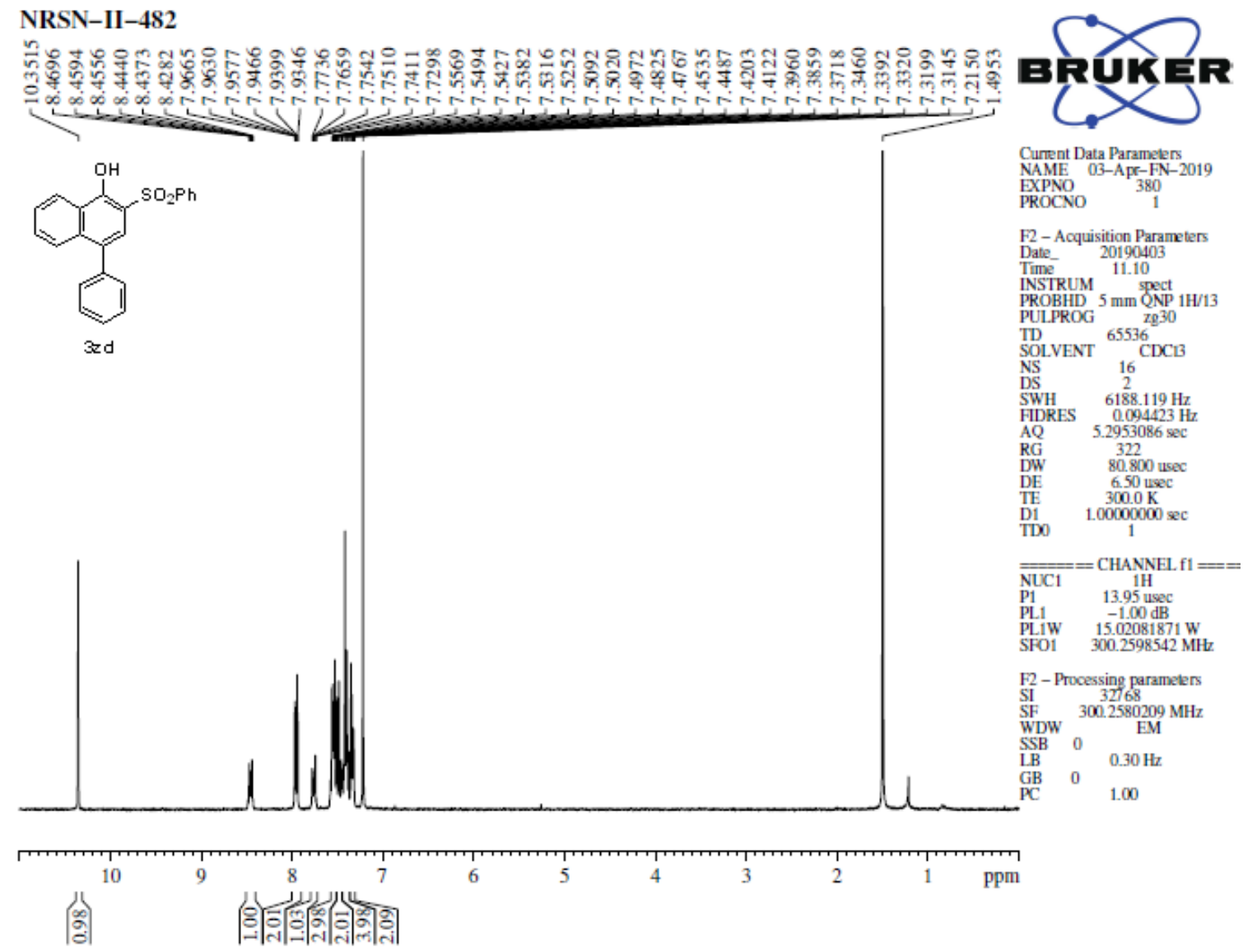

Spectrum 97: ${ }^{1} \mathrm{H}$ NMR spectrum of $\mathbf{3 z d}$
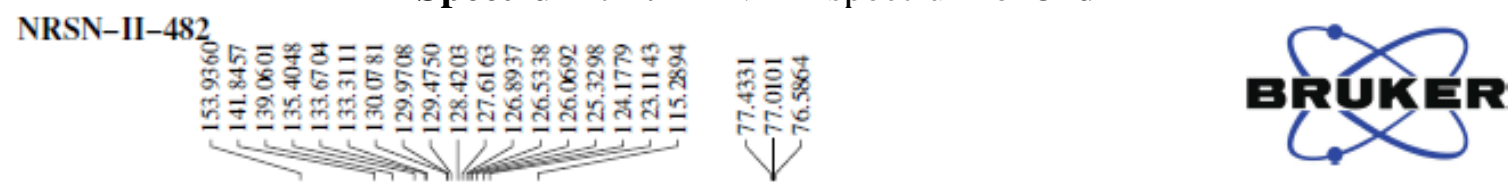

Curnent Data Parameters EXPNO $\quad 330$

F2- Processing parame ters
SI $\quad 32768$

$\begin{array}{lc}\text { SF } & 75.4999349 \mathrm{MHz} \\ \text { WDW } & \text { EM }\end{array}$

WSB 0 EM

$\begin{array}{lll}\mathrm{LB} & 0 & 1.00 \mathrm{H} \\ \mathrm{GB} & 0 & \end{array}$

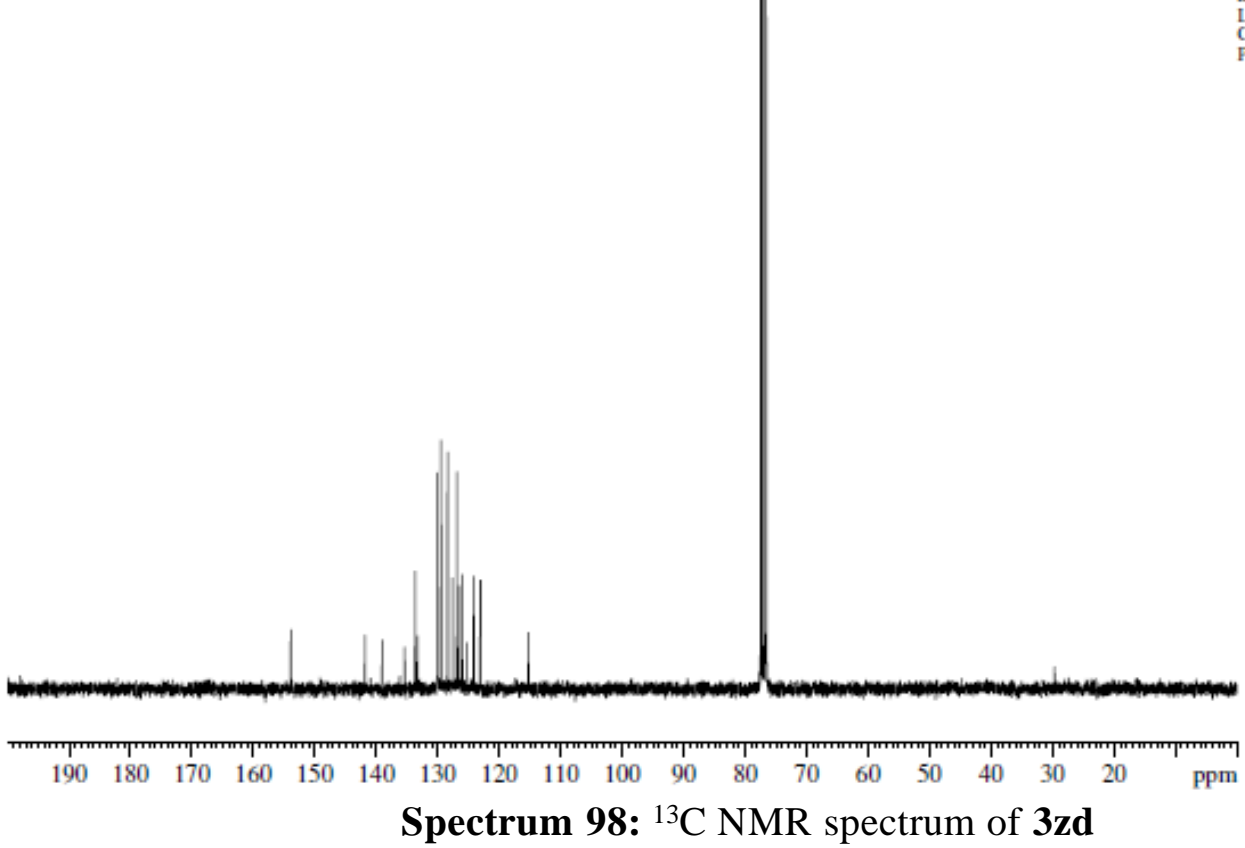




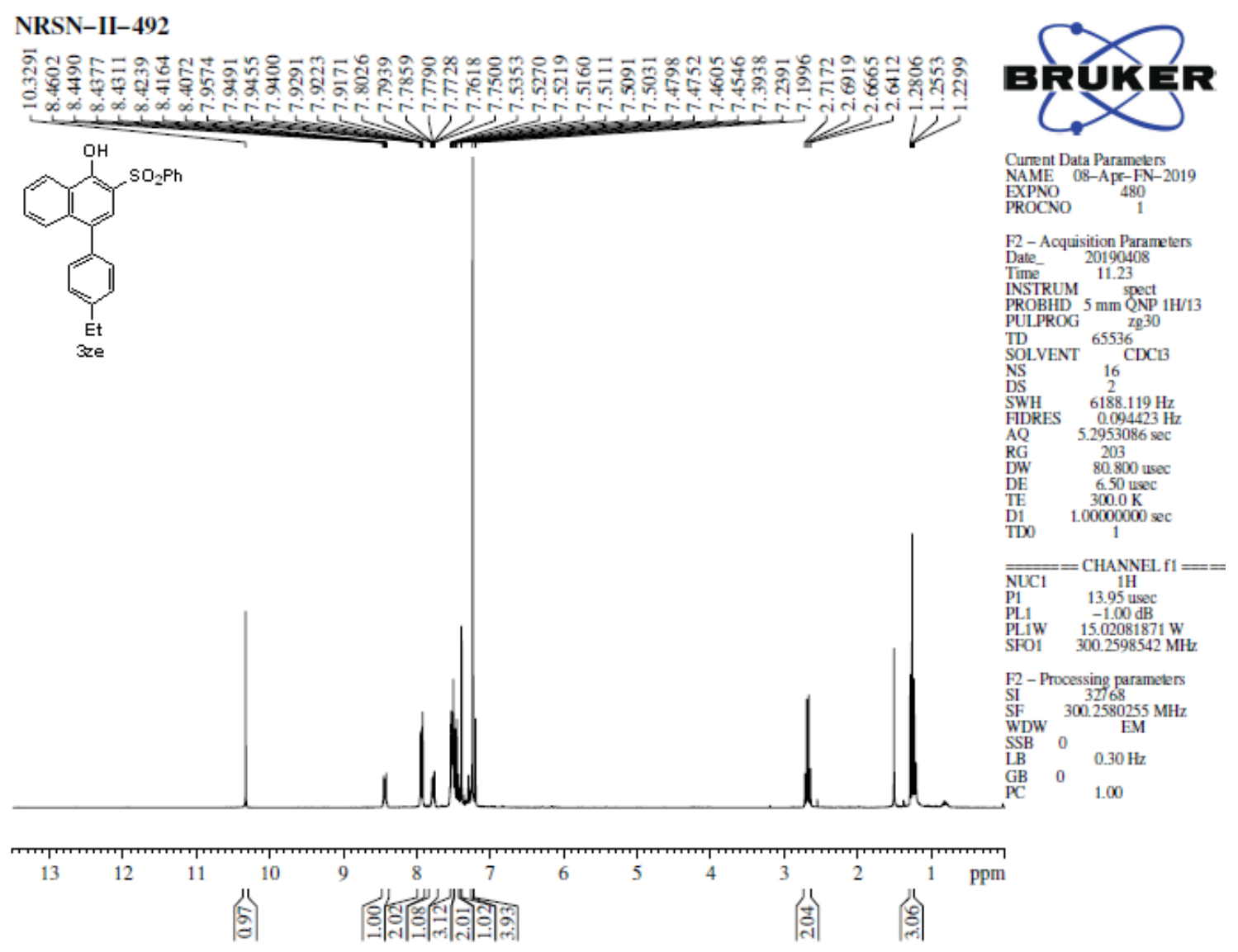

Spectrum 99: ${ }^{1} \mathrm{H}$ NMR spectrum of 3ze

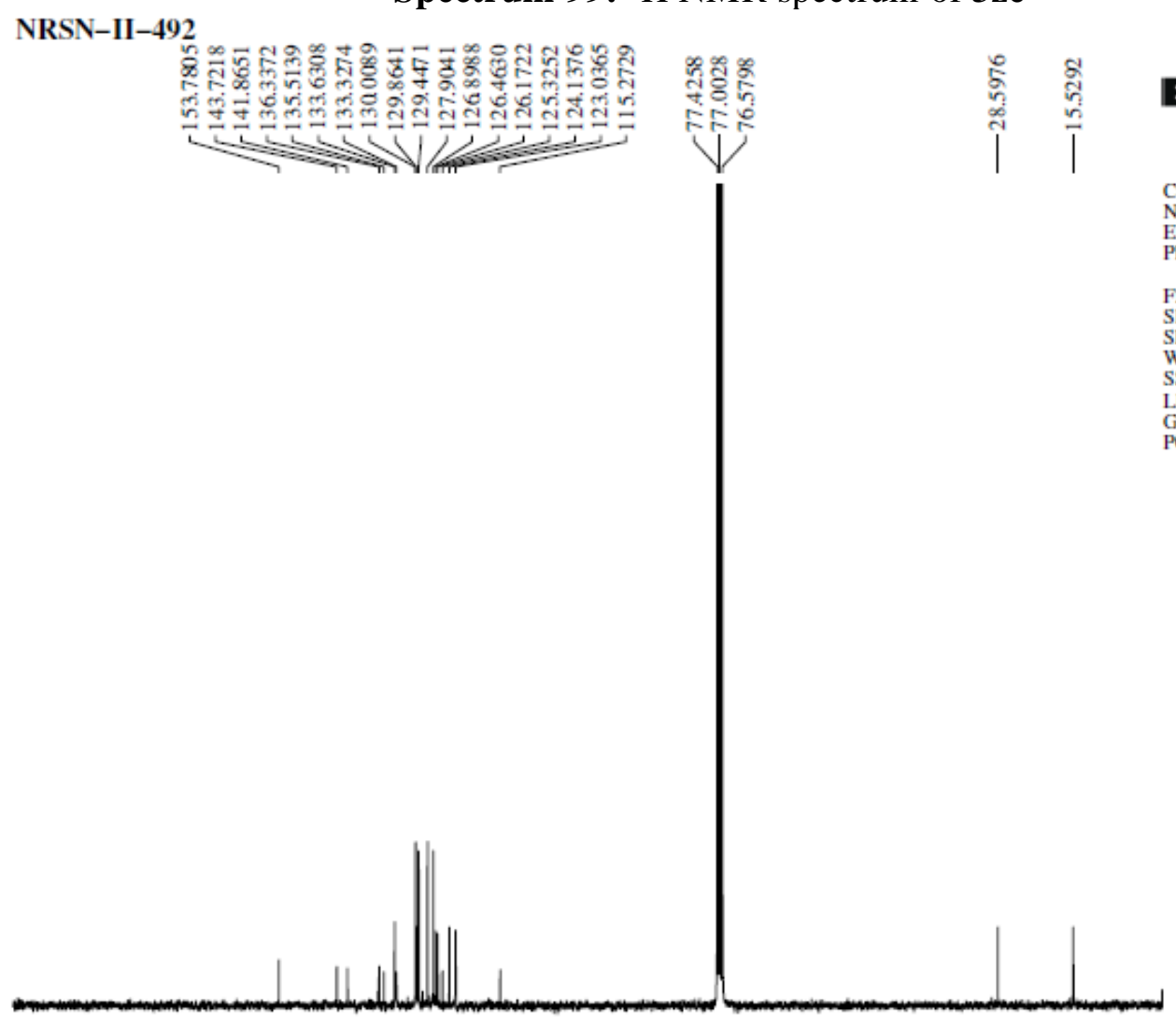

$\begin{array}{lllllllllllllllllll}190 & 180 & 170 & 160 & 150 & 140 & 130 & 120 & 110 & 100 & 90 & 80 & 70 & 60 & 50 & 40 & 30 & 20 & \mathrm{ppm}\end{array}$

Spectrum 100: ${ }^{13} \mathrm{C}$ NMR spectrum of 3ze 


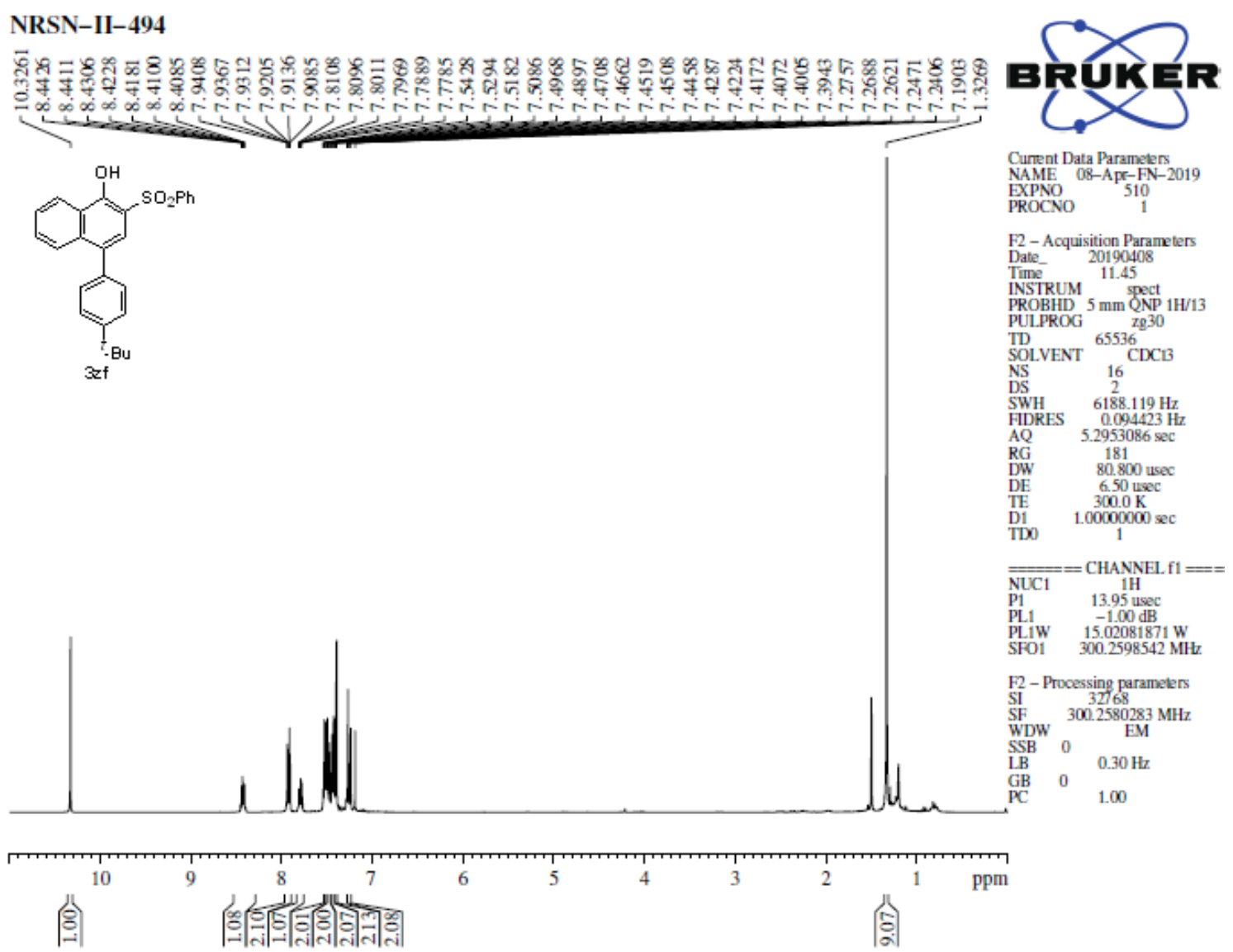

Spectrum 101: ${ }^{1} \mathrm{H}$ NMR spectrum of $\mathbf{3 z f}$

NRSN-II-494

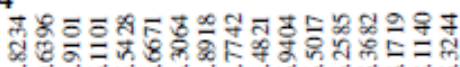
กิ

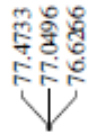

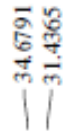

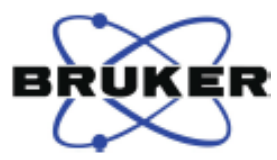

Current Data Parameters
NAME 11-April-AN-2019 EXPNO

(1)

F2- Processing parameters

$\begin{array}{lc}\text { SI } & 32768 \\ \text { SF } & 75.4999323 \mathrm{MHL}\end{array}$

WDW EM

$\underset{\mathrm{LB}}{\mathrm{SSB}} \quad 0 \quad 1.00 \mathrm{~Hz}$

$\underset{\mathrm{GB}}{\mathrm{LB}} \quad 0 \quad 1.00 \mathrm{H}$

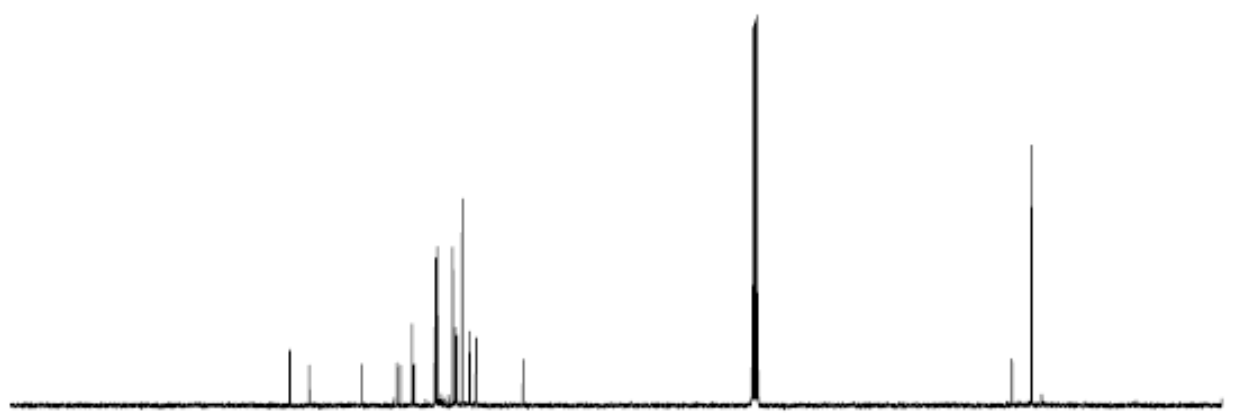

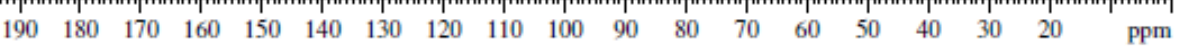

Spectrum 102: ${ }^{13} \mathrm{C}$ NMR spectrum of $\mathbf{3 z f}$ 


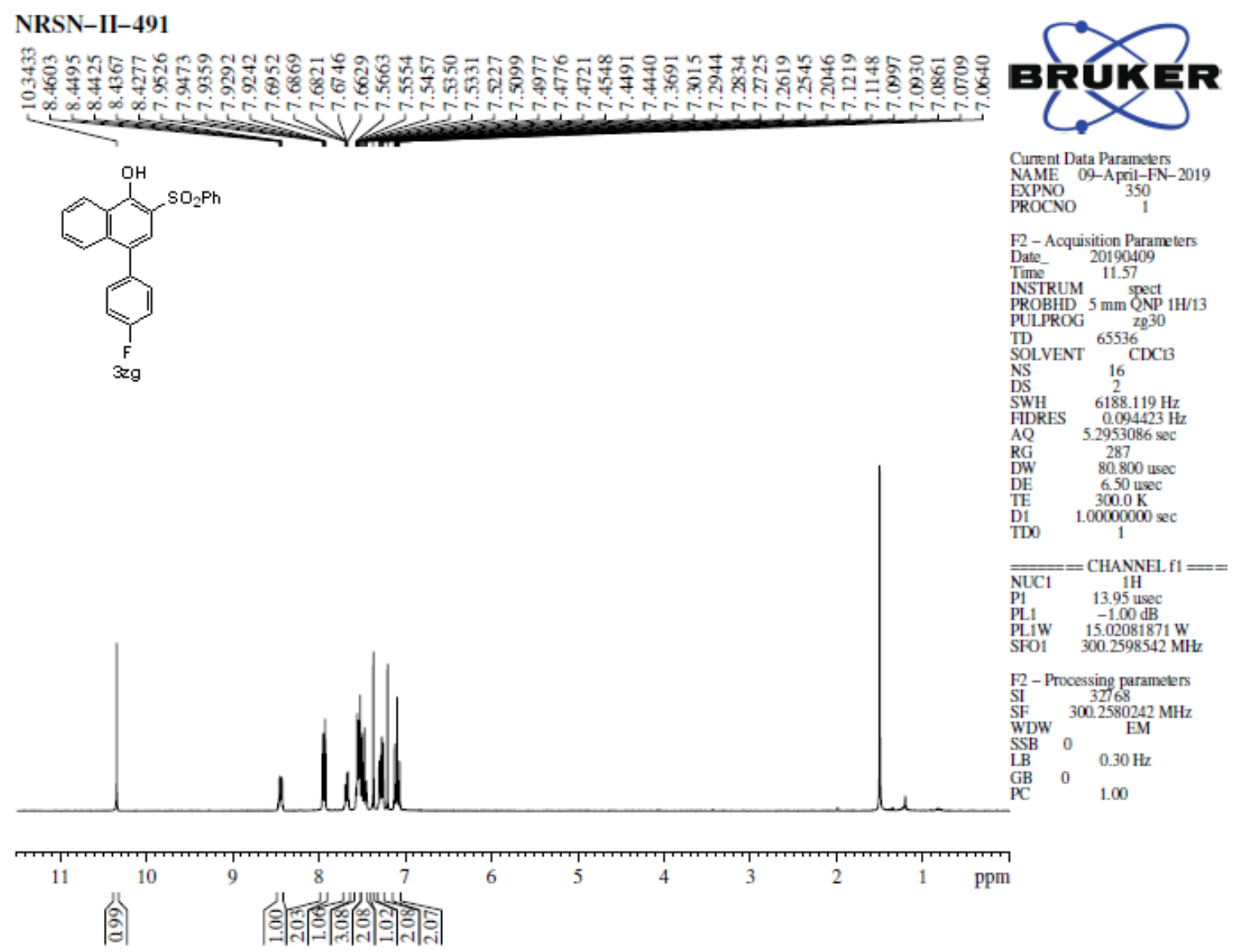

Spectrum 103: ${ }^{1} \mathrm{H}$ NMR spectrum of $\mathbf{3 z g}$

NRSN-II-491
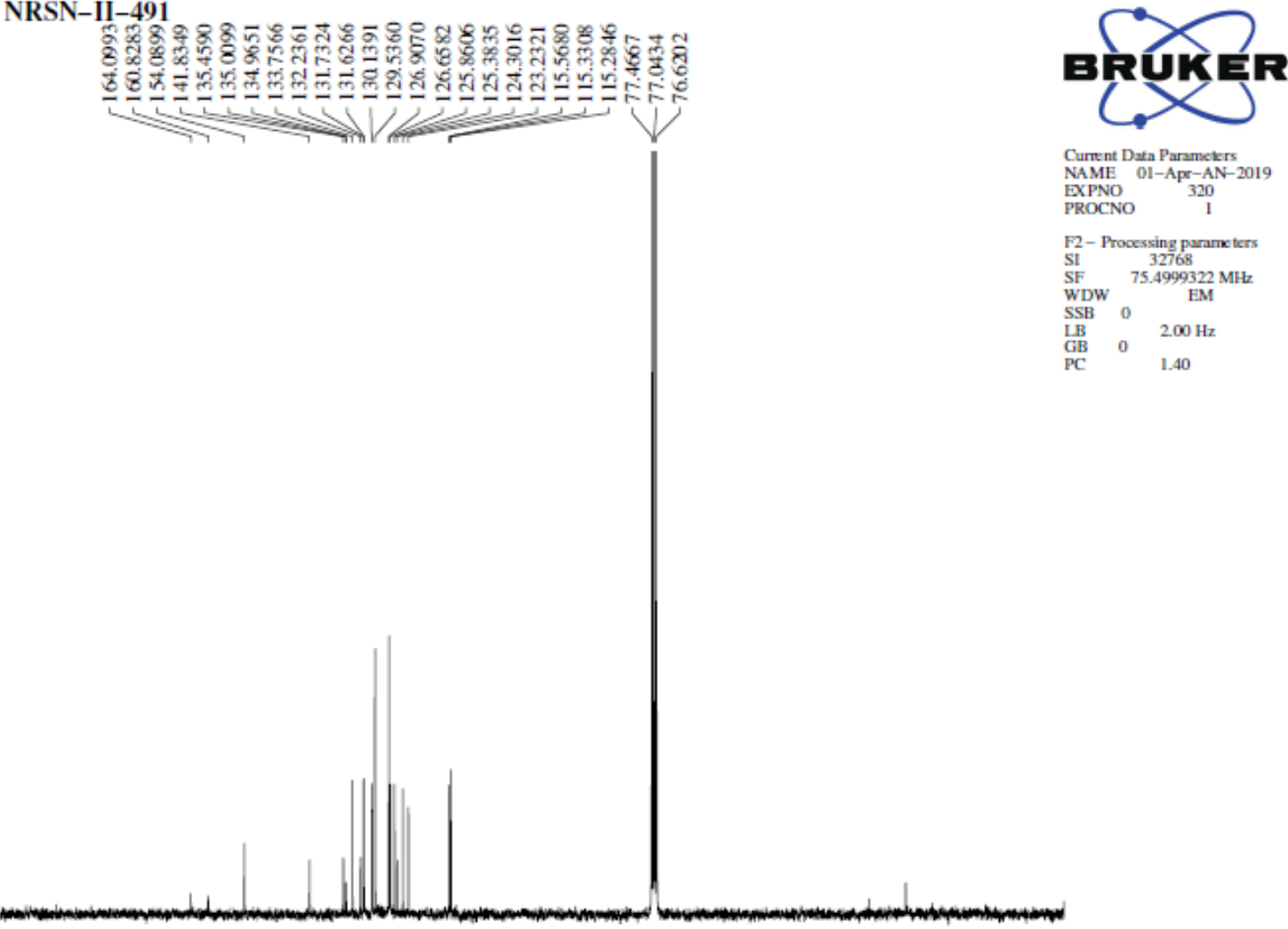

Current Data Parameters

EXPNO $\quad 320$

PROCNO

F2- Processing parame ters

$\begin{array}{ll}\text { SI } & 32768 \\ \text { SF } & 75.499932\end{array}$

WDW $\begin{aligned} 75.4999322 \\ \text { EM }\end{aligned}$

$\begin{array}{lll}\mathrm{SSB} & 0 & \\ \mathrm{LB} & 2.00 \mathrm{~Hz}\end{array}$

$\begin{array}{lll}\mathrm{LB} & 0 & 2.00 \\ \mathrm{GC} & 1.40\end{array}$

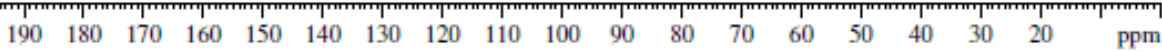

Spectrum 104: ${ }^{13} \mathrm{C}$ NMR spectrum of $3 \mathrm{zg}$ 


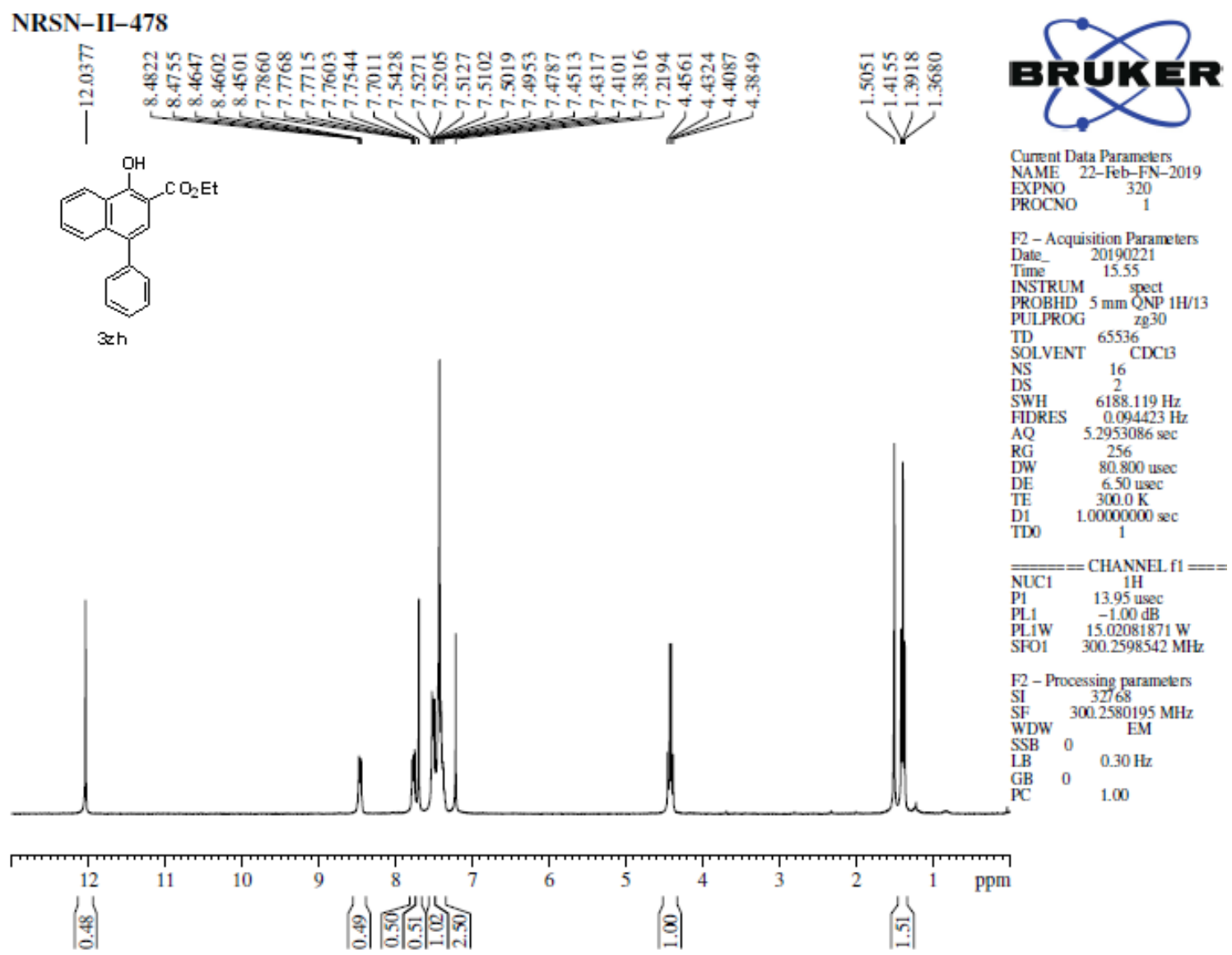

Spectrum 105: ${ }^{1} \mathrm{H}$ NMR spectrum of $\mathbf{3 z h}$
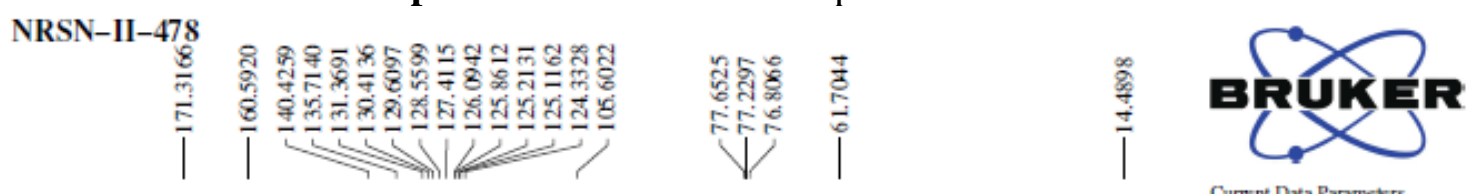

Current Data Paramekers
NAME EXPNO

PROCNO 1

F2- Processing parank ters

$\begin{array}{ll}\mathrm{SI} & 32768 \\ \mathrm{SF} & 75.4999179 \mathrm{MH}\end{array}$

WDW EM

$\begin{array}{lll}\mathrm{LB} & 1.00 \mathrm{~Hz}\end{array}$

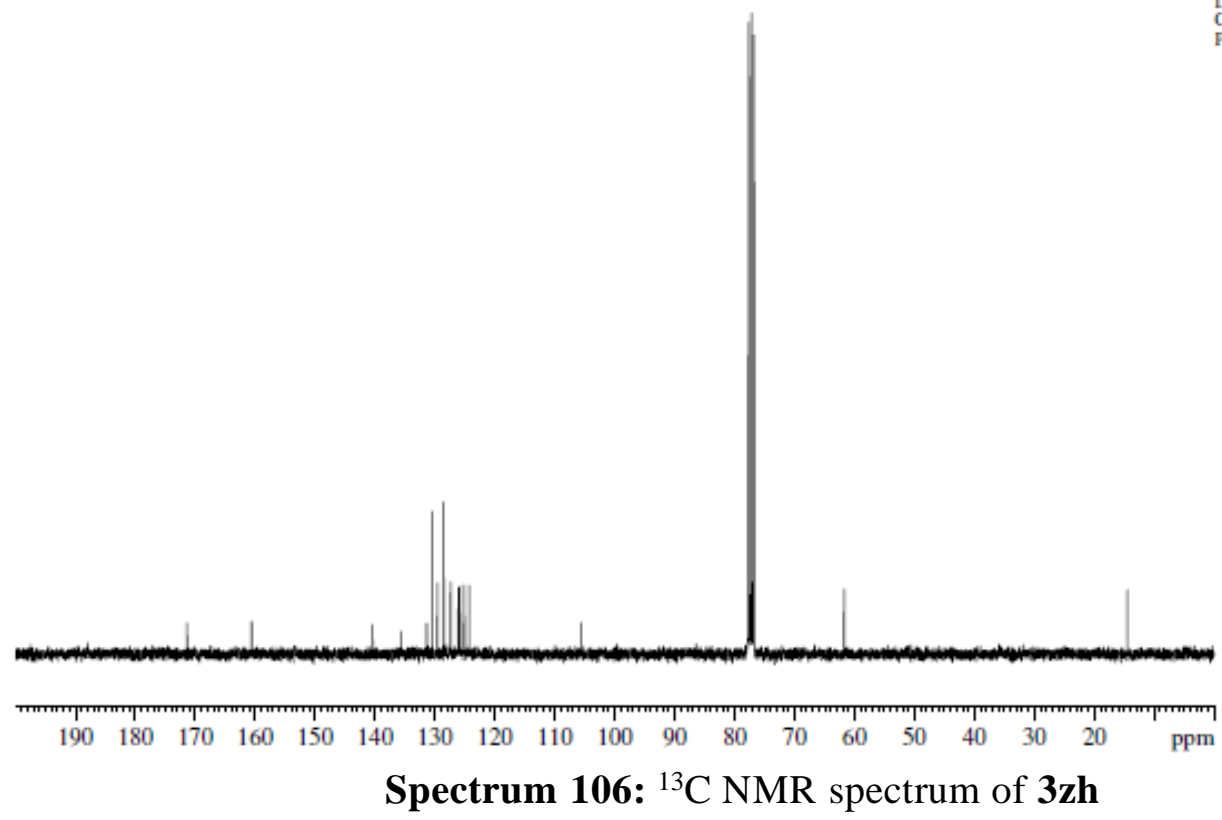




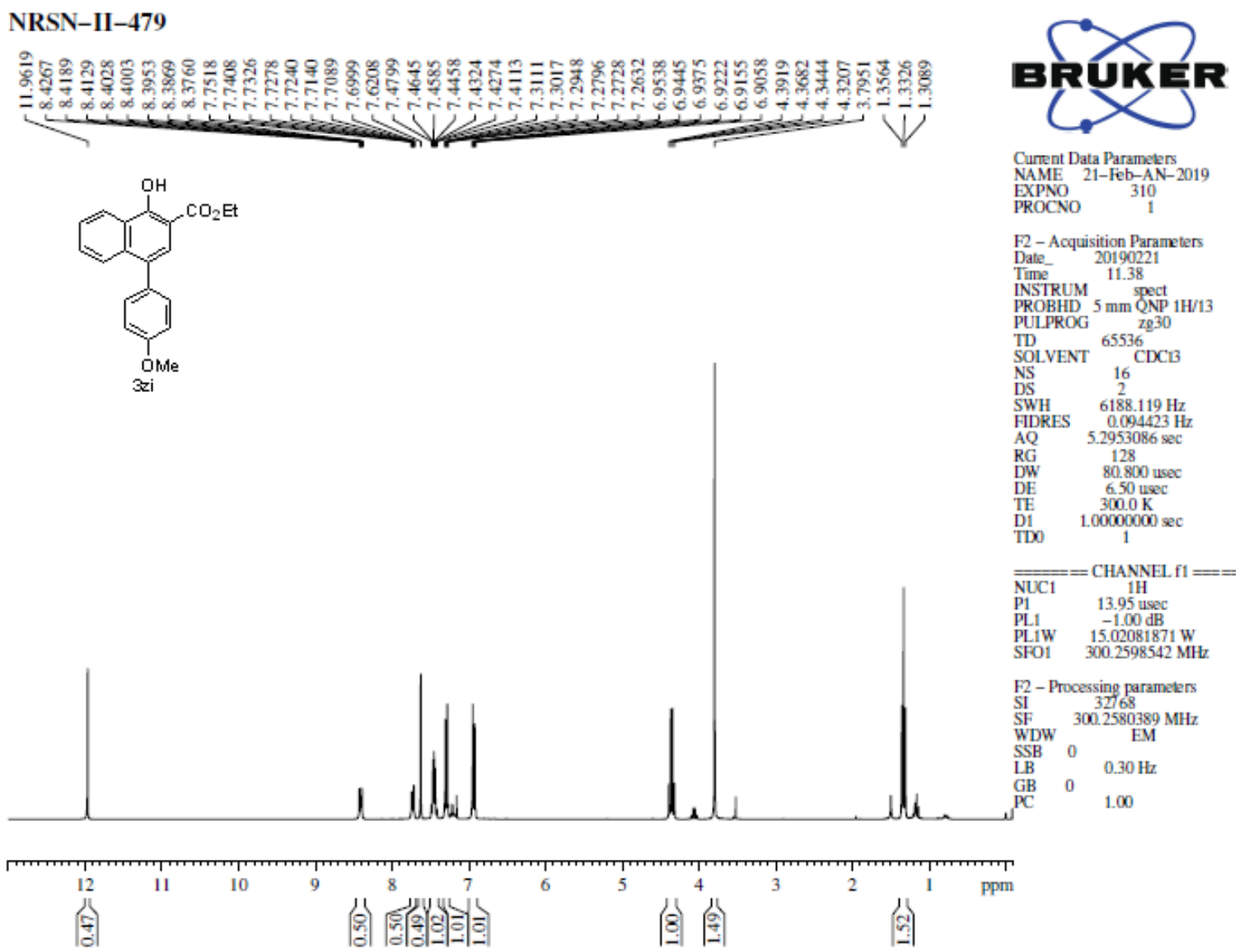

Spectrum 107: ${ }^{1} \mathrm{H}$ NMR spectrum of 3zi
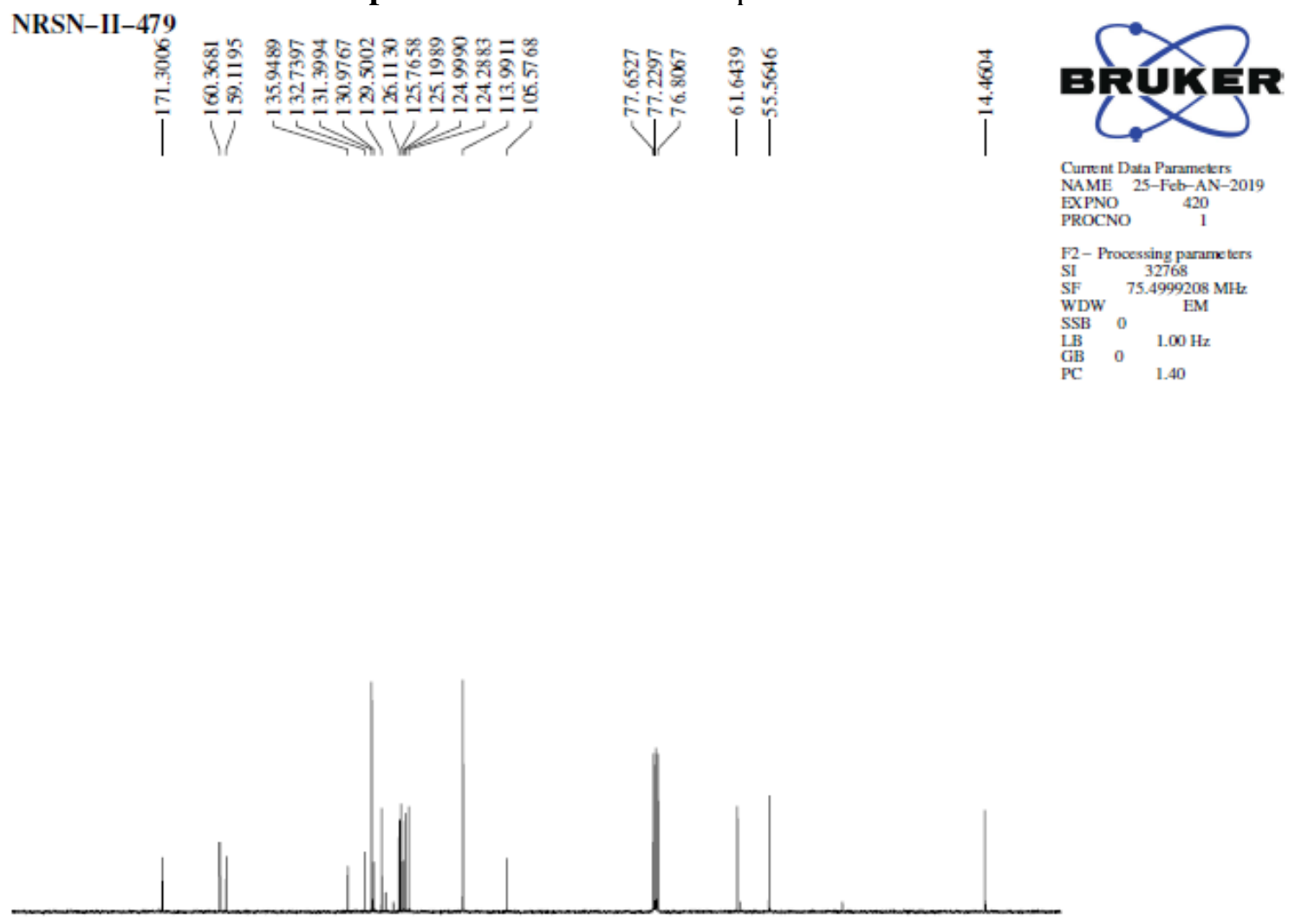

\begin{tabular}{lllllllllllllllllll}
190 & 180 & 170 & 160 & 150 & 140 & 130 & 120 & 110 & 100 & 90 & 80 & 70 & 60 & 50 & 40 & 30 & 20 & p \\
\hline
\end{tabular}

Spectrum 108: ${ }^{13} \mathrm{C}$ NMR spectrum of $\mathbf{3 z i}$ 


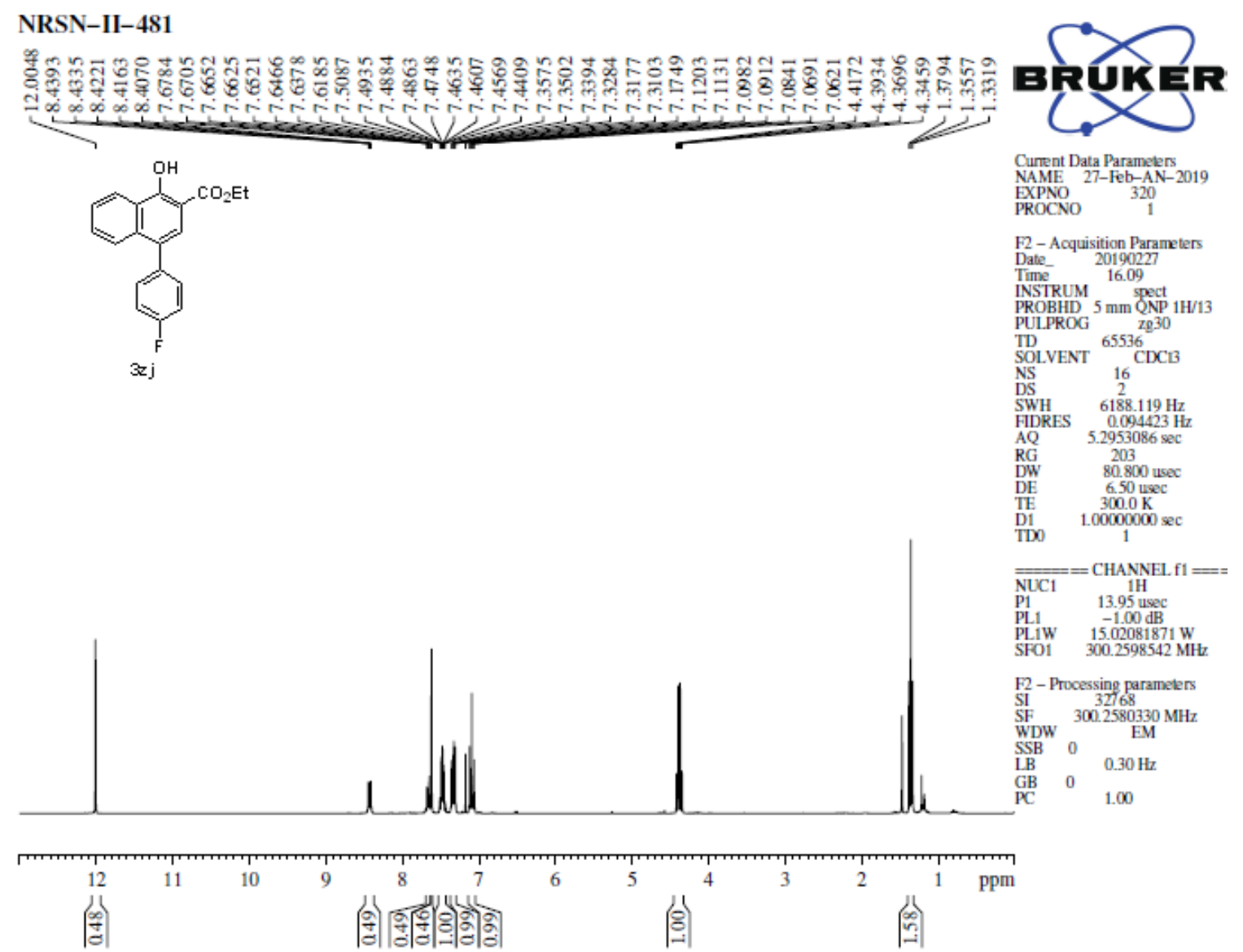

Spectrum 109: ${ }^{1} \mathrm{H}$ NMR spectrum of $\mathbf{3 z j}$

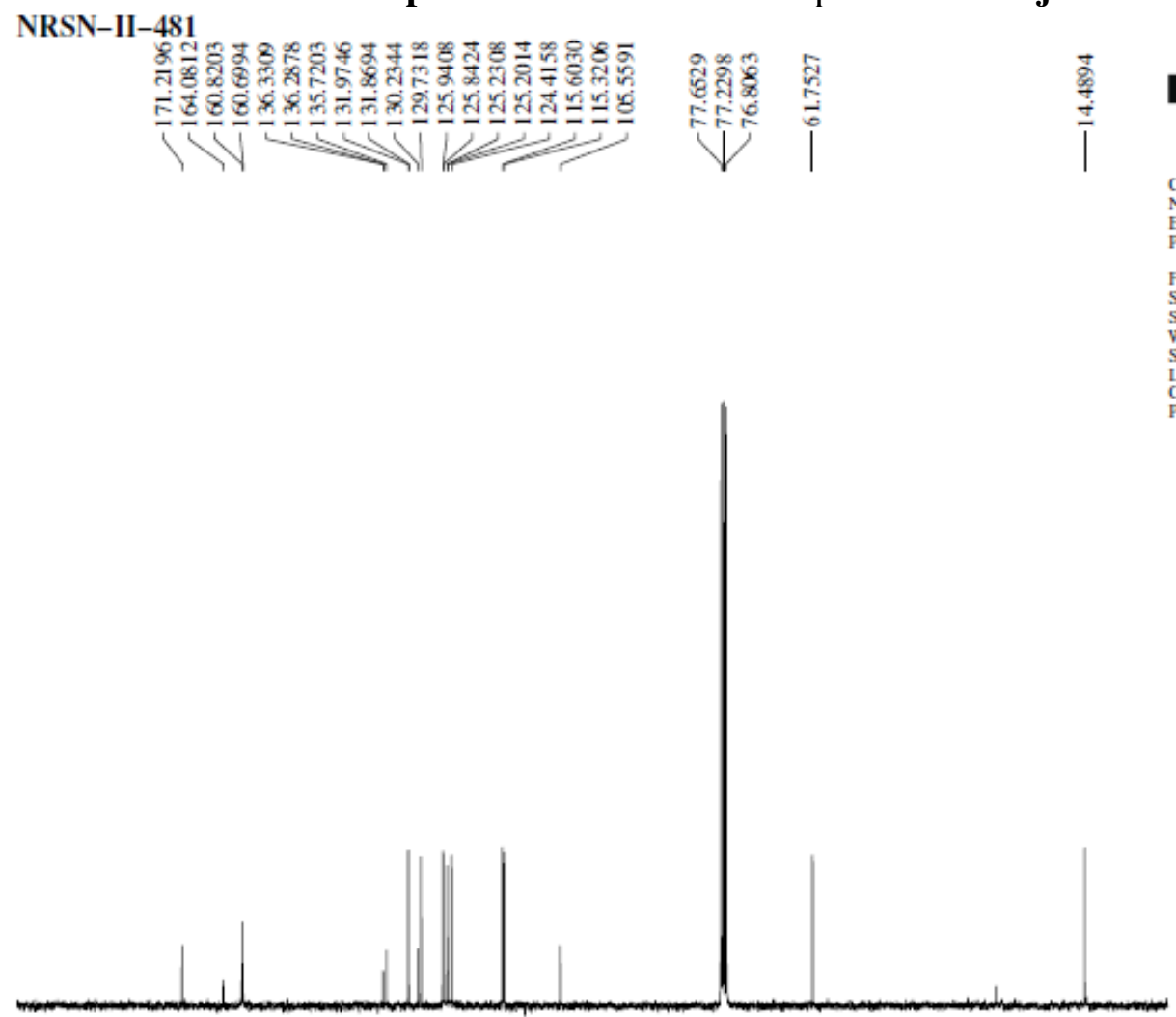

$\begin{array}{lllllllllllllllllll}190 & 180 & 170 & 160 & 150 & 140 & 130 & 120 & 110 & 100 & 90 & 80 & 70 & 60 & 50 & 40 & 30 & 20 & \mathrm{ppm}\end{array}$

Spectrum 110: ${ }^{13} \mathrm{C}$ NMR spectrum of $\mathbf{3 z j}$ 


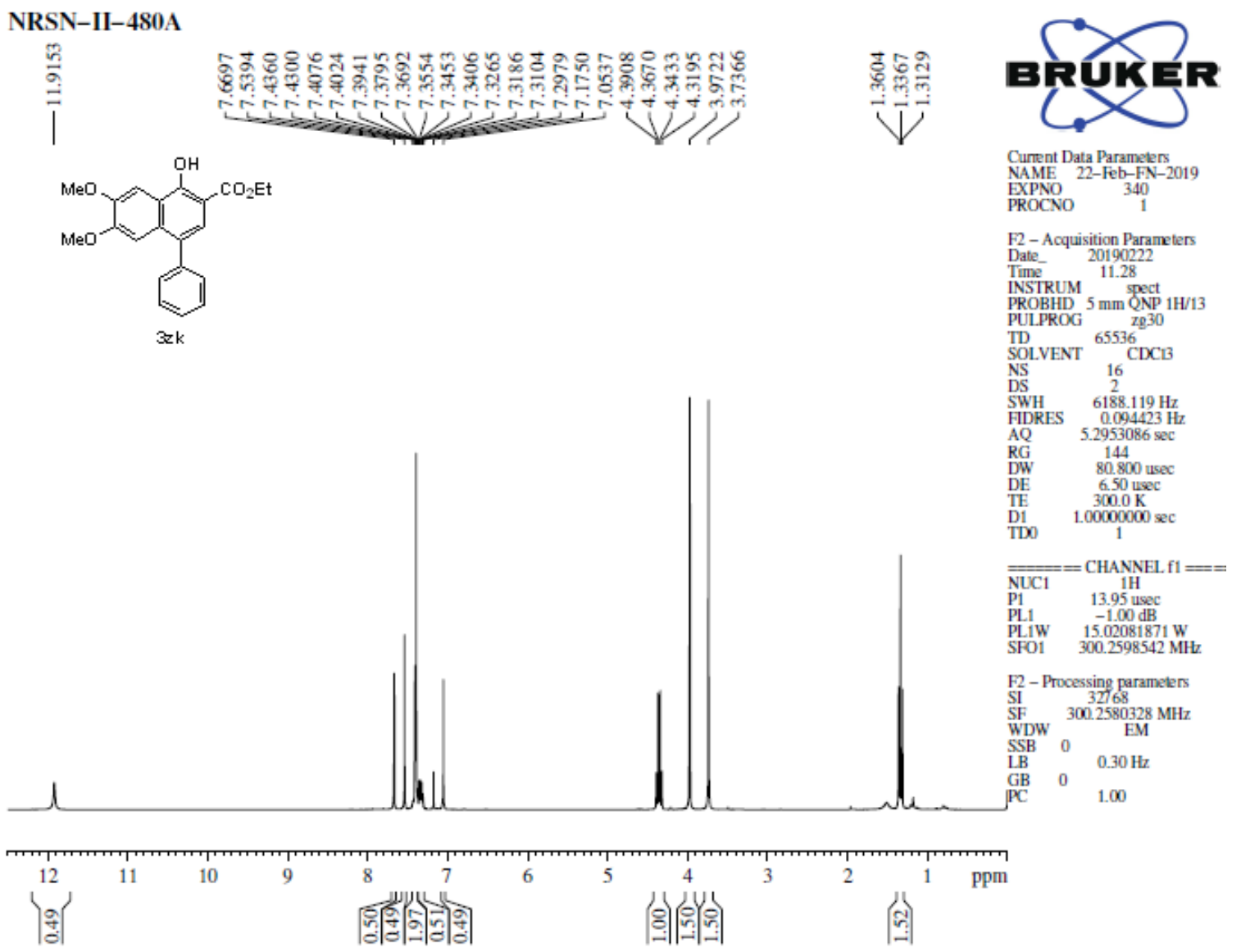

Spectrum 111: ${ }^{1} \mathrm{H}$ NMR spectrum of $\mathbf{3 z k}$
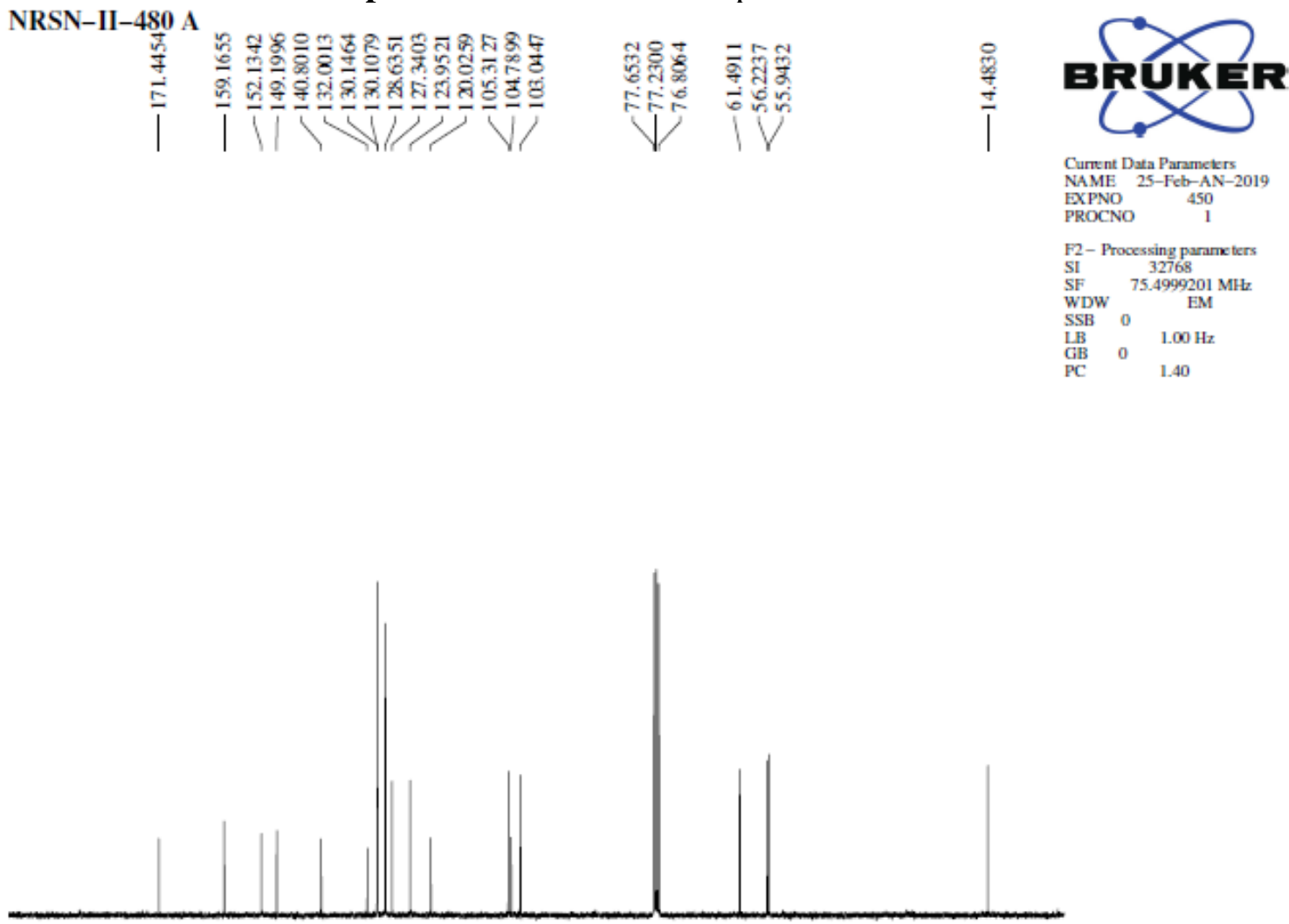

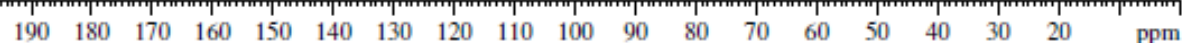

Spectrum 112: ${ }^{13} \mathrm{C}$ NMR spectrum of $3 z k$ 


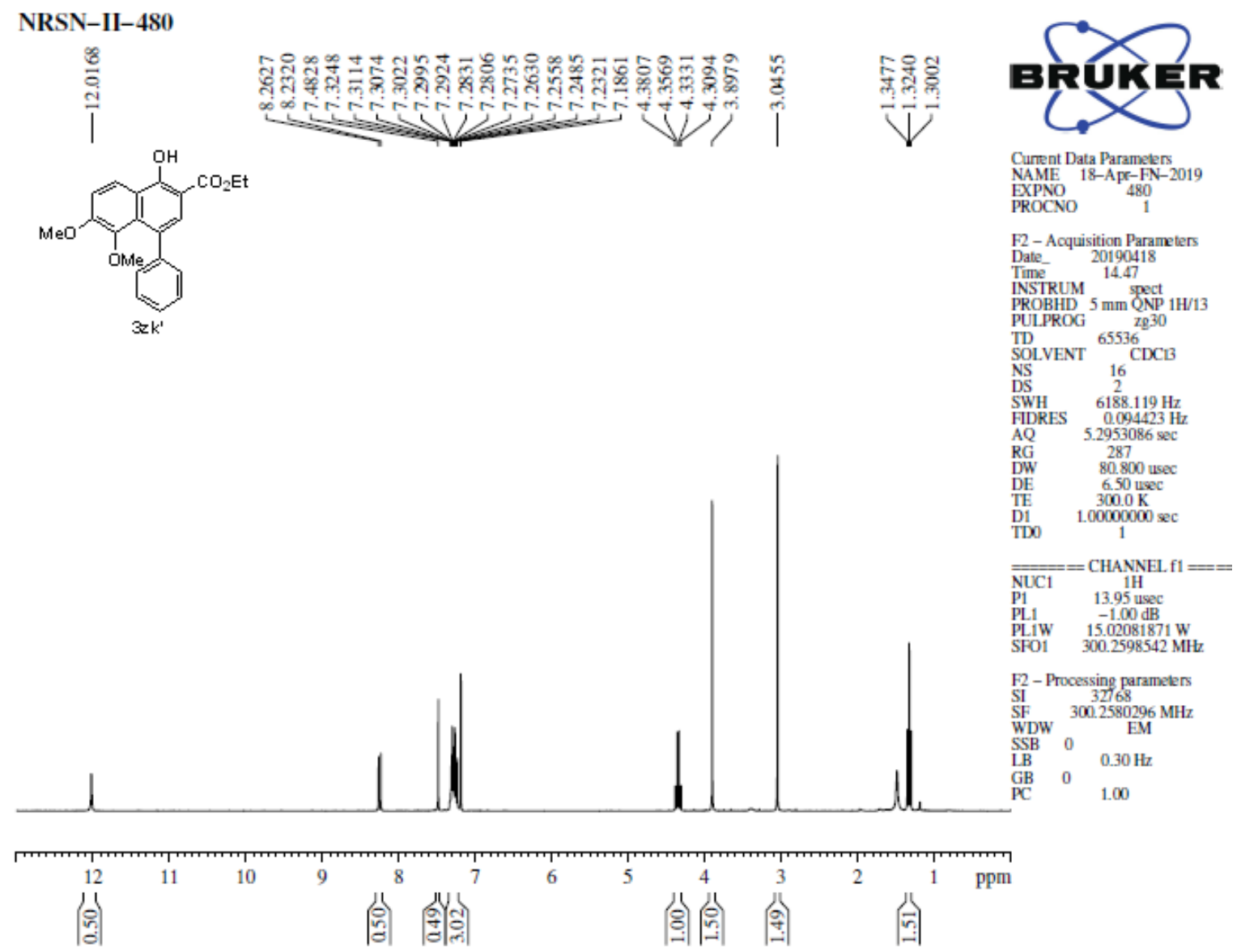

Spectrum 113: ' $\mathrm{H}$ NMR spectrum of 3zk'

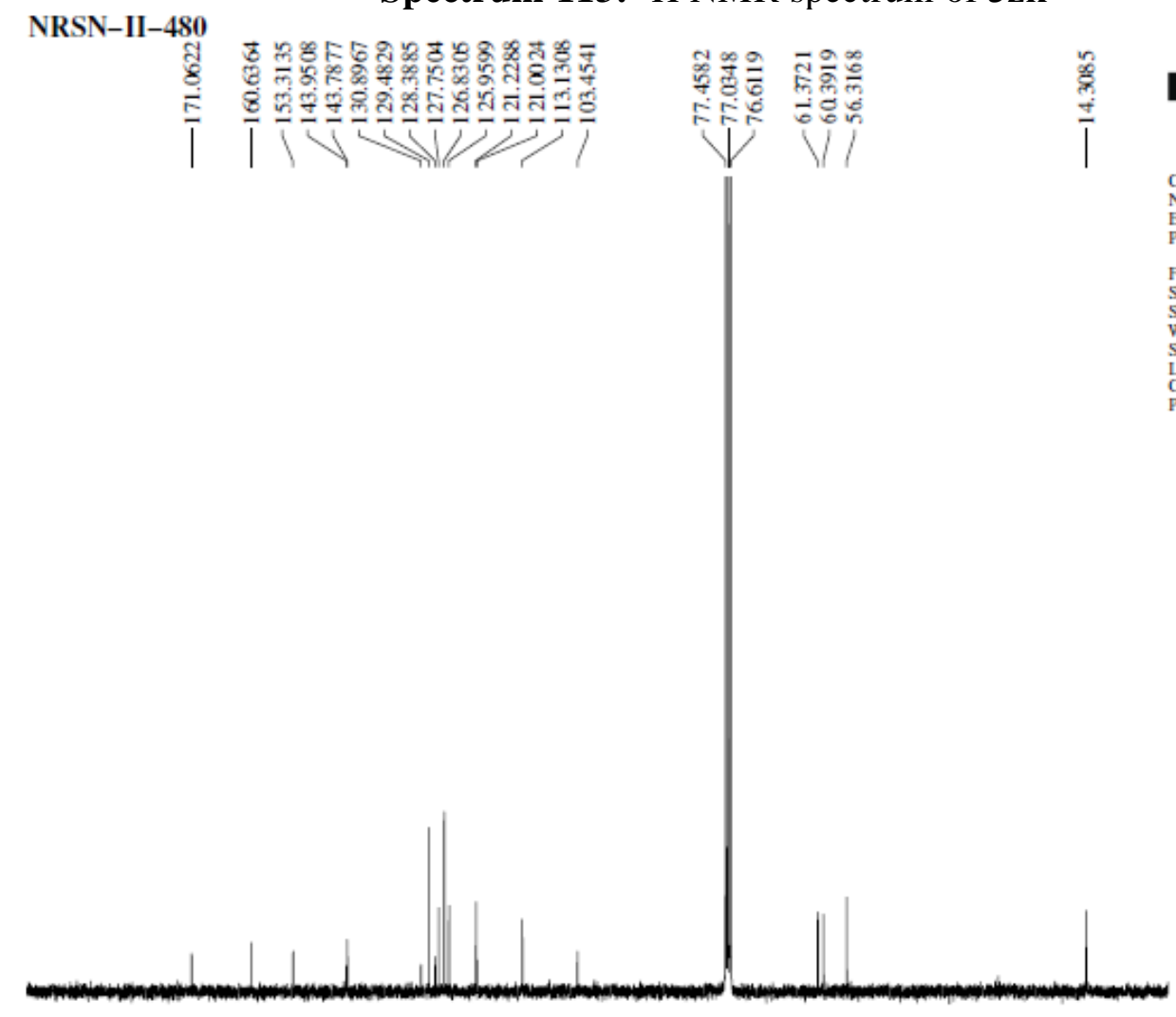

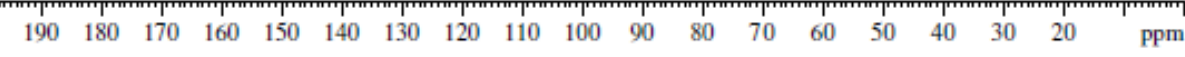
Spectrum 114: ${ }^{13} \mathrm{C}$ NMR spectrum of 3zk' 
NRSN-II-412

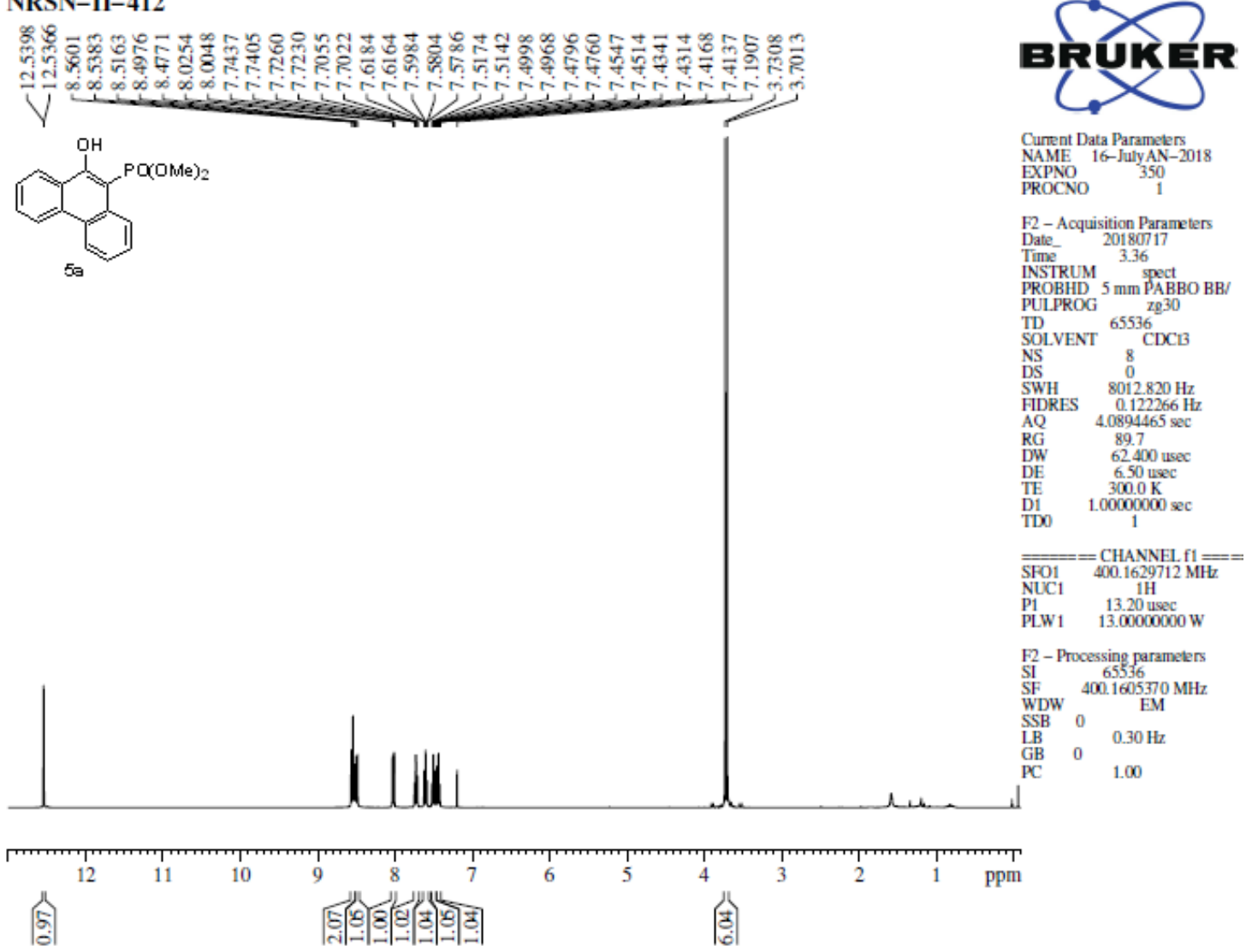

Spectrum 115: ${ }^{1} \mathrm{H}$ NMR spectrum of $\mathbf{5 a}$
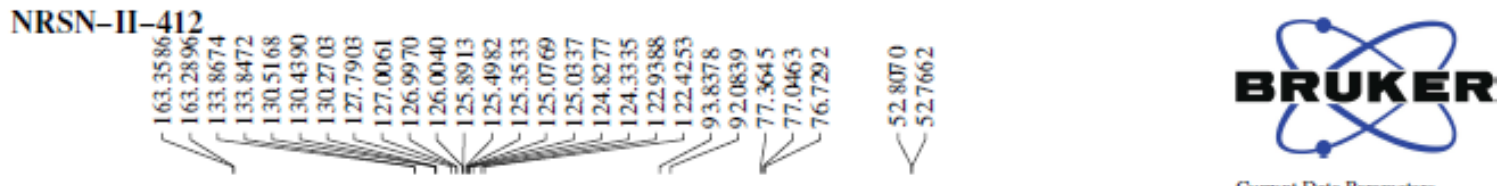

Current Data Paramekers

NAME 18-July-AN-2018

EXPNO

PROCNO 1

F2- Acquisition Parameters

$\begin{array}{lc}\text { Date_- } & 20180718 \\ \text { Time } & 15.15\end{array}$

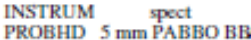

PROBHD $5 \mathrm{~mm}$ PABBO
PULPROG $\quad 2 \mathrm{gpg} 30$

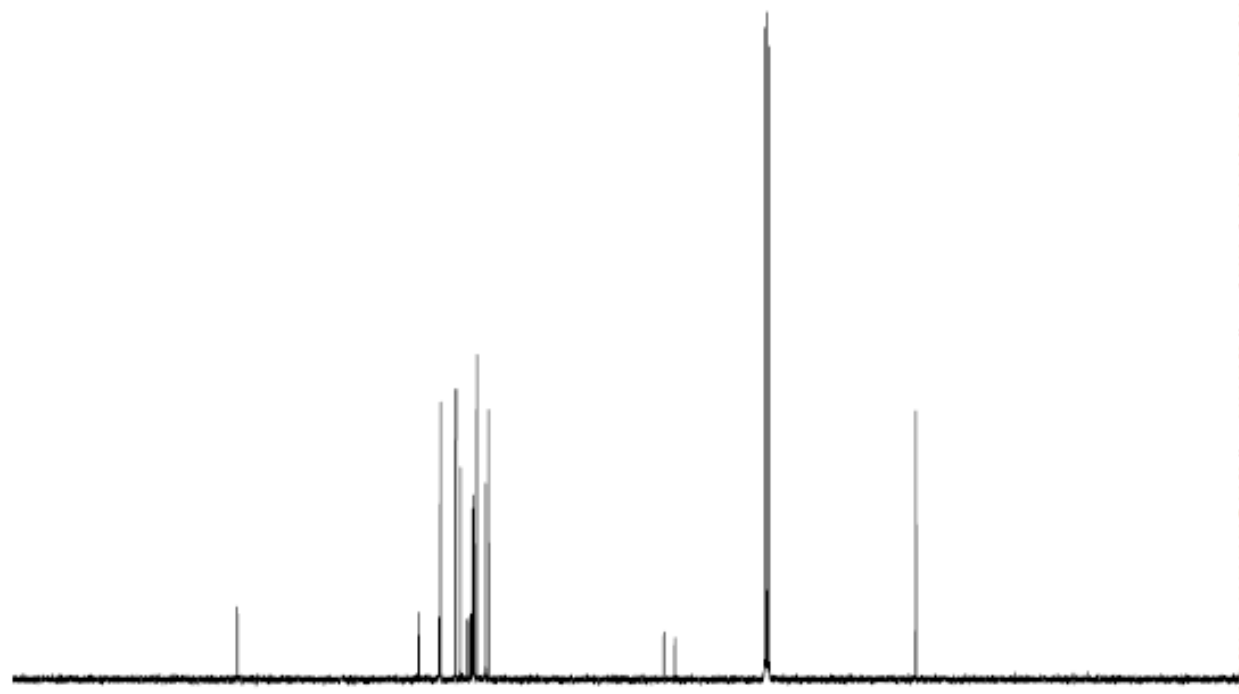

TDLPROG $6553 \mathrm{z}^{2 \mathrm{gg} 30}$

SOLVENT CDC13

$\begin{array}{lc}\text { NS } & 1024 \\ \text { DS } & 4\end{array}$

SWH $\quad 24038.461 \mathrm{~Hz}$

FDRES $\begin{array}{ll}0.366798 \mathrm{~Hz} \\ \mathrm{AQ}\end{array}$

$\begin{array}{ll}\text { AQ } & 1.3631488 \\ \text { RG } & 201.48\end{array}$

$\begin{array}{ll}\text { RG } & 201.48 \\ \text { DW } & 20.800 \text { useo }\end{array}$

6.50 use

$300.0 \mathrm{~K}$

$200000000 \mathrm{sec}$
$0.030000000 \mathrm{sec}$

$\begin{array}{rr}\text { DI1 } & 0.03000000 \\ & 1\end{array}$

CHANNEL $\mathrm{fl}=$

SFOl $1006704993 \mathrm{MH}$

NUCl $\quad 13 \mathrm{C}$

$\begin{array}{ll}\text { Pl } & 9.90 \text { uxe } \\ \text { PLW1 53.00000000 W }\end{array}$

SFO2 $400.1621006 \mathrm{MHz}$

SFO2 $400.1621006 \mathrm{M}$

CPDPRG[2 1 waltz16

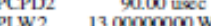

$0.27963999 \mathrm{~W}$

PLW $13 \quad 0.22651000 \mathrm{~W}$

72 - Processing parame ter: $\begin{array}{ll}\mathrm{SI} & 32768 \\ \mathrm{SF} & 100.6204380 \mathrm{MHz}\end{array}$

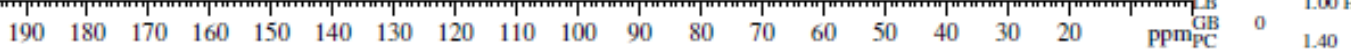

Spectrum 116: ${ }^{13} \mathrm{C}$ NMR spectrum of $\mathbf{5 a}$ 


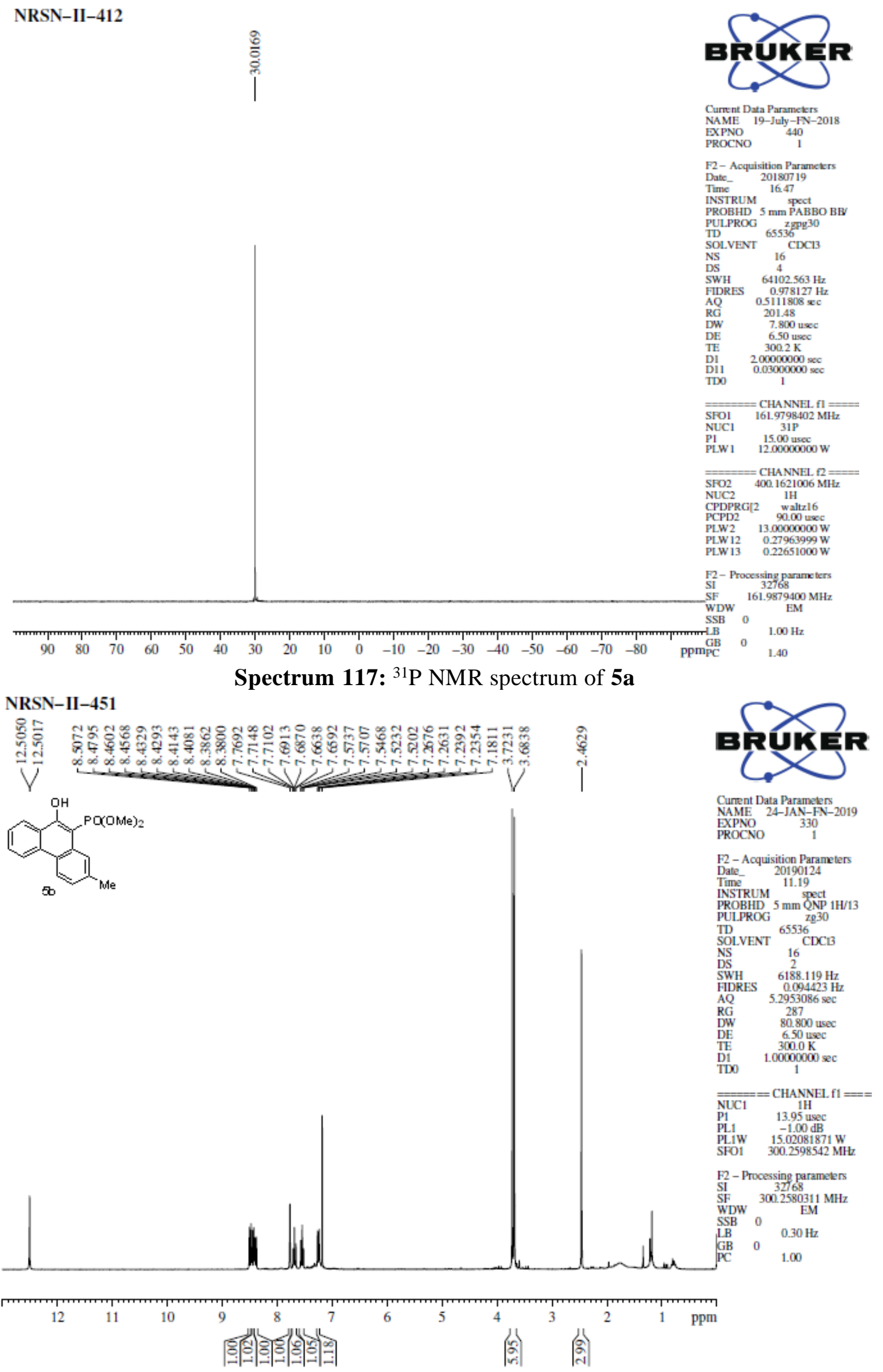

Spectrum 118: ${ }^{1} \mathrm{H}$ NMR spectrum of $\mathbf{5 b}$ 


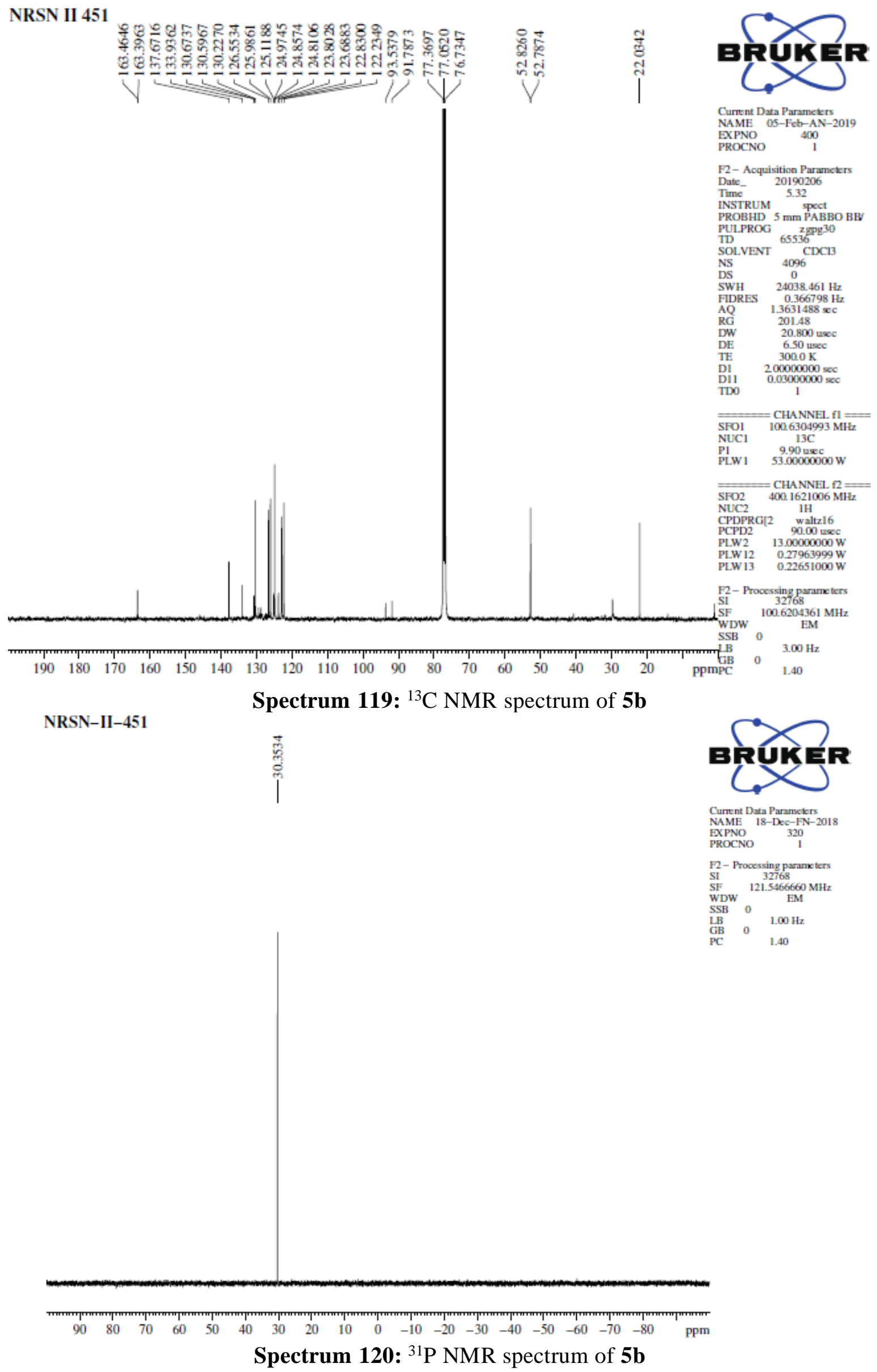




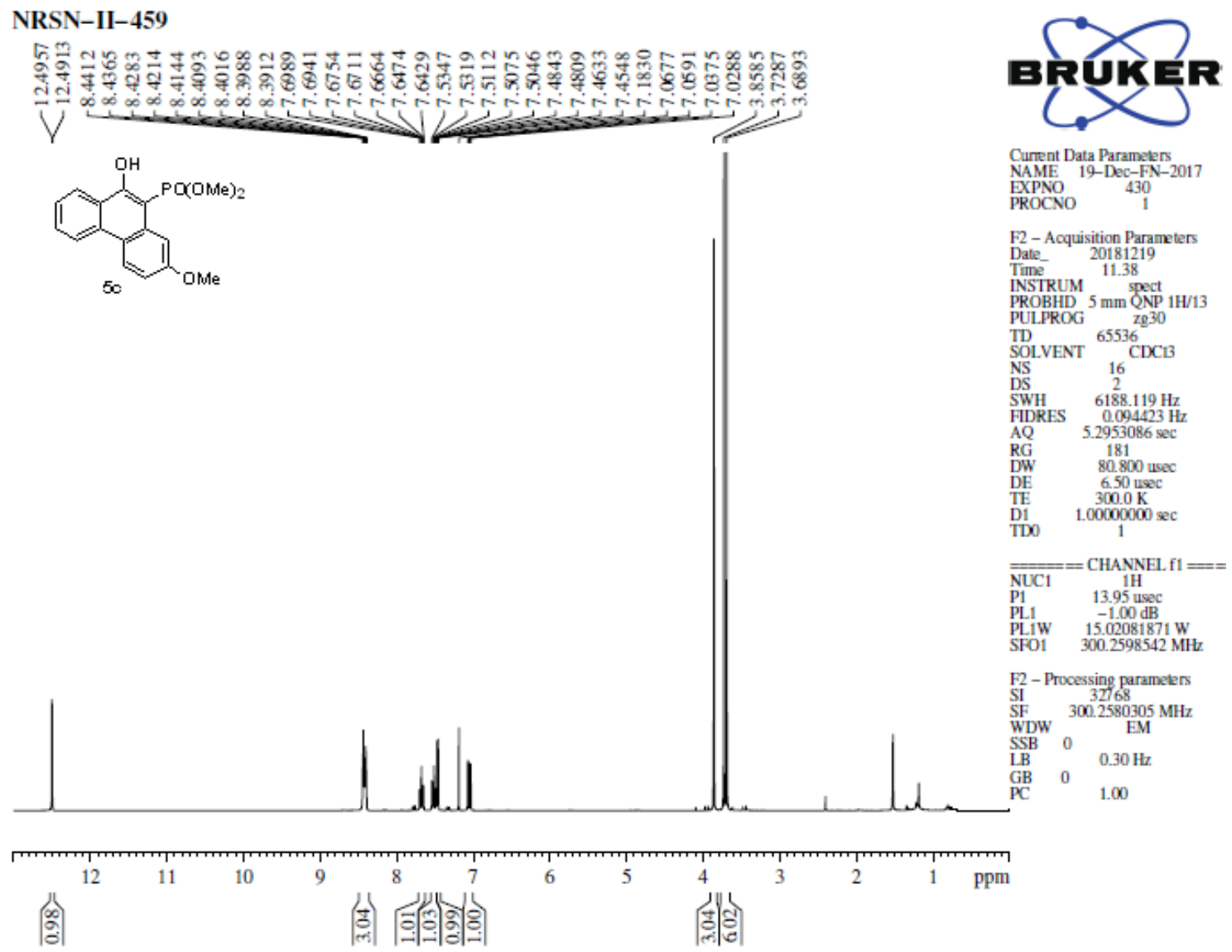

Spectrum 121: ${ }^{1} \mathrm{H}$ NMR spectrum of $5 \mathrm{c}$
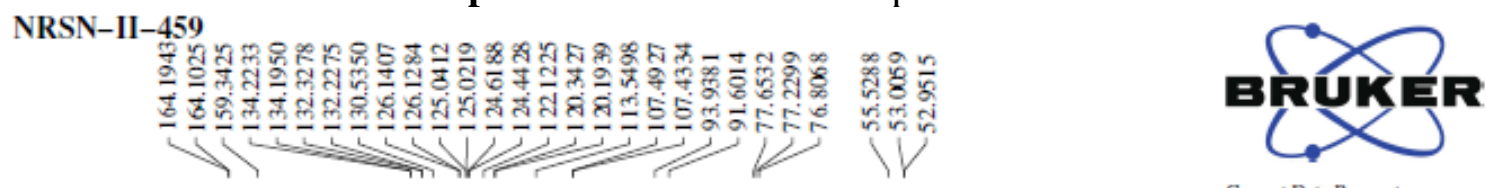

Curnent Data Parameters

$\begin{array}{lc}\text { NAME } & 20-D e c-A N-201 \\ \text { EXPNO } & 350\end{array}$

EXPNO
PROCNO

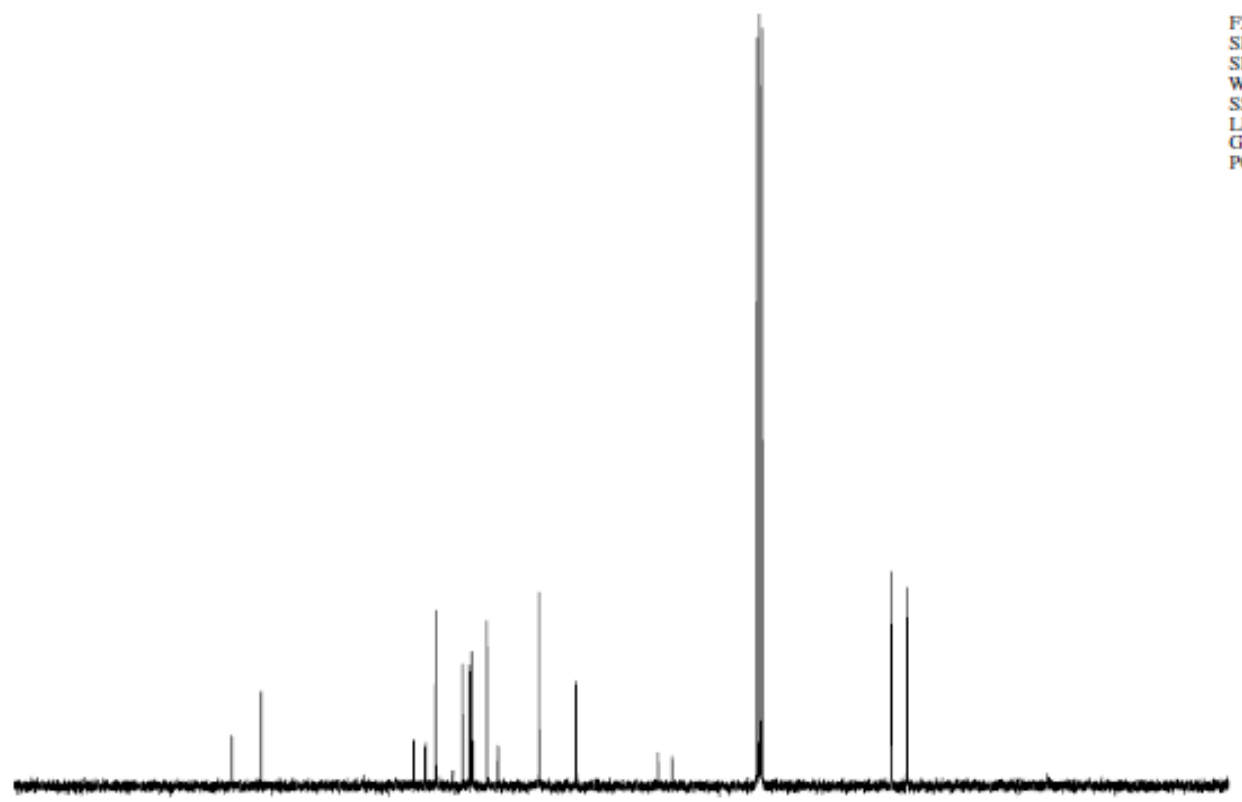

F2- Processing parame ter

$\begin{array}{ll}\text { SI } & 32768 \\ \mathrm{SF} & 75.4999189 \mathrm{MH}\end{array}$

EM

$\begin{array}{lll}\mathrm{SSB} & 0 & \\ \mathrm{LB} & & \end{array}$

$\underset{\mathrm{GB}}{\mathrm{GB}} \quad 0 \quad 1.00$

$\begin{array}{lllllllllllllllllll}190 & 180 & 170 & 160 & 150 & 140 & 130 & 120 & 110 & 100 & 90 & 80 & 70 & 60 & 50 & 40 & 30 & 20 & \mathrm{ppm}\end{array}$

Spectrum 122: ${ }^{13} \mathrm{C}$ NMR spectrum of $5 \mathrm{c}$ 

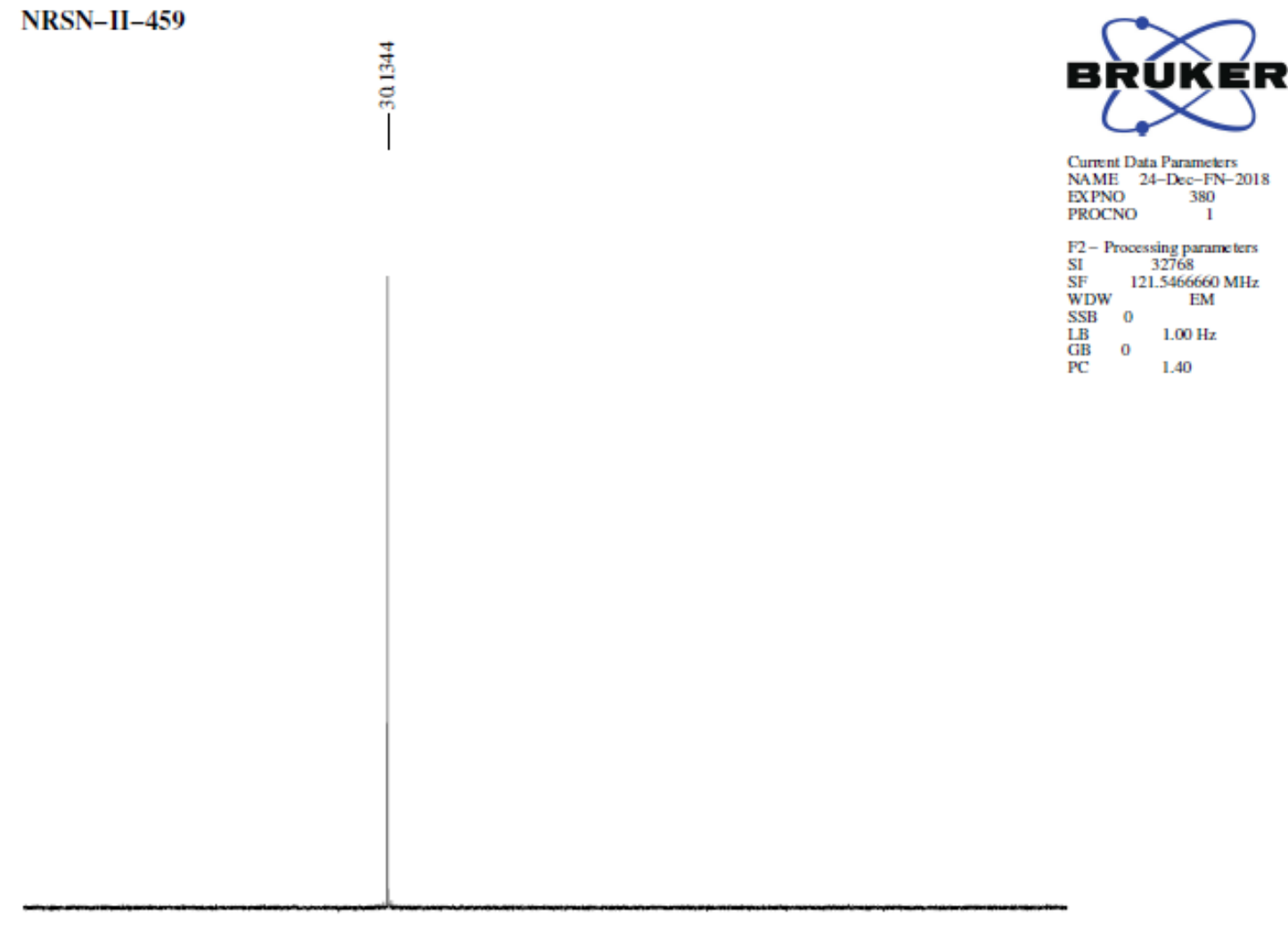

Current Data Parameters

NAME 24-Dec-FN-2018

PRPNO

F2- Processing parancters

$\begin{array}{ll}\mathrm{SI} & 32768 \\ \mathrm{SF} & 121.5466660 \mathrm{MHz}\end{array}$

WDW EM

SSB $0 \quad 1.00 \mathrm{~Hz}$

$\begin{array}{lll}\mathrm{LB} & 0 & 1.00 \mathrm{H} \\ \mathrm{GC} & 0 & 1.40\end{array}$

Spectrum 123: ${ }^{31} \mathrm{P}$ NMR spectrum of $5 \mathrm{c}$

NRSN-II-419
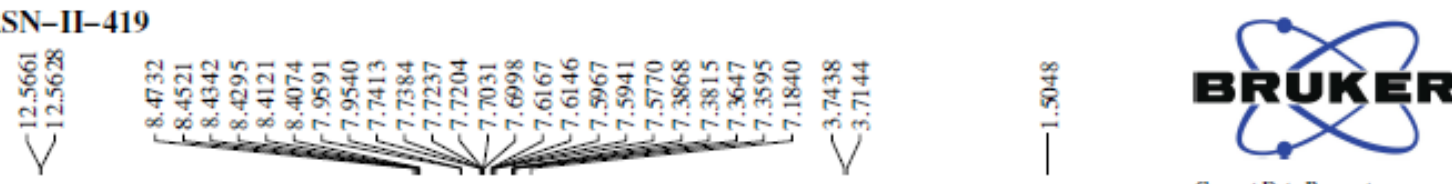<smiles>CC(=O)Oc1c(O)c2ccccc2c2cc(Cl)ccc12</smiles>

Cuntent Data Parameters

NAME

PROCNO

F2 - Acquisition Parameters

Date_ 20180723

INSTRUM 13.58

INSTRUM spect

PULPROG ze 30

TD

SOLVENT ${ }_{8} \mathrm{CDCB}$

$\begin{array}{ll}\text { DS } & 0 \\ \text { SWH } & 8012.820 \mathrm{~Hz}\end{array}$

$\begin{array}{ll}\text { SWH } & 8012.820 \mathrm{~Hz} \\ \text { FIDRES } & 0.122266 \mathrm{~Hz}\end{array}$

FIDRES

$\begin{array}{ll}\text { AQ } & 4.0894465 \\ \text { RG } & 159.22\end{array}$

DW $\quad 62.400$ usec

6.50 use
DE $\quad 300.0 \mathrm{~K}$

TE $\quad 300.0 \mathrm{~K}$

$\overline{\text { FO1 }}=\underset{400.1629712 \mathrm{MHz}}{\text { CHANNEL }}$

NUC1 $1 \mathrm{H}$

$\begin{array}{ll}\text { P1 } & 13.20 \text { usec } \\ \text { PLW1 } & 13.00000000 \mathrm{~W}\end{array}$

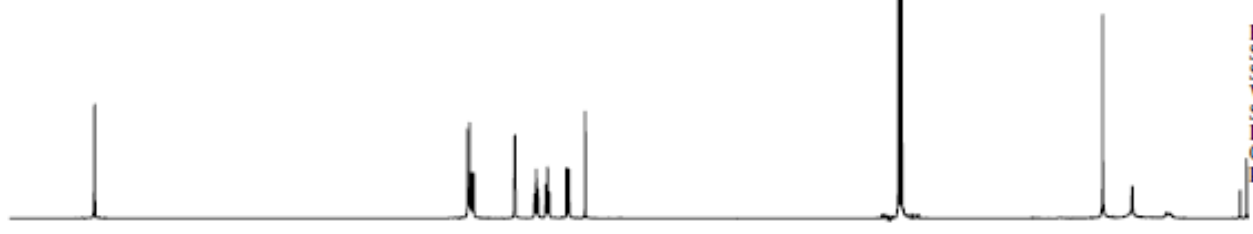

F2 - Processing parameters
SI

SI $\quad \begin{array}{ll}65336 \\ \text { SF } & 400.1605401 \mathrm{MHz}\end{array}$

WDW $400.1605401 \mathrm{MHz}$

WDW
SSB
LB

B $\quad 0.30 \mathrm{~Hz}$

$\begin{array}{lll}\mathrm{GB} & 0 & 100\end{array}$

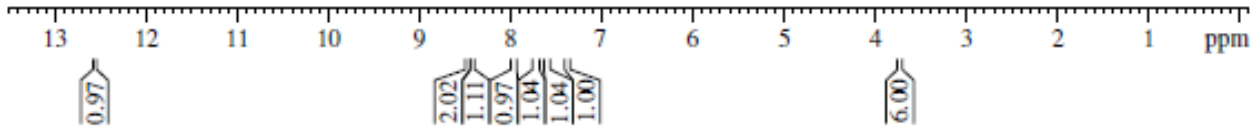

Spectrum 124: ${ }^{1} \mathrm{H}$ NMR spectrum of $5 \mathrm{~d}$ 


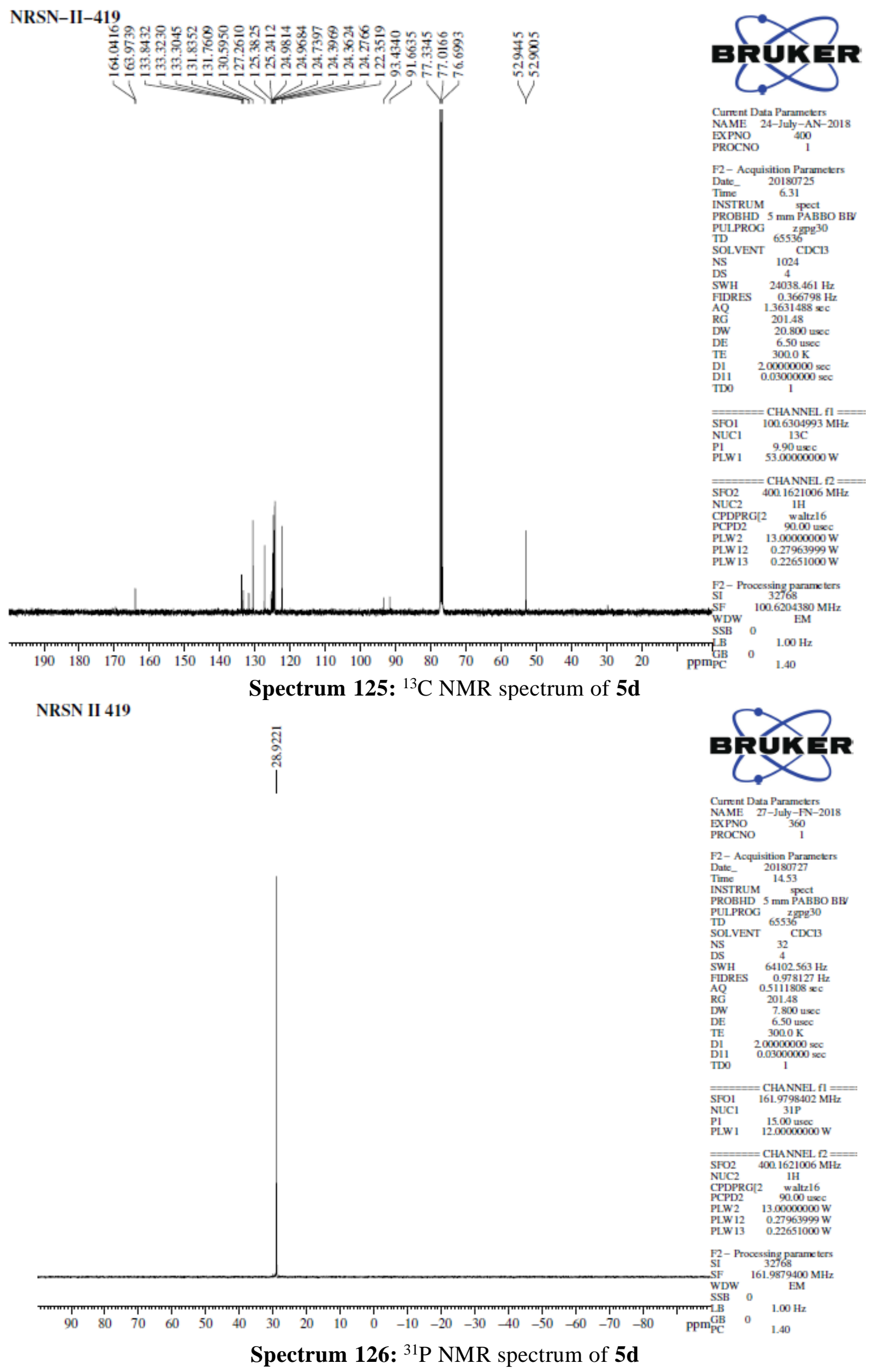


NRSN-II-440

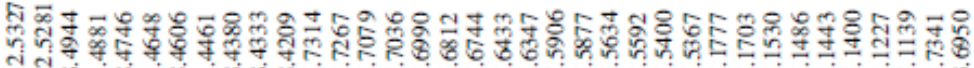

I<smiles>COC(=O)c1c(O)c2ccccc2c2ccc(F)cc12</smiles>
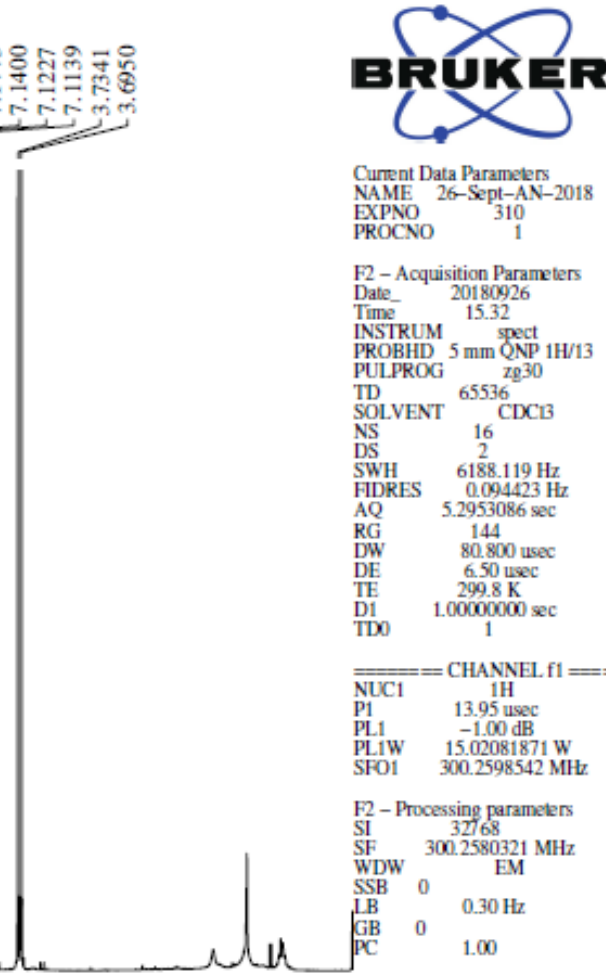

Cuntent Data Parameters 2018

EXPNO

PROCNO

F2 - Acquisition Parameters

Time $\quad 15.32$

INSTRUM spect

PROBHD $5 \mathrm{~mm} \mathrm{QNP} \mathrm{1H/13}$

$\begin{array}{ll}\text { PULPROG } & \text { zg.30 } \\ \text { TD } & 65536\end{array}$

${ }_{\text {SOLVENT }}^{65536} \mathrm{CDCl}^{2}$

NS 16

$\begin{array}{ll}\text { DS } & 2 \\ \text { SWH } & 6188.119 \mathrm{~Hz}\end{array}$

FIDRES $\quad 0.094423 \mathrm{H}$

AQ $\quad 5.2953086 \mathrm{sec}$

$\begin{array}{ll}\text { RG } & 144 \\ \text { DW } & 80.800 \text { use }\end{array}$ 80.800 usec
6.50 usec 6.50 usec
$299.8 \mathrm{~K}$
$00000000 \mathrm{sec}$

D1 $1.00000000 \mathrm{sec}$

$\overline{\overline{N U C 1}}=$ CHANNEL $\mathrm{f} 1=$ $\begin{array}{ll}\text { NUC1 } & 1 \mathrm{H} \\ \text { P1 } & 13.95 \text { usec }\end{array}$

PLIW $\quad-15.0008 \mathrm{~dB}$

SFO1 $300.2598542 \mathrm{MHz}$

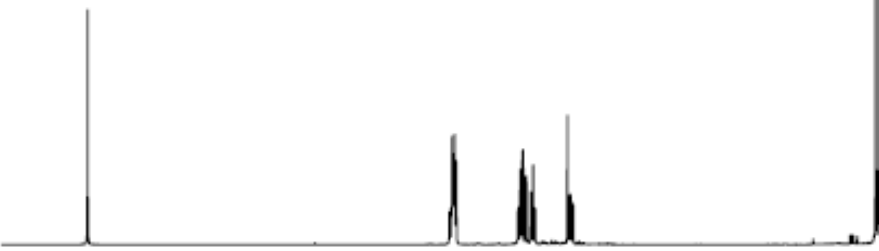

F2-Processing parameters SI $\quad 300.2580321 \mathrm{MHz}$ WDW $\begin{array}{ll}300.2580321 \\ \text { EM }\end{array}$ SSB 0 LB $0.30 \mathrm{~Hz}$ $\mathrm{PC}$

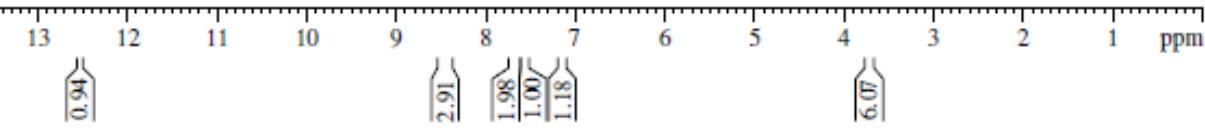

Spectrum 127: ${ }^{1} \mathrm{H}$ NMR spectrum of $5 \mathbf{e}$

NRSN II 440
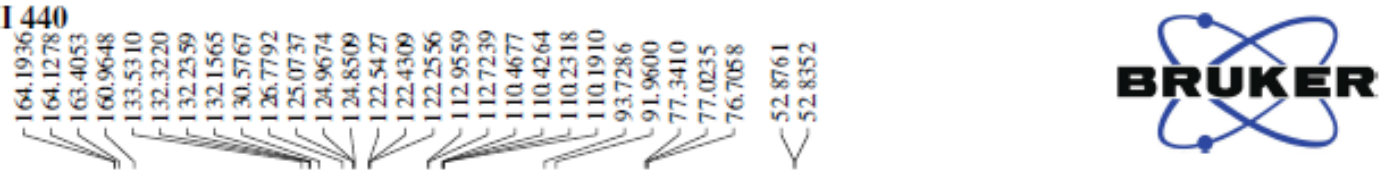

Curnent Dita Parameters

NAME 23-Jan-AN-201 PROCNO

F2- Acquisition Parameters

Date_ 20190123

Time 21.35

INSTRUM spect

PROBHD $5 \mathrm{~mm}$ PABBO B

PULPROG $2 \mathrm{~m}^{2} \mathrm{gpg} 30$

SOLVENT CDC13

NS 4096

DS $\quad 0006$

SWH $\quad 24038.461 \mathrm{~Hz}$

FIDRES $\begin{aligned} & 0.366798 \mathrm{~Hz} \\ & \mathrm{~A}\end{aligned}$

$\begin{array}{ll}\text { AQ } & 1.3631488 \\ \text { RG } & 201.48\end{array}$

DW $\quad 20.800$ used

DE $\quad 3.50$ usec

DI $2000000000 \mathrm{sec}$

Dil $0.03000000 \times$

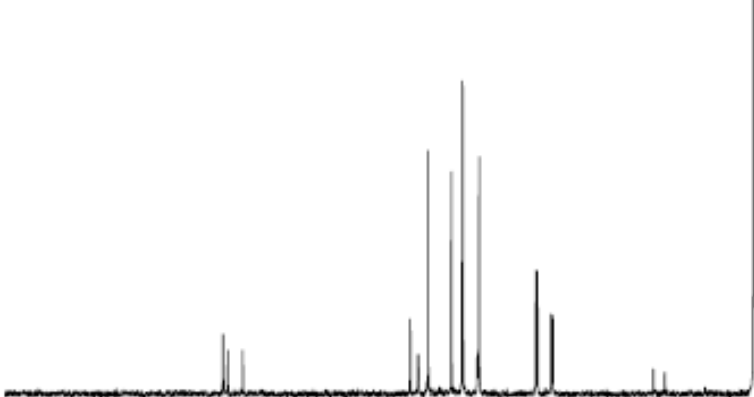

CHANNEL $\mathrm{fl}=$

SFOl $100.6304993 \mathrm{MP}$

NuCl $13 \mathrm{C}$

$\begin{array}{ll}\text { P1 } & 9.90 \text { uxe } \\ \text { PLW1 } & 53.00000000 \mathrm{~W}\end{array}$

CHAN $\quad$ CHNEL $1621006 \mathrm{MHz}$

NUC2 $\quad 100.162100$

CPDPRG[2 waltz16

PLW2 $1300000000 \mathrm{~W}$

PLW 12 0.27963999 W

PLW $13-0.22651000 \mathrm{~W}$

2 - Processing parane ters SF $\quad 100.6204380 \mathrm{MHz}$

Spectrum 128: ${ }^{13} \mathrm{C}$ NMR spectrum of $5 \mathbf{e}$ 


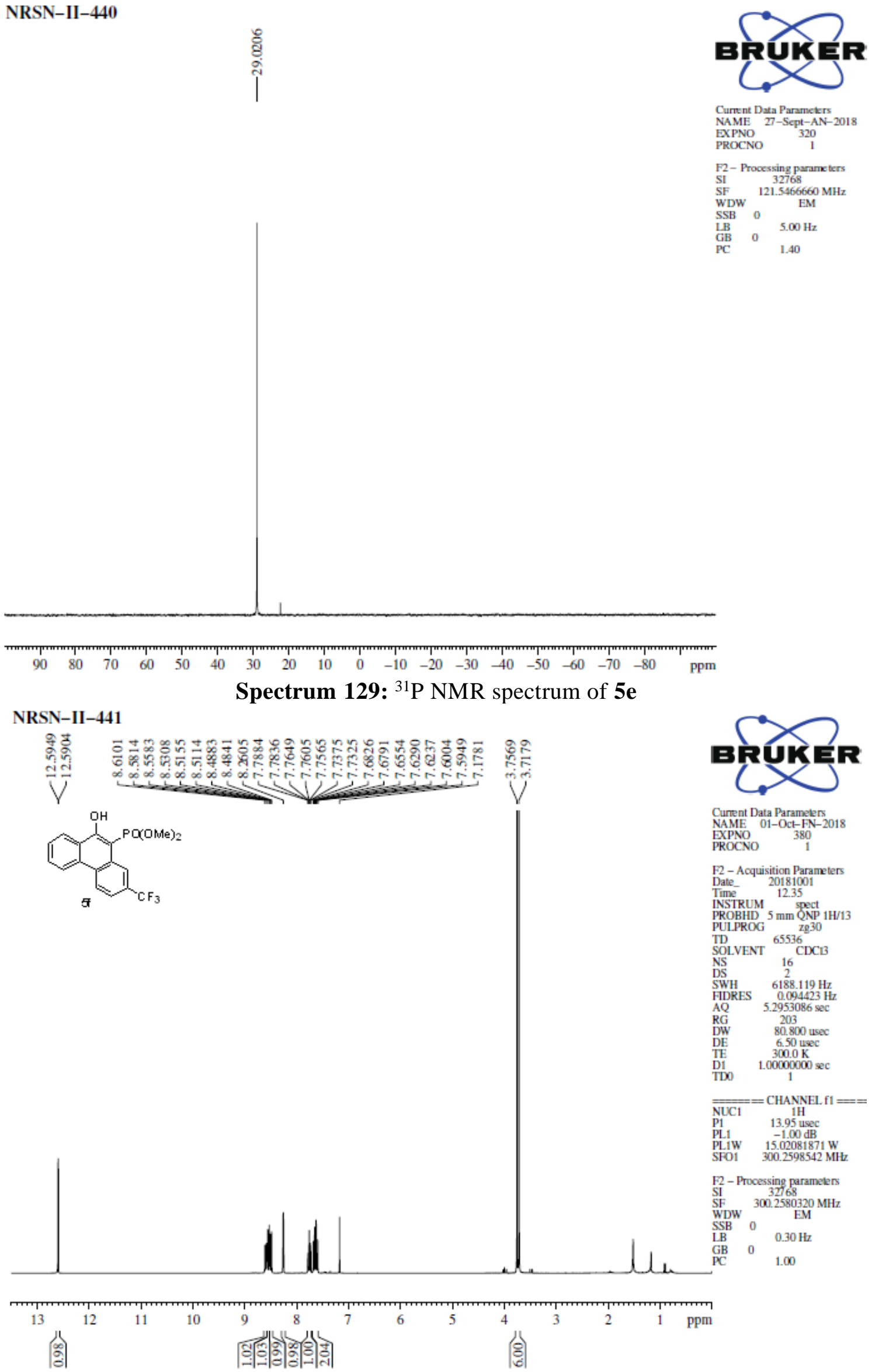

Spectrum 130: ${ }^{1} \mathrm{H}$ NMR spectrum of $\mathbf{5 f}$ 


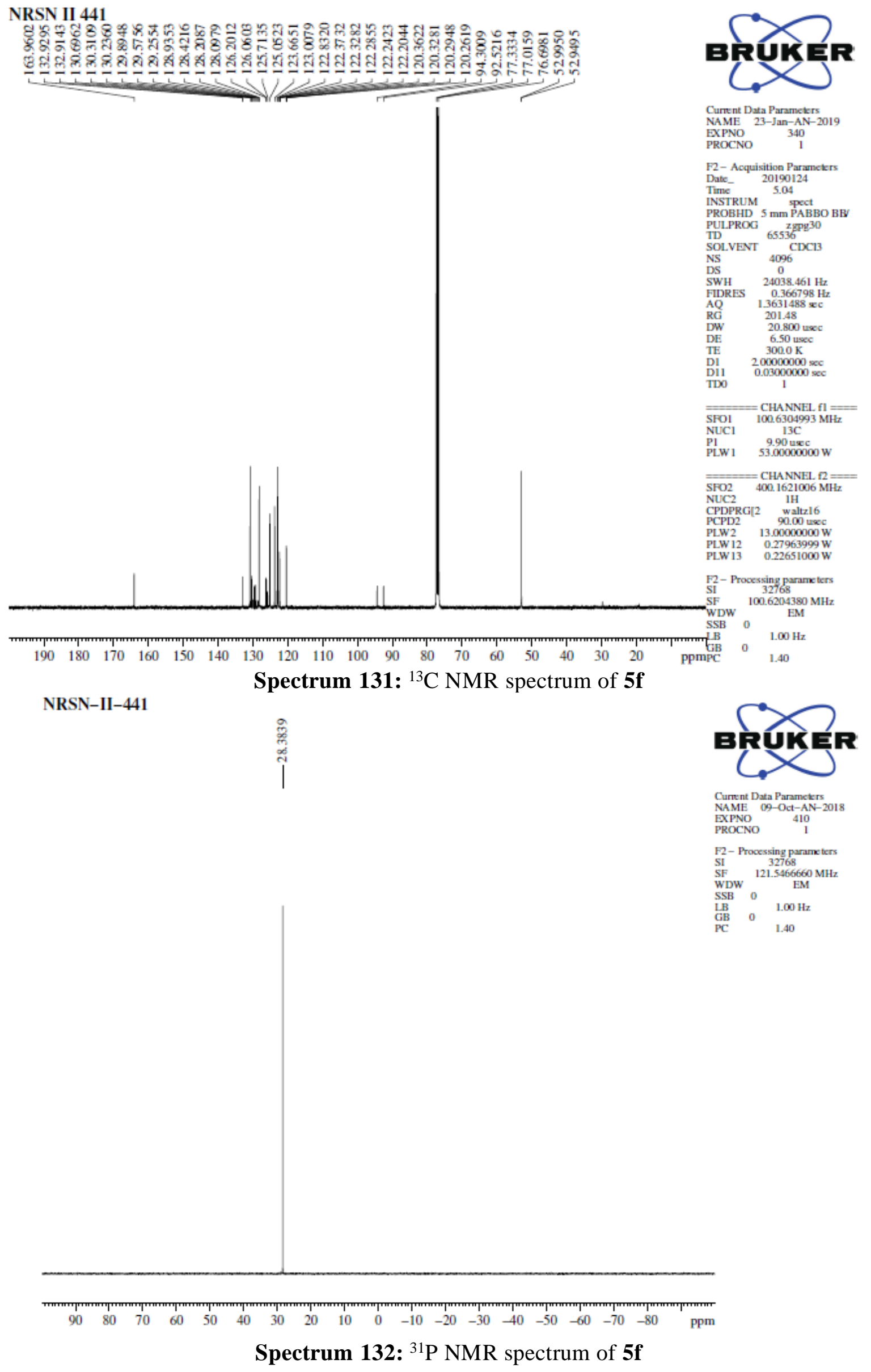




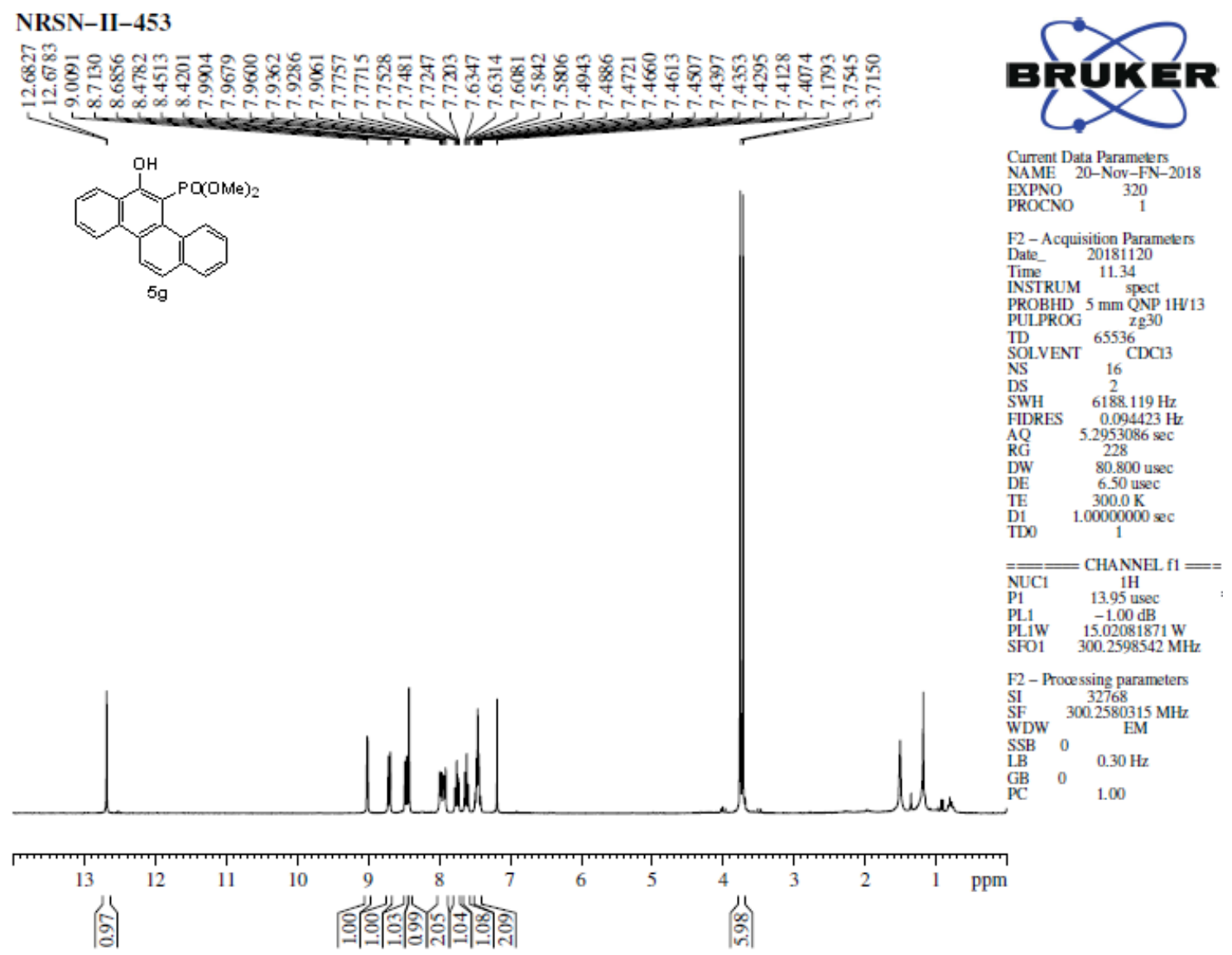

Spectrum 133: ${ }^{1} \mathrm{H}$ NMR spectrum of $5 \mathrm{~g}$

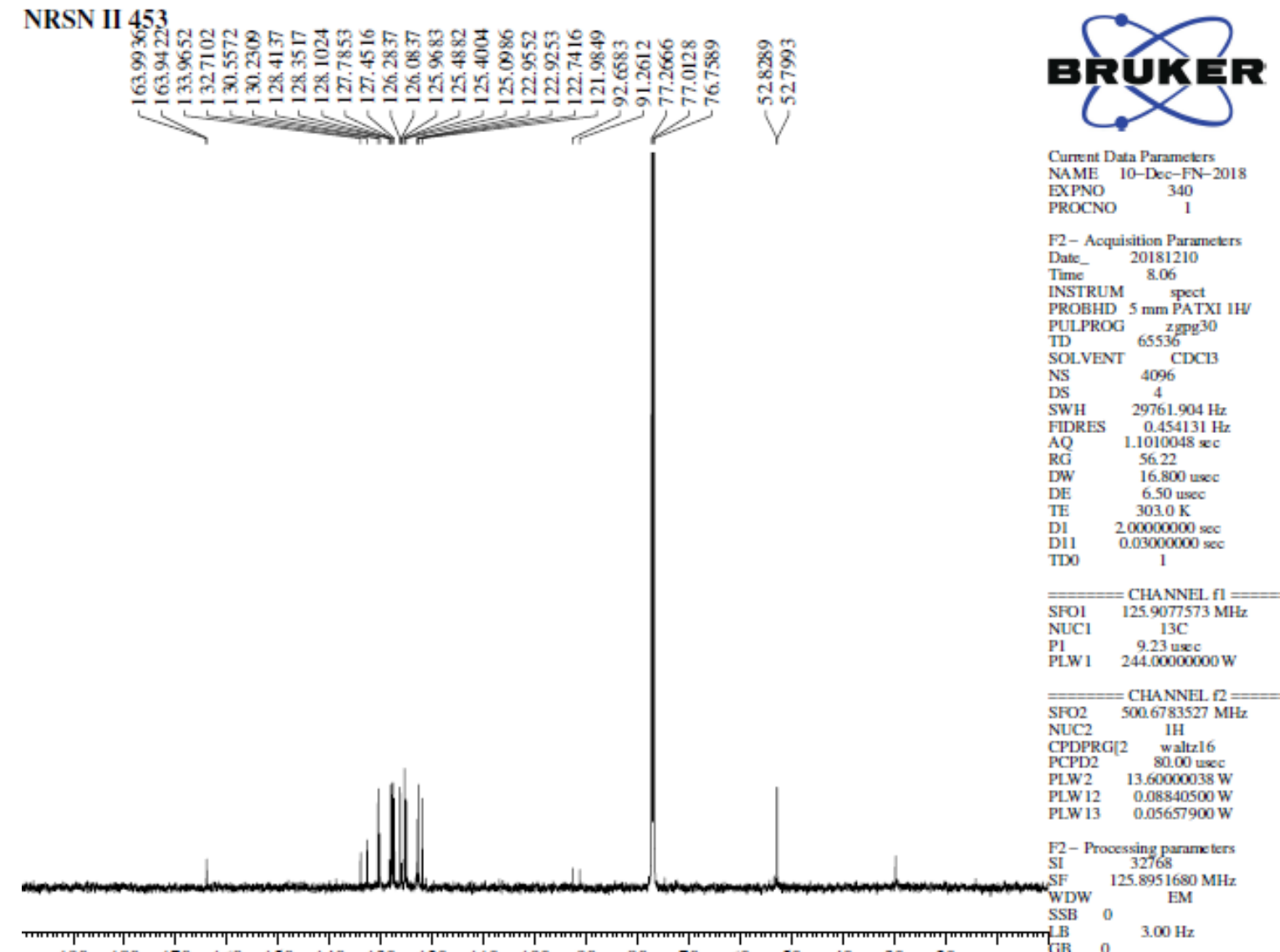

$\begin{array}{llllllllllllllllllllll}190 & 180 & 170 & 160 & 150 & 140 & 130 & 120 & 110 & 100 & 90 & 80 & 70 & 60 & 50 & 40 & 30 & 20 & \text { PpmPC } & 0 & 1.40\end{array}$

Spectrum 134: ${ }^{13} \mathrm{C}$ NMR spectrum of $5 \mathrm{~g}$ 

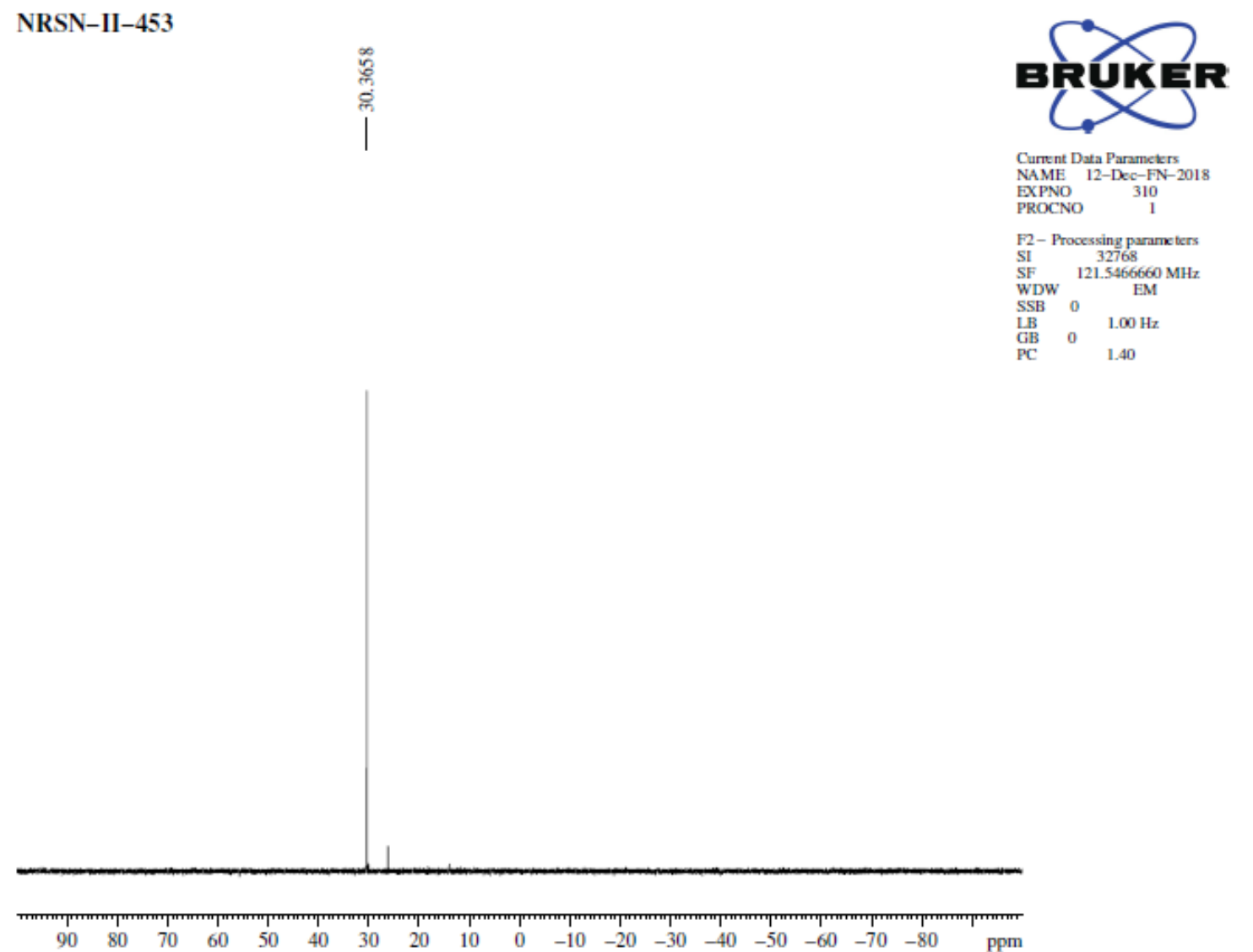

Spectrum 135: ${ }^{31} \mathrm{P}$ NMR spectrum of $\mathbf{5 g}$

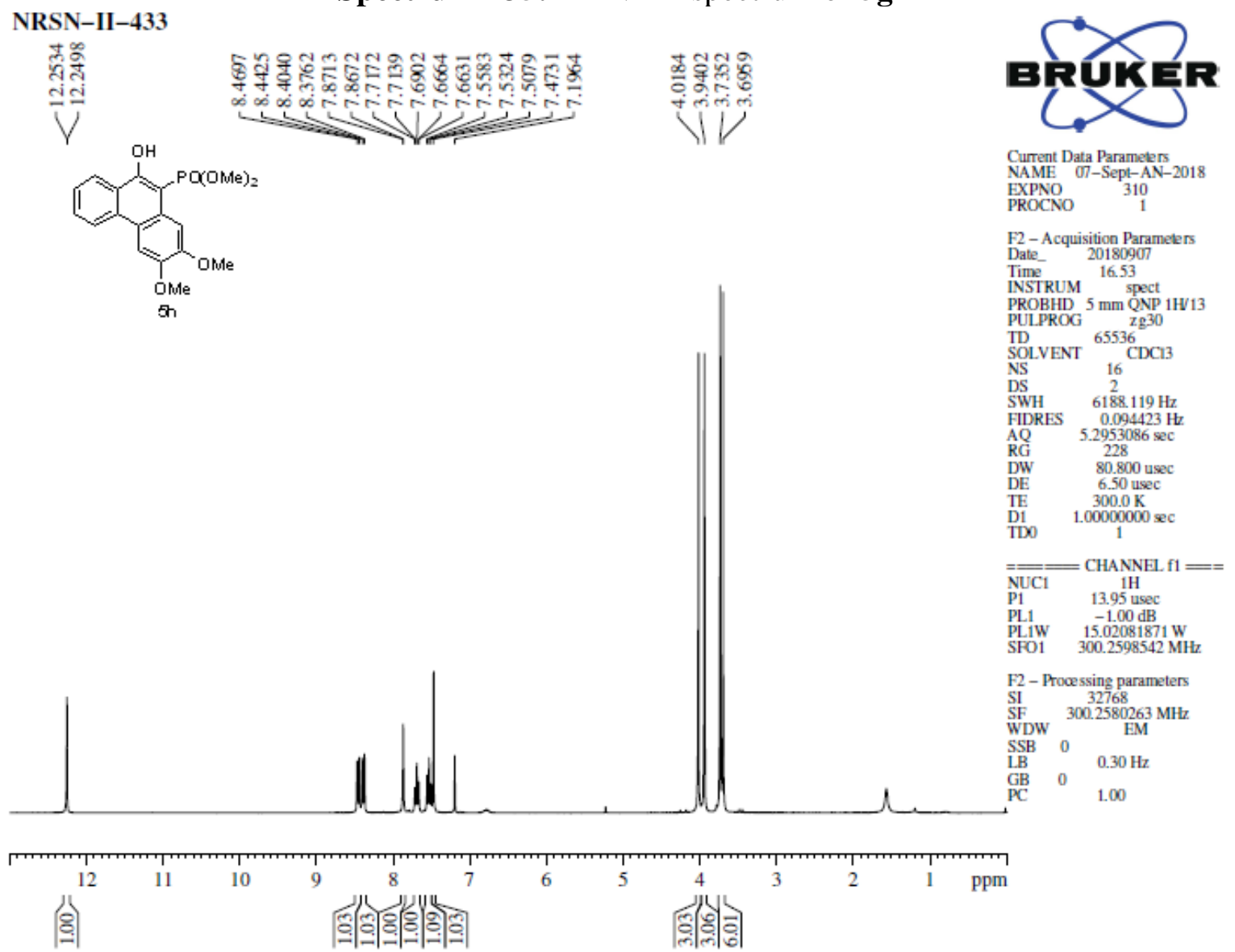

Spectrum 136: ${ }^{1} \mathrm{H}$ NMR spectrum of $\mathbf{5 h}$ 

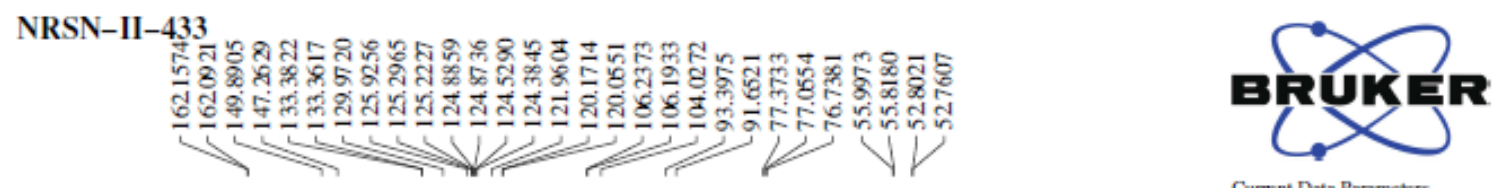

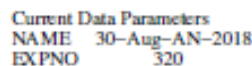
PROCNO

F2- Acquisitition Paramekrs

Date_ 20180830

Time

PROBHD 5 mPACEB PAB B

PULPROG ${ }^{2} \mathrm{zgpg}^{30}$
TD

SOLVENT CDCI

DS $\quad 512$

$\begin{array}{ll}\text { DS } & 4 \\ \text { SWH } & 24038.461 \mathrm{He}\end{array}$

FIDRES $\quad 0.366798 \mathrm{~Hz}$

AQ 1.3631488 xc

$\begin{array}{ll}\text { RG } & 201.48 \\ \text { DWW } & 20.800\end{array}$

DE 6.50 usec

TE $298.5 \mathrm{~K}$

DI $200000000 \mathrm{sec}$

$\mathrm{TD} 0 \mathrm{0}$

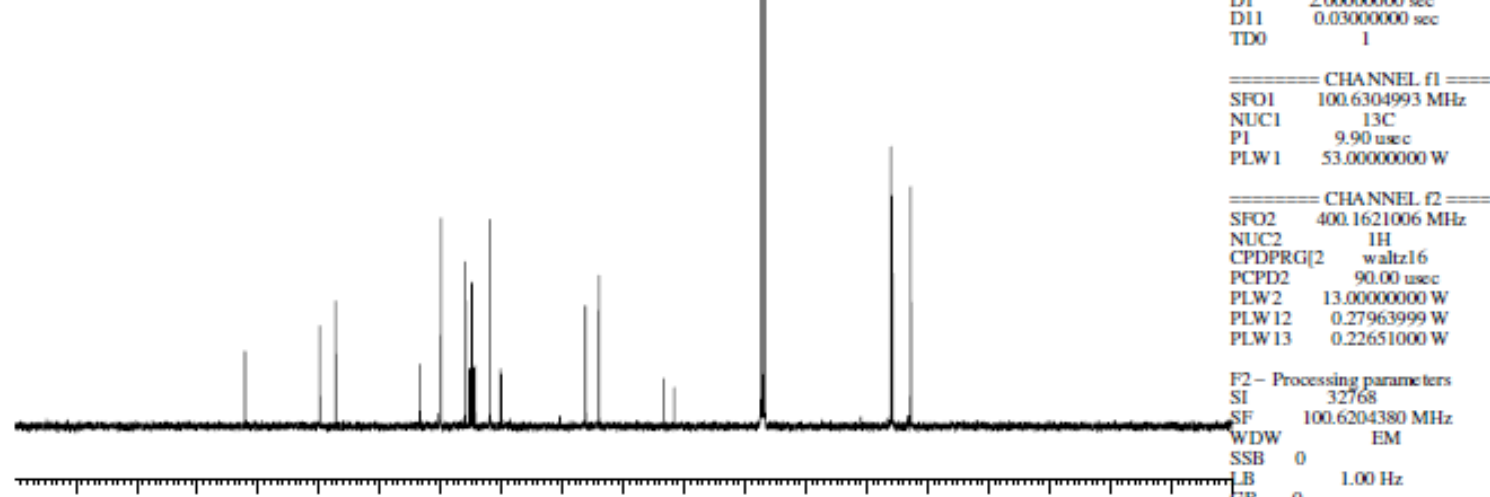

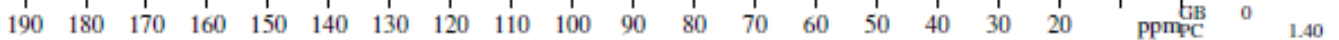

NRSN-II-433

Spectrum 137: ${ }^{13} \mathrm{C}$ NMR spectrum of $5 \mathbf{h}$

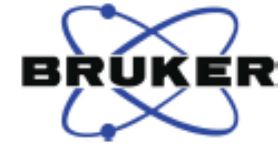

Current Data Parameters NAME 12-Sept-FYPROCNO

F2- Processing parame ters
SI 32768 WF $121.5466660 \mathrm{M}$ SSB 0 $\underset{\mathrm{GB}}{\mathrm{LB}} \quad 0.00 \mathrm{H}$

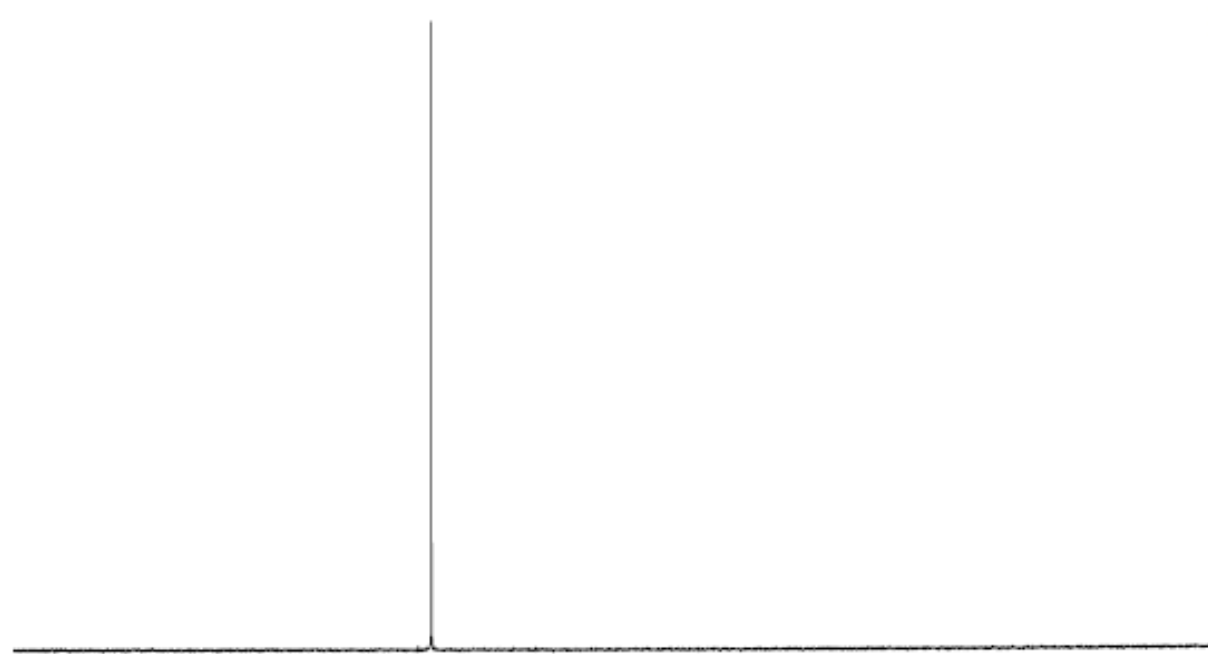

PC $\quad 1.40$

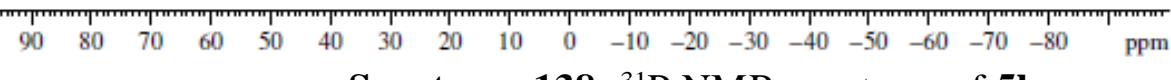

Spectrum 138: ${ }^{31} \mathrm{P}$ NMR spectrum of $\mathbf{5 h}$ 
NRSN-II-442

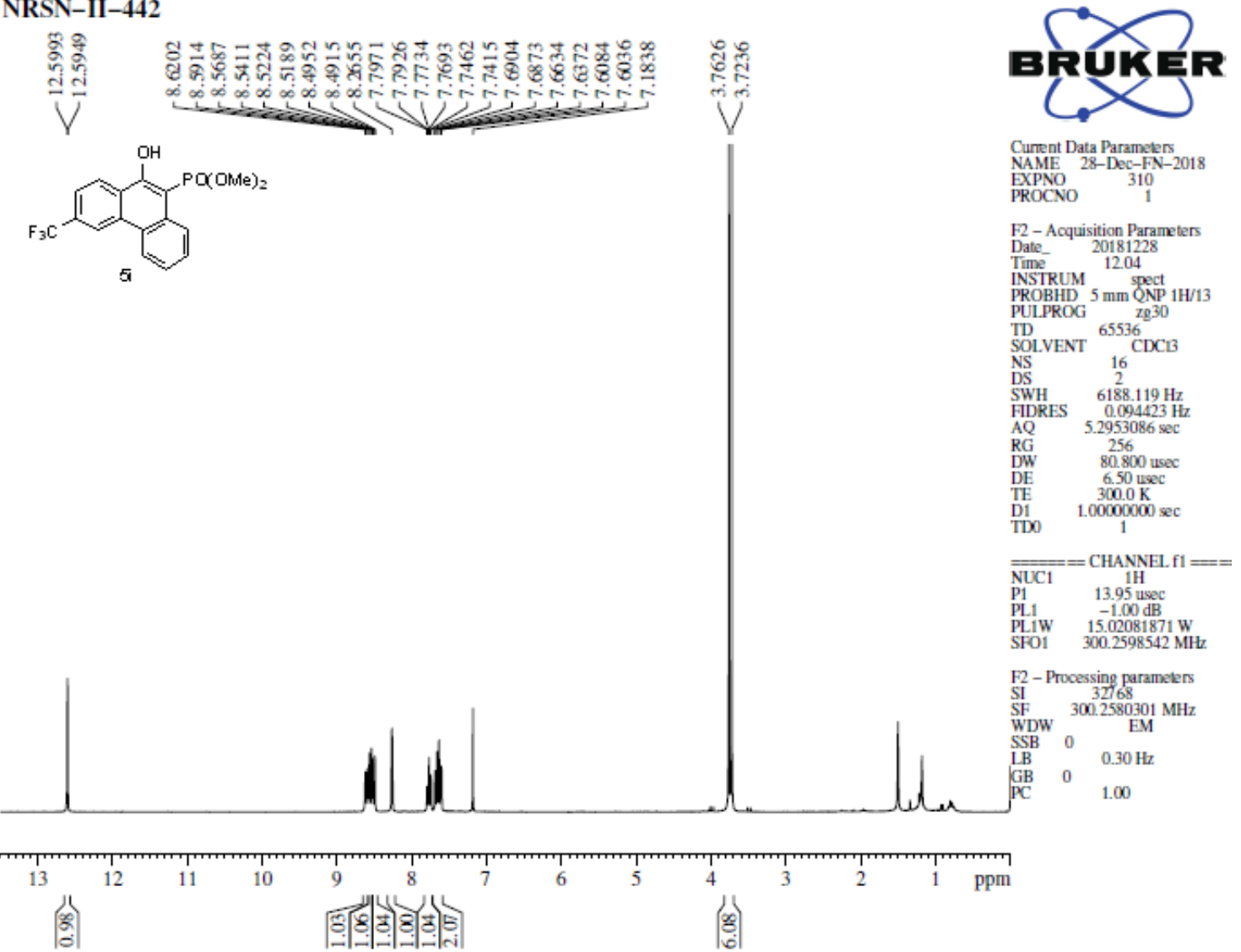

Spectrum 139: ${ }^{1} \mathrm{H}$ NMR spectrum of $\mathbf{5 i}$
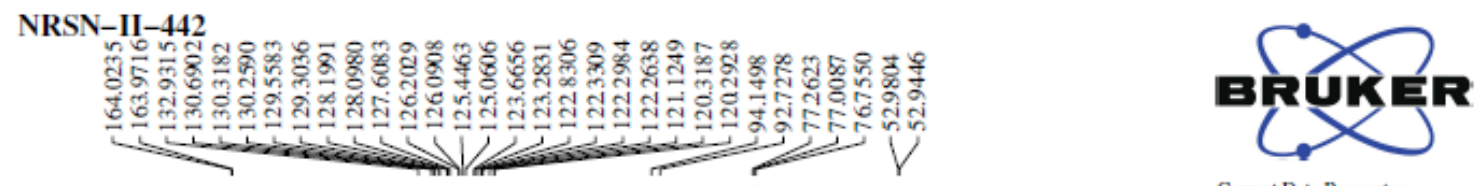

Cunent Data Parameters
NAME 18-JAN-FN-2019 $\begin{array}{lr}\text { NAME } & 18-J A N-F N-20 \\ \text { EXPNO } & 340\end{array}$ EXPNO
PROCNO

F2 - Acquisition Parameters

$\begin{array}{ll}\text { Date_ } & 20190118 \\ \text { Time } & 7.15\end{array}$

NSTRUM
spect

$\underset{\text { PULPROG }}{\text { PROBHD }} 5$ magP 30

TD ${ }_{\text {SOLVENT }}^{65536}{ }^{\text {CDC13 }}$

NS

$\begin{array}{ll}\text { DS } & 4 \\ \text { SWH } & 29761.904 \mathrm{~Hz} \\ \text { FIDRES } & 0.454131 \mathrm{~Hz}\end{array}$

FIDRES 0.454131

$\begin{array}{ll}\text { AQ } & 1.10100 \\ \text { RG } & 81.53\end{array}$

$\begin{array}{ll}\text { DW } & 16.800 \text { used } \\ \text { DE } & 6.50 \text { usec }\end{array}$

$6.50 \mathrm{usec}$
$303.0 \mathrm{~K}$

$200000000 \mathrm{sec}$

TD0 $\quad \begin{array}{ccc}0.0300000 \\ \text { TD }\end{array}$

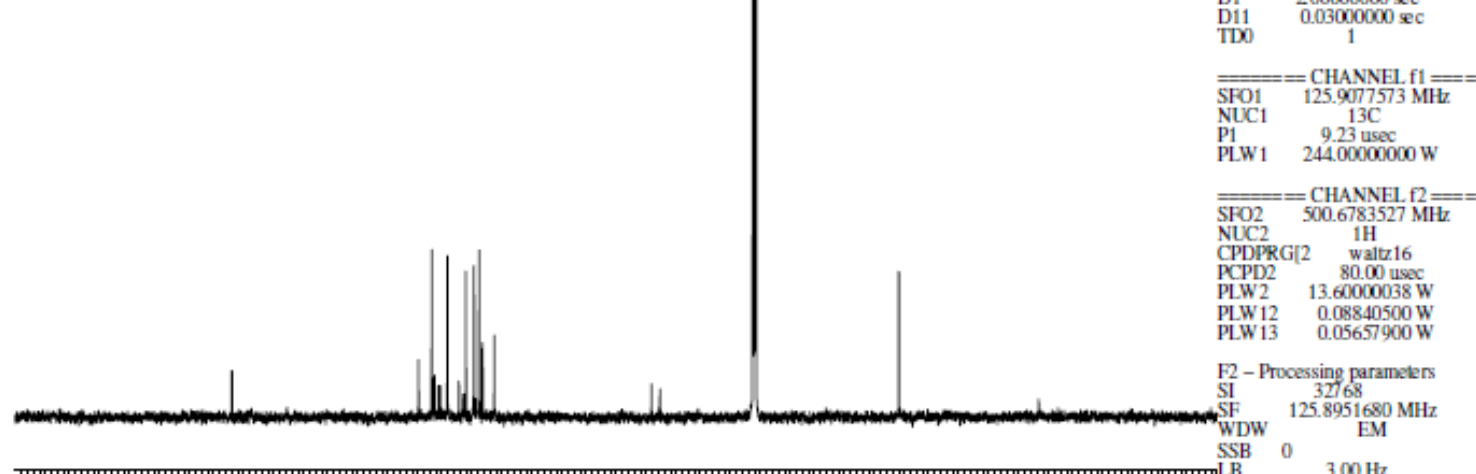

$\begin{array}{llllllllllllllllllllllll}190 & 180 & 170 & 160 & 150 & 140 & 130 & 120 & 110 & 100 & 90 & 80 & 70 & 60 & 50 & 40 & 30 & 20 & \text { ppm } & \text { PC } & 0 & 1.40\end{array}$

Spectrum 140: ${ }^{13} \mathrm{C}$ NMR spectrum of $5 \mathrm{i}$ 
NRSN-II-442
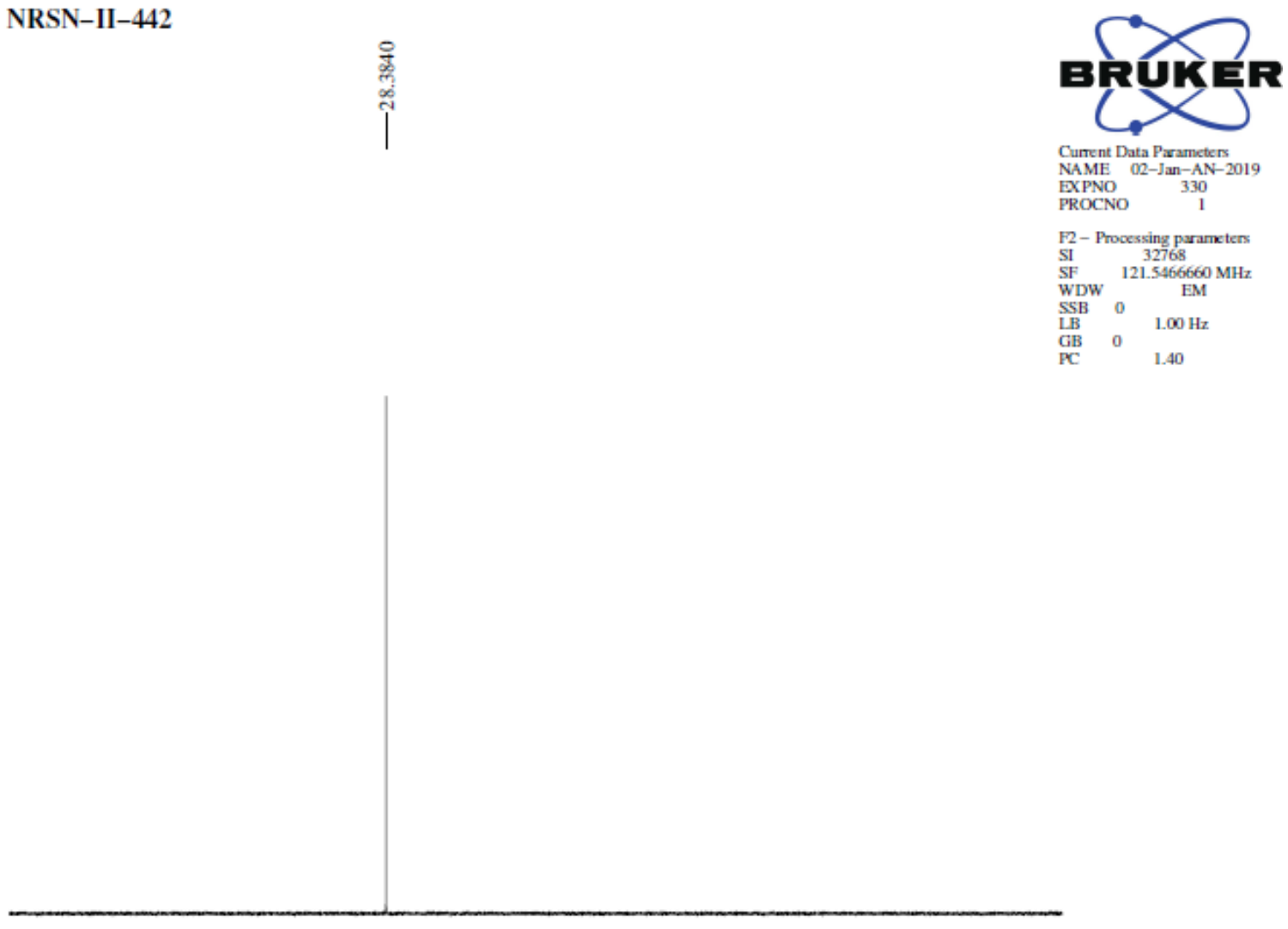

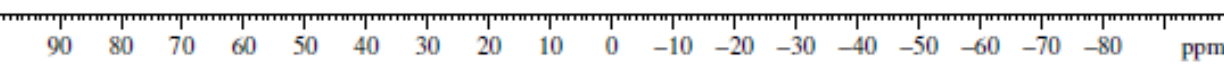
Spectrum 141: ${ }^{31} \mathrm{P}$ NMR spectrum of $5 \mathrm{i}$ 